PUB-5341

August 1992

\title{
CHEMICAL HYGIENE AND SAFETY PLAN
}
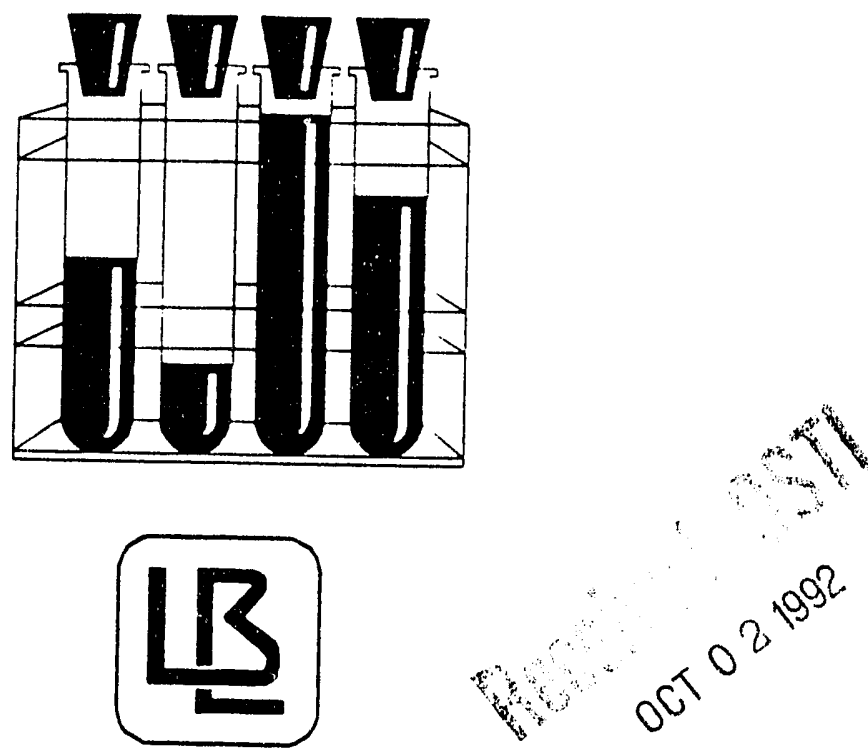

\section{Lawrence Berkeley Laboratory}

University of California

Berkeley, CA 94720 
TO: $\quad$ Distribution, PUB-3000

FROM: Klaus Berkner, Associate Laboratory Director for Operations SUBJECT: LBL Chemical Hygiene \& Safety Plan; PUB-3000 Addendum

The Department of Energy requires that LBL comply with all Federal OSHA regulations. For operations involving hazardous chemicals, both the OSHA Hazard Communication Standard (29 CFR 1910.1200) and the OSHA Laboratory Standard (29 CFR 1910.1450) apply. The Hazard Communication Standard applies to all non-laboratory operations; and, the Laboratory Standard (which is fairly new) applies to laboratory operations.

This Chemical Hygiene and Safety Plan (PUB-5341) incorporates the requirements of these OSHA standards and is designed to provide all LBL personnel with a comprehensive program to minimize the risks of working with chemicals. The Plan sets forth safety procedures and describes how LBL workers are informed about the potential chemical hazards in their work areas so they can avoid harmful exposures and safeguard their health. Many specific topics are addressed, such as procedures for working with toxins (e.g., cancercausing agents, metals, reproductive toxins), training and general responsibilities for ensuring health and safety in the laboratory.

The Plan was drafted by the EH\&S Industrial Hygiene Group, reviewed and edited by several EH\&S professionals, and further reviewed by members of the Toxic Chemicals Subcommittee, which operates under the Safety Review Committee.

The Plan provides the framework for a comprehensive chemical hygiene program. Actual implementation of the program requires detailed training and a commitment from line management. The EH\&S Industrial Hygiene and Training Groups will be working with Divisional Safety coordinators to provide division-specific workshops to help all LBL Divisions in implementing the program.

The Chemical Hygiene and Safety Plan expands upon and supersedes Chapter 5 of the LBL Health and Safety Manual (Pub-3000). Please discard chapter 5 of PUB3000 and replace it with the attached insert page that will serve to direct readers to this new publication. 


\section{CHAPTER 5 \\ CHEMICAL SAFETY}

August 28, 1992

Replace the contents of PUB-3000, Chapter 5 with this insert page.

Chapter 5, "Chemical Safety," is superseded by a new publication issued in August, 1992: PUB-5341, "Chemical Hygiene and Safety Plan". Please refer to this publication for information on chemical safety. Other chapters in PUB-3000 dealing with specific topics related to chemical safety are still in effect.

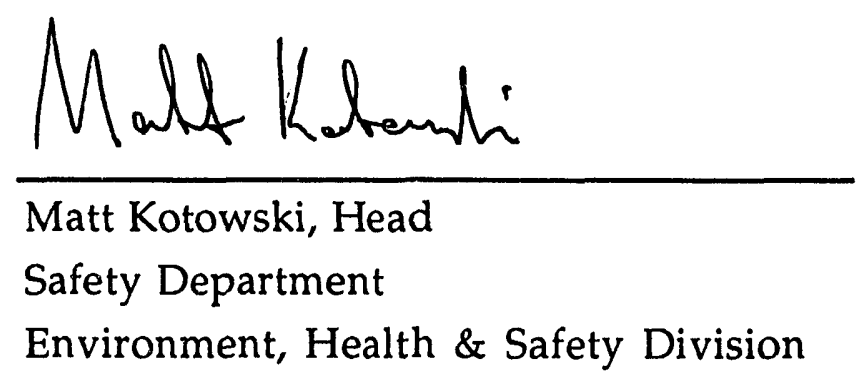


PUB-5341

August 1992

\section{CHEMICAL HYGIENE AND SAFETY PLAN}

\section{Lawrence Berkeley Laboratory \\ University of California \\ Berkeley, CA 94720}

Prepared for the U.S. Department of Energy under Contract No. DE-AC03-76SF00098

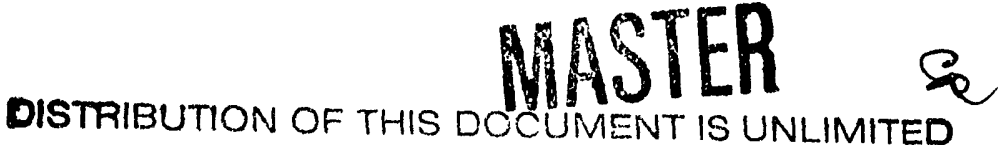




\section{Chemical Hygiene and Safety Plan}

\section{Contents}

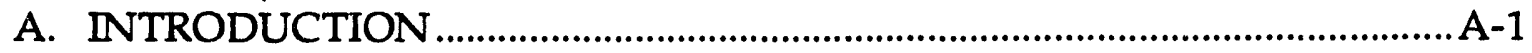

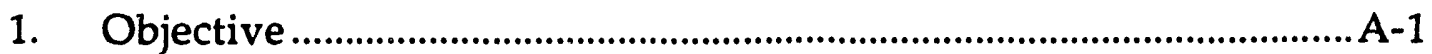

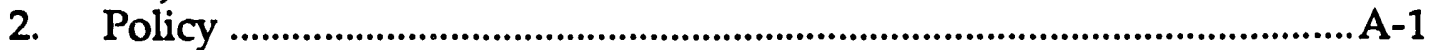

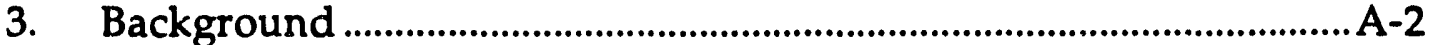

4. Scope and Implementation .....................................................................

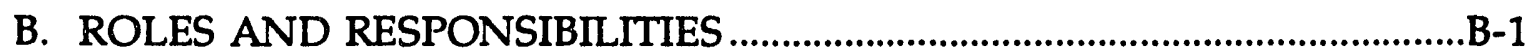

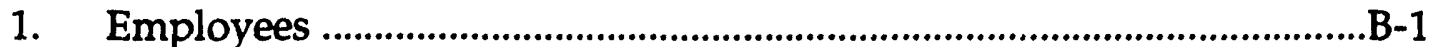

2. Principal Investigators and Supervisors ………....................................

3. Division Directors and Managers ................................................................

4 Chemical Hygiene Officer ...................................................................

5. Environment, Health \& Safety Division ................................................

6 Safety Review Committee .........................................................................

7. Purchasing.......................................................................................

8. Plant Engineering and Construction \& Maintenance.........................B-7

9. Materiel Management \& Transportation Department .......................B-8

C. HAZARD ANALYSIS AND SAFETY PROCEDURES .....................................

1. Hazardous Chemicals Defined ….........................................................

2. Hazard Assessment ......................................................................................

3. Operational Safety Procedures .............................................................4

4. Specific Safety Procedures ........................................................................

5. Variances from Safety Guidelines ......................................................6

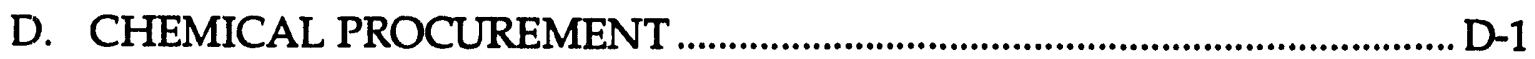

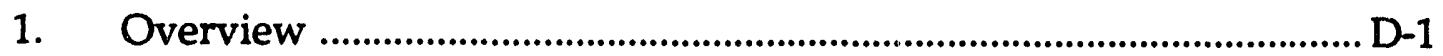

2. Purchase-Controlled Substances ................................................. D-2

3. Industrial Hygiene Pre-Approval/Review........................................ D-2

E. STANDARD HYGIENE AND SAFETY PRACTICES ….................................E-1

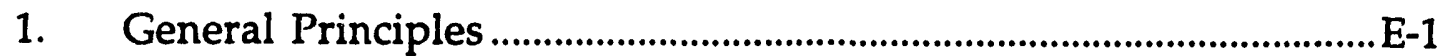

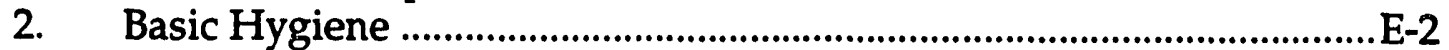

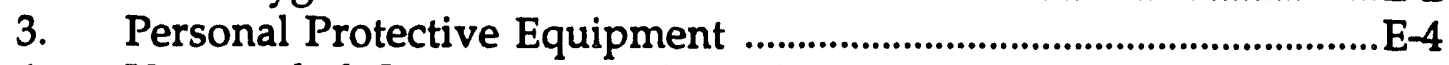

4. Unattended Operations and Working Alone ......................................E-6

5. Housekeeping .........................................................................................

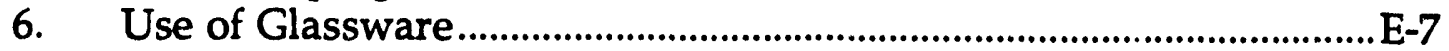


E. STANDARD HYGIENE AND SAFETY PRACTICES, Continued

7. Transport of Hazardous Materials...................................................... E-8

8. Labeling ……........................................................................................

9. Storage of Chemicals ….....................................................................

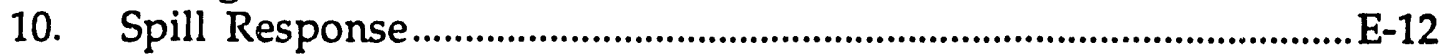

11. Disposal of Chemicals ...............................................................................

F. HAZARD CONTROL MEASURES ……....................................................

1. Criteria for Control Measures.............................................................-1

2. Engineering Controls ........................................................................

General Ventilation .........................................................................

Local Exhaust Ventilation (Hoods) ................................................ F-5

Biosafety Cabinets (Laminar Flow Hoods) .....................................F-8

Additional Ventilation Considerations .......................................F-9

3. Administrative Controls ..................................................................F-10

Correct Work Practices .................................................................10

Use of Restricted Areas................................................................F-11

4. Personal Protective Equipment ...................................................

5. Safety and Emergency Equipment ....................................................13

6. Preventive Maintenance ..................................................................16

7. Equipment Decommissioning ..............................................................F-18

G. USING HIGH HAZARD CHEMICALS......................................................-1

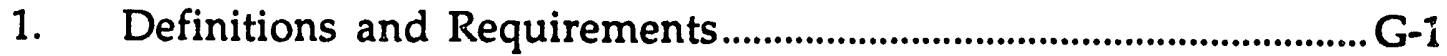

2. Chemical Carcinogens .......................................................................

Carcinogen Classification............................................................ G-4

Select Carcinogens ............................................................................... G-5

Program Basics …........................................................................

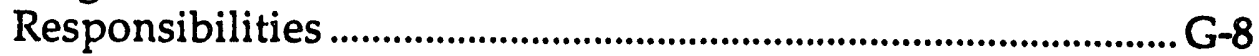

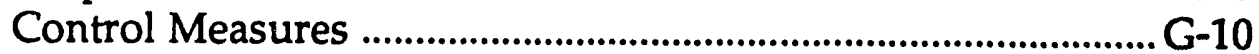

Exposure Monitoring and Medical Services............................... G-13

3. Reproductive Toxins .....................................................................14

4. Toxic and Pyrophoric Gases ….....................................................16

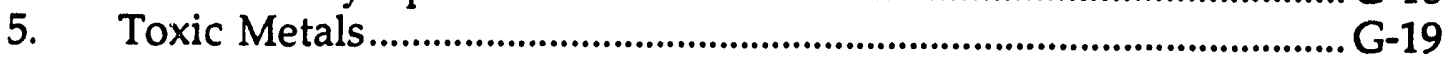

6. Reactive Chemicals.........................................................................24

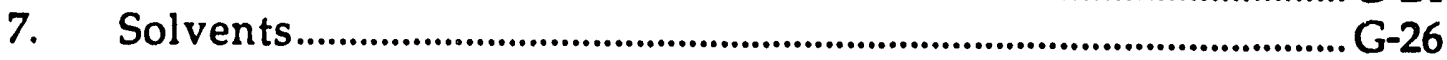

Flammable and Combustible Liquids ..........................................26

Chlorinated Hydrocarbons ............................................................31

Fluorocarbon Solvents ............................................................. G-35

8. Polychlorinated Biphenyls ..............................................................36

9. Physical Hazards ......................................................................................... G-37 
1. Requirements $\mathrm{H}-1$

2. Hazard Communication Resources ....................................................

3. Training Resources .....................................................................

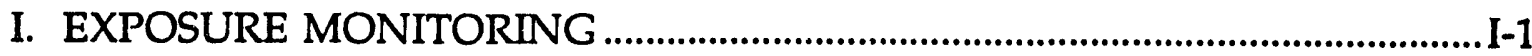

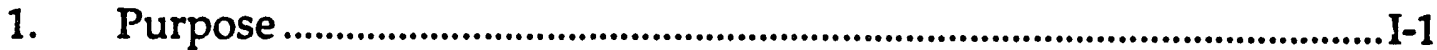

2. Hazard Assessment and Criteria for Monitoring................................I-2

3. Substances that Require Monitoring .......................................................-3

4. Interpretation of Results .........................................................................

J. MEDICAL CONSULTATIONS AND SURVEILI.ANCE ................................... -

1. Overview of Medical Services ................................................................... -1

2. Medical Surveillance for Exposure to Hazardous Chemicals............. J-2

3. Identifying the Need for Medical Surveillance ....................................... J-3

4. When OSHA Regulations Require Medical Surveillance...................J-5

5. Medical Consultations in Response to Symptoms and/or Chemical Releases .....................................................................J-7

6. Exposures to Carcinogens .......................................................................... J-8

7. Exposures to Reproductive Toxins ................................................................. J-9

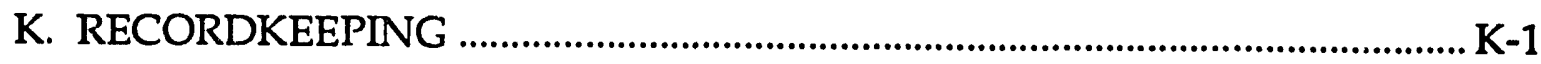

L. REFERENCES AND INFORMATION RESOURCES ..................................... L-1 


\section{Chemical Hygiene and Safety Plan}

\section{Appendices}

Appendix 1. OSHA Hazard Communication Standard (29 CFR 1910.1200)

Appendix 2. OSHA Laboratory Standard (29 CFR 1910.1450)

Appendix 3. Glossary of Terms

Appendix 4. The Facility Notebook and Specific Safety Procedures (SSPs)

Appendix 5. University of California Purchase Requisition

Appendix 6. Glove Selection Guides

Appendix 7. Labeling Information

Appendix 8. Chemical Storage Guidelines

Appendix 9. Select Carcinogens

Appendix 10. ACGIH Carcinogens

Appendix 11. Composite Working Select Carcinogen List

Appendix 12. Carcinogen Exposure Assessment Survey Form

\& Industrial Hygiene Hazard Evaluation Form

Appendix 13. Reproductive Toxins

Appendix 14. Safety Training Check List

Appendix 15. Chemical Information (MSDSs, Ethernet, and TOMES)

Appendix 16. Subject Index 


\section{A. INTRODUCTION}

\section{Objective}

The objective of this Chemical Hygiene and Safety Plan (CHSP) is to provide specific guidance to all LBL employees and contractors who use hazardous chemicals. This Plan, when implemented, fulfills the requirements of both the Federal OSHA Laboratory Standard (29 CFR 1910.1450) for laboratory workers, and the Federal OSHA Hazard Communication Standard (29 CFR 1910.1200) for non-laboratory operations (e.g., shops). It sets forth safety procedures and describes how LBL employees are informed about the potential chemical hazards in their work areas so they can avoid harmful exposures and safeguard their health. Generally, communication of this Plan will occur through training and the Plan will serve as a the framework and reference guide for that training.

Health and safety hazards at Lawrence Berkeley Laboratory are as varied as the operations and procedures performed at LBL. They range from common industrial hazards presented by shop machinery and motor vehicles to the special hazards associated with research, including those presented by toxic chemicals, chemical carcinogens, heavy metals, and flammaijle liquids. Physical hazards range from high voltage electricity and pressure vessels to ionizing radiation.

This Plan focuses on chemical hazards. Chemical hazards are present when materials are reactive, explosive, corrosive, flammable, or toxic, and more than one hazardous property may be presented by a single material. Many chemicals are relatively nonhazardous by themselves but become dangerous when they interact with other substances, either in planned experiments or by accidental contact.

\section{Policy}

It is LBL's policy to provide a safe and healthful working environment for its employees. It is also LBL's policy to protect property from damage or loss caused by an accident and to prevent any harm to the general public or the environment as a result of activities at LBL. Every supervisor is responsible for managing the safety of the people he or she supervises. It is a requirement of employment and a 
precondition for using Laboratory facilities that every employee, guest, visiting scientist, or contractor working on or off site, must be familiar with and implement LBL safety standards. Individual Divisions and facilities may have additional rules and procedures that must also be followed.

This Chemical Hygiene and Safety Plan is intended to ensure that all LBL workers are minimally exposed to hazardous chemicals to the extent reasonably possible. Exposures to airborne chemicals are to be kept below the Permissible Exposure Levels set forth in the Code of Federal Regulations (29 CFR 1910.1000) and the Threshold Limit Values set forth by the American Conference of Governmental Industrial Hygienists. This is accomplished with the proper combination of engineering, administrative, and personal protective controls.

\section{Background}

The Occupational Safety and Health Administration (OSHA) first promulgated the Toxic and Hazardous Substances Hazard Communication Standard (29 CFR 1910.1200) in 1983 for manufacturing industries. The standard was developed to inform workers who worked with hazardous chemicals of the risks associated with those chemicals. Hazard information was to be transmitted by material safety data sheets (MSDSs) and container labels. In 1987, the Hazard Communication Standard (included as Appendix 1) was expanded to cover non-manufacturing as well as manufacturing industries. The Hazard Communication Standard also applied to laboratories, but with less requirements, because a separate Laboratory Standard was to specifically address laboratories.

The "Laboratory Standard," published as "Occupational Exposures to Hazardous Materials in Laboratories" (included as Appendix 2), was promulgated in 1990. OSHA promulgated the Laboratory Standard on the basis that (1) laboratories typically differ from industrial operations in their use and handling of hazardous chemicals, and (2) a different approach than that found in OSHA's substance-specific health standards is warranted to protect laboratory workerj. 
"Laboratory use" is defined as "work with substances in which all of the following conditions are met":

- Chemical manipulations are carried out on a laboratory scale. That is, the work with chemicals is in containers of a size that could be easily and safely manipulated by one person.

- Multiple chemical procedures are used.

- Protective laboratory practices and equipment are available and commonly used.

- The procedures involved are not part of a production process whose function is to produce commercial quantities of materials, nor do the procedures in any way simulate a production process.

A glossary of terms common to both Standards, which includes the above definition, is included as Appendix 3.

The Laboratory Standard was written to supersede the Hazard Communication Standard and substance-specific OSHA health standards for laboratory operations. The Laboratory Standard differs from many OSHA health standards in that it does not establish new exposure limits, but requires additional performance provisions designed to protect laboratory workers from potential hazards in their work environments. The manner in which this is achieved is left to the discretion of each laboratory, but must involve the formulation and implementation of a Chemical Hygiene Plan.

The Department of Energy has mandated that LBL comply with Federal OSHA regulations. Consequently, both the Hazard Communication Standard and the Laboratory Standard apply, depending on the nature of the operation (i.e., laboratory operations vs. non-laboratory operations). The following section explains the scope of this Chemical Hygiene and Safety Plan. 


\section{Scope and Implementation}

This Chemical Hygiene and Safety Plan (CHSP) is designed to provide all LBL personnel with a program to minimize the risks of working with chemicals; therefore, it applies to all operations where hazardous chemicals are used. LBL operatiuns that include the use of hazardous chemicals are both laboratory and nonlaboratory operations. Generally, the non-laboratory operations are shops (e.g., light assembly, etc.); however, some office-type operations might include the infrequent use of chemicals.

The advantage of having one broad plan for laboratory and non-laboratory operations is that it sets forth a standardized framework for chemical hygiene practices, information dissemination, and training at LBL. And, uniformity and simplicity help promote and ensure regulatory compliance.

The CHSP expands upon and supersedes Chapter 5 of the LBL Health and Safety Manual (Pub-3000). However, other chapters of the LBL Health and Safety Manual also contain pertinent chemical hygiene information, and these chapters are referenced as appropriate.

Note that while this CHSP provides the framework for chemical hygiene procedures, the Laboratory Standard is not fully implemented until facility-specific chemical hygiene information is provided. Facility-specific hazard information and written safety procedures are to be provided by each LBL facility within a notebook, the "Facility Notebook." The overall purpose of the Facility Notebook is to provide an organized body of facility-specific information and documentation to support compliance with DOE Order 5480.19, "Conduct of Operations," and to provide facility-specific health and safety information required by the Laboratory Standard and other OSHA standards.

The Facility Notebook serves to provide the following site-specific information required by the Laboratory Standard:

- Facility description and chemical hazard identification;

- Operator responsibilities; 
- Lab/Shop-Specific Safety Procedures (SSPs) for routine operations using hazardous chemicals;

- Operational Safety Procedures (OSPs) for all extremely hazardous operations (those utilizing toxic gases, high radiation, etc.);

- Chemical hazard information;

- Recordkeeping; and

- Emergency protocols. 


\section{B. ROLES AND RESPONSIBILITIES}

Every employee, visiting scientist, student, or other person performing work at the LBL or at one of the LBL's off-site locations is responsible for understanding the properties of the chemicals with which they will work, following all applicable safety standards and for taking the initiative in consulting with resource groups (e.g., individual EH\&S Departments) when safety-related assistance or advice is needed. When faced with an unexpected threat of malfunction, injury, or damage, employees are expected to choose a course of action that provides the most protection to themselves and to others in the area. Every employee is expected to report to the supervisor any unsafe condition or practice seen in the area that would not permit him/her to work safely.

The following sections further define the roles and responsibilities of various parties.

\section{Employees}

Employees at LBL must:

- Work safely by observing safety standards, guidelines, and procedures, and by using good judgement based on training and expertise.

- Report unsafe conditions or injuries to the Principal Investigator or Supervisor immediately.

- Stop work under unsafe conditions.

- Be familiar with and follow emergency procedures.

- Attend safety training meetings.

- When ordering materials, identify hazardous chemicals in the hazard review section of the LBL purchase requisition form. 
LBL employees have the right to:

- Be notified of measured or suspected exposures to harmful substances above legal occupational exposure limits.

- Be given an opportunity to observe monitoring for hazardous substances.

- Request a medical consultation.

- Access their workplace medical and exposure records.

- Refuse to work in unsafe conditions or to perform work that could create a hazard to them or other workers.

- File confidential health and safety complaints with the local Department of Energy (DOE) office. Employees may write a letter, submit Form 5480.4 (available from EH\&S), or phone in a complaint to the Environmental Safety \& Support Department, DOE-SAN, 1333 Broadway, Suite 650, Oakland, CA 94612; (510) 273-7963. Complaints will be investigated promptly, and all information is strictly confidential.

\section{Principal Investigators and Supervisors}

The Principal Investigator or Laboratory/Shop Supervisor:

- Has responsibility for day-to-day laboratory or shop personnel and operations.

- Enforces LBL safety rules and establishes specific safety procedures (SSPs) for all pertinent laboratory/shop operations and procedures (see Appendix 4).

- Ensures that employees, students, visiting scientists, and participating guests are trained in safety procedures, are familiar with the specific hazards in their work area(s), and have knowledge of the specific warning signs and symptoms of being exposed to the chemicals with which they work. 
- Corrects improper work practices, identifies deficient conditions that could result in personal injury, and develops a positive attitude among employees toward hazard and accident prevention.

- Investigates and reports to Environment, Health \& Safety and to their Department Head or Director every accident (requiring more than first aid) and significant "near miss" accidents that might have resulted in serious injury. [Note: Some accidents are reportable to DOE as an Unusual Occurrence Report (UOR). Contact the EH\&S UOR Coordinator at Ext. 7612.]

- Ensures that a corrective action identified from an accident investigation or laboratory/shop inspection is implemented.

- Ensures hazards are properly labeled and controlled.

- Obtains and maintains copies of Material Safety Data Sheets (MSDSs), as required for each hazardous material used in the work area and ensures that they are readily accessible to all employees.

- Ensures that the CHSP, Pub-3000, and the Facility Notebook (with specific safety procedures) are readily available to all employees.

- Identifies hazardous materials on the LBL Purchase Requisition Form when ordering.

- Conducts periodic inspections or self-assessments to audit compliance with health and safety procedures.

- Conducts a review of laboratory/shop hazards, controls, procedures at least annually and when any new or altered procedure is introduced.

- Conducts an inventory of their hazardous materials at least annually, and more often if substantial changes occur in the quantities and/or types of materials. 


\section{Division Directors and Managers}

Division Directors and Managers must:

- Ensure that environmental, health, and safety concerns reported by their employees are addressed.

- Provide the Supervisors and Principal Investigators with the resources needed to safely manage their staff and operations.

- Ensure that life-safety hazards are properly controlled in the facilities and operations for which they have responsibility.

- Ensure that a mechanism is in place to review and evaluate new research projects or operations and other unique activities for their potential hazards and for adequacy of the planned safety controls.

- Determine whether a proposed research project or shop operation requires an Operational Safety Procedure (OSP) during the project proposal phase.

- Ensure that accidents are thoroughly investigated and corrective action is taken to prevent a reoccurrence.

- Ensure documentation of key safety and health decisions in the minutes of the Division Safety committee.

- Ensure that all violations of codes and safety standards identified by reviews or inspections are corrected in a timely manner.

\section{Chemical Hygiene Officer}

The LBL "Chemical Hygiene Officer" is the EH\&S Industrial Hygiene Group. The staff industrial hygienists and Group Leader all serve as Chemical Hygiene Officers. Additionally, an LBL Division Director may assign a safety coordinator to act as a chemical hygiene officer for the whole division or select departments within the division. The Industrial Hygiene Group will support the efforts of all division 
hygiene officers. The Group provides the following industrial hygiene expertise and services:

- Consults with the Toxic Chemicals Subcommittee on the development and implementation of chemical hygiene and safety policies and practices.

- Assists Principal Investigators and laboratory/shop supervisors in developing safe laboratory or shop operations and procedures prior to start-up.

- Keeps current concerning the occupational and environmental legal requirements respecting hazardous substances in the laboratory and shop environments and informs management.

- Reviews the procurement and use of high hazard chemicals in the laboratories and shops, and on a case-by-case basis, determines the adequacy of safety procedures and the apparent levels of training/knowledge of the persons conducting those procedures.

- Reviews the CHSP at least anr.ually and revises it as necessary.

- Arranges site inspections to review Specific Safety Procedures (SSPs), audits compliance, and provides chemical hygiene support services, which includes exposure monitoring and medical referrals.

\section{Environment, Health \& Safety Division}

The Environment, Health \& Safety (EH\&S) Division assists supervisors and employees in working safely by providing information on the hazardous properties of materials, recommending (and at times, requiring) methods for controlling the hazards of specific operations, and by monitoring the work environment. The Industrial Hygiene Group in particular has a strong role in chemical hygiene and safety, serving as a team of Chemical Hygiene Officers. With regard to hazard communication requirements, the EH\&S Division: 
- Assists the Toxic Chemicals Subcommittee by providing them with technical and regulatory health and safety information.

- Maintains a central file of Material Safety Data Sheets (MSDSs).

- Provides generic training programs.

- Assists supervisors in developing hazard-specific training programs.

The Fire Department, a department within the EH\&S Division, is responsible for protecting people and property from fires, explosions and other hazards through prevention and expeditious control of such events. The Fire Department provides first-response resource and transportation services in medical emergencies. In addition, the Department reviews and audits fire safety concerns related to flammable gases and liquids.

For further review of these responsibilities, refer to the LBL Health and Safety Manual (Pub-3000), Chapter 12. Specific responsibilities for the EH\&S Division are provided in Chapter 2 of Pub-3000.

\section{Safety Review Committee}

The Safety Review Committee (SRC):

- Reports to and is advisory to the Associate Laboratory Director of Operations on matters of health and safety.

- Acts as a resource to the Division Director of EH\&S for reviewing and recommending methods and/or policies for addressing special safety problems.

- Directs several subcommittees that address special safety issues of the Laboratory's programs, such as the Toxic Chemicals Subcommittee. The Toxic Chemicals Subcommittee helps in reviewing the Chemical Hygiene and Safety Plan on an annual basis. The Subcommittee meets at least quarterly and publishes meeting minutes. 


\section{Purchasing}

The Purchasing Department:

- Ensures that chemical purchases have been approved by the Principal Investigator.

- Coordinates specialized distribution procedures for high hazard materials.

- Assists Principal Investigators and Supervisors in the procuring Material Safety Data Sheets (MSDS).

- Submits copies of all requests for hazardous materials to the Industrial Hygiene Group.

- Identifies controlled items (e.g., toxic gases) that need special hazard review.

\section{Plant Engineering and Construction \& Maintenance Departments}

The Plant Engineering Department together with the Construction \& Maintenance Department:

- Work with EH\&S to ensure that existing and new laboratories/shops comply with applicable Life Safety and related building codes.

- Assist the Principal Investigator or Laboratory/Shop Supervisor in the installation of effective engineering controls.

- Maintain a proactive Preventative Maintenance Program to ensure that laboratory controls and emergency equipment are in proper operating condition.

- Alert on-site construction/equipment contractors to hazardous materials in their immediate work areas. 
- Alerts on-site construction/equipment contractors that they must provide to their employees information on hazardous materials that they bring to the worksite.

- Requests MSDSs from contractors for materials that they bring on site.

\section{Materiel Management and Transportation Department}

The Materiel Management and Transportation Department:

- Ensures the proper storage and handling of chemicals once delivered to LBL, prior to their disbursement.

- Transports chemicals safely.

- Notifies EH\&S when items needing special hazard review are received. 


\section{HAZARD ANALYSIS AND SAFETY PROCEDURES}

Every operation or procedure that involves the use of hazardous materials must have written health and safety guidelines or procedures that analyze the hazards and provide the following information:

(1) Responsible parties

(2) A description of the operation or procedure (e.g., protein extraction, painting, electroplating)

(3) Identification of the hazardous materials utilized in the operation/procedure

(4) A hazard analysis describing all potential hazards inherent in the use, storage, and manipulation of these substances

(5) The intended control measures (engineering, administrative, and personal protective equipment)

(6) Provisions for waste handling, spill clean-up, decontamination, etc.

(7) Reference to pertinent medical surveillance protocols

For most operations and procedures, a simple two-page form (see Appendix 4) will suffice to generate a "Lab/Shop Specific Safety Procedure" (SSP). For very hazardous operations and procedures, a more detailed Operational Safety Procedure (OSP) is necessary. Both procedures are to be kept with or in the Facility Notebook. The two basic types of written safety procedures are described in greater detail below (Sections C.2 and C.3) following discussions that define hazardous chemicals and hazard assessment (Sections C.1 and C.2).

\section{Hazardous Chemicals Defined}

The term "hazardous chemical" is defined below for the purpose of demonstrating when the Chemical Hygiene and Safety Plan (CHSP) is to implemented. Thus, if 
any chemical meeting the definition of "hazardous chemical" as it relates to health hazards is used by a laboratory or shop, the CHSP is to be implemented for that laboratory or shop.

A hazardous chemical is a chemical that can potentially pose a health hazard and/or a physical hazard. The term "health hazard" includes chemicals that are carcinogens, toxic or highly toxic agents, reproductive toxins, irritants, corrosives, sensitizers, liver toxins, kidney toxins, nerve toxins, agents that act on the hematopoietic (blood-forming) system, and agents that damage the lungs, skin, eyes, or mucus membranes. For the sake of consistency, "hazardous chemical" is defined the same way for both standards. Appendices A and B of the Hazard Communication Standard (included in this CHSP as Appendix 1) provide further guidance in defining the scope of health hazards and determining whether or not a chemical is to be considered hazardous. A list of definitions explaining basic hazardous chemical terms is provided in Appendix 3 of this CHSP.

Both the Laboratory Standard and the Hazard Communication Standard dictate that the physical hazards (e.g., flammability) presented by chemicals be addressed in the training programs. This CHSP addresses both the health and physical hazards attributed to certain chemical groups. However, more information on physical hazards is available in Pub-3000 [Chapter 7 (Cryogenic Safety), Chapter 12 (Fire Safety), Chapter 13 (Gases), and Chapter 20 (Seismic Safety)].

One class of hazardous chemicals is carcinogens. The term "select carcinogen" was introduced in the Laboratory Standard to refer to a subgroup of carcinogens for which special considerations are given in terms of controls, use, and pre-approval. Select carcinogens are discussed further in Section G.2.

\section{Hazard Assessment}

Hazard assessment is an evaluation made of chemicals present in the workplace to determine if they are hazardous. Note that the hazard determination must be made regardless of the potential for exposure. If there is potential for exposure, other than in minute, trace, or exempt cases (e.g., use of commercially prepared kits within which all the reagents are self-contained), then a hazard determination must be 
made. The hazard determination may declare that the potential for exposure is low and therefore the risk is low, and that additional control measures are not needed, but the exercise is still conducted and written down in the SSP.

Hazard assessment requires the evaluator to use the definitions provided in Appendix 3 of this CHSP, to refer to published lists of hazardous chemicals, and to utilize professional judgement in assessing the potential for workplace hazards.

Generally, any chemical known to be toxic, carcinogenic, or an irritant will be listed in 29 CFR 1910, Subpart Z (Federal OSHA Permissible Exposure Limits), or in California's list of OSHA-regulated chemicals and the American Conference of Governmental Industrial Hygienists' "Threshold Limit Values for Chemical Substances and Physical Agents in the Work Environment." Any remaining potential carcinogens are included in lists referenced by the Laboratory Standard identifying select carcinogens. Note that chemicals presenting only physical hazards (e.g., liquid ritrogen) often require greater scrutiny because they may not be included on any of the aforementioned lists.

Many products used in the work place are actually mixtures of different chemicals. Generally, a mixture is assumed to present the same health hazards as each of its components that comprise $1 \%$ or more of the mixture (in accordance with Hazard Communications Standard, as referenced in the Laboratory Standard). A mixture must be assumed to be carcinogenic if it contains a carcinogenic component in a concentration of $0.1 \%$ or more. The physical hazard potential of a chemical mixture must be evaluated on a case-by-case basis.

The Hazard Communication Standard does not require that a hazard determination be made for hazardous waste (e.g. soil and water samples), or for consumer products that contain hazardous substances if it can be demonstrated that the products are used in the same manner in the workplace as they are in normal consumer use (such that no greater exposure results). However, employees are reminded to use good judgement when using all chemicals, even common household bleach. 


\section{Operational Safety Procedures}

Certain laboratory or shop operations require the development of detailed Operational Safety Procedures (OSPs). An OSP is required for any operation in which a malfunction has the potential to cause serious injuries or fatalities, environmental impact, significant property damage, or major interruption of Laboratory operations. The key elements that trigger the requirement for an OSP (rather than a SSP) are the gravity of the potential damage and the short time order in which that damage can occur. Examples of operations (chemical and nonchemical) requiring OSPs include:

- Class 3 and Class 4 laser systems and other high hazard non-ionizing radiation equipment

- Systems using ionizing radiation equipment or substances (e.g., X-ray machines, irrradiators, accelerators, and experiments using kilocurie amounts of radioactive substances)

- Systems using high voltage equipment

- Systems using toxic and/or pyrophoric gases (See list in Section D.3 or G.4)

- Use of extremely reactive and/or explosive substances (e.g., perchloric acid); [Note: See Section D.3 and G.6 for listings of reactive chemicals, and consult the Industrial Hygiene Group for applicability.]

- Operations requiring the use of glove boxes for safety reasons

- Procedures recommended as Biosafety Level 3 and 4

The OSP must be reviewed by multiple parties, assigned by their areas of expertise and responsibility. Generally the Toxic Substances Safety Subcommittee, Industrial Hygiene, and other mechanical, electrical, and/or fire prevention experts are involved in the review process. For information on the requirements for the preparation of a OSP, see the LBL Health and Safety Manual (Pub-3000). 


\section{Specific Safety Procedures}

Most laboratory and shop operations that utilize hazardous materials do not require OSPs. However, pursuant to the requirements of the Laboratory Standard, written safety procedures must indicate specific measures that will ensure worker safety. The Principal Investigato: or Lab/Shop Supervisor has the responsibility of preparing Specific Safety Procedures (SSPs) that describe (1) the specific hazards associated with a procedure/operation, and (2) the methods (i.e., safety procedures) for controlling those hazards.

Information and guidelines for writing SSPs are included in Appendix 4. A simple, fill-in-the-blank, two-page form serves as the SSP template. Note that the process of writing SSPs need not be unduly burdensome. Many laboratory operations can be lumped together such that only a few SSPS are required. The Industrial Hygiene Group is available to assist supervisors in drafting their SSPs. Also, standardized SSPs for common laboratory operations/procedures may be shared among supervisors.

Examples of operations and procedures requiring SSPs include:

- Any hazardous chemical operation not requiring an OSP

- Processes that utilize hazardous chemicals at certain phases of the operation (e.g., some steps in tissue culturing and protein extractions, and the use of high pressure liquid chromatography)

- Use of Class 2 laser systems

- Use of sealed radioactive sources and radionuclides

Whereas an OSP must be submitted to, reviewed by, and approved by the applicable Safety Review Subcommittee and the Division Director an SSP is intended as an internal document. It is to be reviewed by the Principal Investigator/Supervisor at least annually and followed by all personnel performing the specific tasks/operations for which it was written. 


\section{Variances from Safety Guidelines}

A variance from LBL safety guidelines may be requested in writing from the EH\&S Division. The experimenter must first seek the concurrence of his/her division director. Variances from DOE-prescribed orders and standards require DOE approval, and must be requested through the Associate Laboratory Director for Operations.

Requests for variances must include the following elements:

- An enumeration of the specific criteria, rules, or procedures with respect to which the variance is requested;

- An explanation of the need to perform the experiment in the proposed manner;

- A description of the experimental apparatus;

- A description of the measures to be taken to ensure that the variance will not compromise safety; and

- A statement of the period during which the variance is to be in effect.

The EH\&S Division Director may ask that the request for a variance be studied by the Safety Review Committee and its appropriate subcommittees. Approval or denial of a variance is given in writing by the Division Director of EH\&S after a thorough evaluation of the issues. Denial of a variance may be appealed to the Associate Laboratory Director of Operations.

If a variance is granted, EH\&S shall closely monitor the experimental operation for the duration of the variance to ensure that the conditions of the variance are met and that safety is not compromised. 


\section{CHEMICAL PROCUREMENT}

\section{Overview}

Laboratory and shop personnel responsible for ordering chemicals must be cognizant of the following requirements:

- Ordering of chemicals, including blanket order transactions, must be approved by the Principal Investigator, Shop Manager, or designated alternaie using the University of California Purchase Requisition (Appendix 5). After a blanket order is established, purchase requisitions are still required for each release against that order (i.e., for each subsequent chemical purchase). The purchase requisition is marked "Blanket Order" and must be sent to the Industrial Hygiene Group (Mailstop 26/109) to keep them informed of the types and amounts of chemicals being ordered. It is not necessary to send a copy of the blanket order requisition to the Purchasing Department.

- The individual ordering chemicals is responsible for declaring the hazardous properties of those chemicals on the hazard review section of the Purchase Requisition. The Purchasing Department will provide EH\&S with copies of all orders of potentially hazardous materials.

- MSDSs must be acquired for the work area in which the chemical will be used unless the MSDSs are already on file.

- Containers must not be accepted without an adequate identifying label (e.g., chemical identity, hazard warnings, manufacturer's name and address).

- Chemical purchases must be kept to the minimum volume required to sustain laboratory/shop operations without incurring significant operational interruption so as to avoid high waste disposal costs at a later date.

- Certain chemicals are controlled at the purchasing phase of procurement and may be subject to special hazard review by EH\&S (see Section D.2). 


\section{Purchase-Controlled Substances}

Some substances at LBL are controlled at the purchasing phase of procurement. These substances are controlled either prior to (1) actual placument of the order (e.g., for drug precursors), or (2) delivery of the substance to the individual who ordered it (e.g., radioactive substances). Purchase-controlled substances include radioactive substances, toxic gases, reactive (unstable) chemicals, and drug precursors.

Various departments in the EH\&S Division are responsible for reviewing and/or pre-approving specific substances prior to their delivery to the user. The chemicals that the Industrial Hygiene Group is involved in reviewing are reactive chemicals, toxic gases, carcinogens, and any other extremely hazardous chemicals.

\section{Industrial Hygiene Pre-Approval/Review}

The Industrial Hygiene Group receives copies of all purchase requisitions and reviews the chemicals ordered to identify those parties who are ordering extremely hazardous materials. The Group periodically audits operations to ensure that provisions are in place to mitigate the hazards. In some cases an Operational Safety Procedure (OSP) is required (e.g., for toxic and pyrophoric gases); usually a Specific Safety Procedure (SSP) is sufficient (e.g., for carcinogens and reproductive toxins).

\section{Reactive Chemicals}

Examples of reactive chemicals of greatest concern to the Industrial Hygiene Group is given below:

$\begin{array}{ll}\text { benzoyl peroxide } & \text { phosphorus (white) } \\ \text { copper azide } & \text { picric acid } \\ \text { diethyl ether } & \text { potassium } \\ \text { dimethyl ether } & \text { sodium } \\ \text { isopropyl ether } & \text { sodium azide } \\ \text { lead azide } & \text { triethyl aluminum } \\ \text { lithium aluminum hydride } & \text { trimethyl aluminum } \\ \text { perchloric acid } & \end{array}$




\section{Toxic and Pyrophoric Gases}

The use of hazardous gases at LBL is strictly controlled. Hazardous gases include corrosive, flammable, pyrophoric, and/or toxic gases. The use of any hazardous gas must be strictly reviewed to ensure that appropriate and adequate safety systems are in place. It is the responsibility of Division Directors to ensure that this review has been carried out before any new equipment is used or new procedure is adopted.

Special additional policies are in force for hazardous gases designated as highly toxic or pyrophoric (spontaneously combustible) by the Uniform Fire Code. These gases are controlled items, and their purchase requires the signature of an authorized person (typically a Principal Investigator or senior Laboratory official) and the Industrial Hygiene Group.

An approved Operational Safety Procedure (OSP), which includes a hazard analysis, is required before any highly toxic or pyrophoric gas my be procured and delivered for use. (See the LBL Health and Safety Manual, Pub-3000, for instructions on the preparation of an OSP.) Under prescribed conditions, when very small amount of hazardous gases are used, the Industrial Hygiene Group may waive the requirement for an OSP.

Necessary safety equipment such as fume hoods or gas cabinets, safety showers, eyewash, and fire extinguishers must be present. In addition, the experimental apparatus must be properly installed and equipped with proper tubing, valves, ventilation for pumps, and other safety equipment as needed to handle the gas safely. Contact the Industrial Hygiene Group (Bldg. 26, Ext. 5829) for assistance.

Training in accordance with the OSP must be given to all persons operating the experiment apparatus. In general, it will be the responsibility of the Principal Investigator to provide this training. In addition, all persons subject to potential hazards from normal operation or perceivable accidents (e.g., fire fighters and other emergency crew personnel) m'ist likewise be appropriately trained. Personnel in adjacent work areas who do not cperate hazardous gas equipment must be trained in appropriate actions to take in the event of an alarm or emergency. 
Examples of highly toxic and/or pyrophoric gases.

\begin{tabular}{|c|c|c|}
\hline 1,3 butadiene & germane & phosgene \\
\hline arsenic pentafluoride & hydrogen cyanide & phosphine \\
\hline arsine & hydrogen fluoride & phosphorus pentafluoride \\
\hline boron trifluoride & hydrogen selenide & phosphorus trichloride \\
\hline bromine pentafluoride & hydrogen sulfide & phosphorus trifluoride \\
\hline bromine trifluoride & iodine pentafluoride & selenium hexafluoride \\
\hline carbonyl fluoride & methyl bromide & silane \\
\hline chlorine & methyl chloride & silicon tetrafluoride \\
\hline chlorine trifluoride & methyl silane & stibine \\
\hline cyanogen & nickel carbonyl & sulfur tetrafluoride \\
\hline cyanogen chloride & nitric oxide & sulfuryl fluoride \\
\hline diborane & nitrogen dioxide & tellurium hexafluoride \\
\hline dichlorosilane & nitrosyl chloride & tungsten hexafluoride \\
\hline fluorine & oxygen difluoride & vinyl chloride \\
\hline
\end{tabular}

Note: Because codes and regulations frequently change, this list in not intended to be complete. The Industrial Hygiene Group should be consulted if information is needed on the classification of a specific gas not listed above. 


\section{E. STANDARD HYGIENE AND SAFETY PRACTICES}

\section{General Principles}

The number of hazardous chemicals and the number of reactions among them is so large that previous knowledge of all potential hazards cannot be assumed. Therefore, when the chemical properties of a material are not fully known, it should be assumed hazardous and used in as small quantities as possible to minimize exposure and thus reduce the magnitude of unexpected events.

The following general safety principles should be observed when working with chemicals:

- Substitute less toxic materials whenever possible.

- Minimize all chemical exposures through the use of engineering (e.g., lab hoods), administrative (e.g., access controls) and personal protective controls (e.g., gloves).

- Obtain and read the Material Safety Data Sheet and other hazard information on all chemicals (i.e., solids, liquids, and gases) used to support the laboratory operations.

- Confine long hair and loose clothing when working in the laboratory/shop.

- Be knowledgeable in the use of emergency equipment such as eye washes, showers and fire extinguishers and know how to obtain additional help in an emergency.

- Carefully label or cross-reference every secondary container with the identity of its contents. Appropriate hazard warnings will be required if more than one person will be using the secondary container or if the container's contents are not fully used in the same workday.

- Utilize equipment only for its designed purpose. 
- Keep the work area clean and orderly.

- Observe the administrative controls set forth that restrict and control specific areas (e.g., radiation "control" areas, carcinogen "designated" areas, or "regulated" areas, etc).

- Determine the compatibility of chemicals and store incompatibles separately (refer to Section E.9).

- Provide a means of containing the material if equipment or containers should break or spill their contents (i.e., secondary containment).

- Limit the volume of volatile or flammable material to the minimum needed for short operation periods. Refer to Section G.7 for a specific discussion on flammable and combustible liquids.

- Position and clamp reaction apparatus in order to permit manipulation without the need to move the apparatus until the entire reaction is completed. Combine reagents in appropriate order, and avoid adding solids to hot liquids.

- Ensure that all chemical storage cabinets and racks, and all laboratory equipment using hazardous materials, are seismically braced in accordance with the best structural engineering practices.

- When mixing acid and water, ALWAYS ADD the ACID to the WATER so as to avoid reactions and splattering.

- Follow the requirements in the LBL Health and Safety Manual (Pub-3000), Chapter 20, Pressure Safety, if systems that can generate pressure or are operated under pressure are involved.

\section{Basic Hygiene}

- Use respiratory protective equipment in accordance with the established respiratory protection program. See LBL Health and Safety Manual (Pub-3000), 
Chapter 19. Note: Respiratory protection is not the first line of defense; the need to use such protection is reviewed by the Industrial Hygiene Group.

- NEVER use mouth suction to pipet chemicals or to start a siphon; use a pipette bulb or an aspirator to provide vacuum.

- Wash hands with soap before leaving the laboratory/shop area. Never wash with solvent materials.

- Store, handle, and consume food in areas free of hazardous substances and hazardous equipment to prevent contamination.

- Do not store food or drink in the same refrigerator, ice chest, cold room, etc., as used to store chemicals, reagents, biologics, or animal products. Dedicate separate equipment for food and drink storage and prominently label the equipment declaring the intended use.

- Do not smoke, chew gum, or apply cosmetics in areas having chemical or biological hazardous agents present.

- Do not use glassware or utensils that have been used for laboratory/shop chemical use to prepare or consume food or beverages.

- Keep laboratory bench tops clean, and clean up the area at the end of an analysis or procedure.

- Clean the surfaces of containers of drips and wipe bottle rings off the bench top.

- Remove jewelry from wrists and hands to prevent (1) chemicals from collecting on the jewelry, (2) contact with electrical sources, (3) catching on laboratory or shop equipment, and (4) damage to the jewelry itself. 


\section{Personal Protective Equipment}

The use of personal protective equipment is needed to complement the variety of engineering and administrative controls present in the laboratory and shop environment. See LBL Health and Safety Manual (Pub-3000), Chapter 19 for more information on personal protective equipment The following is a listing of minimum guidelines for laboratory and shop personnel:

\section{Body and Footware}

- Wear appropriate clothing, including a protective apron or laboratory coat to protect against chemical splashes or spills, and temperature extremes. Use protective apparel, including face shields, gloves, and other special clothing or footwear as needed.

- If apparel becomes contaminated, remove and place inside sealed bags. Do not launder at home if the coats/aprons become contaminated with hazardous chemicals, human blood products, or infectious agents.

- Confine loose apparel.

- Remove laboratory or shop coats/aprons prior to leaving the work area. Do not wear them into lounge areas, cafeteria, etc. The goal is to confine contaminants to the specific areas in which their presence is anticipated.

- Do not wear open-toed shoes or sandals in the laboratory/or shop. Note: Some shop areas require wearing steel-toed (safety) shoes.

\section{Gloves}

- Gloves should be worn whenever working with hazardous chemicals, rough or sharp-edged objects, or very hot or very cold materials.

- Select gloves based on the material(s) being handled, the particular hazard(s) involved, and their suitability for the procedures being conducted. In order to select the appropriate glove, refer to the glove selection charts in Appendix 6. 
- Inspect gloves prior to use and change them often based on the frequency of use and the permeability to the chemical(s) handled.

- All gloves are eventually permeated by chemicals. Inspect gloves before each use for discoloration, punctures, and tears.

\section{Eye Protection}

- Safety glasses are required in all areas where chemicals are in use. The safety glasses should be impact resistant eye glasses with side shields.

- Wear goggles and/or face shields when there is danger of splashed chemicals or flying particles. (e.g., when pouring or mixing chemicals).

- Contact lenses alone (i.e., without safety glasses) are prohibited when handling corrosive chemicals.

\section{Respirators}

Respirators should not be needed in a normal laboratory or shop setting. However, if engineering and administrative controls cannot assure that concentrations of airborne hazardous chemicals are maintained below Action Levels (ALs), Permissible Exposure Levels (PELs), and Threshold Limit Values (TLVs), or when atmospheric conditions are unknown, respiratory protection is required. The Industrial Hygiene Group will determine the need for respiratory protection.

The LBL Respiratory Protection Program is established to insure a healthful working environment for LBL employees through the proper medical screening, fit testing, and training in the use of respiratory protective equipment. All use of respirators at the LBL comes under the requirements of the LBL Respiratory Protection Program, including routine and non-routine operations, emergency responses, and work in confined spaces. Respirators must be approved and obtained through the Industrial Hygiene Group. 


\section{Unattended Operations and Working Alone}

In general, it is prudent to avoid working in hazardous operations alone. Arrangements should be made between individuals working in separate hazardous areas off-shift to cross-check each other's safety periodically. Experiments known to be hazardous shall not be undertaken by a worker who is alone in a laboratory. At least two persons must be present.

Precautions should be taken for laboratory or shop operations that are carried out continuously or overnight. Operations should be designed to be safe, and plans should be made to avoid hazards in case of failure. If possible, make arrangements for routine inspections of an on-going operation, leave the lights on, and leave an appropriate sign on the door.

Operations requiring cooling water shall employ monitoring devices that will shut the operation down in the event of water supply failure.

\section{Housekeeping}

There is a definite relationship between safety performance and orderliness in the laboratory or shop. Work areas should be kept clean and free from obstructions. Cleanup should follow the completion of any operation or at the end of each day.

Hazardous chemical wastes shall be appropriately disposed of according to established guidelines discussed in LBL publications ["Guidelines for Generators of Hazardous Chemical Waste at LBL and Guidelines for Generators of Radioactive and Mixed Waste at LBL" (Pub-3092) and "Medical and Biohazardous Waste Generator's Guide" (Pub-3095)]. Old containers and chemical wastes should be disposed of promptly and not be allowed to accumulate in the laboratory or shop.

Spilled chemicals shall be cleaned up immediately and disposed of properly. Spills of large quantities of chemicals where there is the potential for personal injury, environmental impact and property damage shall be reported to the Fire Department for response. Dial Ext. 7911 to report such incidents. 
In addition, adhere to the following general housekeeping rules:

- Do not use stairways and hallways as storage areas.

- Clean up nonhazardous spills immediately.

- Maintain access to exits, emergency equipment, and other control equipment.

- Store equipment and chemicals properly and avoid clutter.

\section{Use of Glassware}

- Use careful handling and storage procedures to avoid damaging glassware.

- Use adequate hand protection (e.g., leather gloves) when inserting glass tubing into rubber stoppers or corks or when placing rubber tubing on glass hose connections. Tubing should be fire polished or rounded and lubricated, and hands should be held close together to limit movement of glass should fracture occur. The use of plastic or metal connectors should be considered.

- Do not attempt glass-blowing operations unless proper annealing facilities are available.

- Handle vacuum-jacketed glass apparatus with extreme care to prevent implosions. Equipment such as dewar flasks should be taped or shielded. Only glassware designed for vacuum work should be used for that purpose.

- Use hand protection (i.e., tear-resistant gloves) when picking up broken glass.

- Provide a separate marked trash receptacle or sharps container for broken glass. Glass must not be deposited in containers of wastepaper, rags, and other discarded materials.

- Provide proper instruction on the use of glass equipment designed for specialized tasks that can represent unusual risks for an inexperienced user. 
(For example, separatory funnels containing volatile solvents can develop considerable pressure during use.)

\section{Transport of Hazardous Materials}

It is against federal regulations to transport hazardous material off-site (UC campus areas are considered off-site) without (1) an appropriate manifest of the contents of the container, and (2) proper packaging for the hazardous material as required by its nature.

Synthesized materials that need to be transported from LBL to an off-site location will require the same manifesting and packaging requirements as do stock chemicals. A summary of hazard information is required to accompany the material prior to packaging and transport. This information should include: the chemical and physical hazards, reactivity warnings, and spill response guidelines.

Transportation of hazardous materials by private vehicle, the LBL bus, or nondesignated LBL vehicles is not permitted because of the possibility of spillage or breakage of the container and resulting risk of injury to personnel and damage to property.

LBL personnel on campus should order stock hazardous materials from the LBL Building 7 storeroom for delivery on campus. Or, with proper authorization, they may order hazardous materials from the chemical storeroom in Latimer Hall at UC Berkeley.

\section{Labeling}

\section{Area Entrances}

Entrances to a laboratory or shop using hazardous materials or having hazardous operations must be labeled with the following information:

- "Caution"/"Danger"

- Key Hazard Pictograms

- Special Procedure Information 
- Emergency Contact Information

For information on available Pictogram Hazard Keys, Refer to Appendix 7. Additional signs may be required for certain operations such as lasers, microwave sources, radioactive materials, etc.

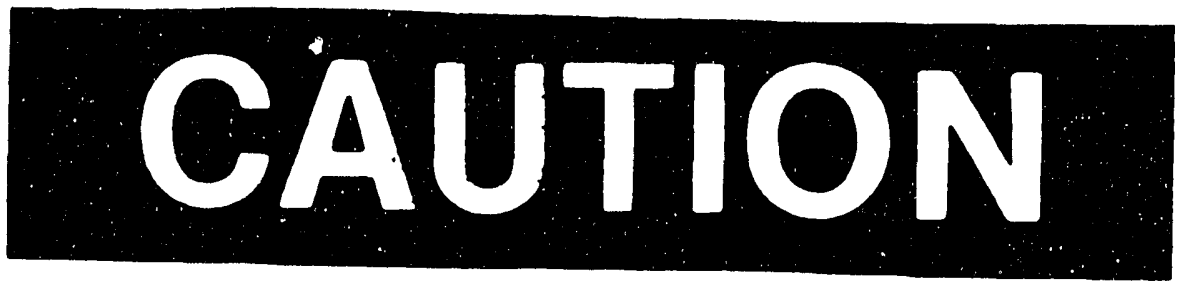

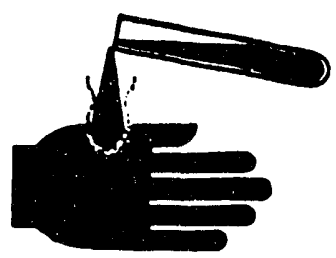

CORROSIVE
MATERIALS

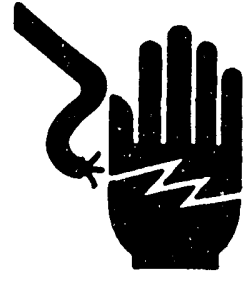

ELECTRICAL HAZARD

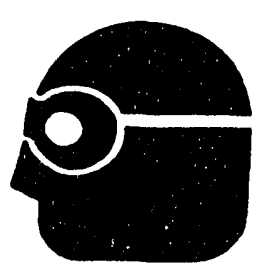

WEAR SAFETY GLASSES

ADMITTANCE TO AUTHORIZED PERSONNEL ONLY SPECIAL MHOCEDUAES ON MFONMATION

FOR EMERGENCY ASSISTANCE:

VISITORS ANO PERSONNEL NOT ASSIONFD TO THIS AREA

\begin{tabular}{|l|l|l|l|l|}
\hline CONTACT & NAWE & OFFICE & PHONE & HOME FHONE \\
\hline FOR EMTAT ON AOYICE & & & & \\
\hline IN EMERGENCY & & & & \\
\hline IN EMERGENCY & & & & \\
\hline
\end{tabular}




\section{Container Labeling}

As part of the Hazard Communication Standard, hazard warning labels or tags are required on the original shipping containers and any container subsequently used for storage. The label must:

- show the name of the material;

- provide hazard warning information appropriate for employee protection; and

- be legible and prominently displayed.

Labels on shipping containers must also include the name and address of the chemical manufacturer, importer, or other responsible party. Labels on shipping containers provided by the manufacturer are required to provide all the appropriate information and must not be removed.

All containers (including lab glassware, safety cans, plastic squeeze bottles) must have labels that identify their chemical contents. Labels must also contain information on the hazards associated with the use of the chemical. Exceptions to this requirement are secondary containers used by one person within his/her workshift. Labels on containers that an employee fills for his/her personal use during a single workday are not required to show hazard warnings, but the identity of the material must be shown.

Precautionary labels are available from LBL stock (LBL Stock Catalog Section 4280) for many of the common chemicals. Refer to Appendix 7.

\section{Storage of Chemicals}

The separation of chernicals (both solids and liquids) during storage is necessary to reduce the potential for unwanted reactions by accidental mixing. Additional specific information on chemical storage is provided in Appendix 8. Use either distance or barriers (e.g., trays) to isolate chemicals into the following minimum classifications: 
- Solvents, which include flammable liquids and halogenated hydrocarbons (e.g., acetone, benzene, ethers, alcohols)

- Inorganic acids (e.g., nitric, sulfuric, hydrochloric, and perchloric). Note: Treat acetic acid as a flammable liquid

- Bases (e.g., sodium hydroxide, ammonium hydroxide)

- Oxidizers

- Poisons

- Explosives or unstable reactives, such as picric acid. Store separately outdoors in flammable storage cabinets

The following storage rules apply to all LBL facilities:

- Store all hazardous liquid chemicals in secondary containers that are chemically resistant and unbreakable.

- Examine stored chemicals that form peroxides every few months for deterioration, integrity and expiration date (see Appendix 8).

- Limit the amount of chemicals permitted for storage to amounts that are as small as practical.

- Avoid exposure of chemicals to heat or direct sunlight.

- Do not use fume hoods as storage areas for chemicals. Chemicals temporarily housed in fume hoods should be kept to a minimum and should not block vents or alter airflow.

- Install lips, strips, or bars across the width of reagent shelves to restrain the chemicals in case of earthquake. 
- Do not store chemicals in a refrigerator used for food storage. Refrigerators used for storing chemicals must be appropriately identified by placing the following label on the door (labels may be obtained from EH\&S or from the Building? Storeroom).

\begin{tabular}{|c|}
\hline CAUTION \\
FOOD SHALL \\
NOT BE \\
STORED \\
IN THIS UNIT \\
\hline
\end{tabular}

\section{Spill Response}

The following actions should be taken when responding to chemical spills that have the potential of personal injury, environmental impact, and property damage:

- Evacuate people from the area.

- Isolate the area.

- If the material is flammable, turn off ignition and heat sources.

- Call the Fire Department, Ext. 7911, for assistance and notify the area supervisor.

- Wear appropriate personal protective equipment during cleanup.

- Pour Sorb-all or an appropriate neutralizing agent on the spill.

- Clean up and place waste in plastic bags for disposal.

- Decontaminate the area after gross spill cleanup if required.

- Follow waste labeling requirements outlined in in Pub-3092, Pub-3093, and Pub-3095 (Refer to "References," Section L). 
The following tables provide phone numbers for emergencies (including spills) and spill clean-up materials that are available from Stores (Building 7).

\section{Emergency Numbers}

All

Location

Emergencies

Radioactive or Other

Hazardous Spills or Leaks

LBL On-Site Bldgs.

incl. Campus Bldgs.

7911

7911

Donner \& Calvin Labs

All other LBL Off-Site

Bldgs.

7911

(Bldgs. 901, 934, 935, \&

936)

All non-LBL Campus

9-911

9-911

Bldgs.

Chemical Spill Clean-up Materials From Stores

\begin{tabular}{lc}
\hline \multicolumn{1}{c}{ Description } & Catalog No. \\
\hline Flammable Solvent Spill Kit & $4240-67879$ \\
\hline Flammable Solvent Absorbant & $4240-67880$ \\
\hline $\begin{array}{l}\text { Acid Spill Kit } \\
\text { Acid spill absorbant }\end{array}$ & $4240-67881$ \\
\hline Caustic (base) Absorbant & $4240-67882$ \\
\hline $\begin{array}{l}\text { Safety Equipment Kit (contains scoops, sponge, } \\
\text { safety glasses, disposal bags, etc.) }\end{array}$ & $4240-67884$ \\
\hline \begin{tabular}{l} 
Cabinet spill kits \\
\hline
\end{tabular} & $4240-67885$ \\
\hline
\end{tabular}




\section{Disposal of Chemicals}

All LBL employees, participating guests, and contractors using hazardous chemicals are responsible for disposing of these chemicals safely. Federal and state regulations mandate strict disposal procedures for chemicals. In order to comply with these regulations all persons using LBL facilities must observe these procedures. And, all persons directly involved in the disposal of hazardous waste must be trained in LBL's procedures (EH\&S Course \#343).

\section{Routine Disposal of Chemicals}

Disposal of toxic and/or hazardous chemicals down sinks or sanitary-sewer drains is prohibited. However, in some buildings disposal of certain acids and bases is allowable because the building is equipped with a pH adjustment tank. Disposal of any other chemicals down sinks is done only under carefully controlled conditions after consulting the Environmental Protection Group.

Wastes shall not be accumulated for more than 90 days at "satellite accumulation areas," 60 days at "waste accumulation areas," or 7 days if it is medical/biohazardous waste or full sharps containers (30 days if the medical/biohazardous waste is frozen). See Section L for LBL publications that provide specifics on waste handling (Pub3092, Pub-3093, and Pub-3095) and/or contact the Environmental Protection Group (Ext. 5251) for advice and guidance.

In using chemical waste storage containers, certain procedures must be observed, "as conditions for waste pickup and disposal." The procedures and/or policies are listed below:

- Establish prior to disposal what type of waste it is: hazardous, radioactive, "mixed," medical/biohazardous, or merely "trash."

- Incompatible chemicals must not be mixed in the same container (i.e., acids should not be mixed with bases, organic liquids should not be mixed with strong oxidizing agents, and flammable liquid wastes must be separated from chlorinated wastes). This is referred to as "segregation." 
- Chemical containers must be "separated" in compatible groups. Containers of flammable liquids, chlorinated hydrocarbons, acids, bases, oxidizing agents, other liquids, solids, and explosive materials must be separated such that containers of incompatables are not stored together.

- Waste oils must be collected in drums. Disposal will be arranged by the Transportation of Materials Group. Ext. 5404.

- Flammable liquid wastes should be stored in approved 5-gallon containers (LBL catalog No. 7960303444). All other liquid wastes should be stored in any appropriate, properly labeled, leakproof containers.

- Leaking containers of any sort will not be accepted.

- Dry materials (gloves, wipes, pipettes, etc.,) must be securely contained in plastic bags and overpacked in a cardboard box. Packages that are wet or have sharp protruding objects will not be accepted for pick up.

- Unknown chemicals require special handling. The responsible department must make every effort to identify the material that is to be disposed of.

- Each breakable container must be properly boxed. Plastic bag all bottles, then place in a sturdy container and use an absorbent cushioning material that is compatible with the chemicals.

- Each primary container must be labeled with contents, amount, physical state, and the percentage breakdown when dealing with a mixture.

- Each box must have a complete list of contents or a description written on an official EH\&S hazardous materials packing list. Blank packing lists are available from the EH\&S office. Call Ext. 5251 to place an order.

- For safety purposes, boxes must be of a reasonable size and weight so the one person is able to handle them. Boxes that exceed 45 pounds or 18 inches on a side cannot be safely handled by one person and will not be acceptable for pick up. 
- Check with EH\&S on feasible and allowable methods to reduce or alter the hazardous properties of anticipated wastes. For example, acids and bases may be neutralized and certain toxins can be oxidized or adsorbed.

- Place contaminated sharps (e.g., blades, Pasteur pipettes, syringes) into "sharps containers."

\section{General Housekeeping Rules}

- Ensure that storage areas, or equipment containing large quantities of chemicals, are secure from accidental spills.

- Recycle unused laboratory chemicals as much as possible.

- Do not place hazardous chemicals or contaminated labware (e.g., glassware, gloves, etc.) in salvage or garbage receptacles.

- Do not pour chemicals onto the ground.

- Do not dispose of chemicals through the storm-drain system or sewer drain. 


\section{F. HAZARD CONTROL MEASURES}

This section describes how to determine when controls are needed, the general types of controls that can be used to reduce employee exposures to hazardous chemicals, back-up controls such as safety and emergency equipment, and equipment maintenance protocols.

Employee exposures to potential hazards can be controlled through the following basic approaches:

- Engineering controls

- Administrative controls

- Personal protective equipment

All three of these approaches may be used for a single procedure depending upon the circumstances.

\section{Criteria for Control Measures}

Exposures by inhalation of airborne contaminants (gases, vapors, fumes, dusts, and mists) must not exceed (1) the levels listed in the latest edition of Threshold Limit Values (TLVs) of Airborne Contaminants published by the American Conference of Governmental Industrial Hygienists, (2) OSHA Permissible Exposure Limits (PELs), which are comparable to the TLVs, and (3) the ACGIH and/or OSHA Short Term Exposure Limits (STELs). These occupational exposure limits are normally published on the manufacturer's Material Safety Data Sheets or available through the Industrial Hygiene Group.

The TLV and PEL levels refer to airborne 8-hour, average concentrations of substances and represent conditions under which it is believed that workers may be repeatedly exposed without adverse effect. The STELs represent 15-minute average concentrations that represent the maximum peak concentrations to which workers can be exposed. Not all chemicals have PELs or TLVs; and, not all chemicals that have 8-hour TLVs and PELs, also have STELS. STELs are particularly important in protecting workers from toxins that can quickly cause damage, i.e., acute toxins. The Industrial Hygiene Group conducts exposure 
monitoring and evaluates exposures based the occupational exposure limits (See Section I).

In all cases of potentially harmful exposure, feasible engineering or administrative controls must first be established. In cases where respiratory protective equipment, alone or with other control measures, is required to protect the employee, the protective equipment must be approved by the Industrial Hygiene Group for each specific use.

The need for controls to reduce employee exposures during a specific operation is usually based upon a determination of the potential for exposure during the operation. This determination may be made by several different parties: The Industrial Hygiene Group and/or another health and safety professional specifically assigned to make this determination, the operator or scientist, or supervisor. In general, this determination involves evaluating the operation in terms of the following criteria to determine how serious the potential for exposure is and whether controls are needed. The criteria considered include:

- Length of employee exposures (full shift vs. short-term)

- Types of substances being handled or used including the hazardous properties and physical and chemical properties

- The nature of the operation or procedure (i.e., how easily are aerosols or vapors generated).

- Presence of existing controls to reduce exposures

- Number of employees involved

- Quantities of chemical being handled

- Routes of exposure (i.e., how the chemical enters the body)

- Occupational exposure limits 


\section{"Rules of Thumb"}

The following rules of thumb are helpful. GENERALLY,

- When diluting concentrated acids and bases, use a hood.

- When using volatile toxic substances use a hood.

- When using select carcinogens, reproductive toxins, or highly toxic compounds, use a hood.

-When conducting procedures that generate particulates (e.g., dust) or liquid aerosols (e.g., when vortexing) of even moderately toxic chemicals, use a hood.

- When synthesizing or reacting chemicals, use a hood.

- When using odiferous compounds (even if they are relatively safe), use a hood.

- When using both infectious agents and hazardous chemicals together, use a properly equipped biological safety cabinet.

Situations with the potential for significant exposures should be redesigned or controlled by installing lab hoods, other exhaust ventilation, or other controls. This approach is intended to maintain exposures at low levels once the operation has been installed in the laboratory. If exposures are still significant after the operation has been implemented in the laboratory, changes in the work practices or personal protective equipment are used to provide additional protection for employees.

In general, five criteria determine if additional controls are needed:

- Employees have concerns and/or symptoms

- Changes in operation or the facility alter the needs 
- Employee exposure monitoring results indirate potential or actual exposure problems

- Problem areas prone to spills and/or accicients are identified

- An industrial hygienist determines it is prudent and justifiable based on the hazard potential and/or applicable codes/standards

See also Sections $G$ and I for more information on assessing hazards to determine which controls are best.

\section{Engineering Controls}

The use of engineering controls (e.g., lab hoods) often involves permanent changes to the facility that reduce or remove the hazard from the area, such that the hazardous substances cannot diffuse into the surrounding areas.

No laboratory or shop area can rely on one particular type of control technology to ensure that exposures to hazardous chemical agents are as low as reasonably achievable. However, the primary and most effective approach is through the use of engineering controls. Complementing the engineering controls are the correct combination of administrative procedures and use of personal protective equipment.

\section{General Ventilation}

The general ventilation system, referred to as the heating, ventilation and air conditioning (HVAC) system, should not be used alone to control employee exposures. The HVAC system is not a type of local exhaust ventilation. However, certain allowances for how the HVAC system operates are critical to worker safety.

Recirculation of contaminated exhaust air in laboratories or shop areas using toxic, corrosive, flammable or other hazardous agents is prohibited.

Laboratories are to be designed such that air passes through once only.

Occasionally it may be necessary to provide additional temperature control for a 
single laboratory room by recirculation of the air in the room. This is permissible as long as the air does not recirculate to other rooms.

Laboratories facilities using highly volatile carcinogens and acute toxins must be designed so that a negative pressure differential exists between the laboratory and the exit corridor(s) servicing the laboratory, thus containing accidental releases to the room in which they were generated.

\section{Local Exhaust Ventilation (e.g., hoods)}

Lab hoods are the most common type of local exhaust ventilation. Generally, hoods should be considered as backup devices that can contain and exhaust toxic, offensive, or flammable materials when the design of an experiment fails and vapors or dust escape from the apparatus being used.

Self-contained (ductless) re-circulation hoods are not allowed at LBL if their use is intended to control hazardous chemicals; they may be permitted for non-toxic, odiferous work such as for some clinical laboratory tests. Consult the the Industrial Hygiene Group for more information.

Hoods are not regarded as a means for disposing of chemicals, and they are not intended for storage of chemicals. Materials used in hoods should be kept to a minimum and should not block vents or alter airflow patterns.

Hoods should be evaluated by operators prior to and during each use by means of simple visual indicators (such as mylar strips or flow monitors) for adequate air flow. User-controlled performance of a hood depends upon such factors as the placement of equipment in the hood, room drafts from open doors or windows, turbulence caused by persons walking by, and the presence of the user in front of the hood. Keep apparatus back from the front edge of the hood to reduce the potential for contaminant release.

During operations, minimize the face opening of the hood to improve the performance of the hood. Reducing the opening in the laboratory hood may also provide some protection from explosives due to chemical reactions, overpressurization, etc. Except when adjustments of apparatus within the hood are 
being made, the hood sash should be kept at or below the marked "safe sash height. "If the hood does not have a bypass grill then the sash should be left open at least six inches to avoid the flow from being choked off. Sliding sashes should not be removed from horizontal sliding-sash hoods.

Mechanical ventilation must remain in ope ttion at all times when hoods are in use and for a sufficient time thereafter to clear hoods of airborne hazardous substances. When mechanical ventilation is not in operation, hazardous substances in the hood must be covered or capped off. Laboratory workers should be prepared for the event of ventilation failure or other unexpected occurrence such as fire or explosion in the hood.

Hoods must be inspected frequently and cleaned as necessary to ensure adequate air flow and the prevention of residue buildup. The Industrial Hygiene Group conducts an annual ventilation survey and posts flow rates and the date of test.

The end user is not solely responsible for the correct design and performance of laboratory hoods. Usually, Plant Engineering plays a role in the design of hoods, and Construction \& Maintenance installs the hoods. The Industrial Hygiene Group checks the performance of the hoods at least annually and whenever asked to check for possible hood performance problems. Nonetheless, all parties should be aware of the following rules and specifications that apply to lab hoods:

- Provisions must be made for adequate make up air for all hoods that are used in a laboratory.

- General room air flow near the hood should not be turbulent and should be relatively uniform throughout the laboratory/shop.

- Laboratory-type hood face velocities must be sufficient to maintain an inward flow of air at all openings into the hood under operating conditions. Air flow into hoods depends upon configuration but must be at a minimum average of at least 100 linear feet per minute (lfpm) face velocity with a minimum of $70 \mathrm{lfpm}$ at any point, except where more stringent special requirements are identified. 
- Generally, the face velocity must be obtainable with the movable sashes opened at least 24 inches. Where the required velocity can be obtained by partly closing the sash, the sash and/or jamb must be marked to show the maximum opening at which the hood face velocity meets the requirements. Any hood failing to meet the requirements must be considered deficient in airflow and must be posted with placards, plainly visible, which prohibit use of hazardous substances within the hood.

- When sufficient quantities of flammable gases or liquids are used, or when combustible liquids are heated above their flash points, hoods that are not bypassed must have permanent stops installed that restrict closure of the sash so that sufficient airflow is maintained to prevent explosions. Concentrations in the duct must not exceed $20 \%$ of the lower explosive/flammable limit. (The Uniform Fire Code, Part VII sets forth requirements for the use of cryogenic fluids, and flammable and combustible liquids.)

- For new laboratory hood installations, a fire sprinkler head must be mounted on the inside of the hood. Newly purchased laboratory hoods and installed exhaust ducting for solvent operations shall be constructed of noncombustible materials to reduce the potential of damage should a fire occur within the workstation. Similarly, newly purchased laboratory hoods and exhaust ducting for corrosive applications shall be constructed from or coated with materials that are resistant to corrosive compounds.

- A minimum of 2.5 linear feet of hood space per person working at the hood is recommended.

- Laboratory hoods must be seismically braced to prevent toppling or sliding during an earthquake.

- Perchloric acid must be used in a closed system or within a specially designated acid fume hood with wash down systems to prevent the accumulation of explosive perchlorates in the fume hood. (Consult the Industrial Hygiene Group for perchloric acid hood specifications.) 
- Local exhaust systems that are used in the laboratory or shop environment must be designed by Plant Engineering in accordance with ACGIH, ASHRAE, NFPA, and other nationally recognized standards.

Two important specifications:

(1) Exhaust fan systems must be non-sparking if exhausting sufficient quantities of flammable vapors and corrosion resistant if handling corrosive vapors, and

(2) Exhaust stacks must be located in such a manner with respect to air intakes as to preclude the recirculation of laboratory or shop hood emissions within a building.

- Do not attach other local exhaust systems to existing fume hood exhaust ducts without consulting Plant Engineering.

- Both contaminant fume hoods and glove boxes may require an in-line high efficiency particulate air (HEPA) filter or scrubber to remove particularly hazardous substances from the air stream before being exhausted from the building

- Glove boxes generally operate under negative pressure (e.g., Class III biosafety cabinets), though some operate under positive pressure. In the case of positive pressure boxes, leaks can cause problems. Glove boxes should be thoroughly tested before each use and there should be a method of mcnitoring the integrity of the system (such as a shutoff valve or a pressure gauge designed into it).

\section{Biosafety Cabinets ("laminar flow hoods")}

Biosafety cabinets protect a laboratory from biologically hazardous agents that could cause laboratory-acquired infections. They are designed to provide for the control of airborne particulates and aerosols, within a combined space, through the use of directional airflow and high efficiency particulate air (HEPA) filters. 
Biosafety cabinets are divided into three classes, based upon their design and intended purpose:

Class I Cabinets: Designed to protect the operator and the environment through the use of an inward flow of unrecirculated air. They are sometime fitted with glove panels, gloves and air intake filters to increase the protection afforded the operator. This class of cabinetry is not intended to protect against contamination of materials being handled within the cabinet.

Class II Cabinets: Designed to protect the operator, environment and experiment from particulate contamination, including microorganisms, using an inward flow of room air through a front work opening, directional flow of HEPA-filtered air through the work area, and HEPA filtration of exhaust air. They should be used for work with infectious agents and some recombinant DNA work. Most laboratory personnel doing tissue culture work use Class II hoods.

Class III Cabinets: Totally enclosed ventilated cabinets of gas-tight construction. Work is done through attached rubber gloves. Class III cabinets are always operated under negative pressure, so that any leak, as in the case of a punctured glove, will be into the cabinet. They are designed for work with highly pathogenic organisms, but are sometimes used for work with carcinogenic chemicals. A minimum of 2 volume changes per hour and a negative pressure of 0.5 inches of water must be ensured for glove boxes.

\section{Additional Ventilation Considerations}

The following specific uses of laboratory/shop space or operations bear consideration for ventilation safety.

- Gas safety cabinets may be required for the use and storage of toxic and pyrophoric gases.

- Environmental rooms, whether refrigeration cold rooms or warm rooms for growth of organisms and cells, are usually closed air-circulation systems; therefore, evaluate the potential consequences of release of hazardous chemicals. 
- Magnetic latches or breakaway handles on doors of environmental rooms should be installed to allow a trapped person to dismantle the door.

- Environmental rooms shall be provided with emergency lighting (battery- or generator-powered) so that persons are never left in these areas without light.

- Volatile flammable solvents shall not be used in cold rooms due to fire hazards from ignition sources.

- The use of volatile acids should be avoided in cold rooms because such acids can corrode the cooling coils in the refrigeration system, which can lead to the development of leaks of hazardous refrigerants.

- Laboratory apparatus that may discharge hazardous vapors (e.g., vacuum pumps and distillation columns) must be vented to a local exhaust system.

\section{Administrative Controls}

\section{Correct Work Practices}

Changes in employee work practices for a specific operation can be an effective means of controlling exposures, especially for those operations in which individual variations can result in large differences in exposure levels. The purpose of using correct work practices is to design and implement the procedures and techniques that result in the least amount of exposures. The Industrial Hygiene Group will audit operations, evaluate work practices, and determine the need for changes that can reduce or better control exposures. This approach requires training to inform the employees of the correct procedures to ensure that all employees comply. Examples of this approach include the following:

- Allowing an experiment to dissipate any residual vapors before opening it to the lab environment. 
- Using good techniques to maximize the ability of a lab hood to capture emissions.

- Using tie correct tools and equipment to minimize the potential for spills and releases.

- Developing procedures for the correct sequence in mixing chemicals to minimize releases of vapors, e.g., whenever possible add powders to water rather that water to powers.

- Minimizing the quantities of volatile chemicals used.

- Keeping test chambers covered or enclosed during test reactions and other uses.

- Substituting a less toxic substance for a more toxic substance (e.g., using less concentrated acid or aqueous soap instead of organic solvent).

\section{Use of Restricted Areas}

Many of the substance-specific OSHA Standards for carcinogens describe the use of "regulated areas" as a means of hazard control. The regulated area is marked off, signs are placed, and access is controlled.

In similar fashion, the Laboratory Standard refers to "designated areas," to control the hazards associated with working with certain chemicals. A "designated area" means an area that may be used to work with "select carcinogens," reproductive toxins, or substances that have a high degree of toxicity. Basically, an area is "designated" to be the location in which such work may occur. A designated area may be the entire laboratory, an area of the laboratory, or a device such as a lab hood. The management must post a notification in the "designated area" to ensure that all employees in the area are informed of the hazardous substance(s) used in the area.

The purpose of the designated area is to heighten the awareness of working with specific substances and to restrict all activities involving these substances to the 
selected area. All handling and use of these substances should occur only in the designated area. The designated area may include the following controls:

- Well identified boundaries (usually a room or laboratory section).

- Signs indicating the designated area and the types of substances being handled.

- Entry restricted to authorized personnel.

- Special controls for minimizing exposures (e.g., lab hoods, enclosed systems).

- A special storage area for those substances separate from other materials being stored in the laboratory.

- The use of wet housekeeping techniques.

- Special training requirements regarding the hazards and controls.

- Special hygiene facilities (e.g., clean room/dirty room) with a walk-through shower for entering and exiting.

- Special procedures for entering and exiting.

See Section $G$ for more discussion of the use of designated areas.

\section{Personal Protective Equipment}

Personal protective equipment should be considered ancillary to other control measures, and users should understand which iy pe of equipment is best for their purposes. This type of hazards control requires that employees cooperate in using the equipment correctly whenever it is needed, and that they understand the limitations of the equipment. Protection such as goggles, face shields, gloves, aprons, and lab coats should be used, when needed, in addition to other controls such as those previously described. Respiratory protection should only be used if 
other controls are not feasible or adequate to achieve acceptable exposure levels. Proper selection of the correct personal protective equipment for a particular operation is very important. See Section E.3 for more information on personal protective equipment.

\section{Safety and Emergency Equipment}

\section{Equipment Guarding}

All mechanical equipment shall be adequately furnished with guards that prevent access to electrical connections, pinch points or moving parts, and all guards should be inspected before using equipment.

Employees are not to turn on, use, repair, or operate any hazardous laboratory or shop equipment unless trained and authorized to do so by the responsible supervisor.

\section{Shields}

Safety shields must be used for protection against possible explosions or uncontrolled reactions. Laboratory equipment must be shielded on all sides so that there is no line-of sight exposure of personnel. The sash on a chemical fume hood is a readily available partial shield. However, a portable shield must also be used, particularly with hoods that have vertical-rising sashes rather than horizontal-sliding sashes for operations having the potential for explosion such as:

- Whenever a reaction is attempted for the first time (small quantities of reactants should be used to minimize hazards).

- Whenever a familiar reaction is carried out on a larger than usual scale.

\section{Pressure}

Guidelines for the use of pneumatic and high pressure hydraulic equipment are given in the LBL Health and Safety Manual (Pub-3000), Chapter 20; however, the following are additional requirements for laboratory operations: 
- Reactions should never be carried out in, nor heat applied to, an apparatus that is a closed system unless it is designed and tested to withstand pressure.

- Pressurized apparatus shall have an appropriate relief device.

- If the reaction cannot be opened directly to the air, an inert gas purge and bubbler system should be used to avoid pressure buildup.

- All pressurized gas cylinders must follow procedures for safe equipment usage, handling, and storage discussed in Pub. 300, Chapter 13.

\section{Eyewash and Showers}

Eyewash fountains are required if a substance in use or stored presents an eye hazard (e.g., any corrosive and many irritants). The eyewash fountain must provide a soft stream or spray of aerated water.

Fully-plumbed safety showers must be provided when a rapid fire hazard exists, and in areas where one liter or more of any of the following are present: corrosives, eye irritants, and chemicals that are highly toxic via skin and/or eye contact. The shower must be capable of drenching the victim immediately in the event of an emergency. Hand-held wash units (at a sink or elsewhere) are allowed for the above-mentioned substances if the quantities present comprise less than one liter. Eye wash squeeze bottles are not acceptable, anytime.

Eyewash fountains and safety showers should be located close to each other so that, if necessary, the eyes can be washed while the body is showered. Access to these facilities must remain open at all times and reachable within 10 seconds or 100 linear feet, whichever is less. Showers shall not be located nearer than 25 inches from any wall and shall not be next to unprotected electrical panels or electrical equipment.

In case of accident, flush the affected part for at least 15 minutes. Report the accident to Health Services and the Laboratory/Shop Supervisor immediately. 
Eyewash and showers should be flushed at least weekly for three minutes to ensure that they are operating properly and that microbial contamination does not occur. At a minimum, eyewashes and showers shall be flushed monthly. Inspection tags should be filled out to document the testing. Note: Construction and Maintenance personnel check and flush the eyewashes and showers every three months; but, more periodic flushing is required by the users.

\section{Fire Extinguishers}

Laboratories and shops using hazardous chemicals must have an ABC-rated, dry chemical, fire extinguisher within $50 \mathrm{ft}$. of any exit for use on ordinary combustibles, flammable liquids, and electrical fires. If additional extinguishers are needed for an area, contact the Fire Department for information concerning recommendations and requirements.

\section{Flammable Liquid Storage Cabinets}

Not more than 60 gallons of Class I flammable liquids or Class II combustible liquids, nor more than 120 gallons of Class III combustible liquids may be stored in a storage cabinet. Storage of flammable liquids in excess of ten gallons must be in an UL listed, Factory Mutual (FM) approved, flammable liquid storage cabinet.

Flammable liquid storage cabinets should be used for the storage of flammable and combustible liquids only. Do not store corrosives, oxidizers, or reactive chemicals with flammable or combustible liquids. Cabinets must be labeled "Flammable Keep Fire Away."

(Note: A storage cabinet for flammable liquids is not fireproof, but only protects the contents from extreme temperatures for a limited time to allow evacuation of personnel and prompt entry of fire fighters.)

Refer to Section G.7 for more information on flammable/combustible liquids) 


\section{Preventive Maintenance}

Because of the wide variety of laboratory hoods (chemical fume hoods, biological safety cabinets, vented enclosures) and other local exhaust ventilation (vented waste containers, refrigerators, analytical instruments), the monitoring program for each piece of equipment should reflect the specific design and recommended operating practices. For laboratory operations involving hazardous substances, where exhaust ventilation is used for primary control of personal exposures, $a$ regularly scheduled ventilation system monitoring and maintenance program should be implemented. At LBL, the Industrial Hygiene Group checks every hood once per year.

Each laboratory facility should develop facility-specific safety procedures (SSPs) for the maintenance of engineering controls in their laboratories. Such procedures should include periodic inspections by EH\&S, with correction and follow-up as necessary. For example, it might be written that when a worker observes reduced hood velocity readings below 100 feet per minute, that they report the finding and call the Industrial Hygiene Group.

\section{Equipment Maintenance}

Proper equipment maintenance is important for safe, efficient operation. Equipment should be inspected and maintained on a regular basis.

\section{Hood Performance Evaluations}

- All hoods are checked by the EH\&S when they are first installed and annually thereafter, for adequate ventilation performance.

- Performance of a ventilation system must be checked whenever there has been a change in a system or location.

- Laboratory and shop ventilation equipment scheduled for maintenance or repair work must be cleaned and/or decontaminated. Maintenance workers have the right to refuse to do work if the area or equipment is not clear of hazards. 
- All ventilation systems need routine maintenance to prevent blocked or plugged air intakes and exhaust, loose belts, bearings in need of lubrication, motors in need of attention, corroded duct work, and component failure.

- Filters should be replaced periodically in certain types of ventilation systems, such as electrostatic precipitators and cyclones for dust collection.

- Monitoring devices such as a magnehelic or flow meters should be installed in all new ventilation systems and in systems that control certain highly toxic operations such as toxic gases or potent carcinogens.

- Class II biological safety cabinets must be "certified" by an outside contractor proficient in the process. The cabinets should be tested for filter integrity, air velocity, and air flow patterns (1) upon installation, and (2) at least annually thereafter, (3) when relocated, and (4) when HEPA filters are changed.

Note: A Class II biological safety cabinet does not require annual certification if it is not being utilized as a Class II cabinet, i.e., it is not being used to the level for which it was designed (when it is being used to protect the work product solely and not the worker). BUT the cabinet must be posted to alert workers. Place a caution sign on the hood with the following message:

\section{CAUTION}

This hood is not certified to protect workers from hazardous chemicals, biological agents, or radioactive substances.

DO NOT STORE or USE hazardous agents in this hood.

\section{Calibration of Corrosive, Pyrophoric and Toxic Gas Detection Systems}

Fixed gas detection systems may be required for operations using toxic gases that demonstrate poor human physiological warning properties. The specifications of 
the calibration technique for each system and the frequency of calibration must be documented. As a minimum, the specifications must be outlined in procedures associated with the operation of the apparatus (e.g., the OSP) with which the gasdetecting system is associated. It is recommended that calibrations of these systems be performed by an approved contractor or by other trained personnel. The Construction and Maintenance Department has resources available to assist in the formulation of maintenance contract.

It is the responsibility of every scientific program operating gas systems to pay the full costs for all calibration of gas-detection equipment associated with experimental apparatus or required by a particular scientific program. Costs for the calibration of equipment required to monitor building-air quality and ventilation systems or to control fire alarm transmission facilities are borne by the Construction and Maintenance Department.

\section{Equipment Decommissioning}

\section{Cleanup of Vacated Space}

Principal Investigators and Lab/Shop Supervisors are responsible for assuring that all laboratory and shop space occupied by their programs and/or activities is maintained free from undue hazards. This responsibility extends throughout the period of occupancy and when vacating laboratory and shop space. When vacating an area, all hazardous materials must be attended to such that all chemicals, radioactive materials and hazardous wastes are removed, transferred to new ownership, and/or properly disposed. Surface decontamination of floors, walls, ducts, etc. may also be necessary. The Environment, Health and Safety Division (Ext. 5251) can provide assistance in making radiological surveys and removing chemicals and waste.

If laboratory or shop premises are left in an environmentally unacceptable state, it may be necessary to obtain the services of outside contractors to identify and dispose of unidentified chemicals and waste. Should this be necessary, the costs of these services will be borne by the vacating party (individual, department, etc.). 
On occupying new space, personnel are advised to assure themselves that said space is free from hazards. The Environment, Health and Safety Division (Ext. 5251) including the Industrial Hygiene Group (Ext. 5829) can offer advice and assistance. 


\section{G. USING HIGH HAZARD CHEMICALS}

This section of the Chemicai Hygiene and Safety Plan provides information on specific high hazard chemicals or groups of chemicals, and how to control the hazards presented by them. Discussions of the "particularly hazardous" chemicals identified by OSHA (i.e., carcinogens, reproductive toxins, and high acute toxicity substances) are included, along with discussions of other hazardous chemicals that may be pertinent to work conducted at LBL. Solvents, discussed as a group in Section G.7, can be acute toxins and/or chronic toxins. Toxic metals and polychlorinated biphenyls, discussed independently, are generally associated with chronic toxicity rather than acute toxicity. Toxic metals merit a separate discussion because they are encountered in many shop activities. Finally, some of the physical hazards that chemicals can present are addressed (Section G.9).

\section{Definitions and Requirements}

\section{"Particularly Hazardous Substances"}

The OSHA Laboratory Standard dictates that provisions be in place providing additional employee protection for work with "particularly hazardous substances," described as "select carcinogens, reproductive toxins, and substances with a high degree of acute toxicity." Carcinogens and reproductive toxins are discussed in Sections G.2 and G.3. High acute toxicity substances are defined by the Standard as those substances such as hydrogen cyanide, hydrogen sulfide, and nitrogen dioxide which "may be fatal or cause damage to target organs as a result of a single exposure or exposures of short duration."

High acute toxicity substances include substances that are capable of causing intense irritation that can result in pulmonary edema (fluid and swelling in the lungs), chemical asphyxia, and systemic (body-wide) poisoning. As it happens, many substances in this high acute toxicity category are toxic gases, which are discussed in Section G.4. Examples of other substances that are considered to be high acute toxicity substances include:
acrolein
$(\mathrm{PEL}=0.1 \mathrm{ppm})$
acrylonitrile
$(\mathrm{PEL}=2 \mathrm{ppm})$ 


$\begin{array}{ll}\text { 2-aminopyridine } & (P E L=0.5 \mathrm{ppm}) \\ \text { dimethyl sulfate } & (P E L=0.1 \mathrm{ppm}) \\ 1,1 \text {-dimethylhydrazine } & (P E L=0.5 \mathrm{ppm}) \\ \text { hydrogen fluoride } & (P E L=3 \mathrm{ppm})\end{array}$

Note that chemicals known to cause high acute toxicity generally have very low occupational exposure limits (i.e., PELs less than $10 \mathrm{ppm}$ ).

\section{Required Considerations}

The Laboratory Standard allows flexibility in assessing the hazards presented in using particularly hazardous substances and choosing the control measures that best mitigate those hazards. The only baseline requirement for working with particularly hazardous substances is that consideration be given to the following provisions and that they be utilized when appropriate:

(1) establishment of a designated area;

(2) use of containment devices;

(3) establishment of contaminated waste removal procedures; and

(4) establishment of decontamination procedures.

Some of the OSHA substance-specific standards require the use of "regulated areas" to control access and control hazards to particularly hazardous substances. The requirements specified in the substance-specific standards are applicable to nonlaboratory work such as that conducted in LBL shops. The Industrial Hygiene Group assesses the applicability of using regulated areas (and other specific procedures identified in the OSHA substance-specific standards) for LBL's shop operations.

The "designated area" cited in the Laboratory Standard (and therefore strictly applicable to all laboratory work) differs from a regulated area in that the only duty associated with it is to post the area and assure that all employees working in the area are informed of the hazardous substances used there. The designated area may be a room, a section of a room, or actually be a containment device such as a lab hood. The use of designated areas is also discussed in Section F.2, "Administrative Controls." 
Fume hoods or equivalent containment devices are required to be considered for handling "select carcinogens," reproductive toxins, and substances which have a high degree of acute toxicity. Circumstances that may require the use of containment devices when using particularly hazardous chemicals include procedures where (i) the chemicals are volatile, (2) aerosol generation is possible, and (3) manipulations or chemical reactions could result in uncontrolled releases. In addition, if employees experience health effects or if the results of exposure monitoring establish that significant employee exposures are possible, a containment device is required. [See "Hazard Control Measures" (Section F) for a more information on the use of hoods; see "Exposure Monitoring," (Section I) for a discussion on hazard assessment and criteria for monitoring.]

Waste handling procedures for particularly hazardous substances are almost always appropriate and should be addressed in the Specific Safety Procedure (discussed in Section C).

Specific decontamination procedures may or may not be required. Decontamination is more than spill cleanup; decontamination may be required even when no known spill or release has occurred. For example, decontamination procedures are warranted for the use of any carcinogen that could leave residual powders, dusts, or films. Decontamination may not be necessary for highly volatile substances (such as methylene chloride) when used in a hood because residual materials are unlikely.

\section{Chemical Carcinogens}

This section discusses carcinogens and how they are evaluated, and describes the LBL Carcinogen Program. The Carcinogen Program sets forth recommendations and requirements to govern the use of chemical carcinogens for the purpose of minimizing, to the extent practical, the health risks attributed to them.

All LBL personnel using chemical carcinogens are expected to be familiar with the Program guidelines and to conduct their operations accordingly. The purpose of the guidelines is to assist the Principal Investigator or Lab/Shop Supervisor in the selection and use of appropiate safeguards. These safeguards consist of proper engineering controls and safe work practices that permit the safe use of high hazard 
chemicals and maintain exposures to these substances as low as reasonably achievable.

\section{Carcinogen Classification}

Carcinogens are diverse agents that are defined by their ability to cause neoplasms (tumors) in humans and/or animals. Carcinogenic agents may be organic chemicals, inorganic chemicals, hormones, or ionizing radiation. Some carcinogens react directly with a cell's genetic information (the DNA), causing changes (mutations) that are incorporated into subsequent generations of that cell. Other carcinogens do not bind with the DNA, but produce neoplasms after another carcinogen has reacted with the DNA or by some other independent mechanism. For regulatory purposes, no distinction is made for the mechanism by which a chemical is carcinogenic; the key parameter is the strength of evidence for carcinogenicity in humans.

Despite public perception and the apparent large number of chemicals included as known, possible, or suspect carcinogens, not all chemicals are carcinogens. Cancer is caused by distinct mechanisms whereby small, repeated exposures can result in cumulative effects. Consequently, cancer does not manifest itself until a certain latency period has passed, sometimes ten to twenty years after the initial exposure. For this reason it is best to minimize all exposures to carcinogens.

Four organizations /agencies that evaluate and classify carcinogens are (1) the Occupational Safety and Health Administration (OSHA), (2) the International Agency for Research on Cancer (IARC), (3) the National Toxicology Program (NTP), and (4) the American Conference of Governmental Industrial Hygienists (ACGIH).

OSHA regulates health and safety in workplaces. Permissible Exposure Limits (PELs) are established for airborne concentrations of substances to indicate the amounts of a substance to which most workers can be exposed daily for a working lifetime without experiencing adverse effects. Over 400 PELs have been established but they are not generally set to protect workers from potential carcinogenic effects. OSHA does regulate a small number of carcinogens in independent substance-specific health standards (e.g., benzene, asbestos, and vinyl chloride). 
The IARC is a program designed to evaluate existing evidence on chemicals for their carcinogenic risks to humans. IARC places chemicals into four groups: Group 1- carcinogenic to humans; Group 2A - "probably carcinogenic to humans"; Group 2B - "possibly carcinogenic to humans"; Group 3 - not (yet) classifiable as to carcinogenicity; Group 4- probably not carcinogenic.

The NTP is involved with testing and classifying chemicals. A weight-ofevidence approach is used in placing carcinogens into Group 1 , "chemicals known to be carcinogens," or Group 2, "chemicals reasonably expected to be carcinogens."

The ACGIH provides guidelines for occupational exposures, called Threshold Limit Values (TLVs), which are intended to protect workers from harm. The ACGIH recommends TLVs for approximately 55 suspected human carcinogens, based on either limited epidemiological evidence or demonstration of carcinogenesis in one or more animal species by appropriate methods. ACGIH classifications include: A1 - "confirmed human carcinogens", and A2 "suspected human carcinogens."

\section{Select Carcinogens}

Particularly relevant to LBL are those chemicals that the OSHA Laboratory Standard terms "select carcinogens." A select carcinogen includes any substance that meets one of the following criteria:

- it is regulated by OSHA as a carcinogen;

- it is listed under the category "known to be carcinogens" in the Annual Report on Carcinogens published by the NTP (latest edition); or

- it is listed in Group 1, "carcinogenic to humans," by the IARC (latest edition of monograph).

Select carcinogens are also substances listed either by NTP under the category "reasonably anticipated to be carcinogens," or listed by IARC in Group $2 A$ or $2 B$ if 
they have been shown to cause significant tumor incidence in experimental animals in accordance with any of the following criteria:

- after inhalation exposure of 6 or 7 hours per day, 5 days per week, for a significant portion of a lifetime to dosages of less than $10 \mathrm{mg} / \mathrm{m}^{3}$;

- after repeated skin application of less than $300 \mathrm{mg} / \mathrm{kg}$ body weight per week; or

- after oral dosages of less than $50 \mathrm{mg} / \mathrm{kg}$ of body weight per day.

OSHA decided that it is more effective to allow individual laboratories to make determinations as to whether substances listed as NTP Group 2 and IARC Groups $2 \mathrm{~A}$ and $2 \mathrm{~B}$ meet the above criteria for moderate to high carcinogenic potency. Substances on these lists need not be treated as select carcinogens under the Laboratory Standard if data corresponding to the carcinogenic potency criteria described above do not appear in the IARC or NTP documentation or in other existing literature for these substances. However, it is the responsibility of the employer to determine whether literature exists (or does not exist). And, some NTP Group 2 chemicals are also IARC Group 1 chemicals, so the option to independently evaluate the chemical to determine if it classifies as a select carcinogen is voided.

Since it is not feasible for LBL to track and continuously evaluate all the literature on NTP and IARC Group 2 carcinogens, and especially since the only requirement for use of a select carcinogen is consideration for certain specified control measures, LBL chooses to include all NTP and IARC Group 2 chemicals as select carcinogens.

The select carcinogens are listed in Appendix 9. They are listed by class (i.e., OSHA; IARC Groups 1, 2A, and 2B; and NTP Groups 1 and 2). The ACGIH carcinogens are listed in Appendix 10. Then for the convenience of LBL employees, the select carcinogens are listed in one "composite working" list ( the Composite Working Select Carcinogen List) in Appendix 11.

The Composite Working Select Carcinogen List does not include manufacturing processes (e.g.,steel founding) and medical treatments (e.g., cancer therapeutic regimens) included in the first formal list because they are not applicable to LBL operations and processes. In addition, the actual compounds of interest are listed for 
certain group entries (e.g., "hexavalent chromium compounds") to actually identify those compounds. Finally, the working list includes three ACGIH A2 carcinogens (hexachlorobutadiene, vinyl cyclohexene dioxide, and xylidene) that are not technically select carcinogens. DOE Orders require LBL to consider ACGIH carcinogens and to follow OSHA regulations. Therefore, the working list includes ACGIH carcinogens not already included as select carcinogens.

Particularly noteworthy select carcinogens for operations and procedures conducted at $L B L$ are chloroform, benzene, methylene chloride, acrylamide, aniline and benzidine dyes, formaldehyde, and perchloroethylene.

\section{Program Basics}

The LBL Carcinogen Program consist of ten basic elements or steps.

(1) Identification of all the locations in which select carcinogens are stored and used;

(2) Assessment of the feasibility of substituting for less hazardous substances and for reducing the amount and/or frequency of use;

(3) Completion of a Carcinogen Exposure Assessment Survey Form by lab/shop personnel for all carcinogen use/storage locations to identify carcinogen users and to assess their exposure potential;

(4) Ongoing review by the Industrial Hygiene Group of carcinogen purchases;

(5) Development of written Specific Safety Procedures for every procedure/operation using hazardous chemicals to address the hazards and the intended hazard mitigation methods;

(6) Use of labeled designated areas for carcinogen use, with required training as a prerequisite for access;

(7) Use of a containment device (e.g., hood) if appropriate; 
(8) Use of established safe work practices, including decontamination procedures, if appropriate, and procedures for disposing of generated waste;

(9) Exposure monitoring and medical surveillance, as appropriate.

(10) Routine safety audits and self-assessments to identify and correct potential problems

\section{Responsibilities}

Roles and responsibilities regarding chemical hygiene and safety are discussed in Section B of this Plan, and apply to the use of all hazardous chemicals, including carcinogens. However, the following defines responsibilities specific to the Carcinogen Program.

The first step of the Carcinogen Program is to identify which chemicals are considered carcinogens. The Industrial Hygiene Group will assist lab/shop personnel in identifying the carcinogens in their chemical inventories. Many MSDSs and other sources of information use the terms "animal carcinogen," "experimental carcinogen," "suspect carcinogen," "mutagen," etc. Now that an "official" OSHA list is available, i.e., the Select Carcinogen List (Appendix 9, composited in Appendix 11), it is possible to peruse the chemical inventories of all LBL laboratories and shops, looking for specific chemicals.

The Principle Investigator or Lab/Shop Supervisor then determines if the carcinogens present in his/her areas are actually used and/or needed. [It may happen that a carcinogen identified in a location is never actually used, but only resides in the location on some forgotten shelf.]

The best way to protect the carcinogen user, others, and the environment is to keep the use of carcinogens to a minimum. If smaller amounts are routinely kept and used, the risks of exposure and the likelihood of major incidents are reduced. Further, the costs of waste disposal are reduced. Principle investigators and laboratory/shop supervisors must review their procedures/operations to amounts of toxic chemicals used and the frequency of their use. 
All laboratories and shops must participate in a carcinogen exposure assessment survey. A Carcinogen Exposure Assessment Survey Form (Appendix 12) is to be completed by the Principal Investigator or Laboratory/Shop Supervisor to aid the Industrial Hygiene Group in assessing the potential for on-going and projected exposures to carcinogens. Principle investigators and Lab/Shop Supervisors are to track and submit the names of all personnel using carcinogens.

The Industrial Hygiene Group prioritizes inspections based on exposure survey results. Industrial hygienists evaluate the adequacy of control measures, conduct air monitoring as appropriate, and refer to Health Services the names of all personnel identified as potential candidates for medical surveillance. [Unfortunately, established exposure monitoring protocols and/or specific medical surveillance tests are not available for many of the carcinogens (See Sections I and J).]

In addition, the Industrial Hygiene Group reviews chemical purchase requisitions to identify new carcinogen users whose operations/procedures have not been audited for that purpose. The Group reviews Specific Safety Procedures (Section C) written by for each procedure/operation using hazardous chemicals and determines if the intended control measures are adequate for the particular operation/procedure.

Prior to working with carcinogens, all personnel are required to receive training to learn about the health and safety hazards and to understand the specific handling and emergency procedures pertinent to their lab/shop operations and procedures, all stipulated in the Specific Safety Procedures (SSPs). The written SSPs, which include hazard analyses and specific control measures for using the carcinogens, must be filed with the Facility Notebook (See Appendix 4). All SSPs must be updated regularly to reflect current operations, and must be reviewed no less than annually.

EH\&S inspections and Division self-assessments help ensure that laboratory/shop practices, engineering controls, and personal protective equipment are utilized most effectively to reduce the potential for exposure to that level which is as low as reasonably achievable. 
The Industrial Hygiene Group provides technical guidance to personnel regarding the selection of appropriate laboratory practices and engineering controls. The Group also investigates all reported incidents that resuli in exposure of personnel or the environment to chemical carcinogens and will recommend corrective actions that reduce the potential for recurrence.

\section{Control Measures}

OSHA considers carcinogens in the context of laboratory work as only a subset of other particularly hazardous substances. And, OSHA only focuses on those carcinogens for which there is strong evidence indicating human carcinogenicity, (i.e., "select carcinogens"). Laboratories are allowed flexibility in assessing the need for protective measures and in determining the appropriate precautions needed to effectively control exposures to all particularly hazardous substances, including carcinogens. The only "requirements" in terms of control measures are those already outlined in Section G.1 as "required considerations" when using particularly hazardous substances.

In selecting appropriate safeguards, specific attention must be given to:

- The quantity of the carcinogen used;

- The physical and chemical properties;

- The carcinogenic potency;

- The type of experimental procedures involved;

- The frequency of use;

- The engineering controls available in the work area; and

- The applicable health and safety standards.

Adhere to the following rules and guidelines for controlling carcinogen exposures. 
- Maintain an inventory of all carcinogens including the quantities acquired, dates of acquisition, disposition, and the personnel potentially exposed.

- Keep working quantities of carcinogens to a minimum; do not exceed the amounts required for use in one week. This does not include amounts stored in a designated area or a cabinet that is located within the laboratory.

- Use disposable lab coats when working with large quantities of carcinogens and/or with concentrations in excess of $0.1 \%$. [LBL Storeroom, catalog numbers 8405-63672 (large size) and 8405-63673 (extra large size).]

- Wear safety glasses with shields when handling of transferring carcinogens.

- Adhere to all the standard chemical hygiene practices pertinent for using any hazardous chemical (See Section E).

- Know which carcinogens being used are absorbed through the skin and wear gloves appropriate to the task. Discard used gloves after each use and immediately after any obvious contact with a carcinogen.

- Label all primary and secondary containers and place hazard identification signs on entrances to work or storage areas. To obtain appropriate labels and signs, call the Industrial Hygiene Group, Ext. 5829. (Reference labeling requirements discussed in Section E.9 of this Plan).

- Establish designated areas to restrict carcinogen use storage/areas to authorized personnel. Post the areas. Remember that maintenance and emergency personnel must be advised of the potential problems and hazards before entering these work or storage areas.

- To facilitate decontamination, cover work surfaces with stainless steel or plastic trays, absorbant paper with a moisture-proof lining, or other impervious material. Decontaminate or discard the protective covering materials after the procedure has been completed as a hazardous waste. 
- Conduct aerosol-generating procedures or procedures involving volatile carcinogens in a chemical fume hood, a glove box, or other suitable containment equipment. Examples of aerosol-producing operations are the opening of closed vessels; transfer operations; preparation of mixtures; blending; sonification; open vessel centrifugation; and the application, injection, or inoculation of a carcinogen into experimental animals.

- Capture vapors or aerosols produced by analytical instruments with local exhaust ventilation or ventilation into a chemical fume hood.

- Provide a mechanical exhaust ventilation system that controls the air movement from areas of lower contamination potential to areas of higher contamination potential (e.g., from entry corridors into the laboratory).

- Decontaminate obviously contaminated equipment. This is especially applicable to dusts and powders.

- Transfer carcinogens in tightly closed containers placed within a durable outer container.

- Dissolve finely divided powdered carcinogens, if possible, into a liquid. This reduces the possibility of generating an aerosol.

- Use mixtures that are as dilute as possible.

- Place contaminated materials in a closed, plastic bag within a sealed, primary container. Place the primary container in a durable box before transporting.

- Inactivate carcinogens, if possible, before disposal. However, first consult with EH\&S to determine the technical and legal feasibility of the process.

- When cleaning carcinogens that are dusts, use a wet mop or vacuum cleaner equipped with a high efficiency particulate air (HEPA) filter. Do not dry sweesp or dry mop. 
- Protect vacuum lines, pumps, and equipment with an absorbent liquid trap and a HEPA filter to prevent entry of chemical carcinogens into the system. When working with volatile carcinogens, use a separate vacuum pump placed within or vented to a chemical fume hood. This pump should be labeled for use with carcinogens and the oil discharged as carcinogenic waste when it is changed.

- Determine through the EH\&S Division, in consultation with the area supervisor, if exhaust air from open-faced laboratory-type hoods should be cleaned prior to its release to the environment. Treat exhaust air from glove boxes by filtration and/or adsorption, whichever is appropriate.

- Design and operate exhaust air treatment systems, such as filters or absorbers, in a manner that allows maintenance to be performed and avoids direct contact with the collection medium.

- Design exhaust air discharge to minimize the possible reentry of exhaust contaminants into the supply air intake of any building.

\section{Exposure Monitoring and Medical Services}

Employee exposure monitoring (i.e., air sampling) is required by OSHA for a limited number of carcinogens (See Section I.3). For other carcinogens, the Industrial Hygiene Group will assess the need for employee exposure monitoring. Employees must notify the Industrial Hygiene Group of any suspected exposures, of symptoms potentially affiliated with chemical exposures, and of concerns regarding the adequacy of existing control measures. An industrial hygienist will assess the operation or procedure, conduct air sampling as appropriate (and technically feasible), and refer affected employees to Health Services.

Employees may also report known or suspected exposures directly to Health Services, and Health Services will then alert the Industrial Hygiene Group that an investigation is warranted. Both groups must interact because each can supply information necessary to the other. The Industrial Hygiene Group assesses the exposure levels and Health Services (1) assesses the plausibility of the symptoms being associated with the particular agent(s), and (2) decides on the best medical 
surveillance protocols for the particular exposure. Medical consultations and surveillance, including the requirements for specific carcinogens, are discussed in Section J.

Employees must understand their requirement to report all exposures, suspected symptoms, and incidents to their supervisors, who in turn must report them to the Health Services and the Industrial Hygiene Group.

Employees are encouraged to participate in recommended medical surveillance programs to identify changes in their health status. Women who are pregnant must consult with Health Services before the start of any laboratory or shop activity involving hazardous chemicals, including carcinogens.

\section{Reproductive Toxins}

Reproductive toxins are defined in the Laboratory Standard as they are in the Hazard Communication Standard, as "chemicals that affect reproductive capabilities including chromosomal damage (mutations) and produce effects on fetuses (teratogenesis)." Examples of adverse reproductive health effects include birth defects, spontaneous abortion, fetal developmental damage, and infertility.

Toxic chemicals are only one type of agent known to cause reproductive damage. Fatigue, illness, stress, some medications, alcohol, tobacco, ionizing radiation, viruses, and other agents are all known to cause reproductive damage. It is estimated that about $15 \%$ to $30 \%$ of all pregnancies end in spontaneous abortion and the causes of these spontaneous abortions are largely unknown. Also, the background rate for serious birth defects for all newborns is approximately $2 \%$ to $3 \%$. The actual cause of most birth defects is unknown.

Most industrial chemicals have not been adequately tested for their ability to effect reproduction. No agencies comparable to the NTP or the IARC routinely evaluate and classify reproductive toxins. Unfortunately, the lack of rigorous testing and evaluation has resulted in a void of hazard warning information regarding potential adverse reproductive health effects. Most of the information available on human reproductive toxins comes from testing laboratory animals, but most known human reproductive toxins have exhibited similar effects in animals. Therefore, if 
there are any significant data on a chemical that suggests it may cause human reproductive damage, it is prudent to assume it is a reproductive toxin.

The first trimester is the period of most concern to the developing fetus because this is when the organs and the limbs are being formed. During this period many women are not yet aware that they are pregnant. Control measures must already be in place to protect the woman and her fetus from toxic exposure levels. See Section J.7 for further discussion on LBL's policy respecting fetal protection, medical consultations, etc.

It is important to recognize that women, fetuses, and men are all subject to reproductive toxicity. Examples of agents causing birth defects are glycol ethers, lead, ionizing radiation, and certain drugs. Examples of female reproductive toxins are lead and ionizing radiation, which may cause infertility or alter the normal reproductive cycle. Examples of male reproductive toxins that affect fertility are 2-methoxyethanol (an organic solvent) and dibromocloropropane (a banned pesticide).

A list of reproductive toxins, extracted from the State of California Safe Drinking Water and Toxic Enforcement Act (Proposition 65) list of chemical known to cause reproductive toxicity is provided in Appendix 13. Note, however, that many of the listed chemicals are medicines and other chemotherapeutic agents that cause adverse reproductive effects when consumed orally or when injected into the body. The potential for reproductive toxicity resulting from other routes of exposure common to the laboratory use of chemicals (i.e., inhalation and skin absorption) may be negligible. Laboratories and shops should consider, at a minimum, the following chemicals known to cause reproductive effects when inhaled:

carbon disulfide

dinitrobenzene

ethylene glycol monoethyl ether

ethylene glycol monomethyl ether ethylene oxide

lead and lead compounds

mercury and mercury compounds toluene

The control measures appropriate for the particular operation or procedure being conducted depend on a number of factors, including the volatility of the substance, if the substance can be absorbed through the skin (e.g., glycol ethers), and how much of the substance is being used over a time period. Refer to Section G.1 for required 
control considerations and consult with the Industrial Hygiene Group to choose the appropriate control measures.

\section{Toxic and Pyrophoric Gases}

The use of any hazardous gas must be carefully reviewed by the Principal Investigator in cooperation with the Industrial Hygiene Group (for toxic gases) or the LBL Fire Department (for pyrophoric and flammable gases) to ensure that appropriate and adequate safety systems are in place. The concentration of the toxic or pyrophoric gas is considered in the hazard evaluation. It is the responsibility of Division Directors to ensure that this review has been carried out before any new equipment is used or any new procedure adopted

\section{Scope}

Examples of highly toxic and/or pyrophoric gases are:

\begin{tabular}{|c|c|c|}
\hline 1,3-butadiene & germane & phosgene \\
\hline arsenic pentafluoride & hydrogen cyanide & phosphine \\
\hline arsine & hydrogen fluoride & phosphorus pentafluoride \\
\hline boron trifluoride & hydrogen selenide & phosphorus trichloride \\
\hline bromine pentafluoride & hydrogen sulfide & phosphorus trifluoride \\
\hline bromine trifluoride & iodine pentafluoride & selenium hexafluoride \\
\hline arbonyl fluoride & methyl bromide & silane \\
\hline chlorine & methyl chloride & silicon tetrafluoride \\
\hline chlorine trifluoride & methyl silane & stibine \\
\hline cyanogen & nickel carbonyl & sulfur tetrafluoride \\
\hline janogen chloride & nitric oxide & sulfuryl fluoride \\
\hline diborane & nitrogen dioxide & tellurium hexafluoride \\
\hline chlorosilane & nitrosyl chloride & tungsten hexafluoride \\
\hline uorine & oxygen difluoride & vinyl chloride \\
\hline
\end{tabular}

Note: Because Codes and Regulations frequently change, the above list is not intended to be complete. The Industrial Hygiene Office (Ext. 5829) should be consulted if information is needed on the classification of a specific gas not listed above. 


\section{Preparation}

An approved Operatior..? Safety Procedure (OSP), which includes a safety analysis and describes hazard mit.;ation methods, is required before any highly toxic or pyrophoric gas may be procured and delivered for use. See Section C. Under prescribed conditions, when very small amounts of hazardous gases are used, the Industrial Hygiene Group may waive the need for the OSP (refer to Section C for more details).

The facility in which the highly toxic or pyrophoric gas will be used must be constructed and operated in such a way as to meet the applicable requirements of the Uniform Building Code (UBC) and the Uniform Fire Code (UFC). Division Directors are responsible for ensuring that the total quantity of gases contained in their building comply with the UBC and UFC. In buildings where there are programs operated by several program divisions, it will be necessary for the responsible Division Director to clearly define these responsibilities and to delegate them with sufficient authority to ensure that Laboratory policy will be implemented.

Necessary safety equipment such as fume hoods or gas cabinets, safety showers, eyewash, and fire extinguishers must be present. In addition, the experiment apparatus must be designed and installed with the proper safety controls. Contact the Industrial Hygiene Group and/or the Fire Department for assistance.

The research process tool or apparatus must meet all appropriate requirements of the UBC, UFC, NPFA, Life Safety Code, and the LBL Health \& Safety Manual (PUB3000).

Training in accordance with the OSP must be given to all persons operating the process tool or experimental apparatus. In general, it will be the responsibility of the Area Supervisor or Principal Investigator to provide and document his training. In addition, all persons subject to potential hazard from emergency events (e.g., fire fighters and other emergency crew personnel) must have hazardous materials response training. Personnel in adjacent work areas who do not operate hazardous gas equipment will be trained in appropriate actions to take in the event of an alarm or emergency. 


\section{Purchase and Delivery}

Highly toxic and pyrophoric gases will normally be obtained by purchase requisition, form RL-2350 (Appendix 5). The requester must complete the Hazard Review Box on the form. The completed requisition form is to be sent to the Industrial Hygiene Group for approval and forwarding to the Purchasing Department.

It is important that gas cylinders be returned promptly when they are empty or no longer needed by the user. For certain reactive gases, it is very important to comply with the return date and the special instructions placed upon the cylinder. The user of the highly toxic or pyrophoric gas is responsible for removing the cylinder from the equipment to which it is attached, and for scheduling the return of the empty cylinder.

\section{Key Contacts}

Persons planning to order highly toxic or pyrophoric gases should contact the following for information:

- Industrial Hygiene Group, Building 26, Room 30, Ext. 5829, to obtain detailed information on Operational Safety Procedures, safety data sheets on gases, and general inforniation on where to obtain assistance.

- Purchasing Department, Building 69, Ext. 4556, or Industrial Gases Group, Building 69, Ext. 6220, for availability or product information.

- Plant Engineering Department, Building B90K, Ext. 4171, to obtain information on the Uniform Building Code.

- Fire Department, Building 48, Ext. 6015, to obtain information on the Uniform Fire Code.

- UCB Campus Office of Environment, Health and Safety (642-3078) for complete UCB inealth and safety' regulations on all campus buildings other 
than the Calvin and Donner Laboratories (which are within the jurisdiction of LBL).

- The Toxic Substances Safety Subcommittee of the LBL Safety Review Committee, for information on the installation and operation of research apparatus using hazardous gases.

\section{Toxic Metals}

\section{Beryllium}

Beryllium is used predominantly in three forms: beryllium metal, beryllium oxide, and beryllium-copper alloys. Beryllium-copper alloys may consist of 0.5 to $4 \%$ beryllium, although the most common alloy has about $2 \%$ beryllium. Beryllium may also be alloyed with other metals, such as nickel and cobalt, or be found as a salt, e.g., beryllium fluoride, chloride, nitrate, or sulfate, and as beryllium hydroxide.

Exposure to beryllium and its compounds can damage the skin, eyes, and respiratory system. The soluble beryllium salts, especially the fluoride and sulfate, are skin sensitizers and in high concentrations are also primary skin irritants. If beryllium gets into broken skin, the cut may abscess and not heal properly until the substance is removed. Eye irritations are also common, and splash-burns can cause damage to the cornea. Breathing dust and fumes, however, is the most common cause of beryllium poisoning. The effects of inhaling high levels of beryllium can range form mild inflammation of the nose and throat, a condition that resembles a cold, to a severe pneumonia-like reaction characterized by coughing, difficulty in breathing, pain and tightness in the chest, loss of appetite, and general fatigue. The effects of inhaling low levels of beryllium over an extended period of time may be delayed from a few months to years after the last exposure. Chronic beryllium poisoning in most cases affects the respiratory tract. The onset may be manifested by weakness, loss of weight, shortness of breath, and coughing.

Beryllium dusts or powders constitute a moderate fire hazard. However, any fire involving beryllium is a serious threat to the health of nearby personnel. 
Specific work practices and precautions are required for personnel when working with beryllium compounds.

- Avoid skin contact with beryllium salts or salt solutions, and do not allow metallic beryllium to come in contact with open wounds or abrasions. Wear gloves when handling beryllium or beryllium compounds when loose contamination (dust or chips) is present. There is no danger in ordinary skin contact with beryllium metals, alloys, or fused-ceramic material.

- Conduct all operations involving the generation of airborne beryllium under controlled conditions, and obtain approval of these operations from the Industrial Hygiene Group, Ext. 5829. Operations such as machining, grinding, welding, cutting, drilling, sawing, and milling must be enclosed and the exhaust ventilated through high-efficiency filters. A hazardous concentration of beryllium fumes may be generated when the metal is heated above $650^{\circ} \mathrm{C}$ $\left(1200^{\circ} \mathrm{F}\right)$, or when the oxide is heated above $1540^{\circ} \mathrm{C}$ (2800 $\left.\mathrm{F}\right)$. [The following work on beryllium-copper alloys may be performed without special controls: lathe cutting using a coolant, shearing, forming, hand sawing, hand filing, hand sanding, and soft soldering in a hood.]

- Scrupulously adhere to good housekeeping practices and personal cleanliness.

- Store and transport all beryllium parts in labeled containers.

- Ensure that all beryllium and beryllium-contaminated waste is placed in properly marked containers and picked up by the Waste Management Group, Ext. 5251. 


\section{Cadmium}

The greatest exposure potential is probably from welding or burning cadmiumplated parts and brazing or silver soldering with cadmium-containing rods and wires. The brazing alloys contain 15 to $19 \%$ cadmium. Table G.5-1 indicates the important properties of all the silver solders stocked at LBL.

Table G.5-1. Properties of Silver Solders

\begin{tabular}{|c|c|c|c|c|c|c|c|c|}
\hline \multicolumn{2}{|c|}{ Trade Name } & \multirow[b]{2}{*}{ LBL Cat. \# } & \multirow[b]{2}{*}{$\% \mathrm{Cd}$} & \multicolumn{2}{|c|}{ Melt Pt. } & \multicolumn{2}{|c|}{ Flow Pt. } & \multirow[b]{2}{*}{ Description } \\
\hline Englehard Ind & Handy\&Hardman & & & $\mathrm{O}_{\mathrm{F}}$ & $\infty$ & $\mathrm{O}_{\mathrm{F}}$ & ${ }^{\circ} \mathrm{C}$ & \\
\hline Silvaloy $\# 50$ & Easy-Flo & $3439-28753$ & 18 & 1160 & 625 & 1175 & 635 & Strip, $50 \% \mathrm{Ag}$ \\
\hline Silvaloy \#50 & Easy-Flo & $3439-28756$ & 18 & 1160 & 625 & 1175 & 635 & Wire, $50 \% \mathrm{Ag}$ \\
\hline Silvaloy 503 & Easy-Flo \#3 & $3439-41772$ & 16 & 1170 & 630 & 1270 & 690 & Wire, $50 \% \mathrm{Ag}$ \\
\hline Silvaloy \#15 & Sil-Fos & $3439-28747$ & - & 1185 & 640 & 1300 & 705 & Strip, $15 \% \mathrm{Ag}$ \\
\hline Silvaloy $\# 60$ & Braze 603 & $3439-28572$ & - & 1115 & 600 & 1325 & 720 & Wire, $60 \% \mathrm{Ag}$ \\
\hline Silvaloy Easy & Braze Easy & $3439-28751$ & - & 1240 & 670 & 1325 & 720 & Wire, $65 \% \mathrm{Ag}$ \\
\hline
\end{tabular}

Finely divided cadmium metal and cadmium oxide fumes are highly toxic and must not be inhaled or ingested. A single exposure to high levels of cadmium in air can cause severe lung irritation, which may be fatal. Symptoms usually appear 4 to 10 hours after exposure when cough, labored breathing, and commonly a feeling of constriction or a burning sensation in the chest develop. Generalized flu-like symptoms characterized by shaky chills, sweating, aching in the extremities and back, headache, and dizziness may also develop. Continued exposure to low levels of cadmium in air can result in chronic poisoning characterized by irreversible lung injury and kidney damage. Cadmium is suspected of causing cancer in humans. Symptoms of the cumulative effects of cadmium may appear after exposure has terminated. 
Cadmium metal dust will burn with evolution of a very hazardous brownishyellow fume.

The following work practices and precautions are applicable to specific operations involving cadmium compounds:

- Remove all cadmium from plated parts before welding or burning.

- Substitute cadmium-free silver solder whenever possible.

-When cadmium is melted, temperatures should be kept as low as possible, consistent with the requirement of the operation, to prevent excessive fume generation.

- When indoor work or continuous outdoor work involves the generation of airborne cadmium, enclose the process to the maximum extent practical and provide a good exhaust system that collects and removes the fumes as they are formed. If the work is outdoors and intermittent, a properly fitted fume respirator must be used.

- Separate cadmium-containing and cadmium-plated parts from parts not containing cadmium and mark them appropriately so that accidental exposures resulting from cutting and welding do not occur.

- Use a vacuum pickup or wet mopping to clean up cadmium dust. Do not drysweep or blow.

\section{Mercury}

The most widely used form of mercury at LBL is elemental mercury. Mercury also exists as a salt and as an organic compound. From the standpoint of risk to human health, the most important forms of mercury are elemental mercury vapor and short-chain organo-alkylmercurials such as methylmercury and ethylmercury. 
Mercury can enter the body through the lungs, the skin, and the digestive system. Because mercury vaporizes at room temperature, inhalation of its vapors is the most likely route of entry. Short exposures to high levels of mercury vapor can cause acute poisoning-characterized by tightness and pain in the chest, difficulty in breathing, inflammation of the mouth and gums, fever, and headaches. Acute poisoning, however, is rare. Much more common among workers is chronic poisoning caused by long term exposure to lower levels of mercury. Steady exposure can cause a slow build-up of mercury in the body that can result in illness, personality changes, and eventual disability. Symptoms of chronic poisoning include inflammation of the mouth and gums, weakness, loss of appetite and weight, shaking (particularly in the hands), and irritability.

Adhere to the following work practices when working with mercury:

- Avoid skin contact.

- Keep mercury containers closed when not in use.

- Use plastic or metal catch cans under all mercury apparatus that is likely to break or spill; and make transfers over a catch pan.

- Provide adequate ventilation, especially if mercury is heated above room temperature.

- Use a label on all equipment and vessels containing mercury.

- Store in a protected area in closed, labeled containers, preferably plastic. If a glass bottle is used, place it in a secondary container.

- Clean up mercury spills within a work area immediately because mercury vaporizes slowly at room temperature. If mercury is spilled at room temperature, notify the Hazardous Waste Management Group as soon as possible to obtain clean-up equipment and a mercury vapor survey. If mercury is released at elevated temperatures, evacuate the area immediately and notify the emergency dispatcher at the Fire Department, Ext. 7911. 
- Dispose of mercury by placing in sealed, labeled containers. Send unused mercury to Salvage. If the mercury is being disposed of as waste, call the Waste Management Group at Ext. 5251, for pickup. Do not pour mercury down laboratory drains.

- Drain equipment of mercury prior to sending it to the warehouse for storage. Be sure that the equipment is properly secured and tagged with a mercury label.

\section{Reactive Chemicals}

Unstable (reactive) compounds are solids and liquids that in pure state, or as commercially produced or transported, will vigorously polymerize, decompose, combine, or become self-reactive under conditions of shock, pressure, or temperature. Use of such materials must have prior approval from the Industrial Hygiene Group on a case-by-case basis.

\section{Peroxidizable Compounds}

Isopropyl ether, ethyl ether, dioxane, tetrahydrofuran, and other alkyl ethers form peroxides on exposure to air and light. Because these chemicals are packaged in an air atmosphere, peroxides can form even though the containers have not been opened. The longer the storage period of these chemicals, the greater the amount of dangerous peroxides that may form. Experience has shown that isopropyl ether is by far the worst offender.

These peroxides are highly unstable, explosive chemicals that may detonate if subjected to high temperature, shock, or friction. Concentration by evaporation or distillation of an ether with formed peroxides increases the risk of detonation.

Adhere to the following work practices and precautions when working with peroxidizable compounds:

- Purchase ethers containing an inhibitor when possible.

- Store ethers in cans rather than glass bottles. 
- Store ethers in as cool a location as feasible (but not in refrigerators unless flammable proof).

- Test ethers for peroxide content before any distillation procedure and, call EH\&S if peroxides are detected.

- Place safety shields in front of reaction vessels or distillation apparatus in hoods when using ethers.

- Leave at least $10 \%$ "bottoms" when distilling.

- Do not open any container of uncertain age or condition, particularly when the cap or stopper is tightly stuck.

- Dispose of ether containers one year after purchase, or three months after opening. Do not attempt to physically remove or dispose of containers; let EH\&S personnel handle the containers (Hazardous Waste Management Group, Ext. 5251).

- Label containers of ether with a red colored label and indicate the date of purchase. These labels should be applied by storeroom personnel. The container shall be labeled when received and when it is opened by the Area Supervisor or Principal Investigator.

\section{Other Reactive Compounds}

Other reactive chemicals include combustible liquids or solids (e.g., perchloric acid, certairı metals such as potassium and phosphorus), pressure-sensitive chemicals (e.g., picric acid), and unstable compounds (e.g., sodium azide). The following are all considered reactive chemicals that may require special control measures and preauthorization for use, depending on the quantities, concentrations, and intended use. 


$\begin{array}{ll}\text { copper azide } & \text { phosphorus (white) } \\ \text { lead azide } & \text { picric acid } \\ \text { lithium } & \text { potassium } \\ \text { aluminum hydride } & \text { sodium } \\ \text { perchloric acid } & \text { sodium azide } \\ \text { trimethyl aluminum } & \text { triethyl aluminum }\end{array}$

See also Section C on written safety procedure requirements and Section D.3 on the chemical procurement review process. Combustible liquids are also discussed in the next section on solvents.

\section{Solvents}

This section includes discussions on three types of solvents: (1) flammable and combustible liquids, (2) chlorinated hydrocarbons, and (3) fluorocarbon solvents. However, a solvent may fall into more than one class (e.g., a chlorinated hydrocarbon that is flammable).

\section{Flammable and Combustible Liquids}

Technically, flammable and combustible liquids do not burn. However, under appropriate conditions, they generate sufficient quantities of vapors to form ignitable vapor-air mixtures. As a general rule, the lower the flash point of a liquid, the greater the fire and explosion hazard. (The flash point of a liquid is the minimum temperature at which it gives off sufficient vapor to form an ignitable mixture with the air near its surface or within its containment vessel.)

Flammable and combustible chemicals include liquids (e.g., oils, greases, tars, oil base paints, and lacquers) and flammable gases. Flammable aerosols (e.g., spray cans) are also discussed. (Cryogenic and pressurized flammable gases are discussed in the LBL Health and Safety Manual (Pub. 3000), Chapters 7 and 13, respectively.)

Flammable and combustible liquids are defined and divided into classes by the National Fire Protection Association. The classes are described below. 
- Flammable Liquids (Class I). Liquids having flash points below $1000 \mathrm{~F}\left(37.8^{\circ} \mathrm{C}\right)$ and having vapor pressures not exceeding 40 pounds per square inch (absolute) at $100^{\circ} \mathrm{F}\left(37.8^{\circ} \mathrm{C}\right)$. Flammable Class I liquids are subdivided as follows:

Class IA. Liquids having flash points below $73^{\circ} \mathrm{F}\left(22.8^{\circ} \mathrm{C}\right)$ and boiling points below $100^{\circ} \mathrm{F}\left(37.8^{\circ} \mathrm{C}\right)$. Flammable aerosols (spray cans) are included in Class IA.

Class IB. Liquids having flash points below $73^{\circ} \mathrm{F}\left(22.8^{\circ} \mathrm{C}\right)$ and having boiling points at or above $100^{\circ} \mathrm{F}\left(37.8^{\circ} \mathrm{C}\right)$.

Class IC. Liquids having flash points at or above $73^{\circ} \mathrm{F}\left(37.8^{\circ} \mathrm{C}\right)$ and below $100^{\circ} \mathrm{F}$ $\left(37.8^{\circ} \mathrm{C}\right)$. The boiling point is not considered.

- Combustible Liquids (Classes II and III). Liquids having flash points at or above $100^{\circ} \mathrm{F}\left(37.8^{\circ} \mathrm{C}\right)$. Combustible liquids in Classes II and III are subdivided as follows:

Class II. Liquids having flash points at or above $100^{\circ} \mathrm{F}\left(37.8^{\circ} \mathrm{C}\right)$ and below $140^{\circ} \mathrm{F}$ $\left(60.0^{\circ} \mathrm{C}\right)$.

Class IIIA: Liquids having flash points at or above $140^{\circ} \mathrm{F}\left(60.0^{\circ} \mathrm{C}\right)$ and below $200^{\circ} \mathrm{F}$ $\left(93.4^{\circ} \mathrm{C}\right)$.

Class IIIB: Liquids having flash points at or above $200^{\circ} \mathrm{F}\left(93.4^{\circ} \mathrm{C}\right)$.

Fires involving combustibles and flammables are especially dangerous because they release heat quickly, causing the fire to spread rapidly. The handling and use of these combustibles presents the most significant single source of fire hazard at LBL. Misuse or improper storage threatens not only the researcher and the experiment, but the laboratory unit and the entire building.

Liquids with flash points below room temperature (Class IA and IB liquids) continually emit sufficient quantities of vapors to be ignitable, except when chilled to temperatures below their flash points. Even when chilled, if spilled on a floor or work surface, they will heat rapidly and pose severe fire and explosion hazards. Liquids with flash points above room temperature (Class IC, II, IIIA, and IIIB liquids) 
can easily be heated to the point at which they will create flammable vapor-air mixtures.

Flammable liquid vapors are heavier than air. They can travel for appreciable distances and accumulate in low places. Since it is the vapor of flammable liquids that burns, the fire hazard may not be confined to the immediate vicinity of actual use. Vapors can be ignited several hundred feet from the point of vapor generation. Flammable liquid vapors generally have low ignition-energy requirements and can often be ignited by small sparks from electrical motors, switches, relay contacts, etc.

Recommended precautions are based on the properties of the liquid to be used and the intended application. The user cannot make a correct decision on necessary precaution unless the properties of the liquid are known and the intended use is reviewed from a safety standpoint.

There must be sufficient ventilation to preclude the accumulation of flammable vapors. Flammable liquids should be used in a fume hood or with local exhaust ventilation. Normal room ventilation may be sufficient to permit small-scale use of flammable liquids (milliliter quantities). However, if larger quantities of liquid must be used in such facilities, it will be necessary to provide additional ventilation by opening doors and windows or providing some form of temporary exhaust ventilation.

Extreme care must be exercised when using flammable liquids in closed ("confined") spaces with minimal ventilation (such as glove boxes and tanks). Even milliliter quantities of flammable liquids can cause the build-up of explosive mixtures in the confined space.

Water should not be applied to fires involving flammables or combustibles. The use of water may float burning liquids, causing the fire to spread more rapidly. The fires are usually extinguished by excluding the air around the burning liquid. This is accomplished by one of several approved types of fire extinguishing agents, e.g., carbon dioxide, ABC multipurpose dry chemical, and Halon 1301 (a vaporizing liquid that breaks the flame front).

Many flammable and combustible liquids also pose health hazards. 
It is the responsibility of the user to ensure that all flammables and combustibles are properly identified, labeled, handled, and stored. If assistance is required, contact the Fire Department.

The maximum allowable sizes of containers and portable tanks are provided below.

Table G.7-1. Containers for Combustible \& Flammable Fluids

$$
\text { Flammable liquids Combustible liquids }
$$

$\begin{array}{lllllll}\text { Class } & \text { 1A } & \text { 1B } & \text { 1C } & \text { II } & \text { III }\end{array}$

$\begin{array}{llllll}\text { Glass or approved plastic } & 1 \mathrm{pt}^{\mathrm{a}} & 1 \mathrm{qta}^{\mathrm{a}} & 1 \mathrm{gal} & 1 \mathrm{gal} & 1 \mathrm{gal} \\ \text { Metal (other than Depart- } & 1 \mathrm{gal} & 5 \mathrm{gal} & 5 \mathrm{gal} & 5 \mathrm{gal} & 5 \mathrm{gal}\end{array}$

ment of Transportation

(DOT) drums)

Safety cans ${ }^{b}$

Metal drums

(DOT specifications)

Approved portable tanks 660gal 660gal 660gal 660gal 660gal

a Glass or approved plastic containers or no more than 1 gallon capacity may be used for Class IA or IB flammable liquids if (1) such liquid either would be rendered unfit for its intended use by contact with metal or would excessively corrode a metal container so as to create a leakage hazards or (2) the user's process either would require more than 1 pint of a Class IA liquid or more than 1 quart of a Class IB liquid, of a single assay lot, to be used at one time.

b Approved safety cans of various materials and capabilities are available through the Central Storeroom (LBL stock, Section 7960). 
The following rules and precautions apply to the use and/or storage of flammable/combustible liquids:

- Storage cabinets must be designed and approved for the anticipated usage. Approved metal storage cabinets are available in various sizes from stores (LBL stock, Section 7195).

- Not more than 120 gallons of Class I, Class II, and Class IIIA liquids, combined, may be stored in a storage cabinet. Of this total, not more than 60 gallons may be of Class I and Class II liquids, combined, and not more than three such cabinets may be located in a single fire-separation area.

- Ordinary domestic refrigerators must not be used for the storage of flammable liquids because they contain certain built-in ignition sources (such as electrical contacts). These sources of ignition may initiate a fire or an explosion if flammable vapors are present. In special cases, ordinary refrigerators have been modified by the Electricians' Shop to specifications approved for storage of flammable liquids. Refrigerators are now available commercially that are specifically designed and approved for storage of flammable materials.

- To adequately manage the exposure hazards in each building or fire-separation area in each building, it is necessary to consider the needs of all users, and/or of user groups in aggregate, for each building or fire-separation area.

Quantity restrictions are usually intended to provide guidance for lower usage levels. In general, quantities in excess of three-months usage should not be stored. If the need for larger quantities is anticipated, contact the Fire Department for assistance. The maximum allowable quantities for flammables and combustibles outside designated and approved storage rooms or facilities are listed below:

- One gallon size is the maximum allowable container size for for general dispensing of Class I and Class II liquids unless in approved safety cans.

- The maximum allowable safety container size for Class I-A flammable liquids is 2 gallons. 
- The maximum allowable safety container size for Class I-B, Class I-C, Class II, and Class III-A liquids is 5 gallons.

- Where more than one laboratory unit or shop is located in a single fireseparation area, all Class I and Class II liquids must be stored in approved storage cabinets or approved safety cans.

- For single fire-separation areas, 10 gallons of flammable and combustible liquids, combined, is the maximum quantity allowable outside of approved storage cabinets or approved safety cans.

\section{Chlorinated Hydrocarbons}

The chlorinated hydrocarbons as a whole have many industrial as well as laboratory uses. At LBL they are commonly used as cleaners, degreasers, paint removers, solvents, and extractants.

Most of these compounds have an anesthetic (narcotic) effect, causing workers to feel "drunk," become unconscious, or even die if the amount of inhaled vapor is excessive. Individuals working around moving machinery can be subject to accidents when their judgment and coordination are impaired by the anesthetic effects of inhaled solvents. Usually it is the anesthetic effect that is responsible for sudden unconsciousness of persons exposed to solvents in tanks, pits, and other confined spaces. Trichloroethylene, ethylene dichloride, and chloroform are examples of compounds that are powerful anesthetics.

Some, but not all, of the chlorinated hydrocarbons are strong poisons that damage the liver, kidneys, nervous system, and/or other parts of the body. This damage may be permanent or even cause death, although recovery from lesser exposures does occur. Single exposures to higher concentrations of vapors, as well as repeated exposure to small concentrations can produce symptoms of poisoning. These symptoms most often come on gradually, with nausea, loss of appetite, vomiting, headaches, weakness, and mental confusion most often noted. Carbon tetrachloride, tetrachloroethane, and 1,1,2- trichloroethane are examples of compounds that are strong poisons. 
All chlorinated hydrocarbons on repeated contact with the skin can cause rashes (dermatitis) because of their ability to remove the protective fats and oils from the skin. A few of these solvents are known to be capable of entering the body through contact with the skin. In addition, many of these compounds are highly irritating to the membranes around the eyes and in the nose, throat, and lungs. Examples of chlorinated hydrocarbons that have irritant properties are ethylene dichloride and chloroform.

Many of these compounds are on the Select Carcinogen List, e.g.,carbon tetrachloride, methylene chloride, chloroform, perchloroethylene, trichloroethylene, and ethylene dichloride. See Section G.2 for further discussion on carcinogens and Appendix 10 for lists of select carcinogens.

When heated, these compounds can decompose, forming highly toxic fumes of phosgene, hydrochloric acid, and chlorine. Most of the chlorinated hydrocarbons are nonflammable; however, there are exceptions. Table G.7-2 lists important characteristics of some of the common chlorinated hydrocarbon solvents. For questions concerning the hazards of a specific compound, contact the Industrial Hygiene Group, Ext. 5829. 
Table G.7-2. Chlorinated Hydrocarbon Data

\begin{tabular}{|c|c|c|c|c|}
\hline Common Name & Chemical Name & $\begin{array}{l}\mathrm{PEL}^{\mathrm{a}} \\
(\mathrm{ppm})\end{array}$ & $\begin{array}{c}\text { Volatility } \\
(\mathrm{mm} \mathrm{Hg})\end{array}$ & Flammability \\
\hline Acetylene dichloride & 1,2-Dichloroethylene & 200 & 200 & Moderate \\
\hline Carbon tetrachloridec & Tetrachloromethane & 2 & 115 & Nonflammable \\
\hline Chloroform $\mathrm{c}$ & Trichloromethane & 2 & 200 & Nonflammable \\
\hline Ethylene dichloride ${ }^{c}$ & 1,2-Dichloroethane & 1 & 80 & Moderate \\
\hline Methyl chloroform & 1,1,1-trichloroethane & 350 & 132 & Nonflammable \\
\hline Methylene chloride $c$ & Dichloromethane & $25^{*}$ & 435 & Nonflammable \\
\hline Perchloroethylene ${ }^{c}$ & Tetrachloroethylene & 25 & 18 & Nonflammable \\
\hline Tetrachloroethane & 1,1,2,1-tetrachloroethane & 1 & 8 & Nonflammable \\
\hline Trichloroethane & 1,1,2-trichloroethane & 10 & 25 & Nonflammable \\
\hline Trichloroethylene & Trichloroethylene & 50 & 76 & Nonflammable \\
\hline \multicolumn{5}{|c|}{$\begin{array}{l}\text { The Permissible Exposure Limit (PEL) is expressed as parts of pure solvent vapors per million } \\
\text { parts of air (ppm). The asterisk }(*) \text { by the methylene chloride PEL signifies that this is the } \\
\text { value in the new proposed rule (Nov., 1991). }\end{array}$} \\
\hline \multicolumn{5}{|c|}{$\mathrm{b}$ The vapor pressure at $77^{\circ} \mathrm{F}\left(25^{\circ} \mathrm{C}\right)$} \\
\hline
\end{tabular}


Adhere to the following rules and precautions when using chlorinated hydrocarbons.

- Consider information on the occupational exposure limits (i.e., the OSHA PEL and the ACGIH TLV), the volatility, and the flammability of a compound. These three characteristics always must be taken into careful consideration in selecting a compound in order to minimize the health hazards connected with its use.

- If there is a possibility of skin or eye contact, wear the appropriate protective equipment. Gloves made of impervious material should be worn for hand protection. Reference Appendix 6 or call the Industrial Hygiene Group (Ext. 5829) for recommendations on the types of materials that are acceptable.

- For high vapor concentrations (and especially if the solvent is on the Select Carcinogen List), control exposures by using local exhaust ventilation. If the solvent is on the select carcinogen list, consider the need for local exhaust ventilation even for moderate and low vapor concentrations. Contact the Industrial Hygiene Group, Ext. 5829, for an assessment of the airborne concentration.

- Store chlorinated hydrocarbons in cool, dry, and well-ventilated areas. Containers should be checked for leaks because metal corrosion can occur from hydrochloric acid produced by the decomposition of the solvent.

Decomposition may occur under conditions of high temperature, exposure to moisture, and exposure to ultraviolet light.

- Label compounds, both in the original containers and in secondary containers used by employees, so that the potentially injurious substances are plainly identified. Labels for perchloroethylene, trichloroethylene, and carbon tetrachloride can be obtained at the LBL Central Storeroom, Bldg. 7.

- Place waste chlorinated hydrocarbons in an organic liquid waste can for disposal. When the waste can is full, contact the Waste Management Group. 


\section{Fluorocarbon Solvents}

Fluorocarbon solvents are organic compounds containing fluorine. Many also contain chlorine and are technically "chlorofluorocarbons." Common names for some members of this family are Freon-TF, Freon-TMC, and Freon-11.

The vapors are four to five times heavier than air and tend to accumulate in tanks, pits, and low places. This displaces the oxygen, which can cause suffocation, or the vapors themselves may be toxic in high concentrations.

Fluorocarbon solvent will dissolve and extract the natural oils present in the skin. If contact is prolonged, the skin may become dry and perhaps cracked. The vapors have little or no effect on the eyes. If the liquid is splashed in the eyes, temporary redness may be produced.

Lower boiling liquids may cause freezing if splashed on the skin or in the eyes.

Fluorocarbon vapors decompose when exposed to high temperatures, ultraviolet and radiofrequency radiation. Toxic fumes such as hydrofluoric acid, hydrochloric acid, and phosgene may be formed. Fluorocarbon solvents are nonflammable.

Adhere to the following rules for work with fluorocarbons:

- Contact the Industrial Hygiene Group, Ext. 5829, if fluorocarbon solvents are used in enclosed areas such as tanks and pits. A confined space entry permit is required, and forced-air ventilation and air-supplied respirators may be required.

- Avoid contact with hot surfaces, electric heating elements, or open flames. If toxic fumes are formed, good ventilation will be required.

- Wear gloves made of neoprene or equivalent when there is the possibility of prolonged or repeated skin contact with the liquid. Wear protective clothing and eye goggles if the liquid may be splashed. 
- Control environmental releases pursuant to air quality regulations (to protect the earth's ozone layer).

\section{Polychlorinated Biphenyls (PCBs)}

PCBs are a broad class of nonflammable, synthetic, chlorinated hydrocarbon insulating fluids previously used in capacitors and transformers. Synonyms include askarel, aroclor, inerteen, pyranol, therminol, and many others.

Prolonged skin contact with PCB oils can cause skin irritation and occasionally the formation of acne-like cysts. Eye contact can cause severe irritation and inflammation. Breathing the vapor or mist from heated oil can cause respiratory irritation. PCBs are listed as suspect carcinogens and can cause liver damage.

PCBs may produce toxic and carcinogenic dioxin compounds when heated, as in a fire. But generally, PCBs do not break down in the environment, due to their inert character and stability under extreme physical stresses. PCBs are widely dispersed in the environment and can accumulate in foods found in the human diet.

The following precautions apply when working with PCBs:

- Wear the appropriate protective equipment including viton gloves, coveralls, and splash goggles when working with PCB-contaminated equipment or on PCB spills.

- Clean up small PCB spills with vermiculite or Sorb-all. Place waste material in plastic bags and call the Waste Management Group, Ext. 5251. All equipment containing PCBs must be disposed of through the EH\&S Waste Management Group.

- In case of large PCB spills or explosions of PCB-containing equipment, evacuate all personnel from the area. Call the Fire Department, Ext. 7911, for assistance. Provide or maintain ventilation in the affected area, if possible. If entry to the area is necessary, a self-contained breathing apparatus must be worn. 
- If PCB splashes on an employee, remove any contaminated clothing promptly and wash the skin with soap and water. If the eyes are affected, flush immediately for about 15 minutes. Report to Health Services.

- Place equipment containing PCBs in a manner to ensure minimum contamination to the environment. Equipment must be stored and transported in a manner that eliminates any leaks to the environment.

- Label all equipment containing PCBs. Note: PCBs have been phased out of all the large utility transformers at LBL.

\section{Physical Hazards}

\section{Compressed Gases}

The general precautions for compressed gas cylinders must be followed. [See the LBL Health and Safety Manual (Pub-3000), Chapter 13]. The basic rules are summarized below:

- Do not purchase large cylinders of hazardous gases if it is possible to use small cylinders.

- Do not use cylinder color coding to identify cylinder contents. These colors have not been standardized by the suppliers. Rely on the label placed on the cylinder.

- Return empty cylinders to the vendor as soon as possible after use. It is not uncommon for gas cylinders to develop leaks during storage. Arrangements for pickup of used cylinders are made by contacting LBL Transportation. Before pickup, the cylinder valve must be closed, the regulator or needle valve must be removed, the dust cap replaced, and the valve cover put back on the cylinder.

- If a cylinder is leaking, contact the Industrial Hygiene Group or the Fire Department. 


\section{Oxygen-Pumping in Vacuum Systems}

Oxygen in concentrations $25 \%$ by volume should not be introduced into a mechanical vacuum pump charged with hydrocarbon oil, which is a combustible fluid. During compression in the pump, the pressure of the oxygen may reach as high as 2-3 atmospheres, and at this pressure it may cause an explosion if combined with a hydrocarbon oil.

Various fluids are available, such as Fomblin or Halo Vac (Sargent-Welch Scientific Company). Modification of the pump may be required because these fluids have high molecular weights and high specific gravities and may be incompatible with seals. An inquiry to the pump manufacturer is recommended.

Pumps modified for oxygen service shall be permanently identified and used only with the specified fluid.

\section{Cryogenic Hazards}

The primary hazard of cryogenic materials is extreme coldness. They, and surfaces they cool, can cause severe burns if allowed to contact the skin. Division guidelines and standards for the safe storage and handling of cryogenic materials are given in the LBL Health and Safety Manual (Pub-3000), Chapter 7, however, the following are some general rules for laboratory operations:

- Use gloves and a face shield when preparing or using cold baths.

- Do not use liquid nitrogen or liquid air to cool a flammable mixture in the presence of air because oxygen can condense frorn the air, and can lead to an explosion.

- Use appropriate dry gloves and face shields when handling dry ice, and add the ice slowly to the liquid portion of the cooling bath to avoid foaming. Workers should avoid lowering their heads into a dry ice chest to prevent suffocation.

- Transport materials packed in dry ice in such a manner as to prevent the accumulation of carbon dioxide gas. 


\section{H. TRAINING AND EMPLOYEE INFORMATION}

\section{Requirements}

Employees must be provided training on the hazards to which they may be exposed and the means to avoid these hazards. Training must be updated when a new hazard is introduced into the workplace.

Safety training is the responsibility of the employee's division or department. New Employee Safety Orientation is required for all new employees and participating guests within the first month of work. To assist the departments, other safety training is conducted by EH\&S (See Section H.3). However, the division or department is responsible for providing specific job safety orientation, hazard communication, chemical hygiene and safety, and skills training. Supervisors must instruct their personnel about the potential hazards involved in their work, the proper safety precautions to follow, the warning signs of possible exposures to chemicals in their operations (e.g., odors, irritation, etc.), and the emergency procedures to use if an accident should occur.

Complete training for those working with hazardous chemicals involves three levels of training:

(1) The LBL New Employee Safety Orientation (Course EHS-10), which provides general hazard communication training,

*(2) CHSP Training (Course EHS-348), a course geared toward the elements of this Plan, and

(3) Operation/procedure-specific training provided individually or in small groups by the Principal Investigator or Laboratory/Shop Supervisor.

* The Chemical Hygiene and Safety Plan training provided by EHES ("CHSP Training") consists of two different formats: one geared towards laboratory operations, and one geared towards shop operations. 
Collectively, the training must minimally include discussion of the following topics:

- The requirements of the Hazard Communication and Laboratory Standards, whichever applies

- Potential chemical, physical, and biological hazards

- Which operations involve hazardous materials

- Applicable health standards (e.g., OSHA PELs and ACGIH TLVs)

- Use and location of Material Safety Data Sheets

- Labeling requirements

- General safe working practices

- Purpose and use of control measures

- The use of administrative controls (e.g., controlled areas, designated/regulated areas, etc).

- Personal protection measures

- The warning properties of chemical releases (e.g., odors, eye irritation, etc.)

- The signs and symptoms of chemical overexposure

- Purpose and results of exposure monitoring

- Purpose and requirements of medical surveillance

- Spill response and emergency protocols

- Possible non-routine tasks 
- Hazards of unlabeled pipes, wastes, etc.

A Safety Training Check List, attached as Appendix 14, lists specific training topics.

\section{Hazard Communication Resources}

Information on hazardous properties of a chemical substance can be accessed through the following resources:

\section{Material Safety Data Sheets (MSDSs)}

Manufacturers and distributors are required to develop a material safety data sheet (MSDS) for each hazardous material they produce or import. All MSDSs provide information regarding the specific chemical identity of the material(s) involved and their common names; information on its physical and chemical characteristics; known acute and chronic health effects and related health information; exposure limits; precautionary measures; emergency and first aid procedures; and the identification of the organization responsible for preparing the sheet. Refer to Appendix 15 for details on "Using the Material Safety Data Sheet."

Every work area at LBL, with the exception of individual research laboratories, must have readily accessible to the employees an MSDS for every hazardous material used in the area. The Principal Investigators and Lab/Shop Supervisors are responsible for securing MSDSs and making sure that they are available (See Section B.2). If an MSDS is inadvertently not received at the time of the first shipment, a copy may be obtained. through the files in the Industrial Hygiene Office (Ext. 5829). If the MSDS is not on file, the Industrial Hygiene Group will contact the manufacturer and obtain one.

Individual laboratories and shop areas must maintain those MSDSs that are received with their shipments. In some cases an MSDS is not needed for all chemical products. For example, when a laboratory or shop purchases a household consumer product that contains a hazardous substance (e.g., Formula 409 allpurpose cleaner), and the product is used in a household application similar to that intended by the manufacturer, a MSDS is not required. 


\section{Computer MSDSs}

Two additional resources are available to LBL laboratory personnel as "on-line" MSDS Systems: Canadian MSDSs, and some manufacturer-specific MSDSs available from the UC Office of the President. Both are accessed via LBL Ethernet. Refer to Appendix 15 for an example of a Chernical Infogram (MSDS) that can be accessed through the Ethernet System.

\section{TOMES}

The Micromedex TOMES (Toxicology, Occupational Medicine, and Environmental Series) System is a combination of a number of excellent databases on chemical hazard information. Included on one compact disk, and updated every 90 days, the information (685 megabytes, equivalent to a stack of references 50 feet high) includes toxicity data, hazardous reactions, ernergency response guidance, environmental regulations, fire safety, etc.

\section{EMS}

The Environmental Management System (EMS) is the database that holds LBL's Chemical Inventory files. The locations of every chemical, site-wide, can be determined as well as the room-by-room inventory listings.

\section{Books}

Refer to Section $L$ for a list of recommended references and information resources.

\section{Training Resources}

A listing of EH\&S courses is published in the quarterly Health and Safety Course announcement. Supervisors or others planning safety meetings or training may obtain educational materials, audiovisual aids, and advice from EH\&S. 
One course specifically designed for laboratory and shop supervisors is strongly recommended and may be required. The course presents an overview of EH\&S requirements/responsibilities:

- EH\&S Training for Supervisors

Other courses that may be required or recommended are dependent on the operations, chemical agents and/or physical hazards:

- Chemical Safety for Semiconductor Operations

- Radiation Safety Orientation

- Radiation Protection - Radionuclides

- Respirator Use

- Laser Safety

- Forklift Safety

- Incidental Crane Operator Safety

- Hearing Conservation

- Confined Space Entry

- CPR

- First Aid

- Fire Extinguisher Use

- Waste Generator's Training

- Pressure Safety/Compressed Gases 


\section{EXPOSURE MONITORING}

\section{Purpose}

Exposure monitoring refers to testing the air for the presence of specific chemicals or types of chemicals. Measurements are of two types, direct and time-integrated. Direct measurements provide information on the air concentration of a chemical at one moment in time and are useful for screening purposes. Time-integrated sampling allows the industrial hygienist to establish the average air concentration of the chemical over a specific period of exposure. Results of time-integrated exposure monitoring can be compared to legal exposure limits (i.e., PELs).

The purpose of exposure monitoring is to:

(1) Identify laboratory or shop operations/procedures requiring improved controls;

(2) Establish the effectiveness of existing controls for new or altered operations/procedures;

(3) Identify the personnel potentially or actually exposed to hazardous chemicals at unsafe levels, including those personnel who report symptoms consistent with exposure; and

(4) Establish safe air concentrations following cleanup of chemical spills/releases.

The Industrial Hygiene Group must measure an employee's exposure to any substance regulated by an OSHA standard (which includes any chemical with an OSHA PEL) if there is reason to believe that exposure levels for that substance exceed the action level (or in the absence of an action level, the PEL). The initial monitoring must be followed by periodic monitoring if the results of the initial monitoring indicate exposures at or above the action level or PEL. (See Section I.4 or Appendix 3 definitions on PELs, action levels, etc.) 
Employee exposure monitoring is sometimes used during an initial evaluation of an operation to determine if controls are needed. This is performed on a test basis during the initial phase of an operation. Exposure monitoring may also be performed, if needed, after the operation has been implemented to verify that exposure levels are safe. Results from the employee exposure monitoring are used to determine whether any additional controls are needed.

\section{Hazard Assessment and Criteria for Monitoring}

Generally, exposure monitoring is just one part of the hazard assessment process, and it is frequently not warranted. The first steps in a hazard assessment of any laboratory or shop operation/procedure include an evaluation of the following factors:

- The physical and chemical properties of the chemical (e.g., volatility, reactivity);

- The toxicity of the chemical, i.c., the acute and chronic health effects attributed to specific exposure levels, including low, long-term, and brief high exposure levels; and

- The "routes of exposure" or ways that the chemical can enter the body, such as via inhalation and/or skin absorption.

A hazard assessment is conducted for each laboratory or shop procedure as part of the Laboratory/Shop Specific Safety Procedure (SSP). See Section C and Appendix 4.

The decision to conduct exposure monitoring of an operation or procedure is based upon consideration of the potential amount of exposure. Factors to consider in assessing the need or benefit of exposure monitoring include:

- How long the operation/procedure lasts. Monitoring is usually not needed if the exposure lasts fewer than 10 minutes and involves materials of low toxicity/hazard. 
- The presence of other controls (e.g., if the operation/procedure is performed in a lab hood and the hood is functioning properly, monitoring is usually not needed).

- The number of employees involved. Monitoring has greater priority if many employees are being exposed.

- The physical form of the substance. Airborne emissions are unlikely from solid metal bars but possible with metal powders.

- The amount of substance being used or released. If only small quantities (e.g., 10 milliliters or 1 gram, or low concentrations) of the material are being used, and/or if materials are in closed containers, monitoring may not be needed. However, if visible emissions are present (e.g., visible dust, fumes) or if vapors are released over a significant time period (e.g., 30 minutes or more), monitoring may be needed.

- Availability of established monitoring methods. Sampling and analytic methods are not available for all chemicals.

\section{Substances that Require Monitoring}

Employee exposure monitoring is not required for laboratories unless there is reason to suspect exposures are at or above (1) the OSHA Action Level (which for chemicals that have them, is usually one-half of the PEL value), or (2) the OSHA PEL, for chemicals that do not have Action Levels. However, certain substancespecific OSHA standards (e.g., the lead standard) do require monitoring to establish exposure levels, and specify monitoring protocols. LBL shops are subject to the monitoring requirements of these substance-specific standards. The Industrial Hygiene Group determines the appropriate exposure monitoring needs for the shops and laboratories.

The list of substances with specific monitoring requirements is given below:

acrylonitrile

asbestos 


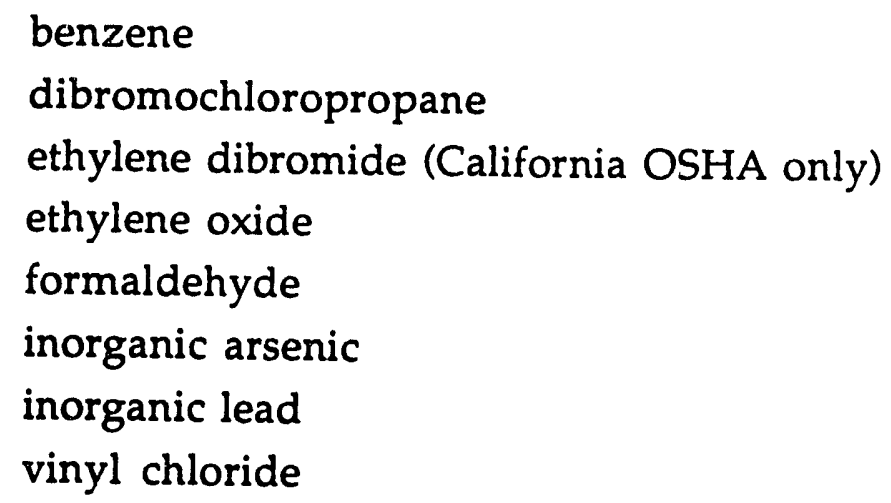

\section{Interpretation of Results}

Monitoring is performed under the direction and management of an industrial hygienist and the monitoring results are compared with accepted criteria:

- The OSHA Permissible Exposure Limit (PEL) for a substance. The PEL is a legal limit that must be met for any operation, laboratories included. The PEL represents an 8-hour, time-weighted average concentration.

- The American Conference of Governmental Industrial Hygienists (ACGIH) Threshold Limit Value (TLV). A TLV is a recommended limit that should be used as a guideline. The TLV is also an 8-hour, time-weighted average concentration.

- The OSHA Action Level (AL) for the substance, if one exists. An action level, usually one-half the PEL, triggers additional monitoring and/or medical surveillance requirements. The AL is measured for a workday i.e., an 8-hour time-weighted average concentration.

- The OSHA and ACGIH Short-Term Exposure Limit (STEL) is the average concentration to which workers can be exposed for a short period of time (15 minutes). The STEL supplements the 8-hour PEL/TLV and is intended to protect workers from acute toxic effects.

Many substances have both a PEL and a TLV. In some cases, the values of these two limits are different for the same substance. In these cases it is LBL's policy, pursuant to DOE orders, to use the lower of the two limits to evaluate the exposure results. 
LBL's Industrial Hygiene Group shall within 15 working days after the receipt of any monitoring results, notify the employee's supervisor. The supervisor will in turn (and within the same 15-day period) notify the affected employee(s) of the results. The notification must be in writing and either presented individually or by posting the results in an appropriate location that is accessible to the employee(s). Consideration is given to an employee's desire for confidentiality.

The Industrial Hygiene Group shall notify Health Services of potential exposure problems by providing them (1) a copy of the completed Hazard Evaluation Form, which is routinely used by the Group (See Appendix 12), and (2) a copy of the memo relaying exposure monitoring results to the employee and his/her supervisor. The Hazard Evaluation Form is also used to document follow-up evaluations that occur after Health Services refers potential exposure problems to the industrial Hygiene Group (See Section Section J.5). 


\section{J. MEDICAL CONSULTATIONS AND SURVEILLANCE}

\section{Overview of Medical Services}

Medical consultation and examinations are provided under the direction of Health Services, a group within the EH\&S Health Department. The mission of Health Services is to provide a comprehensive occupational health program. The primary objectives of the program are to:

- Ensure that employees are assigned duties they are physically able to perform;

- Provide medical care and rehabilitation of the occupationally ill and injured;

- Provide emergency treatment for illnesses and injuries;

- Encourage employees to maintain their physical and mental health;

- Assist in maintaining a healthful and safe work environment; and

- Monitor for signs and symptoms of possible exposure or overexposure to hazardous chemicals, infectious agents, noise, and radiation.

Health Services provides emergency medical services, medical consultations, and medical examinations. A medical consultation is a consultation that takes place with a health care provider for the purpose of determining whether a medical examination and/or medical procedures are appropriate. A medical examination is a physical examination by a licensed physician for the purpose of assessing health status. Medical examinations are one of three types: pre-placement, periodic (e.g., for medical surveillance), and employment termination.

A medical examination usually consists of an occupational and medical history and tests to identify health problems. Special attention is given to potential health effects that are associated as health hazards specific to hazardous agents that an employee may be exposed to in the work area. Potential occupational exposures to hazardous situations or agents are investigated on a continuing basis in 
cooperation with Industrial Hygiene, Occupational Safety, and other groups in the Environment, Health \& Safety (EH\&S) Division.

Medical surveillance is the application of clinical procedures ("medical screening") to select personnel who are identified as being at risk for exposure to hazardous agents at occupationaliy significant levels. Clinical procedures may be utilized to detect evidence of the agent itself (e.g., lead in the blood), or clinical manifestations resulting from the agent (e.g., changes in liver function). Medical surveillance and other periodic preventative physical examinations are provided on a scheduled basis. Specific examination protocols as set forth by the National Institute for Occupational Safety and Health (NIOSH) may be implemented based on the individual's age, laboratory or shop processes and chemicals used, and prior medical history. Specialized examinations are provided for:

- Individuals returning to work following non-occupational illness or injury;

- Individuals returning to work following occupational illness or injury;

- Laser users;

- Individuals with significant potential for internal exposure to radioisotopes;

- Individuals using an OSHA-regulated compound that are exposed to air concentrations of that compound at or above the compound's action level (or in the absence of an action level, the PEL);

- Individuals identified as at-risk for exposure to carcinogens; and

- Individuals reporting an exposure to hazardous agents or complaining of symptoms potentially attributed to exposure of such agents.

\section{Medical Surveillance for Exposure to Hazardous Chemicals}

A medical surveillance program (medical surveillance) may include any combination of medical consultations, examinations, and clinical testing. In deciding the type of medical program, if any, that is appropriate for laboratory 
workers, it is important to keep in mind the nature of exposure conditions in a typical laboratory. Typically, chemicals used and procedures performed vary substantially between laboratories and, sometimes, within a single laboratory. Moreover, operations and chemicals may change over time. This section describes the general medical surveillance requirements under the OSHA Laboratory Standard, and LBL's policy for its laboratory and shop workers.

Consistent with DOE and UC policies, all LBL permanent personnel receive base line, pre-placement medical examinations and are strongly encouraged to participate in periodic medical examinations. However, OSHA does not require baseline medical examinations as a general policy for all laboratory personnel. Given the unpredictable array of chemicals in laboratories, general baseline examinations may not provide meaningful correlation to future adverse exposures. On the other hand, OSHA does not suggest that medical provisions are not needed under any circumstance. Clearly, an employee should receive appropriate medical attention if he/she exhibits signs of symptoms possibly related to exposure to a hazardous chemical or if he/she is subjected to events such as spills, leaks, explosions or cther unexpected occurrences where there is a likelihood of exposure to hazardous chemicals.

The OSHA Laboratory Standard provides for medical examinations in the case of symptoms and medical consultations for events (i.e., spills, leaks, fugitive emissions) or occurrences resulting in the likelihood of a significant exposure to a hazardous chemical. The medical consultation is provided for the purpose of determining the need for a medical examination.

An employee shall be afforded an opportunity to receive any examinations recommended by the physician. Medical examinations and consultations are (1) performed by or under the direct supervision of a licensed physician, (2) provided at a reasonable time and place, and (3) provided without cost to the employee.

\section{Identifying the Need for Medical Surveillance}

Generally the purpose of a Medical Surveillance Program is to watch for early health effects that may be related to an exposure and serve as an early warning of 
more serious health effects that could ensue. Occasionally, medical surveillance is utilized to help establish the dose of a chemical that the body has received.

Medical surveillance may be useful in: (1) assessing the effects of exposure to hazardous chemicals; and, (2) ruling out bodily damage/injury. Unfortunately, medial examinations are not always useful in measuring the actual dose of a hazardous chemical that the body receives. Most medical tests are not specific for the chemical exposure and tests used actually measure damage, or the early signs of disease (e.g., liver enzymes). Medical surveillance is more useful when performed in conjunction with exposure monitoring because the potential dose is easier to assess and correlate with any clinical findings that may be present.

Medical surveillance may be useful in a number of different operations and should be considered on a case-by-case basis. However, there are four occasions in which employees must be provided the opportunity to receive medical surveillance to address the potential for adverse health effects associated with exposures:

- When employees develop symptoms that appear to be associated with exposures to hazardous chemicals;.

- When employees are involved in responding to a spill, leak, explosion or other release that results in exposures to hazardous chemicals;

- When air monitoring has demonstrated exposures at or above occupational exposure limits; and

- When required by substance-specific OSHA regulations. 


\section{When OSHA Regulations Require Medical Surveillance}

The OSHA Laboratory Standard, which applies to all laboratory workers (and not strictly to shop workers) supersedes the requirements specified in substance-specific standards (e.g., for asbestos, benzene, etc.). The only requirement for laboratory personnel is to maintain exposures below occupational exposure levels. If there is reason to suspect that exposures exceed those limits, a medical ccnsultation is provided.

For non-laboratory operations (i.e., shop operations), OSHA regulations require that a medical surveillance program be implemented for two different groups of substances: regulated carcinogens and those substances that specify a surveillance program in a substance-specific OSHA standard.

Because LBL chooses to provide equal protection for all its employees all existing OSHA medical surveillance protocols will be considered, regardless of whether personnel work in laboratories or in shops. However, the likelihood of significant exposures in a laboratory setting is small.

\section{OSHA Regulated Carcinogens:}

Employees involved in handling, working with, or using any of the substances listed below in concentrations at or above the percentage amount shown must be included in a medical surveillance program. There are only two exceptions from the medical surveillance requirement:

- Operations that only handle the carcinogens in closed, sealed containers that are not opened at any point during the operations, i.e., there is no potential exposure, and

- Operations that only handle the carcinogen in concentrations below those listed below. 
OSHA Regulated Carcinogens

Name

$\begin{array}{ll}\text { 2-acetylaminofluorene } & 1.0 \\ \text { 4-aminodiphenyl } & 0.1 \\ \text { benzidine and its salts } & 0.1 \\ \text { 3,3'-dichlorobenzidine and it salts } & 1.0 \\ \text { 4-dimethylaminoazobenzene } & 1.0 \\ \text { alpha-naphthylamine } & 1.0 \\ \text { beta-naphthylamine } & 0.1 \\ \text { 4-nitrobiphenyl } & 0.1 \\ \text { N-nitrosodimethylamine } & 1.0 \\ \text { beta-propiolactone } & 1.0 \\ \text { bis-chloromethyl ether } & 0.1 \\ \text { methyl chloromethyl ether } & 0.1 \\ \text { ethyleneimine } & 1.0\end{array}$

\section{Substance-Specific OSHA Health Regulations:}

Percent concentration

0.1

0.1

1.0

1.0

0.1

1.0

1.0

0.1

0.1

1.0

The second group of substances that require medical surveillance programs are those with complete, substance-specific OSHA health regulations. Laboratories or shops that handle, work with, or use these substances in a manner which could potentially result in employee exposures at or above the action level for the substance must implement a medical surveillance program as described in the specific regulation. The action level is a trigger level which is different for each substance. Normally, employee exposure monitoring is required to measure the employees' exposure levels and to determine whether these levels exceed the action level and medical surveillance is required. The chemicals having substance-specific regulations include:

acrylonitrile

asbestos

benzene

coke oven emissions 


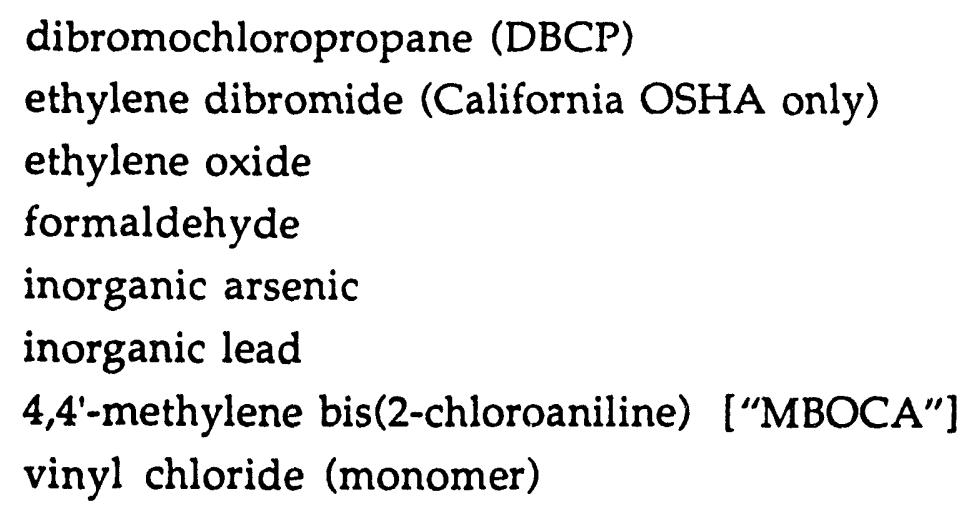

\section{Medical Consultations in Response to Symptoms and/or Chemical Releases}

Employees who develop symptoms that appear to be related to exposures associated with their jobs shall be provided the opportunity to receive medical surveillance. The content of the medical surveillance will vary depending on the nature of the exposure, the substances involved and the operation. Because of the wide diversity in types of exposures, potential adverse health effects and complicating factors, it is not possible to provide a set of simple, clear cut guidelines on when medical surveillance is needed. Each potential situation needs to be addressed on a case-by-case basis, working with LBL's Industrial Hygiene Group and Health Services.

Employees who are exposed to chemicals as a result of spills, explosions, or other releases need to be considered for medical consultation. The decision to proceed depends on a number of factors and will be decided on a case-by-case basis. For example, if a spill occurs and the proper personal protective equipment is not worn in the clean-up (contrary to the rules of this CHSP), medical consultation is warranted. Medical surveillance is normally not needed under the following circumstances:

- The release involved substances of low hazard;

- The release only caused a few seconds of exposure before the employee vacated the area; 
- Employees responding to the release used the correct personal protective equipment, were trained in the correct procedures for responding to the release, and did not experience any symptoms or adverse health effects following the response; or

- The release involved small quantities of materials which were isolated from the main work area of the laboratory or shop and only the employees with proper equipment and training were involved in responding to the incident.

Employees must inform their supervisor of adverse health effects and spills so that this information can be used, along with information on the substances being used and the operation itself, to decide if a medical surveillance is needed. Health Services notifies the Industrial Hygiene Group of potentially unsafe work conditions by sending them a copy of the Supervisor's Accident Analysis Report, which indicates their assessment that Industrial Hygiene needs to know of the accident/injury and probably needs to evaluate the work site.

\section{Exposures to Carcinogens}

The most prudent practice is to minimize all exposures to carcinogens. Although some carcinogens have established occupational exposure limits (i.e., PELs), the criteria utilized in establishing those limits may not have included carcinogen effects. And the decision to establish a new lower PEL following the discovery of carcinogenic effects is not based only on risk assessment results, but on technical feasibility to reduce exposures, cost data, etc. It takes years to change a chemical's PEL. Recent changes were made for benzene, ethylene oxide, and formaldehyde, but these are the exception.

The key to minimizing exposures to carcinogens is controlling the potential for exposures. The Carcinogen Program (Section G.2) outlines procedures to control exposures. The Industrial Hygiene Group will assess the adequacy of the control measures that each laboratory or shop utilizes by (1) conducting site inspections to observe operating procedures, and (2) reviewing written Specific Safety Procedures contained in the Facility Notebooks. 
In addition, laboratories and shops are to participate in a carcinogen exposure assessment survey. A Carcinogen Exposure Assessment Survey form (Appendix 12) is to be completed by the Principal Investigator or Laboratory/Shop Supervisor to aid the Industrial Hygiene Group in assessing the potential for ongoing and projected exposures to carcinogens. The Industrial Hygiene Group will prioritize inspections based on the survey results.

The Industrial Hygiene Group will evaluate the adequacy of control measures, and conduct air monitoring as appropriate, and refer to Health Services the names of all personnel identified as potential candidates for medical surveillance. Unfortunately, established exposure monitoring protocols and/or specific medical surveillance tests are not available for many of the carcinogens. However, employees are encouraged to participate in recommended medical surveillance programs to establish that their health status has not been affected negatively.

\section{Exposures to Reproductive Toxins}

Both men and women may be exposed to hazardous agents that pose a reproductive hazard such as infertility, hormonal changes, birth defects and genetic damage. These agents include ionizing radiation, alcohol, cigarette smoke, pharmaceuticals, and some of the thousands of different chemicals that are used in the home or workplace. Although many of these have been tested to determine whether they cause acute (immediate) effects on the body, few have been studied to see if they cause, birth defects (teratogens) or genetic defects (mutagens). Even fewer have been studied to see if they can cause infertility, reduced sperm count, menstrual disorders, or other disorders relating to reproduction.

The primary path for hazardous substances to reach an unborn child is through the placenta. Many chemicals and drugs that enter a pregnant woman's body (through breathing, swallowing, absorption through the skin, etc.) will eventually enter the mother's blood circulation, cross the placenta and thus affect the developing fetus.

In general, the important questions of exactly how much of the toxic substance that enters the mother's body will reach the fetus, or what concentration the fetus can tolerate without harmful effects are not yet answered. 
The fetus is usually most vulnerable in the early weeks of pregnancy (first 13 weeks or trimester) but may be also at risk later in pregnancy. In light of the potential harm of workplace exposures to both the pregnant woman and her developing fetus, it is very important, and is required by LBL policy, for the woman to inform Health Services of her pregnancy (or plans to become pregnant) as soon as possible, so that any necessary steps to avoid potential exposure problems can be taken. This policy exists in no way to discriminate against women; rather, it is intended to provide the pregnant woman with information about the possible hazards and her options.

Refer to Appendix 13 for a listing of reproductive toxins. 


\section{K. RECORDKEEPING}

Accurate documentation and recordkeeping of expostre monitoring, medical surveillance, and health and safety training is an important component of the LBL Chemical Hygiene and Safety Program. Further, because LBL relies on each facility to conduct hazard assessments of their operations/procedures, and to write Specific Safety Procedures (SSPs) as appropriate, the SSP documentation is paramount to the Program. The Facility Notebook is the recommended place to store those records required of the Principal Investigator or Laboratory/Shop Supervisor.

\section{Specific Recordkeeping Requirements and Responsibilities:}

- Current chemical inventories for each laboratory and shop area must be readily available for reference in the event of an emergency (pursuant to hazardous materials management requirements).

Responsibility: Principal Investigator or Laboratory/Shop Supervisor

- Documentation of Laboratory/Shop Specific Safety Procedures (SSPs) must be maintained.

Responsibility: Principal Investigator or Laboratory/Shop Supervisor

- Exposure records for hazardous chemicals and harmful physical agents (i.e., analytical reports and memos notifying employees, supervisors, and/or Health Services) will be maintained for at least 75 years (and possibly longer) in accordance with DOE orders.

Responsibility: EH\&S Industrial Hygiene Group (for all except radiation exposures)

- Names of workers working with chemicals of high acute toxicity, reproductive toxins and carcinogens must be maintained and submitted to the Industrial Hygiene Group. Records should indicate the dates the employees worked with the particularly hazardous chemicals. (Note: Do not destroy old records in the process of updating chemical use information to reflect current workers.) 
Responsibility: Principal Investigator or Laboratory/Shop Supervisor and Industrial Hygiene Group

- Medical records for employees exposed to hazardous chemicals and harmful physical agents will be maintained for the duration of employment plus 75 years in accordance with DOE orders. (Note: "Exposed to" hazardous chemicals is not necessarily the same as "working with" hazardous chemicals.)

\section{Responsibility: Health Services}

- Employee training records must be maintained and readily available. Records are to be kept for at least 75 years beyond the class or, for individuals, beyond the conclusion of their employment.

- EH\&S Sponsored Classes:

\section{Responsibility: EH\&S Training Group}

- Individual Laboratory or Shop Sponsored Classes:

Responsibility: Principal Investigator or Laboratory/Shop Supervisor

- Accident investigations must be written and retained in an organized manner.

Responsibility: Principal Investigator or Laboratory/Shop Supervisor; Occupational Safety Department, if appropriate.

- Maintenance records (i.e., inspection checks, repair work, etc.)) of safety equipment and local exhaust ventilation equipment must be maintained for at least the duration of the time that the equipment is in use.

- Eyewash and shower cl.ecks:

Responsibility: Principal Investigator or Construction \& Maintenance in conjunction with Laboratory/Shop Supervisor 
- Fire extinguishers:

Responsibility: The LBL Fire Department

- Local exhaust ventilation (e.g., lab hoods):

Responsibility: The Industrial Hygiene Group

- Biosafety cabinets:

Responsibility: The Principal Investigator or Laboratory/Shop

Supervisor. (Note: The Industrial Hygiene Group may conduct some testing but cannot "certify" these hoods.) 


\section{REFERENCES AND INFORMATION RESOURCES}

The following is a list of references and information resources that pertain to chemical hygiene and safety in the laboratory. These references can be ordered to supplement available information on chemical and physical hazards for laboratory employees. Known locations of these materials are provided for the convenience of individuals who need quick access to chemical hygiene information.

\section{Reference/Resource}

American Conference of Governmental Industrial Hygienists, Threshold Limit Values for Chemical Substances and Physical Agents in the Work Environment with Intended Changes, P.O. Box 1937, Cincinnati, OH 45201 (latest edition).

- Annual pamphlet with latest occupational exposure TLVs.

International Agency for Research on Cancer, IARC Monographs on the Evaluation of the Carcinogenic Risk of Chemicals to Man, World Health Organization Publications Center, 49 Sheridan Ave., Albany, NY 12210 (latest editions).

- Provides lists of carcinogens and criteria for their classification.

Lawrence Berkeley Laboratory, Health and Safeity Manual, Pub-3000.

- The primary LBL health and safety manual.

Lawrence Berkeley Laboratory, Guidelines for Generators of Hazardous Chemical Waste and Guidelines for Generators of Radioactive and Mixed Waste at LBL, Pub-3092.

- Training course required at LBL for generators of hazardous waste. Provides guidelines and requirements for chemical and radioactive waste collection, storage, and EH\&S pickup.

Lawrence Berkeley Laboratory, Guidelines for Waste Accumulation Areas (WAAs) at LBL, Pub-3093.

\section{Location}

Industrial Hygiene Dept., Bldg. 26
Industrial Hygiene Dept., Bldg. 26
Site-wide distribution

Site-wide distribution 


\section{Reference/Resource}

- Provides guidelines and requirements for secondary waste collection areas (i.e., where waste is transfered after removal from satellite areas and prior to transfer to the Waste Handling Facility.

Lawrence Berkeley Laboratory, Medical and Biohazardous Waste Generator's Guide, Pub-3095.

- Training course required at LBL for generators of medical and biohazardous waste. Provides guidelines and requirements for collecting, packaging, and disposing of biological waste.

29 CFR, Part 1910, Subpart Z, Government Printing Office, Washington, D.C. 20402 (latest edition).

- The Federal OSHA regulations.

Mahn, W.J., Fundamentals of Laboratory Safety, Physical Hazards in the Academic Laboratory, Van Nostrand Reinhold, NY (1991).

- General guide to the fundamentals of laboratory safety.

Mahn, W.J., Fundamentals of Laboratory Safety, Chemical Hazards Guidebook, Van Nostrand Reinhold, NY (1991).

- General guide to the fundamentals of laboratory chemical safety.

The Matheson Company, Inc. Gas Data Book, (latest edition).

- Excellent information on the safe use and handling of compressed gases, including health effects.

National Fire Protection Association, Fire Protection Guide on Hazardous Materials, (latest edition), National Fire Protection Association, Battermarch Park, Quincy, MA.

- Guide to proper fire prevention and decision-making in emergencies.

\section{Location}

Site-wide

distribution

Indusrial Hygiene

Dept., Bldg. 26

Industrial Hygiene

Dept., Bldg. 26

Indusrial Hygiene

Dept., Bldg. 26

Bldg. 26 Library;

Bldg. 90

Bldg. 26 Library;

Fire Dept., Bldg. 48 


\section{Reference/Resource}

National Toxicology Program, Annual Report on Carcinogens, Public Health Service, U.S. Dept. of Health and Human Services, Government Printing Office, Washington, D.C. 20402 (latest edition).

- Provides lists of carcinogens and criteria for their classification.

National Research Council, Prudent Practices for Handling Hazardous Chemicals in Laboratories, National Academy Press,Washington, DC, 1983.

- Recommends comprehensive procedures for the safe handling of hazardous chemicals in all types of laboratories. Addresses potential hazards from fire, explosion, and toxic substances.

NIOSH/OSHA, Pocket Guide to Chemical Hazards, U.S. Department of Health and Human Services, National Institute for Occupational Safety and Health.

DHHS (NIOSH) Publication No., 85-114, 1985.

- Quick reference for 380 chemical hazards for which there are specific federal regulations. Contains data on exposure levels, properties, incompatibilities, personal protection, and health hazards.

Ottoboni, M.A., The Dose Makes the Poison, A PlainLanguage Guide to Toxicology, Vincente Books, Berkeley, CA (1.984).

- Serves as a primer on toxicology

Public Health Service Registry of Toxic Effects of Chemical Substances, U.S. Dept. of Health and Human Services, Centers for Disease Control, National Institute for Occcupational Safety and Health, revised annually, for sale by Supt. of Documents, Government Printing Office, Washington, D.C. 20402.

- Provides very detailed toxic effects information.

\section{Location}

Industrial Hygiene Dept., Bldg. 26

Bldg. 26 Library; Donner Library; Bldg. 62 Library

Bldg. 26 Library

Industrial Hygiene Dept., Bldg. 26

Industrial Hygiene Dept., Bldg. 26 


\section{Reference/Resource}

Proctor, Nick H. \& James P. Hughes. Chemical Hazards of the Workplace, Philadelphia PA, J.B. Lippincott Co., 1978.

- Excellent source of health hazard summary information.

Steere, Norman V., CRC Handbook of Laboratory Safety, The Chemical Rubber Company, Cleveland, OH, 1971.

- A reference on first aid, biohazards, chemical and radiation hazards.

Steere, Norman V. Safety in the Chemical Laboratory (Volumes 1-3) J. Chem Ed., American Chemical Society, Easlon, PA, 1981.

- Provides general and detailed laboratory safety information.

Young, Jay A., Improving Safety in the Chemical Laboratory, John Wiley \& Sons, Inc., New York, 1987.

- Includes information on how to recognize close calls and eliminate accidents, personal protective equipment, contingency training programs, equipment and emergency checklists, and how to make sure safety objectives are met.

\section{Location}

Bldg. 26 Library;

Donner
Bldg. 62 Library;

Bldg. 50;

Bldg. 26 Library;

Donner

Bldg. 26 Library; UCB 


\section{Appendix 1}

\section{OSHA Hazard Communication Standard}

(29 CFR 1910.1200) 


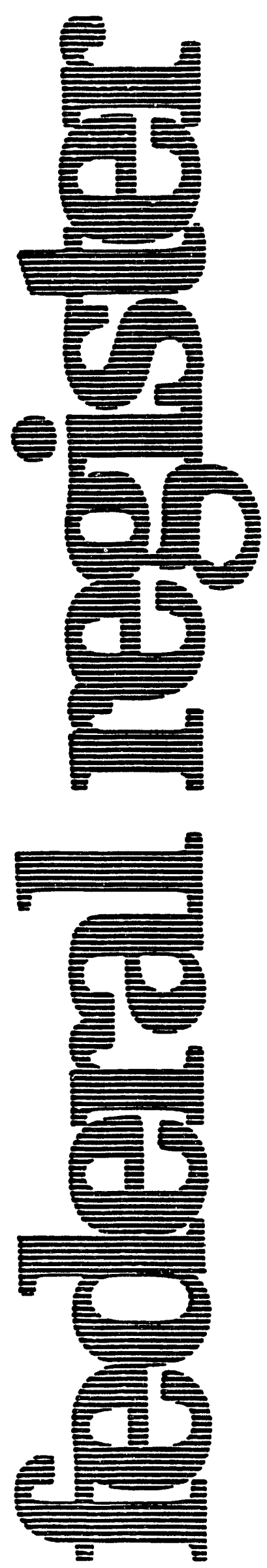

Monday

August 24, 1987

\section{Part III}

\section{Department of Labor}

Occupational Safety and Health Administration

29 CFR Parts 1910, 1915, 1917, 1918, 1926, and 1928

Hazard Communication; Final Rule 
seca 4. a Occupational Safety and Healtb Act of 1970 (20 U.S.C 053. 055. 257): Secrelary of Labor's Order No. 22-n (30 FR 8754). 4-70 (41 FR 2505\%). or Q-03 (40 FR 35736). a eppliesble.

Section 1918.90 also issued under $\$$ U.S. 553 and 28 CTR Part 1912.

PART 1926-SAFET AND HEALTH REQULATIONS FOR CONSTRUCTION

5. The authority citation for Subpart D of Part 1820 is revised to read as followe:

Aubledtr. Sec 107. Contrect Wort Hours and Salery Sundends Act (Construction Safoty Act) ( 10 U.S.C 333k recer \& a a Occupational Safety and Healtb Act of 2970 (20 US.C 653, ass. 657): Secretary of Labor'. Order No. 22-7 (30 FR 8754), Q-70 (41 FR 25050), or o-es (16 FR 35730), as applicable.

Section 192050 also kasued under B U.S.C s53 and 20 CRR Part 1911.

\section{PART 1928-OCCUPATIONAL SAFETY AND HEALTH STANDARDS FOR ARRICULTURE}

6. The authority citation for Part 1928 is revised to read as follows:

Autbority: Secs. 6 and a Occupationa Safety and Health Act of 2070 (20) U.S.C. ass. 657k Secretary of Labor's Orden 22-7) (30 FR 8731). 2-78 (42 FR 25050) or o- -3 (49 FR 25730), as applicables 20 CFR Part 1921. 533

Section 192821 also issued under 8 U.S.C

PAFTS 1910, 1915, 1917, 1918, 1924 and 1928- [AMENDED]

7. Parts 1910. 1925. 1917. 1918 and 2920 are amended by reviaing f 1910.1200 as set forth below. and by adding 18 1915.09. 191728, 1918.90. and 1920.59 to contain the identical text of the revised \& 1910.1200 . Including Apendices A. B. C and D of 1910.1200 .

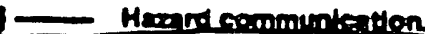

(a) Purpose. (1) The purpose of thin section is to easure that the hazards of all chemicals produced or imported are evalunted and that information concerning their hazard $b$ tranemitted to employers and employeea. This - . transerittal of information is to be, cocomplished by means of

cocoprehessive bazand communiention programs, which are to inclade . - .. .: .. contniner labeling and other forms of warning material cafety data abeets and employee training

(2) This occupational-safoty and health standard is inteaded to address comprebensively the issue of evaluating the potential bazands of chemicals, and communicating information concerning bezands and appropriate protective measures to employees. and to preempt any legal requirements of atate. or political subdivision of a state. pertaining to the subject. Evaluating the potential bezards of chemicals. and communicating information concerning hazards and appropriate protective measures to employees. may include. for example. but is not limited to. provisions for: developing and maintaining a written hazand communication program for the workplece including lists of hazardous chemicals present labeling of containers of chemicals in the workplace as well as of containers of chemicals being shipped to other workplaces: preparstion and distribution of material cafety dats sheets to employees and downstream employera: and development and implementation of employee training programs reganding bernds of chemicals and protective measures. Under section 18 of the AcL no atate or political subdivision of a state may adopt or enforce, through any court or agency. any requirement relating to the issue addressed by this Federal standard except puraunat to a Federally-approved stato plan.

(b) Scope and application. (1) This sectioa requires chemical manufacturess of importers to assess the hazands of chemicals which they produce or import. and all employers to provide Information to their employees about the bernisous chemicalo to which they are exposed. by mean of a harand communication progrnm labels and other forms of waming. material safety date sheets. and information and training In addition this section requires distributors to tranamit the required information to employers.

(2) This section applies to any chemical which is known to be present in the workplace in such a menner that employees may be exposed under normal conditions of use or in a foreseenble emergency.

(3) This section applies to laboratories only as followe

(i) Employers shall ensure that Lbel on tocoming containers of berardous chemicaln are not removed or defaced.

(ii) Employers shall maintnin any: material safety data sheets that are received with incoming ahipments of heandous chemicale and encure that they are readily accessible to laboratory employeer: and : : : :... in..

(iin Employer stall enswe that laboratory employees are apprised of the bazards of the chemicals in their workplaces in eccordance with paragraph (h) of this section.

(4) In work operation where employees onty bandle chemicals in sealed containers which are not opened under norraal conditions of use (such as are found in marine cargo bandling. warehousing, or retail seles). this section applies to these operations only as follows:

(i) Employers shall ensure that labels on incoming containers of hazardous chemicals are not removed or defaced:

(ii) Employers shall maintain copies of any material safety data sheets that are received with incoming shipments of the realed containers of bazardous chemicals, sball obtain a material safety dats sheet for sealed containers of harendous chemicals received without a material safety date sheet if an employee requests the materid safety data sbeet and shall ensure that the material safety data sheets are readily accessible during each work shift to employees when they are in their worts area(o): and

(iii) Employers shall enrure that employees are provided with information and training in accordance with paragraph (h) of this section (except for the location and availability of the written bazard communication program under paragraph (h)(1)(iii)). 10 the extent necessary to protect them in the event of a spill or leak of a bazardous chemical from a sealed container.

(5) This section does not require labeling of the following chemicals:

(i) Any pesticide as such term is defined in the Federal Inectiolde. Fungicide. and Rodenticide Act (7 U.S.C. 136 et seq.), when subject to the labeling requirements of that Act and labeling regulations bseued under that $A$ ct by the Environmental Protection Agency.

(ii) Any food. food additive, color. additive. drug. cosmetic or medical or veterinary device. including materisl intended for use as ingredients in such products (e.g. Davors and fragrances). as such terms are defined in the Federal Food Drug, and Cosmetic Act (21 U.S.C. 301 of seq.) and regulations lasued under that Act when they are subject to the labeling requiremeats under that Act by the Food and Drog Administration .

(iii) Any distilled spirite (bevernge alcobolsh inine or matt beverage. intended for nonindurtrial use, as such terms are defined in the Federal Alcohol Administretion Aet (27 US:C. 202 .et seq.) and regulationi insued inder that Act when subject to the labeling. requirement of that $A$ ct and libeling regulations trered under thit Act by the Buread of Alcobol Tobicco, and Firearmgrand.

(Iv) Any connumer product oi bezardous substance as those terms are defined in the Conoumer Product Safety Act (15 U.S.C. $205 \pi$ el seq.) and Federal Hazardous Substances Act (15 U.S.C. 1201 ef seq.) respectively, when subject to a conoumer produrt safety standard or labeling requiremeat of those Acts. of regulations issued under those Acts by 
the Consumer Product Safety

Commission.

V (6) This section does not apply to:

(i) Any hazardous waste as such term is defined by the Solid Waste Disposal

ACL as ameonded by the Resource

Conservation and Recovary Act of 1976. as ameaded ( 12 U.S.C 6901 et seq.). when subject to regulations iscued under that Act by the Enviranmental Protection Agency.

(ii) Tobacco or tobacco products:

(iii) Wood or wood producte:

(iv) Articles:

(v) Food drugs, coemetics, or alcoholic beverages in a retail establishment whicb are packaged for sale to consumers:

(vi) Foods drugs, or cosmetics intended for personal consumption by employees while in the workplace;

(vii) Any consumer product or hazardous substince as those terms are defined in the Consumer Product Safety Act (15 U.S.C. 2051 et seq.) and Federal Hezandous Substances Act (15 U.S.C. 1261 et seq.) respectively. where the employer can demonstrate it is used in the vorkplace in the same manner as normal consumer use, and which use results in a duration and frequency of exposure which is not greater then exposures experienced by consumers: and.

(viii) Any drug. as that term is defined in the Federal Food Drug. and Cosmetic st (22 U.S.C 301 et seq.), when it is in ulid. final form for direct administration to the patient fie. cablets or pills).

(c) Definitions.

"Article" means a manufactured itcm: (i) Whlch is formed to a specific shape or design during manufacture: (ii) which has end use function(s) dependent in whole or in part upon its shape or deaign during end use: and (iii) which does not release. or other:vise result in exposure to. a hasardous shemical under normal conditions of ure.

"Assiatant Secretary" means the Assistant Secretain of Labor for Occupational Safety and Health. U.S. Department of Labot, or designee.

"Chemical" means any element. chemical compound or mixture of elements and/or compounds.

"Chemical manufacturer" means an employer with a workplace where chemical(s) are produced for use or distribution.

"Chemical name" means the scientific designation of a chemical in accordance with the nomenclature system

developed by the Intemational Union of Pure and Applied Chemistry (IUPAC) or the Chemical Abstracts Service (CAS)

- les of nomenclature. or a name which

I clearly identify the chemical for the purpose of conducting a hazard evaluation.

"Combustible liquid" means any liquid hoving a nasbpoint at or above $100^{\circ} \mathrm{F}\left(37.8^{\circ} \mathrm{C}\right)$, bul below $200^{\circ} \mathrm{F}\left(93.3^{\circ}\right.$ C). except any mixture baving components with flasbpoints of $200^{\circ} \mathrm{F}$ $\left(93.3^{\circ} \mathrm{C}\right)$. or higher. the total volume of which make up 09 percent or more of the cotal volume of the mixcure.

"Commos anme" mean any designation or identification ruch as code name code mumber. trade name. brand name or generic name used to identify a chemical other then by its chemical name

"Compresued gas" meanc

(i) A ges or mixture of gases having. in a coatriner. en absolute pressure exceeding 10 pal at 70 F $\left(211^{\circ} \mathrm{C}\right)$ : or

(ii) a gas or mbture of gaces having in a contriner, un abcolute preasure exceeding 104 pai at $1300^{\circ} \mathrm{F}\left(514^{\circ} \mathrm{C}\right)$ regardless of the pressure at 70 'F (21.1 'CF or

(iii) A liquid having a vapor pressure exceeding 40 pal at $200^{\circ} \mathrm{F}\left(37^{\circ} 8^{\circ} \mathrm{C}\right)$ as determined by ASTM D-323-72.

"Container" means any bag, barrel. bottle box, can cylinder. drum. reaction vessel storage tank or the like that contains a hazandous chemical For purposes of this eection pipes or piping systems, and engines, fuel tanks, or other operating systems in a vehicle, are not considered to be containers.

"Designated representative" means any individual or organization to whom an employee gives written authorization to exercise such employee:s rights under this section. A recognized or certified collective bargaining agent shall be treated automatically as a designated representetive without regard to written employee authorization.

"Director" means the Director. National Institute for Occupational Safety and Health. U.S. Department of Hedith and Human Services. or deaignee.

"Distributor" means a businese, other than a chemical manufacturer or importer. which supplies bazardous chemicals to other distributons or to employers.

"Employee" means a worker who may be exposed to bazardous chemicals under nomal opernting conditions or in foreseeable emergencies. Worken such as office workers or bank telles who encounler hazardous chemicals only in non-routine. isolated instances are not covered.

"Emp!oyer" means a person engaged in a business where chemicals are either used distributed. or are produced for use or distribution including a contraclor or subcontractor.
"Explosive" means a chemical that cauces a sudden almost instantaneou release of pressure. gas, and heat when - ubjected to sudden sbock pressure or high temperature.

"Exposure" or "exposed" meana that an employee is subjected to a bazandous chemical in the course of employment through any route of entry (tinhilation. ingestion. skin contact or absorptron. etc) and includes potential (e.t: -coidental or possibie) exposure.

"Flammable" means a chemical that falls lato abe of the following categories:

(i) Nieroach fammable? meane an aerosol that when teoted by the method described in 16 CFR 150045. yields a Dame projection exceeting 28 inches at full vatve opening or a Darbback (a bame exteriding back to the valve) at any degree of valve opeaings

(ii) "Gas tlammable" means:

(A) A gas that at ambient temperature and presenre, forms a flammable mixture with cir at a concentration of thirteen (13) percent by volume or less: or

(B) A gas that at ambient temperature and pressure, forms a range of nammable mixtures with atr wider than iwelve (12) percent by volume. regardless of the lower limit

(iii) "Liquid Iammable" mens any liqud baving a flasboint below $100 \mathrm{~F}$ (37. 'C), except any mixture having components with flashpoints of $100^{\circ} \mathrm{F}$ (37. $8^{\circ} \mathrm{C}$ ) or higher, the total of which make up 99 percent or more of the total volume of the mixture:

(iv) "Solid. flammable" means a salid. other than a blasting egent or explosive as defined in \$ 190.209(a). that is liable to cause fire through friction absorption of moisture. spontaneous chemical change, or retained heat from manufacturing or proceseing. of which can be ignited readily and when ignited burns co vigorously and pervistently as to create a serious hazard A chemical shall be convidered to be a flammable solid if when tested by the method described in 26 CFR 1500.44. It ignites and burns with a self-sustained lame at - rate greater then one-tenth of an inch per second along its major axis.

"Flaskpoint" mean the minimum temperature at which a liquid gives off a vapor in sufficieat concentration to ignite when tested as follows:

(i) Tagliabue Closed Tester (See American National Standard Method of Test for Flasb Point by Tag Closed Tester. 211 24-1870 (ASTM D 56-79)) for liquids with a viscosity of lese than 45 Saybolt University Seconds (SUS) \&1 100 'F (37.8 $\left.{ }^{\circ} \mathrm{C}\right)$, that do not contaln suspended solids and do not heve a 
lendency to form a surfure. film under test: or

(ii) Penaky-Martens Closud Tester (See Americun Nationul Stundard Methesd of Test for Flash Point by Pensky-Martens Closed Tester. 211.71978 (ASTM D 83-70)] for liquids with : viscosity equal to or greater than 45 SUS at $100^{\circ} \mathrm{F}\left(378^{\circ} \mathrm{C}\right.$. or that contuin uspended solids. or that have teodency to form a suriace film under test: or

(iii) Setaflash Closed Tester (see American National Standard Methud of Test for Flasb Point by Setaflash Closed Tester (ASTMDD 3208-78)) .

Organic peroxides. which undergo autonccelerating thermal decompasition. are excluded from any. of the flasbpoint determination methods specified above.

"Foreseesble emergency" means any potential occurrence such as. but not limited to. equipment failure. rupture of containers. or failure of control equipment which could result in an uncontrolled release of a hazardous chemical into the workplace.

"Husardous chemical" means any chemical which is a physical hazand or a health bazand.

"Hexard warning" means any words. pictures, symbole or combination thereof appearing on a label or other. appropriate form of warning which . coavey the hesard(s) of the chemical(s) . in the container(s).

"Health bazard" means a chemiod for. which there is statintically. atgonificant. evidence based on at lenst one study conducted in eccordance with established scientific principles that acute or chronic health effects may occur in exposed employees. The term "health hazard" includes chemicals which are carcinogens loocic or highly toxic agerts. reproductive loxing. irritants. corrosivea ceasitizers. hepatotoxins, nephrotoxins. neurotoxins. agents which act on the bernatopoietic aystem, and agents which damage the lungen skin eyen or mucous membranes: Appendix A provides further definitions and explanations of the ecope of health berands covered by this section and Appendix B describes the criteris to be used to determine whether or not a chemical is to be considered hazardous for purposes of this standand.

"Identity" means any chemical or common name which is indicated on the material safety data sheet (MSDS) for the chemical The identity used shall permit cross-references to be made amons the required list of hazardous chemicals. the label and the MISDS.

"immediate use" means that the hazardous chemical will be under the vontrol of and used unly by the pernon who transiers it from a laticled container and only within the worh ahif in which it is transferred.

"Importer" means the firsi business with employees within the Cusinms Territory of the United States which receives hazardous chemicals produced in other countries for the purpose of supplying them to distributors or employers within the United States.

"Label" means any written. printed. or gruphic material. displayed on or affixed to contu'ners of hazardous chemicals.

"Material safety dath sheet (MSDS)" meuns written or printed material concernins a hazurdows chemical which is prepared in accordance with paragrnph (8) of this section.

"Mixfure" means any combination of iwo or more chemicals if the combinution is not in whole or in part. the result of e chemical reaction.

"Organic peroxide" means an organic compound that contains the bivalent - 0 O-structure and which may be considered to be structural derivative of hydrogen peroxide where one or both of the hydrogen atoms has been reploced by an organic radical.

"Oxjdizer" means a chemical other then a blasting agent or explosive as defined in $(1910.109$ (a). that indiates or promotes combustion in other materials. thereby causing fire either of itself or through the selease of oxygen or other gases.

Thyoical hasard" means a chemical for which there is seientifically valid. evidence that it is a combustible liquid. - compressed gas, explosive. nlammable. an organic peroxide. an oxidizer.

psrophoric. unstable (reactive) or waterreactive.

"Produce" mean to manufacture. procese. formulate. or repackage.

"Pyrophoric" means a chemical that will ignite spontaneoualy is ais at a temperature of $230^{\circ} \mathrm{F}\left(54^{\circ} \mathrm{C}\right)$ or below.

"Responatble party" means someone who can provide edditional informetion on the hazardous chemical and appropriale emergency procedures, if necessary.

"Specific chemical identity" means the chenical name. Chemical Abstrects Service (CAS) Registry Number, or any other information that reveals the precise chemical designation of the subetence.

"Trade secret" means any confidential formula. pattern. process. device. information or compilation of information that is used in an employer's business. and that gives the employer an opportunity to ohioin an advantse over competitors who do no know or use it. Appendix D sets out the criterin to be used in evyluating trado sr.crets.

"Unstable (reactive)" means a themical which in the pure state. or dy produced or transported. will vigorously polymerize. decompose. condense. us will become self-reactive under conditions of shocks. pressiure or temperature.

"Use" means to package. handle. reacl. or transfer.

"Water-reactive" means a chemical that reacts with water to release a gus that is either flammahle or presents a health hasurd.

"Work area" means a room or defined space in a workplace where haziardous chemicals are produced or used. and where employees are presenl.

"Workpluce" means an eatablishment. job site. or projecl. at one geographical location containing one or more work areas.

(d) Hazard determination. (1) Chemical manulacturers and importers shall evaluate chemicals produced in their workplaces or imported by them 10 determine if they are hazardous.

Employers are not required to evaluate chemicals uniess they choose not to rely on the evaluation performed by the cherieal manufacturer or importer for the chemical to satisfy thit requiremen

- (2) Chemical manufacturess. importer or eimployers evaluating chemicals shall identify and conaider the available scientific evidence concerning such hazards Fur health hazards evidence which is statistically significant and which is bised on at least one positive study conducted in ascordance with established scientific principles is considered to be sufficient to establish a hazardous effect if the results of the study meet the definitions of health hazards in this section. Appendix A shall be consulted for the scope of health hezards covered. and Appendix 8 shall be consulted for the criteria to be followed with respect to the completeness of the evaluation and the data to be reported.

(3). The chemical manufacturer. importar ne employer exaluntine chemicales ball trest the following sources as establishing thas the cherwicals listed in them are hazardaure

(i) 20 CFR Part 1910. Subpan 2 Toxic and Hazardous Substances. Occupational. Safety and Health Adminiatration (OSHA): or.

(ii) Threshold Limit Values for Chemical Substances and Physical Agents in the Work Environment. American Conference of Governmental Indu-trial Hygienists (ACCIH) (lates! editionl. 
The chemical manufacturer. importer. or employer is still responsible for evaluating the hazards associated with the chemicals in these source lists in accordance with the requirements of this standard.

(4) Chemical manufacturers. importers and employer evaluating chemicals shall treat the following sources as establishing that a cheinical is a carcinugen or potential carcinogen for hazard communication purposes:

(i) National Toxicology Program (NTP). Annual Report on Carcinogens (latest edition):

(ii) International Agency for Research on Cancer (LARC) Monogrophs (latest editions): or

(iii) 29 CFR Part 1910. Subpart 2. Toxic and Hazardous Substances. Occupational Safety and Health Administration.

Nota-The Registry of Toxic Effects of Chemicol Subsioncas published by the National Institute for Ocouparional Safery and Healit indicales whether a chemical has been found by NTP or LARC to be a potential carcinogen.

(5) The chemical manufacturer. importer or employer shall determine the hazands of mixtures of chemicals as follows:

(i) If a mixture has been tested as a whole to determine its hazands. the results of such teating shall be used to determine whether the mixture is hezardiour:

(ii) If a mixture has not been tested as a whole to determine whether the mixture is a bealth hazard. the mixture shall be assumed to present the same health bazurds as do the componeats which comprise one percent (by weight or volume) or greater of the mixture. except that the mixture sball be assumed to present a carcinogenic hazard if it contains a component in concentrations of 0.1 percent or greater which is considered to be a carcinogen under paragraph (d)(4) of this section:

(iii) if a mixture has not been tested as - whole to determine whether the mixture is a physical hazard, the cheonical manufacturer. importer. or employer may use whatever scientifically valid data io evailable to evaluate the physical bazard potental of the mixture, and.

(iv) If the chemical manufacturer. importer. or employer bas evidence to indicate that a component present in the mixture in conceatrations of lese than one perceal (or in the case of carcinogens. Jess (han 0.1 percent) could be relessed in concentrations which would exceed an established OSHA Dermissible exposure limit or ACCDH hreshold Limit Value. or could present a health bazard to employees in those concentrations. the mixture shall be assumed to present the same hazard.

(6) Chemical manufacturers. importers. or employers evaluating chemicals shall describe in writing the procedures they use to determine the harands of the chemical they evaluate. The written procedures are to be made available. upon request. to employees. their designated representatives. the Assiatant Secretary and the Director. The written description may be incorporated into the written hazard communication program required under paragraph (e) of this section.

(e) Written hazand communication progrom (1) Employere abatt develop: implement and maintain at the workplace, written haznd communication program for their workplaces which at least describes how the criteria specified in paragraphs (1). (8), and (h) of this section for labels and other forms of warning. material safety data sheets. and employee information and training will be met. and which also includes the following

(i) A list of the hezardous chemicals known to be present using an identity that is referenced on the appropriate material safety data sheet (the list may be compiled for the workplace as a whole or for individual work areas):
and.

(ii) The methods the employer will use to inform employees of the harards of non-routine taske (for example, the cleaning of reactor vessels), and the herards associated with chemicals contained in unlabeled pipes is their
work areas.

(2) Multi-mployer workploces. Employers who produce, use. or store hazardous chemicals at a workplace in such a way that the employees of other employer(a) may be exposed (for example. employees of a construction contrector working on-site) shall additionally easure that the hazand communication programs developed and implemented under this paragraph (e) include the following:

(i) The methods the empioyer will use to provide the other employer(s) with a copy of the material safety data sheet or to make it available at a central location in the workplace, for each hazardous chemical the other employer(s)' employees may' be exposed to while working:

(ii) The methode the eroployer will use to inform the other employer(s) of any precautionary measures that need to be taken to protect employees during the workplace's normal operating conditions and in foreseeable emergencies: and. (iii) The methods the employer will use to inform the other employer(s) of the labeling system used in the workplace.

(3) The employer may rely on an existing hazard communication program to comply with these requirements. provided that it meets the criteria establisbed in this paragraph (e).

(4) The employer sball make the written hazard communication program available. upon request. to employees. their designated representativea, the Ausiatant Secretary and the Director. in accordance with the requirements of 29 CFR 1810.20(e).

(I) Labels and other forms of waming. (1) The chemical manufacturer, inporter. or distributor shall ensure that each container of hazandous chemicals leaving the workplace is labeled tagsed or marked with the following

information:

(i) Identity of the hasardous chemical(s):

(ii) Appropriate hazurd warnings: and

(iii) Name and address of the chemical manufacturer. inaponer, or other responsible party.

(2) For solid mietal (such as ateel beam or metal casting) that is not exempted as an article due to its downstream use. the required label may be transmitted to the customer at the time of the intial shipment and need not be included with subsequent shipments to the same employer ualess the information on the label changes. The label may be transmitted with the initial shipment itself, or with the material eafety data sheet that is to be provided prior to or at the time of the first shipment. This exception to requiring labels on every concainer of hazardous chemicals is only for the solid metal - itself and does not apply to bazurdous chemicals used in conjunction with or known to be present with the metal and to which employees handling the metel may be exposed (for example. cutting nuids or lubricants).

(3) Chemical manufacturers. importers. or distributors shall ensure that each container of hasardous chemicals leaving the norkplace is labeled. tassed or marked in accordance with this section in a manner which does not conflict with the requirements of the Hazardous Materials Transportation ACl (49 U.S.C. 1801 et seq.) and regulations issued under that Act by the Department of Traneportation.

(4) If the hezardous chemical is regulated by OSHA in a substance. -pecific bealth standard the cbemical manufacturer. importer, distributor or employer shall ensure that the labels or 
other furms of warning used are in accordance with the requirements of that standand.

(5) Except as provided in parugraphs (D)(6) and (I)(7) the employer shall ensure that each container of hazardous chemicals in the workplace is labeled. laged or marked with the following information:

(i) Identity of the bazardoue chemical(s) contained therein: and

(ii) Appropriate basard warmings.

(6) The employer may use signs. placards. process sheets. batch tickets operating procedunes, or other such written materials in lieu of aftixing labels to individual stationary process containers as long as the allernative method identifies the containers to which it is applicable and conveys the information required by paragraph (D)(5) of this section to be on a label. The written materials shall be resdily accessible to the employees in their work area throughout each work shift

(7) The employer is not required to label portable containers into which hazardous chernicals are trensferred from labeled containers. and which are intended only for the immediate use of the employee who performs the transfer.

(B) The employer shall not remove or defece existing labels on incoming containers of hazardous chemicals. ualeas the contuines is immediately marked with the required information.

(9) The employer shall envure that labels or other forms of waming are legible in English and prominently displayed on the container. ar readily available in the work area throughout each work shift. Employers having eraployees who speak other languages may add the information in their langunge to the material presented as long as the information is presented in Englisb as well

(10) The chemical manufactures. importer. dintributor or employer need not affix new labels to comply with this section if exdsting labels already convey the required information.

\section{(B) Baterial sefety dato sheets. (1)} Chemical manufacturers and importer shall obtain or develop a material safety data abeet for eact barand ow chemical they produce or import. Emoployers ahell have a mulerial cafety dats shoet for each heourdous chemical which they

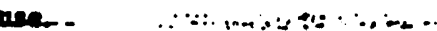

(2) Each malerial enfery data sheet thall be in English and shall coataio at least the following information

(i) The identity used on the label and excepi as provided for in paragraph (i) of this section on trede secrets:

(A) If the bazardons chemical is a single substance its chemical and common name(s):
(B) If the hazardous chemical is a mixture which bas been tested as a whole to determine its hazards. the chemical and coramon name(s) of the ingredients which contribute to these known bazands, and the common name(s) of the mixture itself: or.

(C) If the bezardous chemical is a mixture which has not been tested as a whole:

(1) The chemical and common name(s) of all ingredients which have been determined to be bealth bazards. and which comprive 18 or greuter of the composition except that chemicals identified as carcinogens under paragraph (d)(d) of this section shull be listed if the concentrations are $0.1 \%$ or greater. and

(2) The chemical and common name(a) of all ingredients which have been determined to be bealth bazards. and which comprise less than $1 \times 10.1 \%$ for carcinogens) of the mixture. if there is evidence that the ingredient(s) could be released from the mixture in concentrations which would exceed an established OSHA permissible exposure limit or ACGLH Trreshold Limit Value. or could present a health bazurd to employees: and

(J) The chernical and common name(s) of all ingredients which bave been determined to present a physical hazard when present in the onix ture:

(ii) Physical and chemical

churacteristica of the hazardous chemical (such as vapor prescure. flasb point):

(iii) The physicul hazards of the hazardous chersical including the poteatial for fire, explosion and recetivity:

(iv) The bealth herards of the barandous chemical includimg signs and symptoms of exposure, and any medical conditions which are generally recognized as being ageravated by exposure to the chemich:

(v) The primery route(s) of entry:

(vi) The OSHA permissible exposere limit ACGH Thresbold Ltmit Value. and any other exposure limlt used or recommended by the chemical manufacturer. tmporter, or employer preparing the material eafety data sheet where availabies

(vii) Whether the hazandous chemical is listed in the Netional Toxicolo. Progrem (NTP) Armual Report on Carcinogens (latest edition) or bas been found to be a potential carcinogen in the International Agency for Reseerch on Cancer (LARC) Monographs (latest editions) or by OSHA.

(viii) Any generally applicable precautions for eafe basdling and use which are known to the chemical manufacturer. importer or employer preparing the material sufely ciata shcet. including appropriate hygienic practices. protective measures during repair and maintenance of contaminsted

equipment. and procedures for clean-up of apills and leaks:

(ix) Any generally applicable cuntrol measures which are known to the chemical manufacturer. importer or employer preparing the material safely dala sheet. such as appropriate engineering controls. work practices. or personal protective equipmen:

$(x)$ Emergency and first aid procedures:

(xi) The date of prepasation of the material safety dala sheet of the last change to it: and.

(xii) The aame. address and celephore number of the chemical manufuctures. importer. employer or other responsible party preparing or distributing the material safety data sheet who can provide additional information on the hazardous chemical and appropriate emergency procedures. if necessury.

(3) If not relevant informstion is found for any given category on the moterial safety data sbeet the chemical manufacturer. imporier or employer preparing the material safety date sheet sball mark it to indicate that oo applicable information was found

(4) Where complex mixtures hove similar bazards and contents (i.e. the chemical issredients are essentially the same. but the specific composition varies from mixture to mixture). the chemical manufacturer. importer or employer may prepare one material safety datu sheel to apply to all of these similar mixtures.

(5) The chernical manufactures. importer or employer prepasing the material safety data sheet shall ensure that the informution recorded acourately rellects the scientific evidence nsed in making the berand determination. If the chemical manofacturer. troporter or employer preparing the material eafety dath sheel becomes newty aware of any significant taformation regarding the bazards of a chemical, or ways to protect againat the bezards. this new information shall be added to the material safety data sheel within three monthe. If the chemical to not currently being produced or imported the chemical manufacturer or importer shall add the information to the material safety data sheet before the cherrical is introduced into the workplace agais

(B) Chemical manufacturess or importer shall ensure that distributors and employer are provided an eppropriate material safety data sheet with their intitial shipment. and with the first shipment after a material safety 
date sheet is updated. The chemical manufacturer or importer shall either provide material safety data sheets with the shipped containers or send them to the employes prior to or at the time of the shipment. If the material safety data sheet is not provided with a shipment that hae been labeled as a hazardous chemical, the employer sball obtain one from the chemical manufacturer. importer. or distributor as soon as possible

(7) Distributors shall ensure that material safety dak sheeth and updated information are provided to other distributors and employern Retail distributors which sell hazardous chemicals to commercial customers sball provide a material safety data sheet to such employer upon request. and shall post a sign or otherwise inforw them that a material safety data sheet is available. Chemical manufacturers. importers. and distributor need not provide material asfety data sheets to retail distributors which have informed them that the retail distributor does not sell the product to commercial customer or open the sealed container to use it in their own workplaces.

(B) The employer shall maintein copies of the reguired material safety data sheets for each hazardous chemical in the workplace. and shall ensure that they are readily accessible during each work shift to employees when they are in their work area(s).

(8) Where employees must travel between workplaces during a workshif. ie. their work is carried out at more than one geographical location, the material safety data sheets may be kept at a central location at the primary workplace facility. In this situation. the employer shall ensure that employees can immediately obtain the required information in in emergency.

(10) Material safety data sheet may be kept in any form. including operating procedurea, and may be desigmed to cover groups of hazardous chemicals in a work area where it may be more appropriate to address the hazards of a process rather than individual hazardous chemicals. However, the employer shall ensure that in all cases the required information is provided for each bazardous chemical and is readily -ccessible during each work shift to employees when they are in in their work areas (s).

(11) Material safety data sheets shall also be made readily available. upon request to designated representatives and to the Assistant Secretary, in accordance with the requirements of 29 CFR 1910.20 (e). The Director shall also re given access to material safety data sheets in the same manner. (h) Employee informacionand uaining. Employen shall provide employees with information and training on hazardous chemicals in their work area at the time of their initial assignment. and whenever a new hazard is introduced into their work area.

(1) Information. Employees shall be informed of:

(i) The requirements of this section:

(ii) Any operations in their work area where hazardous chemicals are present and.

(iii) The location and availability of the written bazand communication program including the required list(a) of hazardous chemicala and material safety dats sheets required by this section.

(2) Troining. Employee training shall include at least

(i) Methods and observations that may be used to detect the presence or release of a hazardous chemical in the work area (such as monitoring conducted by the employer. continuous monitoring devices, visual appearance or odor of hazardous chemicais when being released etc.

(ii) The physical and bealth hazards of the chemicals in the work area:

(iii) The meaisures employees can take 10 protect themselves from these hasards, inchuding opecific procedures the employer has implemented to protect ermployees from exposure to hazandous chemicale, such as appropriate work practices, emergency procedures, and personal protective equipment to be used: and.

(iv) The details of the hazand communication program developed by the employer, including en explanation of the labeling system and the material safety datn sheet and bow employees can obtain and use the appropriate hazard information.

(i) Trode secrets. (1) The chemical manuracturet, importer, or employer may withhold the specific chemical Identity, Inciuding the chemical name and other specific identification of a hazardous chemical from the material safety data sheet. provided that:

(i) The claim that the informetion withibeld is a trade secret can be supported:

(ii) Information conlained in the material safety data sheet concerning the properties and effects of the hazardous chemical is disclosed:

(iii) The material safety data sheet indicates that the specific chemical Identity is being withheld as a trade secreti and.

(iv) The specific chemical identity is made available to health professionals. employees. and designated representatives in accordance with the applicable provisions of this paragraph.

(2) Where a treating phyrician or nurse determines that o medical emergency exists and the specific chemical identity of a bazardous chemical is necessary for emergency or first-aid treatment, the chemical manufacturer, importer, or employer shall immediately disclose the specific chemical identity of a trade secret chemical to that treating physician or nurse, regardless of the existence of a written statement of need of a confidentiality agreement. The chemical manufacturer, importer, or employer may require a written statement of need and confidentiality agreement is accordance with the provitions of paragraphs (i)(3) and (4) of this section. as soon as circumstances permil.

(3) In non-emergency situations, a chemical manufacturer, importer, or employer shall, upon requesh dieclose a specific chemical identity, otherwise permitted to be withbeld under paragraph (i)(1) of this section. to health professional (i.e. physician. industrial bygienist. toxicologist. epidemiologist or occupational bealth nurse) providing medical or other occupational bealth services to exposed employee(s), and to employees or deaignated representatives. if

(i) The request is in writing.

(ii) The request describes with reasonable detatl one or more of the following occupational bealth needs for the triformation:

(A) To assess the hazards of the chemicals to which employees will be exposed:

(B) To conduct or assess sampling of the workplace atmosphere to determine employee exposure levels:

(C) To conduct pre-assignment or periodic medical surveillance of exposed employees:

(D) To provide medical trestment to exposed employees:

(E) To select or assess appropriate personal protective equipment for exposed employees:

(F) To deaign or assess engineering controls or other protective measures for exposed employeex; and.

(G) To conduct studies 10 determine the bealth effects of exposure.

(iii) The request explains in detail why the disclosure of the specific chemical identity is essential and that in lieu thereof. the disclosure of the following toformation to the bealth professional, employee, or designated representative. would not satisfy the purposes described in paragraph (i)(3)(ii) of this section: 
The determinution of occupational health hurands is complicated by the fact thut many f the effects or aigns and aymptoms occur commonly to non-occupationally exposed populations 20 that effects of exposure are dificult to veparete from oormally occurrisg Illoesser. Occasionally. a substance causes on effect that is rarely seen in the population at large, such as angiourcomas cauned by vinyt chloride exposure. thus matoing it ensier to ascertain that the occupational exposure wus the primary causative factor. More ofien. however, the effects are common. such as luns cancer. The situation is further complicated by the lact that most chemical have sot been adequately teated to determine their health hazard poteatial and data do not exist to substantiate these effects.

There bave bees many attempts 10 calcgorize effects and to define them to various ways. Generally, the terms "acute" and "chronic" are used to delineate between effects on the basis of severity or durntion "Acule" effects usually occor rapidly as a resalt of shurt-kerm exposures. and are of short durutioa. "Chronic" effects generally oceur as a result of long-term exposure. and are of long duration.

The scute effects referred to mant irequently are thuse defined by the Apericas

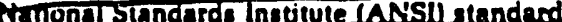

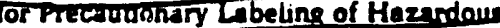

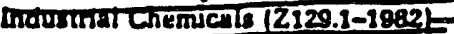

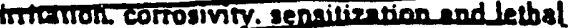
dnse. Alhough these are importnat bealth Itretr. they do aot adequately cover the vasiderable range of ecute efiects which ay occur es a reault of occupational exposure, such as. for example narcosis.

Similarly. the lerm chronic effect is oftura used to cover only eareinogeniatty. teratogenicity, and onutugenicity. These effects are obviounly 1 concers in the workplace. but again do sos edequately cover the area of chronic effects. excluding. for example. blood dyscrasine (such as enemiat chronic bronchitis and liver atrophy.

The soul of defining preciecly, in meaturable terms. every possible bealth effect that may occur is the workplace us a result of ehemical exposures cannot realistically be accomplished. This does not negate the aeed for employees to be thiformed of auch effecte and protected from them Appeodix B which is aleo mandatory. outlines the principles and procendures of herardous asecrement.

For purposes of this eection any cherricals which meet any of the lollowing definitione. as determined by the eriteria cel forth tio " Appendix B are bealth besarda:

$\rightarrow$ 1. Carcinogen: A chemical is considered to be a carcinogen if:

(a) Il bes been ovaluated by the International Arewcy for Research on Cancer (URC) and found to be a carcionogen ac potential carcinogers or

(b) If lo listed as a carcinogen or potential carcinogen in the Annual Report on Corcinogens published by the National Toxicology Program (NTP) (Inteat editions or.

(c) It is regulated by OSHA as a uncinogen. $\rightarrow 2$ Corrosive: A chemical that cuuses visible Jestruction of, or irrevernible alterations in living tissue by chersical actios at the aite of contact For example. chemical is conaridered to be corrosive if when teated on the tintact akin of albino rabbits by the method dencribed by the U.S. Department of Transportation to Appeadix A to 40 CrR Part 173. It destroys or chinges irreversibly the structure of the tissoe at the site of contact following an exposure period of four bourn. This term shall not refer to action on innimate murfaces.

73. Highly foxic A chemical falling within any of the following categories:

(a) A chemical that has a median lethal doee (LDo) of 50 milligrams or leas per kilogram of body weight when administered orally to albino rnts weighing between 200 and 300 grams each

(b) A chemical that bes a medien lethal doee (LDn) of 200 milligrams or leas per kilogram of body weight when administered by continuous contact for 24 hours (or less if death occurs within 24 hours) with the bare abio of albino rabbite weighing between two and three kilograms each.

(c) A chemical that bas a medien lethal concentretiun (LCN) in ats of 200 parts per million by volume or less of gus or vapor. of 2 milligrams per liter or less of anish fume. or dust. when adminiatered by continuous inhulation for one hour (or less if deatb occurs within one hour) to albino rets weighing between 200 and 300 greme each.

-1. Istilont: A chemical which is not corrosive. but which causes a revernible inflammatory effect on tiving tiesue by chemical ection at the site of contact A chemical is a okin tritant if. whea teated on the titact akin of albiao rabblt by the methods of 16 CFR 1500.41 for fow hour exposure or by other appropriate lectniques. it result is an empirical score of five or more. A chemical is an eye irritent if 10 determined under the procedure tioted in 10 CFR 1500.42 or other appropriate techniques. $\rightarrow$ S. Sensitizer. A chemical that causes a subateatial proportion of exposed people or animalo to develop an allergic renction in normal thave after repeated exposure to the chemical.

$\rightarrow$ a. Toxic A chomical falling within any of the following categories:

(a) A chemical that has a modins lethal does (LDD) of wore than 80 millifreme pes kilogram but not more than 500 milligram per idlogram of body weisht wben administered orally to albino rate weighing between 200 and 300 grome each

(b) A chemical that bas a medinn lethal does (LDn) of more then 200 milligrame pes tyllogram but not more then 1000 milligrame per bilogrem of body weight when administered by continuous cortact for 24 hour (or lees if death occars withis 24 bours) with the bare akin of albino rabblts weighiog between swo and three kilograme erch.

(c) A chemical that has a median lethal concentration (LC) in air of more then 200 parte per million but sol more than 2000 parts per million by volume of gas or vapor. or more than two milligrame per liler but no more than 20 milligrams per liter of miat. fume, or dust when administered by contlauous inbalation for one bour for leas if death occurs within one hour) to albloo rats weighing between 200 and 300 grams each

$\rightarrow$ 7. Target orgen effocte The following is a target organ categorization of effects which may occor. including examples of sigme and aymptome and chemicale which bave been found to canse such effects. These examples an presented to llistrate the rage and diversity of effects and bararda found to the workplace. and the broud ecope employere must consider to this area. bot are not Intended to be all-inctuaive.

- Hepalotexins: Chemicals which produce liver damage

Sigas \& Symptoms: Jeundices liver ealargement

Chemicals: Carbon tetractilorides nitrosamines

b. Nephroloxdax: Chemicalo which produce kidney demage

Sigma A Symptome: Edema: protelauric

Chemicale: Helogenatud hydrocarbones nonimm

c. Neurotoxina: Chemicale which produca their primary toxic effects on the nervous oystem

Sigon \& Symptome: Narcosic behaviond changer decreses in motor function Chemicals: Mercury; carbon disulfide

d. Agents which act on the blood or hemstopoietic system: Decrease bemoglobin function: deprive the body tinsues of oxyzea

Stone symptome: Cyanodis: lose of consciouneses

Chemieals: Carbos mosoxider cyaniden

e. Apents which damage the lung Chemicalo which britate or demage the puimonary tianue

Slowe \& Symptoms: Cough: thgtneses to chest: shartness of breach Chemiealn: Sullica: asbestos

1. Reproductive toxins: Chemicalo which affect the reproductive capabilidies locluding chromosomal damage (mutattons) and effects on fetraes (lerntogenesis)

Signe \&ymptoms: Birth defecta: eterility Chemicale: Lead; DBCP.

8. Cutaneous besarde: Chemicals which affect the dermal layer of the body Signe it Symptome: Defatting of the eltere menex trithation

Chemienle: Ketoners chlorinated compounda

h. Eya herands: Chemicals which affect the eye or visual capacity

Sime \& Symptoms: Confenctivitic cormeal damage Chemicalv: Ongenic solventerencide

Appeodix B 60 \&__ Harrand

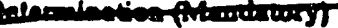

The quality of a hezand commmication program is largely dependert upon the adequacy end accurecy of the hezend delermination. The berand determinntion requirement of this stuadard is performance oriented Chemical manufacturese, importers. and employers evalueting chemicals are not required to follow any specific methods for 
determining harands but they must be able to demonstrate that they have adequately ascertained the bazards of the chemicals produced or imported in eccordance with the criterie set forth in thls Appendix.

Haserd evaluation is a proceas which relies beavly on the profezsional judgment of the evaluntor. particularly in the ares of chronic harenda. The performance-orientation of the barand determination does not diminisb the duty of the chemical manufacturer. importer or employer to conduct a thoroush evaluation examining all relevent dath and producing a scieatifically dofensible evaluation for purposes of this standard the following critoris ahall be ueod in matring busard determinations that moet the requirements of this stundard

1. Corcinogenicity: As described in paragraph (d)(1) and Appendix A of this cection a determination by the National Toxicology Program, the intermational Arency for Research on Cencer. or OSHA that a chemical is a carcinoged or potential carcinogea will be considered conclusive evidence for purposes of this section.

2 Human doto. Where available epidemiological atudies and case repors of adverse bealth effects shall be conaidered in the evaluntion.

3. Animal doto: Human evidence of healtb effects in exposed populations is generally not available for the majority of chemicals produced or aied in the workplace. Therefore. the evailable results of tordcological centing in animal population chall be wed to predict the bealth effects that moy be experienced by exposed worters. In particular. the definitions of certate acute basurds refor to specific animal ceating reoults (ses Appendix A).

4. Adequecy and reporting of data. The results of any studies which are designed and conducted according to ealublished scientific principles. and which report statistically significant conclusions regarding the health effects of a chernical shall be a sufticient bauis for a hasard determination and reported on any material safoty date theet The chemical maxufacturer, importer. of employer may also report the results of othes ocientifically valid studies which tend to refute the findings of bezand.

l Appodix C lo / - Information sourar

Tre tollowing is a list of avillable date cources which the chemical manufacturet. importer. distributor, or employer may wist to consult to evaluate the barnds of . chemicals they produce or import: Any toformation in thedr ows company

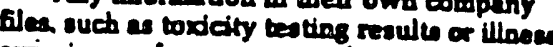
experienca of company employere.

- Any toformation obentoed from in cupplier of the chemical euch es mnleriel cafoty dat sheets or product eafety bulletim.

Any pertineal information obtained from the following source list (latest editions should be neod:

Condeneed Chemical Dictionary

Van Nostrand Reinhold Co. 235 Weat soth Street New York. NY 100020.

The Merch Index: An Encyclopedio of Chemicols and Drem.

Merck and Company. Inc 128 E. Lincolo Aire. Rabway. NJ 07005 .
IARC Monographs on the Evaluation of the Carcinogenic Risk of Chemicals 10 Man Geneve: World Health Organization. International Asency for Research on Cencar. 1972-Preaent (Muldvolume wort). Summaries are available in oupplemeat volumea. 49 Sheridan Street. Abaray. NY 22210 .

Industrial Hyziene and Toxicology, by FA Patty

Johe Wiley a Sone Ine New York. NY (Muldvolume work).

Clinical Taxicology of Commercial Products

Gleason. Gosselin and Hodge

Casarett and Doull's Taxicology, The Basic Science of Poisons

Doull. Kanseen and Aindur. Macmillan Publishing Co. lne New York NY.

Industrial Taxicology, by Alica Hemilion and Harriet L Handy

Publishing Sciences Group. Inc Acton. MA.

Taxicology of the Eye. by W. Morton Grant Charles C. Thomae, 301-329 East Lawrence Avenue. Springtield IL.

Rocognition of Healeh Harands in Industry William A. Burgesen Joho Wiley and Sons. 605 Thind Avenue. New York. NY 100358

Chemical Hezands of the Workplace

Nick H. Proclor and James P. Hughes. I.P. Upincott Company, 0 Winchester Terrace. New York MY 10022

Handbook of Chemistry and Physic

Chemical Rubber Company, 10002 Cranwood Parkway, Clevelend. OH 4122.

Threshold Limit Values for Chemieal Subrtonces and Physical Agents in the Wort Environment and Biological Exposure Indices with Lntended Changes American Conference of Covernmental Induatrial Hypieniste (ACCaH). 0500 Glenway Avenuc, Bldg. D-8. Cincinnat. OH 45211.

Information on the phyaical bezards of chemicals may be found in publication of the National Fire Prolection Association. Boston MA

Nota-The following documeate may be purchaeed from the Superintendeat of Documeate. U.S. Covernment Printing Office. Washington. DC 20102

Occupotional Health Cuidalines NIOSH/OSHA (NIOSH Pub. No. 21-223) NIOSH Pockel Guide 10 Chemicol Haso NOSH Pub. No. as-114

Rezintry of Toxic Effects of Chemical Subslances KTECD

MOSH Pub. Na So-ion

Miscellanowe Documents publiahed by the National Inatitute for Occupational Safoty and Hedtr.

Coliarla documente

Epedal Harand Reviown.

Ociapational Harard Anecarmerata Current Intelligence Bullotion.

OsHA's Ceneral induitry Standards (20 CFR. Part 1010)

NTP Annual Report on Corcinogens and Summary of the Annual Report on Corcinogene.

Nattonal Technical Loformation Service (NTIS). 8285 Port Royal Road Epringfield VA 22102: (700) $107-4050$
Blescorapamc Data Buses

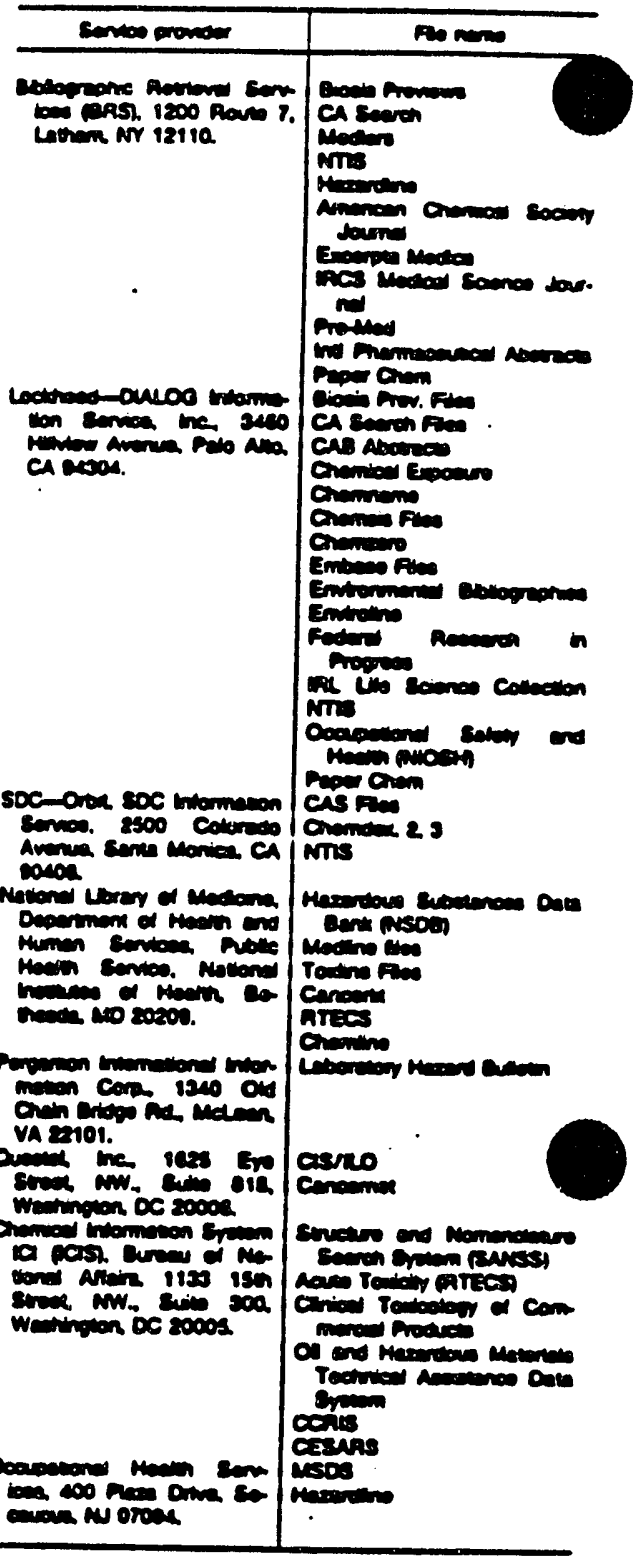

Appeadix D lo \& Secot" Mandation) Dofinition of Trade

Tho tollowing is a reprint of the

Revtotement of Torts sectlod 757, commeat b (1050):

b. Definition of trode secrie $A$ trade eccret may conaiat of any formula pattern device or compilation of information which is nead in ons's busineach and which gives bim on oppertenity lo oblesio an adventrige over compettars who de nol brow or wa ll. II may be a formula for a chemical coupound. proceses of minnufacturing, treating or preserving materials a pattera for í mectinge or other devica, or a lis of customers. It differs from other secret information the bublnese (see 1750 of the Restolemont of Torte which is not included in this Appendix) In that it is not simply information as to ingle or ephemeral events in the conduct of the businese ae, for example, the amount or 
other terma of a eccest bid for a contract or the ealary of cerris employeee, or the security imvestments made or contemptated. of the date fixed for the announcement of a new policy or for bringine out a new model or the like. A trade secret is a procese or device for continuous use in the operations of the businese Generalty if relates to the production of gooder en for example. machine or formula lor the production of an article It any. howmet, selate to the cale of soods or to other opentions in the businese. such as a code for determining discounte. rebules or other concemions in a price lint or

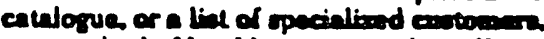
or a method of beotkecping or otber offices mangemert

Secrecy. The mubinat matter of a trade cecret must be ceare Metters of public bnowledge or of gexerd tmowledge in an inchintry canool be appropriated by one ae bis eecrel Matters which are completely diadoesed by the goods which one mertents cansor be bis secel Substantially. \& trade cecret is known only in the particular busisens is which if is ueed If is not requinite that only the propinitor of the busibeas bow il He may, withoul losing bis protection. cominunicate th to employes invotved to ite use. He may tikewiee commonicate th to others pledged to eecrecy. Others may abo know of it indepeadently. ar for example. when they have diecovered the procese or formula by indepeodent invention and are keeping it secret. Nevertheless. a substantial element of secrecy must exist. so that except by the use of improper meane there wowld be dificelty in acquiring the information. An exact definition of a trade secret is not ponable. Some fector to be considered in detesmining whether given toformation is one's trade secrel are: (1) The extent to which the finformation is known outaide of hip busineser (2) the extemt to which it tis boow by employees and others invotved in tise butiness: (3) the extent of measures takes by him to gund the secrecy of the information: (4) the value of the information to bim and his competitors: (S) the amount of effort or money expended by him to developing the information: (0) the ease or difficulty with which the information could be property acquired in couplicated by others.
Novelty and prior are A trade wecret may be a device or proceses which to paleatabte: but If need mot be thal It may be a device or proceses which is denty anticipalod to the prior ext or ooe which to meredy a mechnorical improvement that a good mechanic cen make. Novelty and imention are not requivite for a trede secret as they are for patentability. These requirements are casentid in patentabillty because a patent protect. egainet unliceneed ves of the patented device or process even by oas who diecovers it property through indepeadeat revarch The petent manopoly is e rewand to the inventoc. But roct is not the cree with a trude cecose. Its protection is not based case policy of rewerding or acterwiea eocormeging the development of eecret prociences of devices The protection is mereh againce breach of toth and reprebentible menos of learoing another's cecret for this timited protection is is sot appropriate to require also the lind of novelfy and invention which is a requinite of pateatibillty. The nature of the eceret is. howrver. an importent fector to detersining the kind of relief thet is appropriate egainat ooe who is eabject to bability under the rule staled to this eection. Thuse if the cocrer cansioces of a device of process which is a novel tovention coe who acquires the secret wrengfully is ordianily enjoined from further wee of if and is required to accouns for the profits derived from bis paat use 41 on the other hand the secret comint of wecharieal improvement that a good mechanic cas make without resort to the socrel the wrongdoer's hability may be limited to darreger and on thjunctios egainst furture use of the improvemeats mede with the aid of the secret may be inspropriate.

2. Section 1915.97 would be revised to read as followr:

\section{L15er Hadth and eentertion}

The provisions of this section shall apply to ship repeiring ahipbuilding and shipbreaking except where indicated otherwise.

(a) The employer shall provide all necessary controls, and the employees shall be protected by suitable personal protective equipment against the hazands identified under 1915.99 of this part and those hezands for which specific precsutions are required in Subparts B, C and D of this part.

(b) The employer shall provide adequate wathing fecilities for employees engaged in the application of paints or contings or in other aperations where contaminares can by ingestion or abeorption be detrimernal to the beath of the employerea. The employer ahall encourage good persoad bygieas. . prectices by toforming the employees of the beed for reworing surface contaminants by thorough washing or bands and face prior to enting or smaking.

(c) The employer sball not permit employees to eat or amoke in areas undergoing surface preparation or preservation or where shipbrealbing operations produce atmorpheric contaminante.

(d) The employer sball not permit eroptoyee ergeged in ship repair work on a vessel to work in the immediate vicinity of uncovered garbage and shall eneure that employees working beneath or on the outboand side of a vessel are not subject to contamination by drainage or waste from overboand discharges.

(c) No minor noder 18 years of age chall be employed in shipbreaking or related employments.

9. Section 192822 would be ameaded by adding paragraph (a)(5) as followe

1802821 Applicable vtandarde in 29 CFR Pant 1010.

(в) - -

(5) Hazard communication1910.1200 .

[FR Doc. 87-19137 Filed 0-19-80; Q.45 am]

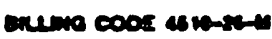


Appendix 2

OSHA Laboratory Standard

(29 CFR 1910.1450) 

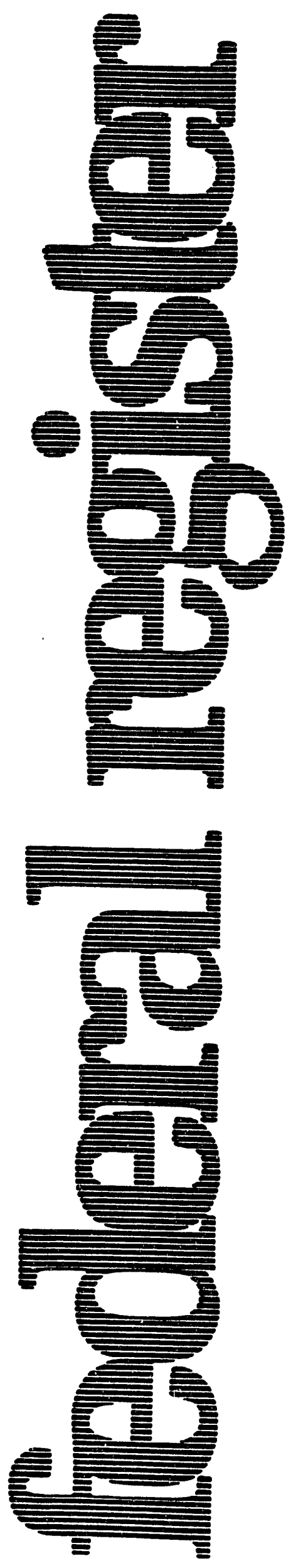

Wednesday

January 31, 1990

\section{Part II}

\section{Department of Labor}

Occupational Safety and Health Administration

\section{CFR Part 1910}

Occupational Exposures to Hazardous

Chemicals in Laboratories; Final Rule 
ensuring that their standards are at least as effective as the Federal standard.

The 25 States with their own OSHA. approved occupational safety and health plans musi adopt a comparable standard within six months of publication of a final rule. The States are: Alaska. Arizona. California. Connecticut. Hawaii. Indiana. Lowa. Kentucky. Maryland. Michigan. Minnesota. Nevada. New Mexico. New York. North Carolina. Oregon. Puerto Rico. South Carolina. Tennessee. Utah. Vermont. Virginia. Virgin Islands. Washington. Wyoming. For New York and Connecticut. plans cover only state and local government employees. Until such time as a State standard is promulgated. Federal OSHA will provide interim enforcement assistance. as appropriate. in these States.

\section{Autbority and Sigaature}

This document was prepared under the direction of Gerard F. Scannell. Assistant Secretary of Labor for Occupational Safety and Health. U.S. Department of Labor. 200 Constitution Avenue NW.. Washington. DC 20210. Pursuant to sections $6(b)$ and $8(c)$ and $8(8)(2)$ of the Act. OSHA hereby amends 29 CFR part 1910 by adding a new 1910.1450 as set forth below.

\section{List of Subjects in 29 CFR Part 1910}

Laboratories. Occupational safety and health.

Signed a Washington. DC. this 22nd day of january 1990.

Garard F. Scanoell.

Assistant Secretory ior Occupational Safety and Health.

Part 1910 of title 29 of the Code of Federal Regulation (CFR) is hereby amended as follows:

\section{PART 1910-OCCUPATIONAL SAFETY AND HEALTH STANDARDS}

1. The authority citation for part 1910. subpart $Z$ is amended by adding the following citation at the end. (Citation which precedes asterisk indicates general rulemaking authority.)

Authority: Secs. 6 and a. Occupational Safety and Health ACL 29 U.S.C. 655. 657; Secretary of Labot's Orders Nos. 12-71 (38 FR 8751). 8-76 (41 FR 25059). or 9-63 (48 FR 35736). as applicable: and 29 CFR part 1911. - Section 1910.1450 is also issued under sec. $6(b) .8(c)$ and $8(8)(2)$. Pub. L 91-506. B4 Stat. 1593. 1599. 1600: 29 U.S.C. 655. 657

2. Section 1910.1450 is added to subpart Z. pare 1910 to read as follows:

\$ 191.1450 Occupational exposure to hezardous chemicals in leboratories.

(a) Scope and opplication. (1) This section shall apply to all employers engaged in the laboratory use of hazardous chemicals as defined below.

(2) Where this section applies. it shall supersede. for labora tories. the requirements of all other OSHA health standards in 29 CFR part 1910. subpart Z. except as follows:

(i) For any OSHA health standard. only the requirement to limit employee exposure to the specific permissible exposure limit shall apply for laboratories. unless that particular standard states otherwise or unless the conditions of paragraph (a)(2),iii) of this section apply.

(ii) Prohibition of eye and skin contact where specified by any OSHA health standard shall be observed.

(iii) Where the action level (or in the absence of an action level. the permissible exposure limit) is routinely exceeded for an OSHA regulated substance with exposure monitoring and medical surveillance requirements. paragraphs (d) and (g)(I)(ii) of this section shall apply.

(3) This section shall not apply to:

(i) Uses of hazardous chemicals which do not meet the definition of laboratory use. and in such cases. the employer shall comply with the relevant standard in 29 CFR part 1910. subpart 2 even if such use occurs in a laboratory.

(ii) Laboratory uses of hazardous chemicals which provide no potential for employee exposure. Examples of such conditions might include:

(A) Procedures using chemically. impregnated test media such as Dip. and-Read tests where a reagent strip is dipped into the specimen 10 be tested and the results are interpreted by comparing the color reaction to a color chart supplied by the manufacturer of the test strip: and

(B) Commercially prepared kits such as those used in performing pregmancy tests in which all of the reagents needed to conduct the test are contained in the kil.

\section{(b) Definilions-}

"Action level" means a concentration designated in 29 CFR part 1910 for a specific substance. calculated as an eight (8)-hour time-weighted average. which initiates certain required activities such as exposure monitoring and medical surveillance.

"Assistont Secretory" means the

Assiatant Secretary of Labor for

Occupational Safety and Health. U.S.

Department of Labor. or designee.

"Carcinogen" (see "select carcinogen").

"Chemical Hugiene Officer" means an employee who is designated by the employer, and who is qualified by training or expenence. 10 provide lectinical guidance in the development and implementation of the provisions of the Chemical Hygiene Plan. This definition is not intended to place limitations on the position descnption or job classification that the designated indvidual shall hold within the employer's organizational structure.

"Chemical Hygiene Plan" means a written program developed and implemented by the employer which sets forth procedures. equipment. personal protective equipment and work practices that (i) are capable of protecting employees from the health hazards presented by hazardous chemicals used in that particular workplace and (ii) meets the requirements of paragraph (e) of this section.

"Combustible liquid" means any liquid having a flashpoint at or above $100^{\circ} \mathrm{F}\left(37.8^{\circ} \mathrm{C}\right)$. but below $200^{\circ} \mathrm{F}$ (93.3 $\left.{ }^{\circ} \mathrm{C}\right)$. except any mixture having components with flashpoints of $200^{\circ} \mathrm{F}$ $\left(93.3^{\circ} \mathrm{C}\right)$, or higher. the total volume of which make up 99 percent or more of the total volume of the mixture.

"Compressed gos" means:

(i) A gas or mixture of gases having, in a container. an absolute pressure exceeding 40 psi at $70^{\circ} \mathrm{F}\left(21^{\circ} \mathrm{C}\right)$ : or

(ii) A gas or mixture of gases having. in a container. an absolute pressure exceeding 104 psi at $130^{\circ} \mathrm{F}\left(54.4^{\circ} \mathrm{C}\right)$ regardless of the pressure at $70^{\circ} \mathrm{F}$ (21.1 'C); or

(iii) A liquid having a vapor pressure exceeding $40 \mathrm{psi}$ at $100^{\circ} \mathrm{F}\left(37.8^{\circ} \mathrm{C}\right)$ as determined by ASTM D-323-72.

"Designated area" means an area which may be used for work with "select carcinogens." reproductive toxins or substances which have a high degree of acute toxicity. A designated area may be the entire laboratory, an area of a laboratory or a device such as a laboratory hood.

"Emergency" means any occurrence such as. but not limited to. equipment failure. rupture of containers or failure of control equipment which results in an uncontrolled release of a hazardous chemical into the workplace.

"Employee" means an individual employed in a laboratory workplace who may be exposed to hazardous chemicals in the course of his or her assignments.

"Explosive" means a chemical that causes a sudden. almost instantaneous release of pressure. gas. and heat when subjected to sudden shock. pressure. or high temperature.

"Flammable" means a chemical that falls into one of the following categories: (i) "Aerosol. flommable" means an aerosol that. when tested by the method described in 16 CFR 1500.45 , yields a 
flame protection exceeding 18 inches at full valve opening, or a nashback (a Name extending back to the valve) at any degree of valve opening:

(ii) "Gas, flammable" means:

(A) A gas that, at ambient temperature and pressure, forms a nammable mixture with air at a concentration of 13 percent by volume or less: or

(B) A gas that, at ambient temperature and pressure, forms a range of flammable mixtures with air wider than 12 percent by volume, regardless of the lower limit.

(iii) "Liquid, flammable" means any liquid having a flashpoint below $100^{\circ} \mathrm{F}$ $\left(37.8^{\circ} \mathrm{C}\right)$, except any mixture having components with flashpoints of $100^{\circ} \mathrm{F}$ $\left(37.8^{\circ} \mathrm{C}\right)$ or higher, the total of which make up 99 percent or more of the total volume of the mixture.

(iv) "Solid, flammable" means a solid. other than a blasting agent or explosive as defined in $\$ 1910.109(a)$, that is liable to cause fire through friction, absorption of moisture, spontaneous chemical change. or retained heat from manufacturing or processing, or which can be ignited readily and when ignited burns 80 vigorously and persistently as to create a serious hazard. A chemical shall be considered to be a flammable solid if, when tested by the method described in 16 CFR 1500.44, it ignites and burns with a self-sustained flame at a rate greater than one-tenth of an inch per second along its major axis.

"Flashpoint" means the minimum temperature at which a liquid gives off a vapor in sufficient concentration to ignite when tested as follows:

(i) Tagliabue Closed Tester (See American National Standard Method of Test for Flash Point by Tag Closed Tester, Z11.24-1979 (ASTM D 56-79))-for liquids with a viscosity of less than 45 Saybolt Universal Seconds (SUS) at $100^{\circ} \mathrm{F}\left(37.8^{\circ} \mathrm{C}\right)$, that do not contain suspended solids and do not have a tendency to form a surface film under test: or

(ii) Pensky-Martens Closed Tester (see American National Standard Method of Test for Flash Point by Pensky-Martens Closed Tester, Z11.7-1979 (ASTM D 9379))-for liquids with a viscosity equal to or greater than 45 SUS at $100^{\circ} \mathrm{F}$ $\left(37.8^{\circ} \mathrm{C}\right)$, or that contain suspended solids. or that have a tendency to form a surface film under test: or

(iii) Setaflash Closed Tester (see American National Standard Method of Test for Flash Point by Setaflash Closed Tester (ASTM D 3278-78)).

Organic peroxides, which undergo utoaccelerating thermal decomposition. are excluded from any of the Mashpoint determination methods specified above.
"Hozardous chemical" means a chemical for which there is statistically significant evidence based on a! leasl one study conducled in accord ince with established scientific principl's that acute or chronic health effer is may occur in exposed employe's. The term "health hazard" includes c'remicals which are carcinogens, loxic or highly toxic agents. reproductive toxins. irritants, corrosives, sensitizers, hepatotoxins, nephrotoxins. neuroloxins, agents which act on the hematopoietic systems, and agents which damage the lungs, skin, eyes, or mucous membranes.

Appendices $A$ and $B$ of the Hazard Communication Standard (29 CFR 1910.1200 ) provide further guidance in defining the scope of health hazards and determining whether or not a chemical is to be considered hazardous for purposes of this standard.

"Laboratory" means a facility where the "laboratory use of hazardous chemicals" occurs. It is a workplace where relatively small quantities of hazardous chemicals are used on a nonproduction basis.

"Laboratory scale" means work with substances in which the containers used for reactions, transfers. and other handling of substances are designed to be easily and safely manipulated by one person. "Laboratory scale" excludes those workplaces whose function is to produce commercial quantities of materials.

"Laboratory-type hood" means a device located in a laboratory, enclosure on five sides with a moveable sash or fixed partial enclosed on the remaining side: constructed and maintained to draw air from the laboratory and to prevent or minimize the escape of air contaminants into the laboratory: and allows chemical manipulations to be conducted in the enclosure without insertion of any portion of the employee's body other than hands and arms.

Walk-in hoods with adjustable sashes meet the above definition provided that the sashes are adjusted during use so that the airflow and the exhaust of air contaminants are nol compromised and employees do not work inside the enclosure during the release of airborne hazardous chemicals.

"Laboralory use of hazardous chemicals" means handling or use of such chemicals in which all of the following condilions are mel

(i) Chemical manipulations are carried out on a "laboratory scale:"

(ii) Mulliple chemical procedures or chemicals are used: (iii) The procedures involved are not part of a production process, nor in any way simulate a production process: and

(iv) "Protective laboratory praclices and equipmenl" are available and in common use to minimize the potential for employee exposure to hazardous chemicals.

"Medical consultation" means a consultation which takes place between an employee and a licensed physician for the purpose of determining what medical examinations or procedures, if any, are appropriate in cases where a significant exposure to a hazardous chemical may have taken place.

"Organic peroxide" means an organic compound that contains the bivalent $-\mathrm{O}-\mathrm{O}$-structure and which may be considered to be a structural derivative of hydrogen peroxide where one or both of the hydrogen atoms has been replaced by an organic radical.

"Oxidizer" means a chemical other than a blasting agent or explosive as defined in $\$ 1910.109(a)$, that initiates or promotes combustion in other materials. thereby causing fire either of itself or through the release of oxygen or other gases.

"Physical hazard" means a chemical for which there is scientifically valid evidence that it is a combustible liquid. a compressed gas, explosive. flammable. an organic peroxide, an oxidizer. pyrophoric, unstable (reactive) or waterreactive.

"Protective laboratory practices and equipment" means those laboratory procedures, practices and equipment accepted by laboratory health and safety experts as effective. or that the employer can show to be effective. in minimizing the potential for employee exposure to hazardous chemicals.

"Reproductive toxins" means chemicals which affect the reproductive capabilities including chromosomal damage (mulations) and effects on fetuses (teratogenesis)

"Select carcinogen" means any substance which meets one of the following criteria:

(i) It is regulated by OSHA as a carcinogen: or

(ii) Il is listed under the category. "known to be carcinogens." in the Annual Report on Carcinogens published by the National Toxicology Program (NTP) (latest edition): or

(iii) It is lisled under Group 1 ("carcinogenic to humans") by the International Agency for Research on Cancer Monographs (IARC) llatest editions): or

(iv) Il is listed in either Group $2 A$ or 2B by IARC or under the callegory. "reasonably anlicipated lo be 
carcinogens" by NTP. and causes statistically significant tumor incidence in experimental animals in accordance with any of the following criteria:

(A) After inhalation exposure of 6-7 hours per day. 5 days per week. for a significant portion of a lifetime to dosages of less than $10 \mathrm{mg} / \mathrm{m}^{3}$ :

(B) After repeated skin application of less than $300(\mathrm{mg} / \mathrm{kg}$ of body weight) per week: or

(C) After oral dosages of less than 50 mg/kg of body weight per day.

"Unstoble (reactive)" means a chemical which is the pure state. or as produced or transported. will vigorously polymerize. decompose. condense. or will become self-reactive under conditions of shocks. pressure or temperature.

"Water-reoctive" means a chemical that reacts with water to release a gas that is either flammable or presents a health hazard.

(c) Permissible exposure limits. For laboratory uses of OSHA regulated substances. the employer shall assure that laboratory employees' exposures to such substances do not exceed the permissible exposure limits specified in 29 CFR part 1910. subpart Z.

(d) Employee exposure determination-11) Initial monitoring. The employer shall measure the employee's exposure to any substance regulated by standard which requires monitoring if there is reason to believe that exposure levels for that substance routinely exceed the action level (or in the absence of an action level. the PEL).

(2) Periodic monitoring. If the initial monitoring prescribed by paragraph (d)(1) of this section discloses employee exposure over the action level (or in the absence of an action level. the PEL). the employer shall immediately comply with the exposure monitoring provisions of the relevant standard.

(3) Termination of monitoring. Monitoring may be terminated in accordance with the relevant standard.

(4) Employee notification of monitoring results. The employer shall. within 15 working days after the receipt of any monitoring results, notify the employee of these results in writing either individually or by posting results in an appropriate location that is accessibie to employees.

(e) Chemical hygiene plan-General. (Appendix A of this section is nonmandatory but provides guidance to assist employers in the development of the Chemical Hygiene Plan.) (1) Where hazardous chemicals as defined by this standard are used in the workplace. the employer shall develop and carry ous the provisions of a wrillen Chemical Hygiene Plan which is: (i) Capable of protecting employees from health hazards associated with hazardous chemicals in that laboratory and

(ii) Capable of keeping exposures below the limits specified in paragraph (c) of this section.

(2) The Chemical Hygiene Plan shall be readily available to employees. employee representatives and. upon request. to the Assistant Secretary.

(3) The Chemical Hygiene Plan shall include each of the following elements and shall indicate specific measures that the employer will take to ensure laboratory employee protection:

(i) Standard operating procedures relevant 10 safety and health considerations to be followed when laboratory work involves the use of hazardous chemicals:

(ii) Criteria that the employer will use to determine and implement control mecsures to reduce employee exposure to hazardous chemicals including engineering controls. the use of personal protective equipment and hygiene practices: particular attention shall be given to the selection of control measures for chemicals that are known to be extremely hazardous:

(iii) A requirement that fume hoods and other protective equipment are functioning properly and specific measures that shall be taken to ensure proper and adequate performance of such equipment:

(iv) Provisions for employee information and training as prescribed in paragraph (f) of this section:

(v) The circumstances under which a particular laboratory operation. procedure or activity shall require prior approval from the employer or the employer's designee before implementation:

(vi) Provisions for medical consultation and medical examinations in accordance with paragraph ( 8 ) of this section:

(vii) Designation of personnel responsible for implementation of the Chemical Hygiene Plan including the assignment of a Chemical Hygiene Officer and. if appropriate. establishment of a Chemical Hygiene Committee: and

(viii) Provisions for additional employee protection for work with particularly hazardous substances. These include "select carcinogens." reproductive toxins and substances which have a high degree of acute loxicity. Specific consideration shall be given to the following provisions which shall be included where appropnate:

(A) Establishment of a designated area:
(B) Use of containment devices such as fume hoods or glove boxes:

(C) Procedures for safe removal of contaminated weste; and

(D) Decontamination procedures.

(4) The employer shall review and evaluate the effectiveness of the Chemical Hygiene Plen at least annually and update it as necessary.

(I) Employee information and training. (1) The employer shall provde employees with information and training to ensure that they are apprised of the hazards of chemicals present in their work area.

(2) Such information shall be provided at the time of an employee's initial assignment to a work area where hazardous chemicals are present and prior to assigmments involving new exposure situations. The frequency of refresher information and training shall be determined by the employer.

(3) Informotion. Employees shall be informed of:

(i) The contents of this standard and its appendices which shall be made available to employees;

(ii) The location and availability of the employer's Chemical Hygiene Plan:

(iii) The permissible exposure limits for OSHA regulated substances or recommended exposure limits for other hazardous chemicals where there is no applicable OSHA standard:

(iv) Signs and symptoms associsted with exposures to hazardous chemicals used in the laboratory: and

(v) The location and availability of known reference material on the hazards. safe handling. storage and disposal of hazardous chemicals found in the laboratory including. but not limited to. Material Safety Data Sheets received from the chemical supplier.

(4) Training. (i) Employee training shall include:

(A) Methods and observations that may be used to detect the presence or release of a hazardous chemical (such as monitoring conducted by the employer. continuous moxitoring devices. visual appearance or odor of hazardous chemicals when being released. etc.);

(B) The physical and health hazards of chemicals in the work ares: and

(C) The measures employees can rake to protect themselves from these hazards. including specific procedures the employer has implemented 10 protect employees from exposure to hazardous chemicals. such as appropriale work practices. emergency procedures. and personal protective equipment to be used. 
(ii) The employee shall be trained on the applicable details of the employer's written Chemical Hygiene Plan.

(B) Medical consultation and medical examinations. (1) The employer shall provide all employees who work with hazardous chemicals an opporlunity to receive medical attention, including any follow-up examinations which the examining physician determines to be necessary, under the following circumstances:

(i) Whenever an employee develops signs or symptoms asscciated with a hazardous chemical to which the employee may have been exposed in the laboratory, the employee shall be provided an opportunity to receive an appropriate medical examination.

(ii) Where exposure monitoring reveals an exposure level routinely above the action level (or in the absence of an action level, the PEL) for an OSHA regulated substance for which there are exposure monitoring and medical surveillance requirements, medical surveillance shall be established for the affected employee as prescribed by the particular standard.

(iii) Whenever an event takes place in the work area such as a spill, leak. explosion or other occurrence resulting in the likelihood of a hazardous exposure, the affected employee shall be provided an opportunity for a medical consultation. Such consultation shall be for the purpose of determining the need for a medical examination.

(2) All medical examinations and consultations shall be performed by or under the direct supervision of a licensed physician and shall be provided without cost to the employee. without loss of pay and at a reasonable time and place.

(3) Information provided to the physician. The employer shall provide the following information to the physician:

(i) The identity of the hazardous chemical(s) to which the employee may have been exposed;

(ii) A description of the conditions under which the exposure occurred including quantitative exposure data, if available; and

(iii) A description of the signs and symptoms of exposure that the employee is experiencing, if any.

(4) Physician's written opinion. (i) For examination or consultation required under this standard, the employer shall obtain a written opinion from the examining physician which shall include the following:

(A) Any recommendation for further medical follow-up;

(B) The results of the medical examination and any associated tests;
(C) Any medical condition which may be revealed in the course of the examination which may place the employee at increased risk as a resull of exposure to a hazardous chemical found in the workplace; and

(D) A statement that the employee has been informed by the physician of the results of the consultation or medical examination and any edical condition that may require further examination or treatment.

(ii) The written opinion shall not reveal specific findings of diagnoses unrelated to occupational exposure.

(h) Hazard identification. (1) With respect to labels and material safety data sheets:

(i) Employers shall ensure that labels on incoming containers of hazardous chemicals are not removed or defaced.

(ii) Employers shall maintain any material safety data sheets that are received with incoming shipments of hazardous chemicals, and ensure that they are readily accessible to laboratory employees.

(2) The following provisions shall apply to chemical substances developed in the laboratory:

(i) If the composition of the chemical substance which is produced exclusively for the laboratory's use is known, the employer shall determine if it is a hazardous chemical as defined in paragraph (b) of this section. If the chemical is determined to be hazardous, the employer shall provide appropriate training as required under paragraph (I) of this section.

(ii) If the chemical produced is a byproduct whose composition is not known, the employer shall assume that the substance is hazardous and shall implement paragraph (e) of this section.

(iii) If the chemical substance is produced for another user outside of the laboratory, the employer shall comply with the Hazard Communication Standard (29 CFR 1910.1200) including the requirements for preparation of material safety data sheets and labeling.

(i) Use of respirators. Where the use of respirators is necessary to maintain exposure below permissible exposure limits, the employer shall provide, at no cost to the employee, the proper respiratory equipment. Respirators shall be selected and used in accordance with the requirements of 29 CFR 1910.134.

(i) Recordkeeping. (1) The employer shall establish and maintain for each employee an accurate record of any measurements taken to monitor employee exposures and any medical consultation and examinations including tests or written opinions required by this standard.
(2) The employer shall assure that such records are kept, transferred, and made available in accordance with 29 CFR 1910.20.

(k) Dates-(1) Effective date. This section shall become effective May 1 1990.

(2) Start-up dates. (i) Employers shall have developed and implemented a written Chemical Hygiene Plan no later than January 31, 1991.

(ii) Paragraph (a)(2) of this section shall not take effect until the employer has developed and implemented a written Chemical Hygiene Plan.

(l) Appendices. The information contained in the appendices is not intended, by itself, to create any additional obligations not otherwise imposed or to detract from any existing obligation.

\section{Appendix A to 81810.1450 -National Research Council Recommendations Concerning Chemical Hygiene in Laboratories (Non-Mandatory) \\ Table of Contents \\ Foreword}

Corresponding Sections of the Standard and This Appendix

\section{A. General Principles}

1. Minimize all Chemical Exposures

2. Avoid Underestimation of Risk

3. Provide Adequate Ventilation

4. Institute a Chemical Hygiene Program

5. Observe the PELs and TLVs

\section{B. Responsibilities}

1. Chief Executive Officer

2. Supervisor of Administrative Unit

3. Chemical Hygiene Officer

4. Laboratory Supervisor

5. Project Director

6. Laboralory Worker

\section{The Laboratory Facility}
1. Design
2. Maintenance
3. Usage
4. Ventilation

D. Components of the Chemical Hygiene Plan

1. Basic Rules and Procedures

2. Chemical Procurement, Distribution, and

Storage

3. Environmental Monitoring

4. Housekeeping. Maintenance and Inspections

5. Medical Program

6. Personal Protective Apparel and

Equipment

7. Records

8. Signs and Labels

9. Spills and Accidents

10. Training and Information

11. Waste Disposa!

E. General Procedures for Working With Chemicals

1. General Rules for all Laboratory Work with Chemicals

2. Allergens and Embryotoxins 
3. Chemicals of Moderate Chronic or High

Acute Toxicily

4. Chemicals of High Chronic Toxicity

5. Animal Work with Chemicals of High

Chronic Toxicity

\section{F. Sofety Recommendations \\ G. Material Sofely Data Sheets}

\section{Foreword}

As guidance for each employer's development of an appropriate laboratory Chemical Hygiene Plan, the following nonmandatory recommendations are provided. They were extracted from "Prudent Practices for Handling Hazardous Chemicals in Laboratories" (referred to below as "Prudent Practices"). which was published in 1981 by the National Research Council and is available from the National Academy Press. 2101 Constitution Ave., NW. Washington DC 20418.

"Prudent Practices" is cited because of its wide distribution and acceptance and because of its preparation by members of the laboratory community through the sponsorship of the National Research Council. However, none of the recommendations given here will modify any requirements of the laboratory standard. This Appendix merely presents pertinent recommendations from "Prudent Practices", organized into a form convenient for quick reference during operation of a laboratory facility and during development and application of a Chemical Hygiene Plan. Users of this appendix should consult "Prudent Practices" for a more extended presentation and justification for each recommendation.

"Prudent Practices" deals with both safety and chemical hazards while the laboratory standard is concerned primarily with chemical hazards. Therefore, only those recommendations directed primarily toward control of toxic exposures are cited in this appendix. with the term "chemical hygiene" being substituted for the word "safety". However, since conditions producing or threatening physical injury often pose toxic risks as well, page references concerning . major categories of safety hazards in the laboratory are given in section F.

The recommendations from "Prudent Practices" have been paraphrased, combined. or otherwise reorganized. and headings have been added. However, their sense has not been changed.

Corresponding Sections of the Standard and this Appendix

The following lable is given for the convenience of those who are developing a Chemical Hygiene Plan which will satisfy the requirements of paragraph (e) of the standard. II indicales those sections of this appendix which are mosi perlinent to each of the sections of paragraph $(u)$ and relaled paragraphs.

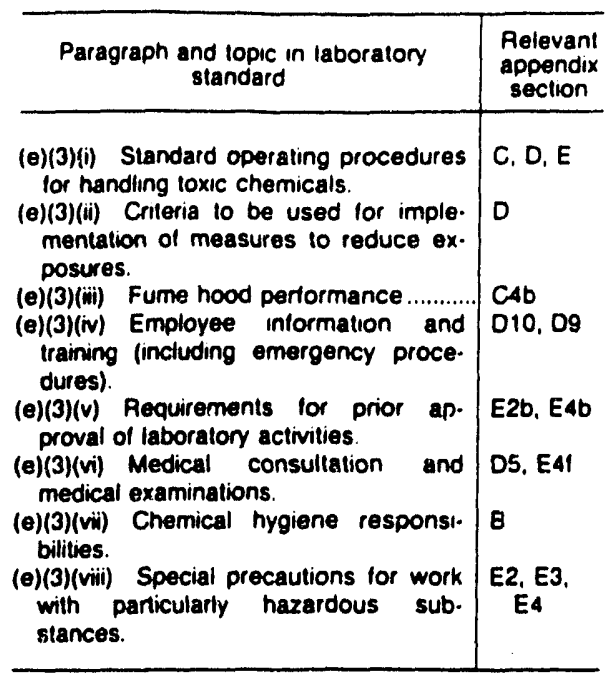

In this appendix, those recommendations directed primarily at administrators and supervisors are given in sections $A-D$. Those recommendations of primary concern to employees who are actually handling laboratory chemicals are given in section $\mathrm{E}$. (Reference to page numbers in "Prudent Practices" are given in parentheses.)

A. General Principles for Work with Laboratory Chemicals

In addition to the more detailed recommendations listed below in sections BE. "Prudent Practices" expresses certain general principles, including the following:

1. It is prudent to minimize all chemical exposures. Because few laboratory chemicals are without hazards, general precautions for handling all laboratory chemicals should be adopled, rather than specific guidelines for particular chemicals $(2,10)$. Skin contact with chemicals should be avoided as a cardinal rule (198).

2. Avoid underestimation of risk. Even for substances of no known significant hazard. exposure should be minimized; for work with substances which present special hazards. special precautions should be taken $(10.37$. 38). One should assume that any mixture will be more toxic than its most toxic component (30. 103) and that all substances of unknown toxicity are toxic (3. 34).

3. Provide adequate ventilation. The best way to prevent exposure to airborne substances is to prevent their escape into the working atmosphere by use of hoods and other ventilation devices $(32,198)$.

4. Institute a chemical hygiene program. A mandatory chemical hygiene program designed to minimize exposurps is needed: it should be a regular, continuing effort, not merely a standby or short-term activily 16 . 11). Its recommendations should be followed in academic teaching laboratories as well as by full-lime laboralory workers (13)

5. Observe the PELs. TLVs. The Permissible Exposure Limits of OSHA and the Threshold Limit Values of the American Conference of Covernmental Indusirial Hygienists should not be exceeded (13).
B. Chemical Hygiene Responsibilities

Responsibility for chemical hjgiene rests at all levels $(6,11,21)$ including the:

1. Chief executive officer, who has ultim responsibility for chemical hygiene within institution and must, with other

administrators, provide continuing support for institutional chemical hygiene $(7,11)$.

2. Supervisor of the department or other administrative unit. who is responsible for chemical hygiene in that unit (7).

3. Chemical hygiene officer(s), whose appointment is essential (7) and who must

(a) Work with administrators and other employees to develop and implement appropriate chemical hygiene policies and practices (7):

(b) Monitor procurement, use, and disposal of chemicals used in the lab (8):

(c) See that appropriate audits are maintained (8):

(d) Help project directors develop precautions and adequate facilities (10):

(e) Know the current legal requirements concerning regulated substances (50); and

(f) Seek ways to improve the chemical hygiene program $(8,11)$.

4. Laboratory supervisor, who has overall responsibility for chemical hygiene in the laboratory (21) including responsibility to:

(a) Ensure that workers know and follow the chemical hygiene rules, that protective equipment is available and in working order. and that appropriate training has been provided $(21,22)$;

(b) Provide regular, formal chemical hygiene and housekeeping inspections including routine inspections of emergency equipmsnt $(21,171)$ :

(c) Know the current legal requirements concerning regulated substances $(50,231)$;

(d) Determine the required levels of protective apparel and equipment (156, 160 , 162): and

(e) Ensure that facilities and training for use of any material being ordered are adequate (215).

5. Project director or director of other specific operation, who has primary responsibility for chemical hygiene procedures for that operation (7).

6. Laboratory worker, who is responsible for:

(a) Planning and conducting each operation in accordance with the institutional chemical hygiene procedures $(7,21,22,230)$; and

(b) Developing good personal chemical hygiene habits (22)

C. The Laboratory Facility

1. Design. The laboratory facility should have:

(a) An appropriate general ventilation system (see $\mathrm{C}_{4}$ below) with air intakes and exhausis located so as to avoid inlake of contaminated air (194):

(b) Adequate, well-ventilated slockrooms/ storerooms (218, 219):

(c) Laboratory hoods and sinks (12, 162):

(d) Other safely equipment including eyewash fountains and drench showers (16 169): and

(e) Arrangements for waste disposal 112 
2. Maintenance. Chemical-hygiene-related equipment (hoods, incinerator, etc.) should undergo continuing appraisal and be modified if inadequate $(11,12)$.

3. Usage. The work conducted (10) and its scale (12) must be appropriate to the physicial facilities available and, especially. to the quality of ventilation (13).

4. Ventilation-(a) General laboratory ventilation. This system should: Provide a source of air for breathing and for input to local ventilation devices (199); it should not be relied on for protection from toxic substances released into the laboratory (198): ensure that laboratory air is continually replaced, preventing increase of air concentrations of toxic substances during the working day (194); direct air flow into the laboratory from non-laboratory areas and out to the exterior of the building (194)

(b) Hoods. A laboratory hood with 2.5 linear feet of hood space per person should be provided for every 2 workers if they spend most of their time working with chemicals (199); wach hood should have a continuous monitoring device to allow convenient confirmation of adequate hood performance before use $(200,209)$. If this is not possible. work with substances of unknown toxicity should be avoided (13) or other types of local ventilation devices should be provided (199). See pp. 201-206 for a discussion of hood design, construction, and evaluation.

(c) Other local ventilation devices. Ventilated storage cabinets, canopy hoods. snorkels, etc. should be provided as needed 199). Each canopy hood and snorkel should have a separate exhaust duct (207)

(d) Special ventilation areas. Exhaust air from glove boxes and isolation rooms should be passed through acrubbers or other treatment before release into the regular exhaust system (208). Cold rooms and warm rooms should have provisions for rapid escape and for escape in the event of electrical failure (209).

(e) Modifications. Any aiteration of the ventilation system should be made only if thorough testing indicates that worker protection from airborne toxic substances will continue $: 2$ be adequate $(12,193,204)$

(I) Performance. Rate: 4-12 room air chenges/hour is normally adequate general ventilation if local exhaust systems such as hoods are used as the primary method of control (194).

(8) Quality. General air flow should not be turbulent and should be relatively uniform throughout the laboratory, with no high velocity or static areas (194, 195); airnow into and within the hood should not be excessively turbulent (200); hood face velocity should be adequate (typically 60-100 Ifm) (200. 204).

(h) Evaluation. Quality and quantity of ventilation should be evaluated on installation (202), regularly monitored (at least every 3 months) $(6,12,14,195)$, and eevaluated whenever a change in local entilation devices is made $(12,195,207)$. See pp. 195-198 for methods of evaluation and for calculation of estimated airborne contaminant concentrations.
D. Components of the Chemical Hygiene Plan

1. Basic Rules and Procedures

(Recommendations for these are given in section $\mathrm{E}$, below)

2. Chemical Procurement, Distribution, and Storage

(a) Procurement. Before a substance is received, information on proper handling. storage, and disposal should be known to those who will be involved $(215,216)$. No container should be accepted without an adequate identifying label (216). Preferably. all substances should be received in a central location (216)

(b) Stockrooms/storerooms. Toxic substances should be segregated in a wellidentified area with local exhaust ventilation (221). Chemicals which are highly toxic (227) or other chemicals whose containers have been opened should be in unbreakable secondary containers (219). Stored chemicals should be examined periodically (at least annually) for replacement. deterioration, and container integrity $(218-x, 8)$.

Stockrooms/storerooms should not be used as preparation or repackaging areas. should be open during normal working hours, and should be controlled by one person (219).

(c) Distribution. When chemicals are hand carried, the container should be placed in an outside container or bucket. Freight-only elevators should be used if possible (223).

(d) Laboratory storage. Amounts permitted should be as amall as prectical. Storage on bench tops and in hoods is inadvisable. Exposure to heat or direct sunlight should be avoided. Periodic inventories should be conducted, with unneeded items being discardeci or returned to the storeroom/ stockroom $(225-6,229)$.

\section{Environmental Monitoring}

Regular instrumental monitoring of airborne concentrations is not usually justified or practical in laboratories but may be appropriate when testing or redesigning hoods or other ventilation devices (12) or when a highly toxic eubstance is stored or used regularly (e.g., 3 times/week) (13).

4. Housekeeping, Maintenance, and Inspections

(a) Cleaning. Floors should be cleaned regularly (24).

(b) Inspections. Formal housekeeping and chemical hygiene inspections should be held. at least quarterly $(6,21)$ for units which have frequent pesonnel changes and semiannually for others: informal inspections should be continual (21)

(c) Maintenance. Eye wash fountains should be inspected at intervals of not less than 3 months (8). Respirators for routine use should be inspected periodically by the laboratory supervisor (169). Safety showers should be tested routinely (169). Other safety equipment should be inspected regularly. (e.8., every 3-6 months) $(6,24,171)$. Procedures to prevent restarting of out-of. service equipment should be established (25).

(d) Passageways. Slairways and hallways should not be used as storage areas (24). Access to exits, emergency equipment, and utility controls should never be blocked (24)
5. Medical Program

(a) Compliance with regulations. Regular medical surveillance should be established to the extent required by regulations (12).

(b) Routine surveillance. Anyone whose work involves regular and frequent handling of toxicologically significant quantities of a chemical should consult a qualified physician to determine on an individual basis whether a regular schedule of medical surveillance is desirable $(11,50)$.

(c) First aid. Personnel trained in first aid should be available during working hours and an emergency roorn with medical personnel should be nearby (173). See pp. 176-178 for description of some emergency first aid procedures.

\section{Protective Apparel and Equipment}

These should include for each laboratory:

(a) Protective apparel compatible with the required degree of protection for substances being handled (158-161);

(b) An easily accessible drench-type safety shower $(162,169)$;

(c) An eyewash fountain (162):

(d) A fire extinguisher (162-164);

(e) Respiratory protection (164-9), fire

alarm and telephone for emergency use (162)

should be available nearby; and

(f) Other items designated by the

laboratory supervisor $(156,160)$.

7. Records

(a) Accident records should be written and retained (174).

(b) Chemical Hygiene Plan records should document that the facilities and precautions were compatible with current knowledge and regulations (7).

(c) Inventory and usage records for high risk substances should be kept as specified in sections E3e below.

(d) Medical records should be retained by the institution in accordance with the requirements of state and federal regulations (12).

\section{Signs and Labels}

Prominent signs and labels of the following types should be posted:

(a) Emergency telephone numbers of emergency personnel/facilities, supervisors, and laboratory workers (28);

(b) Identity labels, showing contents of containers (including waste receptacles) and associated hazards $(27,48)$

(c) Location signs for safely showers. eyewash stations, other safety and first aid equipment, exits (27) and areas where food and beverage consumption and storage are permitted (24); and

(d) Warnings at areas or equipment where special or unusual hazards exist (27).

9. Spills and Accidents

(a) A written emergency plan should be established and cornmunicated to all personnel: it should include procedures for ventilation failure (200), evacuation, medica care, reporting, and drills (172).

(b) There should be an alarm system to alert people in all parts of the facility including isolation areas such as cold rooms (172) 
(c) A spill control policy should be developed and should include consicieration of prevention. containment. cleanup. and reporting (175).

(d) All accidents or near accidents should be carefully analyzed with the results distributed to all who might benefit (8.28). 10. Information and Training Progras

(a) Aim: To assure that all individuals at risk are adequately informed about the work in the laboralory. its risks. and what to do if an accident occurs (5. 15).

(b) Emergency and Personal Protection Training: Every laboratory worker should know the location and proper use of avaliable protective apparel and equipment (154. 169).

Some of the full-time personnel of the laboratory should be trained in the proper use of emergency equipment and procedures (6).

Such training as well as first aid instruction should be available to (154) and encouraged for (176) everyone who might need it.

(c) Receiving and stockroom/storeroom persornel should know about hazardis. handling equipment. protective apparel. and releveni regulations (217).

(d) Frequency of Training: The traning and education program should be a regular. continuing activity - not simply an annual presentation (25).

(e) Literature/Consultation: Literature and consulting advice concerning chemical hygiene should be readily available to laboratory personnel. who should be encouraged to use these information resources (14).

12. Waste Disposol Progrom.

(a) Aim: To assure that minimal harm to people. other organisms. and the environment will result from the disposal of waste laboratory chemicals (5).

(b) Content (14.232, 233. 240): The waste diaposal program should specify how waste is to be collected. segregated. stored. and transported and include consideration of what materials can be incinerated. Transport from the institution must be in eccordance with DOT regulations (24)

(c) Discarding Chemical Slocks: Unlabeled containers of chemicals and solutions should undergo prompt disposal: if partially used. they should not be opened $(24.27)$.

Before a worker's employment in the laboratory ends. chemicals for which that person was responsible should be discarded or retumed to storage (228).

(d) Frequency of Disposal: Waste should be removed from laboratories to central waste storage srea at least once per week and from the central waste storage aree at regulas intervals (14).

(e) Method of Disposal: Incineration in an environmentally acceptable manuer is the mosi practical disposal method for combustible laboratory waste (14. 236. 241).

Indiscriminate disposal by pouring waste chemicals down the drain (14. 231. 242) or adding them to mixed refuse for landrill burial is unacceptable (14)

Hoods should not be used as a means of disposal for volatile chemicals $(40.200)$.

Disposal by recycling $(233.243)$ or chemical deconiamination (40. 230) should be used when possible.
E. Bosic Rules and Proceciures ior Working ivith Chemicals

The Chemical Hygiene Plan should require that laboratory workers know and follow its rules and procedures. In addition to the procedures of the sub programs mentioned above. these should include the rules listed below.

1. General Rules

The following should be used for essentially all laboratory work with chemicals:

(a) Accidents and spills-Eye Contact: Prompily flush eyes with water for a prolonged period (15 minutes) and seek medical attention (33. 172).

Ingestion: Encourage the victim to drink large amounts of water (178).

Skin Contact: Promptly flush the affected area with water (33.172 178) and remove any contaminated clothing (172. 178). Uf symptoms persist after weshing, seek medical attention (33).

Clean-up. Promptly clean up spills. using appropriate protective apparel and equipment and proper disposal (2433). See pp. 233-237 for specific clean-up recommendations.

(b) A voidance of "routine" exposure: Develop and encourage safe habits (23): avoid unnecessary exposure to chemicals by any route (23):

Do not smell or taste chemicals (32). Vent epparatus which may discharge toxic chemicals (vacuum pumps. distillation columns. etc) into local exhaust devices (189).

Inspect gloves (157) and test glove boxes

(208) before use.

Do not allow release of toxic substances in cold rooms and warm rooms. since these have contained recirculated atmospheres (209).

(c) Choice of chemicals: Use only those chemicals for which the quality of the available venulation system is appropriate (13).

(d) Eating. smoking. elc.: Avoid eating. drinking. smcking. gum chewing. or application of cosmetics in areas where laboratory chemicals are present $(22,24,32$ 40): wash hands before conducting these activities $(23,24)$.

Avoid atorage. handling or consumption of lood or beverages in storage areas. refrigeratorn. glassware or utensils which are also used for laboralory operationa $123,24$. 228).

(e) Equipment and glasswore: Handle and store laboratory glaseware with care to avoid damage: do not use damaged glassware (25). Use extra care with Dewar hasks and other evacuated glass apparatus: shield or wrap them to contain chemicals and fragments should implosion occur (25). Use equipment only for its designed purpose $(23.26)$.

(I) Exiting: Wash areas of exposed skin

well before leaving the laboratory (23)

(8) Horseplay: Avoid praclical jokes or other behavior which mighi confuse. slartle or distract another worker (23).

(h) Mouth suction: Do nol use mouth suction for pipeting or starting a siphon 123. 32).

(i) Personal apporel: Confine long hair and loose clothing (23. 258). Wear shoes at all times in the laboratory but do not wear sandals. perforated shoes. or sneakers (158

(i) Personal housekeeping: Keep the wort area clean and uncluttered. with chemicals and equipment being property labeled and stored: clean up the work area on completion of an operation or at the end of each day (24).

(k) Personal protection: Assure that epproprate eye protection (154-156) is wom by all persons. including visitors. where chemicals are stored or handled $(22$ 23. 33. 154).

Wear appropriate gloves when the potential for contact with toxic materials exists (157): inspect the gloves before each use. wash them before removal. and replace them periodically (15\%). (A table of resistance to chemicals of common glove matenals is given p. 159).

Use appropriate (164-168) respiratory equipment when air contaminant concentrations are not sufficiently restncted by enguneering controls (164-5). inspecting the respirator before use (169).

Use any other protective and emergency apparel and equipment as appropnate 122. 157-162).

Avoid use of contact lenses in the laboratory uniess necessary: if they are used. inform supervisor so special precautions can be laken (155).

Remove leboratory coats immediately on significant contamination (161).

(1) Plonning: Seek information and advice about hazards (7). plan approprinte protecti procedures. and plen positioning of equipment before beginning any new operation (22, 23).

(m) Unattended operations: Leave lights on place en appropriate sign on the door. and provide for containment of toxic substunces in the event of failure of a utility service (such as cooling water) to an unattended operation (27. 128).

(n) Use of hood: Use the hood for operstions which might result in release of toxic chemical vapors or dust (198-0).

As a rule of thumb. use a hood or other local ventilation device when wortins with any appreciably volatile substance with a TIV of less than $50 \mathrm{ppm}$ (13).

Confirm adequate hood performance before use: keep hood closed at all times except when adjustments within the hood are being made (200): keep materialo stored in hoods to minimum and do not allow them to block vents or air flow (200).

Leave the hood "on" when it is not in active use if toxic substances are stored in it or if it is uncertain whether adequate general laboratory ventilation will be maintained when it is "off" (200).

(0) Vigilance: Be alert to ungafe conditions and see that they are corrected when detected (22).

(p) Woste disposal: Assure that the plan for each laboralory operation includes plans and training for waste disposal (230).

Deposit chemical waste in appropriately labeled receptacles and follow all other waste disposal procedures of the Chemical Hygiene Plan (22.24).

Do not discharge to the sewer concentrated acids or bases (231): highly toxic. malodorous. or lachrimatory substances 
(231); or any substances which might interfere with the hiological activity of waste water treatment plants. create fire or explosion hazards. cause structural damage or obstruct flow (242).

(q) Working alone: Avoid working alone in a building: do not work alone in a laboratory if the procedures being conducted are hazardous (28).

2. Working with Allergens and Embryotoxins

(a) Allergens (examples: diazomethane, isocyanates, bichromates): Wear suitable gloves to prevent hand contact with allergens or substances of unknown allergenic activity (35).

(b) Embryotoxins (34-5) (examples: organomercurials. lead compounds. formamide): If you are a woman of childbearing age, handle these substances only in a hood whose satisfactory performance has been confirmed, using appropriate protective apparel (especially gloves) to prevent skin contact.

Review each use of these materials with the research supervisor and review continuing uses annually or whenever a procedural change is made.

Store these substances, properly labeled. in an adequately ventilated area in an unbreakable secondary container.

Notify supervisors of all incidents of exposure or spills: consult a qualified physician when appropriate.

3. Work with Chemicals of Moderate Chronic or High Acute Toxicity

Examples: diisopropylflurophosphate (41). hydrofluoric acid (43), hydrogen cyanide (45). Supplemental rules to be followed in addition to those mentioned above (Procedure B of "Prudent Practices", pp. 3941):

(a) Aim: To minimize exposure to these toxic substances by any route using all reasonable precautions (39).

(b) Applicability: These precautions are appropriate for substances with moderate chronic or high acute toxicity used in significant quantities (39).

(c) Location: Use and store these substances only in areas of restricted access with special warning signs $(40,229)$.

Always use a hood (previously evaluated to confirm adequate performance with a face velocity of at least 60 linear feet per minute) (40) or other containment device for procedures which may result in the generation of aerosols or vapors containing the substance (39): trap released vapors to prevent their discharge with the hood exhaust (40).

(d) Personal protection: Always avoid skin contacl by use of gloves and long sleeves (and other prolective apparel as appropriate) (39). Always wash hands and arms immediately after working with these materials (40).

(e) Records: Maintain records of the amounts of these materials on hand. amounts used. and the names of the workers involved (40. 229).

(n) Prevention of spills and accidents: Be repared for accidents and spills (4i)

Assure that at least 2 people are present at all times if a compound in use is highly toxic or of unknown loxicity (39).
Store breakable containers of these substances in chemically resistant trays: also work and mount apparatus above such trays or cover work and storage surfaces with removable, absorbent, plastic backed paper (40).

If a major spill occurs outside the hood. evacuate the area: assure that cleanup personnel wear suitable protective apparel and equipment (41).

(8) Waste: Thoroughly decontaminate or incinerate contaminated clothing or shoes (41). If possible, chemically decontaminate by chemical conversion (40).

Store contaminated waste in closed. suitably labeled, impervious containers (for liquids, in glass or plastic bottles half-filled with vermiculite) (40)

4. Work with Chemicals of High Chronic Toxicity

(Examples: dimethylmercury and nickel carbonyl (48), benzo-a-pyrene (51), $\mathrm{N}$ nitrosodiethylamine (54), other human carcinogens or substances with high carcinogenic potency in animals (38).

Further supplemental rules to be followed. in addition to all these mentioned above, for work with substances of known high chronic toxicity (in quantities above a few milligrams to a few grams, depending on the substance) (47). (Procedure A of "Prudent Practices" pp. 47-50).

(a) Access: Conduct all transfers and work with these substances in a "controlled area": a restricted access hood, glove box, or portion of a lab, designated for use of highly toxic substances, for which all people with access are aware of the substances being used and necessary precautions (48).

(b) Approvals: Prepare a plan for use and disposal of these materials and obtain the approval of the laboratory supervisor (48).

(c) Non-contamination/Decontamination: Protect vacuum pumps against contamination by scrubbers or HEPA filters and vent them into the hood (49). Decontaminate vacuum pumps or other contaminated equipment. including glassware, in the hood before removing them from the controlled area (49. 50).

Decontaminate the controlled area before normal work is resumed there $(50)$.

(d) Exiting: On leaving a controlled area, remove any protective apparel (placing it in an appropriate, labeled container) and thoroughly wash hands, forearms, face, and neck (49).

(e) Housekeeping: Use a wet mop or a vacuum cleaner equipped with a HEPA filter instead of dry sweeping if the toxic substance was a dry powder (50).

(f) Medical surveillance: If using toxicologically significant quantities of such a substance on a regular basis (e.g., 3 times per week), consult a qualified physician concerning desirability of regular medical surveillance (50)

(8) Records: Keep accurate records of the amounts of these substances stored (229) and used, the dates of use, and names of users (48)

(h) Signs and labels: Assure that the controlled area is conspicuously marked with warning and restricled access signs (49) and that all containers of ihese substances are appropriately labeled with identity and warning labels (48).

(i) Spills: Assure that contingency plans. equipment, and materials to minimize exposures of people and property in case of accident are available (233-4).

(i) Storage: Store containers of these chemicals only in a ventilated, limited access $(48,227,229)$ area in appropriately labeled. unbreakable, chemically resistant, secondary containers $(48,229)$.

(k) Glove boxes: For a negative pressure glove box, ventilation rate must be at least 2 volume changes/hour and pressure at least 0.5 inches of water (48). For a positive pressure glove box. thoroughly check for leaks before each use (49). In either case, trap the exit gases or filter them through a HIEPA filter and then release them into the hood (49).

(1) Waste: Use chemical decontamination whenever possible; ensure that containers of contaminated waste (including washings from contaminated flasks) are transferred from the controlled area in a secondary container under the supervision of authorized personnel $(49,50,233)$

5. Animal Work with Chemicals of High Chronic Toxicity

(a) Access: For large scale studies, special facilities with restricted access are preferable (56).

(b) Administration of the toxic substance: When possible, administer the substance by injection or gavage instead of in the diet. If administration is in the diet, use a caging system under negative pressure or under laminar air flow directed toward HEPA filter (56).

(c) Aerosol suppression: Devise procedures which minimize formation and dispersal of contaminated aerosols, including those from food, urine, and feces (e.g., use HEPA filtered vacuum equipment for cleaning, moisten contaminated bedding before removal from the cage, mix diets in closed containers in a hood) $(55,56)$.

(d) Personal protection: When working in the animal room, wear plastic or rubber gloves, fully buttoned laboratory coat or jumpsuit and, if needed because of incomplete suppression of aerosols, other apparel and equipment (shoe and head coverings, respirator) (56).

(e) Waste disposal: Dispose of contaminated animal lissues and excreta by incineration if the available incinerator can convert the contaminant to non-loxic products (238): otherwise, package the waste appropriately for burial in an EPA-approved site (239).

\section{F. Safety Recommendations}

The above recommendations from "Prudent Practices" do not include those which are directed primarily toward prevention of physical injury rather than toxic exposure. However, failure of precautions against injury will often have the secondary effect of causing toxic exposures. Therefore, we list below page references for recommendations concerning some of the major categories of safety hazards which also have implications for chemical hygiene:

1. Corrosive agents: (35-6) 
2. Electncally powered laboratory apparatus: (179-02)

3. Fires. explosions: (26. 57-74, 162-4. 174-5. 219-20. 226-71

4. Low temperature procedures: (26. 88)

5. Pressurized and vacuum operations (including use of compressed gas cylinders): (27. 75-101)

\section{Molerial Safety Dato Sheets}

Material safety data sheets are presented in "Prudent Practices" for the chemicais listed below. (Astensks denote that comprehensive material safety data sheets are provided).

- Acelyl peroxide (105)

- Acrolein (106)

- Acrylonilrile (107)

Ammonis (anbydrous) (91)

- Ariline (109)

- Benzere (110)

- Benzolalpyrene (112)

- Bis(chloromethyl) ether (113)

Boron enchloride (91)

Boron trifluoride (92)

Bromine (114)

-Tert-butyl hydroperoxide (148)

- Carbon disulfide (116)

Carbon monoxide (92)

-Carbon tetrachlonde (118)

- Chlorine (119)

Chlorine trifluoride (94)

Chloroform (121)

Chloromethane (93)

- Diethyl ether (122)

Dilisopropyl Auorophosphate (41)

-Dimethylformamide (123)

- Dimethyl sulfate (125)

- Dioxane (126)

Ethylene dibromide (128)

- Fluorine (95)

-Formaldehyde (130)

'Hydrazine and salis (132)

Hydrolluoric acid (43)

Hydrogen bromide (98)

Hydrogen chloride (98)

-Hydrogen cyanide (133)

-Hydrogen sulfide (135)

Mercury and compounds (52)

- Methanol (137)

- Morpholine (138)

- Nickel carbonyl (99)

- Nitrobenzene (139)

Nitrogen dioxide $(100)$

$N$-nitrosodiethylamine (54)

- Perncetic acid (141)

- Phenol (142)

- Phosgene (143)

- Pyridine (144)

- Sodium azide (145)

- Sodlum cyanide (147)

Sulfur dioxide (101)

- Trichloroethylene (149)

-Vinyl chloride (150)

Appeadix B to I 1970.1450 -Reforences (Non-Mandatory)

The following references are provided to assiat the employer in the development of a Chemical Hygiene Plan. The materials listed below are offered as non-mandatory guidance. References listed here do not imply specific endorsement of a book. opınion. technique. policy or a specific solution for a safety or heslth problem. Other references not listed here may better meet the needs of a specific laboratory. (a) Materials for the development of the Chemical Hygiene Plan:

1. American Chemical Sociely. Safety in Academic Chemistry Laboratones. 4th edition. 1985.

2. Fawcetl. H.H. and W. S. Wood. Safety and Accident Prevention in Chemical Operations. 2nd edition. Wiley-Interscience. New York 1982

3. Flury. Patricia A. Environmental Health and Safety in the Hospital Laboratory.

Charles C. Thomas Publisher. Springfield IL 1978.

3. Green. Michael $E$ and Turk. Amos. Safety in Working with Chemicals.

Maconillan Publishing Co. NY. 1978.

5. Kaufrnar. James A. Laboratory Safety Guidelines. Dow Chemical Co.. Box 1713.

Midland. Ml 48640. 1977.

6. National Institutes of Health. NLH

Guidelines for the Laboratory use of

Chemical Carcinogens. NIH Pub. No. 81-2385.

GPO. Washington. DC 20402. 1981.

7. National Research Council. Prudent Practices for Disposal of Chemicals from Laboratories. National Acadenny Press. Washington. DC. 1983

8. National Research Council. Prudent Practices for Handling Hazardous Chemicala in Laboratories. National Academy Press. Washington. DC 1981.

9. Renfrew. Malcolm. Ed. Safety in the Chemical Laboratory. Vol. IV. I. Chem. Ed. American Chemical Society. Easlon. PA. 1901.

10. Steere. Norman V. Ed. Safety in the Chemical Laboratory. J. Chem. Ed. American Chemical Society. Easlon. PA. 18042 Vol. L. 1967. Vol. II. 1971. Vol. III 1974.

11. Steere. Norman V.. Handbook of Laboratory Safety. the Chemical Rubber Company Cleveland. OH. 1971.

12. Young. lay A.. Ed.. Improving Safety in the Chemical Laboratory. John Wiley \& Sons. Inc. New York. 1987.

(b) Hazardous Substances Information: 1. American Conference of Govermmental Industrial Hygienists. Threshold Limit Values for Chemical Substances and Physical Agents in the Workroom Environment with intended Changes. P.O. Box 1937 Cincinnati. OH 45201 (latesl edition).

2. Annual Report on Carcinogens. National Toxicology Program U.S. Department of Health and Human Services. Public Health Service. U.S. Government Printing Office. Washington. DC (latest edition).

3. Best Company. Bes! Safety Directory. Vols. I and II. Oldwick. N.J., 1981.

4. Bretherick. L. Handbook of Reactive Chemical Hazards. 2nd edition. Butterworths. London. 1979.

5. Bretherick. L. Hazards in the Chemical Laboratory. 3rd edition. Royal Sociely of Chemistry. London. 1986.

6. Code of Federal Regulations. 29 CFR part 1910 subpart 2. U.S. Govt. Printing Office. Washington. DC 20402 (latest edition).
7. IARC Monographs on the Evaluation of the Carcinogenic Risk of Chemicals 10 . Wan World Health Organization Publications Center. 49 Sheridan Avenue. Albani. New York 12210 (latest editions).

8. NIOSH/OSHA Pocket Guide to Chemicai

Hazards. NIOSH Pub. No. 85-114. U.S.

Government Printing Office. Washington. DC. 1385 (or latest edition).

9. Occupational Health Guidelines.

NIOSH/OSHA NIOSH Pub. No. 81-123 U.S

Govemment Printing Office. Washington. DC. 1981.

10. Patty. F.A.. Industral Hygiene and

Toxicology. John Wiley \& Sons. Inc.. New

York. NY (Five Volumes).

11. Registry of Toxic Effects of Chemical

Substances. U.S. Department of Health and

Human Services. Public Health Sernce.

Centers for Disease Control. National

Institute for Occupationsl Safety and Health.

Revised Annually. for sale from

Superintendent of Documents U.S. Govt.

Printing Office. Washington. DC 20402.

12. The Merck Index: An Encyclopedia of

Chemicals and Drugs. Merck and Cornpany

Inc. Rahway. N.].. 1976 (or latest edition).

13. Sax. N.l. Dangerous Properties of

Industrial Matenals. 3th edition. Van

Nostrand Reinhold. NY.. 1979.

14. Sittig. Marshall. Handbook of Toxic and

Hazardous Chemicals. Noyes Publications.

Park Ridge. N|. 1981.

(c) Information on Ventilation:

1. American Conference of Governmental

Industrial Hygienists Industrial Ventilation 10th edition Lansing. Ml. 1980

2. American National Standards Institute. Ine American National Standards

Fundamentals Governing the Design and

Operation of Local Exhaust Sustems AVSI 2

9.2-1979 American National Standards

Institute. N.Y. 1979.

3. Imad. A.P. and Watson. C.L Ventilation

Index: An Easy Way to Decide about

Hazardous Liquids. Professional Safety pp

15-18. April 1980.

4. National Fire Protection Association.

Fire Protection for Laboratories Using

Chemicals NFPA-45. 1982

Safety Standard for Laboratories in Health

Related Institutions. NFPA. 56C. 1980.

Fire Protection Guide on Hazandous

Materials. 7th edition. 1978.

National Fire Protection Associstion.

Batterymarch Park. Quincy. MA 02269.

5. Scientific Apparatus Makers Association (SAMA). Standard for Laboratory Fume

Hoods. SAMA L77-1980. 1101 16ih Street.

NW. Washington. DC 20036.

(d) Informatuon on Availability of

Referenced Material:

1. American National Standards Institute

(ANSI). 1430 Broadway. New York. NY 10018.

2. American Society for Testing and

Materials (ASTM). 1916 Race Street.

Philadelphia. PA 19103.

(Approved by the Office of Management and Budget under control number 1216-0131

[FR Doc. 90-1717 Filed 1-30-90: 8:45 am) oluwa coot astarm 


\section{Appendix 3}

\section{Glossary of Terms}

Action Level

acute effect

acute exposure

acute toxicity

aerosol

anesthetic

ANSI

asphyxiant

carcinogen

cardiac
A concentration designated in 29 CFR Part 1910 for a specific substance, calculated as an eight (8)-hour time-weighted average, that initiates certain required activities such as exposure monitoring and medical surveillance.

Symptom of exposure to a hazardous material that soon appears after a short-term exposure, coming quickly to a crisis.

A single, brief exposure to a large dose of a toxic substance. Adverse health effects are evident soon after exposure.

Adverse biological effects of a single dose of a toxic agent.

A suspension of fine solid or liquid particles in air (e.g., paint spray, mist, fog).

A chemical that causes drowsiness. Large doses of anesthetic chemicals can cause unconsciousness, coma, and death.

American National Standards Institute. This privately funded, voluntary organization develops standards for the safe design and operation of equipment and safe practices or procedures for industry.

A chemical vapor or gas that replaces air and can, thereby, cause death by suffocation. Asphyxiants are especially hazardous in confined spaces.

A chemical or physical agent that is known to cause cancer in humans or is thought possibly to cause cancer, based on evidence from experimental animals.

Refers to the heart. 
CAS Number

$\propto$

Ceiling Limit

central nervous system

chemical family

Chemical Hygiene Officer

Chemical Hygiene and Safety Plan

chronic exposure

chronic effect

chronic toxicity
Chemical Abstract Service registry number, which is used to identify a specific chemical.

Cubic centimeter. A metric-system volume measurement equal to a milliliter (ml). One quart is about $946 \mathrm{cc}(946 \mathrm{ml})$.

The maximum allowable exposure limit for an airborne chemical, which is not to be exceeded even momentarily. See also PEL and TLV.

The part of the body made up of the brain, spinal cord, and nerves.

Chemicals with similar structural characteristics are grouped into a chemical family (e.g., ketones, alcohols, hydrocarbons).

A person designated by the employer who is qualified by training or experience, to provide technical guidance in the development and implementation of the provisions of the Chemical Hygiene \& Safety Plan.

A written program developed and implemented by the employer which sets forth procedures, equipment, personal protective equipment and work practices that are capable of protecting employees from the health hazards presented by hazardous chemicals used in the particular workplace.

Repeated exposure or contact with a toxic substance over a long period. Adverse biological effects from chronic exposure develop slowly, last a long time, and frequently recur.

Symptom of exposure to a hazardous material that develops slowly after many exposures or that recurs often.

Adverse biological effect of repeated doses or longterm exposure to a toxic agent. 
combustible

combustible gas

combustible liquid

compressed gas

concentration

corrosive

decomposition
Able to catch on fire and burn. A liquid that will burn is called a "combustible liquid." Nonliquid substances that will burn, such as wood and paper, are called "ordinary combustibles." (See flammables)

(i) A gas or mixture of gases having, in a container, an absolute pressure exceeding 40 psi at $70 \circ \mathrm{F}\left(21.1^{\circ} \mathrm{C}\right)$; or

(ii) A gas or mixture of gases having, in a container, an absolute pressure exceeding $104 \mathrm{psi}$ at $130^{\circ} \mathrm{F}\left(54.4^{\circ} \mathrm{C}\right)$ regardless of the pressure at $70^{\circ} \mathrm{F}$ $\left(21.1^{\circ} \mathrm{C}\right)$ : or

(iii) A liquid having a vapor pressure exceeding 40 psi at $100^{\circ} \mathrm{F}\left(37.8^{\circ} \mathrm{C}\right)$ as determined by ASTM D323-72.

Any liquid having a flashpoint at or above $100^{\circ} \mathrm{F}$ but below 200 \% .

A gas or mixture of gases in a container having an absolute pressure of 40 or more psi at room temperature.

The relative amount of a given substance present when mixed with another substance(s). Concentration is often expressed as parts per million (ppm), percent, or weight per unit volume, e.g., milligrams/cubic meter $\left(\mathrm{mg} / \mathrm{m}^{3}\right)$.

A chemical that causes visible destruction of, or irreversible changes in living tissue by chemical action at the site of contact, or that has a severe corrosion rate on structural materials.

The breakdown of a material into a simpler compound by chemical reaction, decay, heat, or other process. 
density

dermal

dermatitis

designated area

dose

epidemiology

explosive

flammable

flashpoint
The mass of a substance per unit volume. The density of a liquid substance is usually compared to water, which has a density of 1 ; the density of a gas is usually compared to air. Substances that float on water have densities of less than 1; substances that sink in water have densities greater than 1.

Refers to skin.

An inflammation of the skin, which can be caused by irritation (chemical, physical, or mechanical) or allergic reaction.

An area that may be used for work with "select carcinogens, " reproductive toxins or substances which have a high degree of acute toxicity. A designated area may be the entire laboratory, an area of a laboratory or a device such as a laboratory hood.

The amount of a substance received during exposure.

The branch of medical science that deals with the incidence, distribution, and control of disease in a population.

A chemical that causes a sudden, almost instantaneous release of pressure, gas, and heat when subjected to sudden shock, pressure, or bigh temperature.

A flammable substance is one that will catch on fire and burn rapidly under ordinary conditions; for example, liquids with a flash point below $1000 \mathrm{~F}$ and solids that ignite readily. Note the Uniform Fire Code uses a cut off of $140^{\circ} \mathrm{F}$, which qualifies more liquids as "flammable."

The minimum temperature at which a liquid gives off a vapor in sufficient concentration to ignite. 

formula
$\mathrm{g} / \mathrm{kg}$
The molecular composition of a chemical compound written in scientific symbols. Water is $\mathrm{H}_{2} \mathrm{O}$; hydrochloric acid is $\mathrm{HCl}$.
Grams per kilogram. A measurement used in experimental testing to indicate the dose of a test substance, in grams, given for each kilogram of the test subject's body weight.

hazard warning

hazardous chemical

health hazards

hematopoietic system

hepatotoxin

IARC

ignition temperature
The words, pictures, and symbols, or combination thereof, that appear on a label and indicate the hazards of the substance in the container.

A chemical or mixture of chemicals that can produce adverse physical effects (e.g., fire, explosion) or health effects (e.g., dermatitis, cancer).

Substances for which there is evidence, from at least one scientific study, that acute or chronic health effects may occur in exposed persons. These chemicals include carcinogens, toxic agents, reproductive toxins (mutagens and teratogens), irritants, corrosives, sensitizers, hepatotoxins, nephrotoxins, neurotoxins, agents which act on the hematopoietic system, and agents that damage the lungs, skin, eyes, or muccous membranes.

The blood-forming organs of the body, including bone marrow and the spleen.

A chemical that can cause liver damage (e.g., carbon tetrachloride).

International Agency for Research on Cancer. IARC publishes "Monographs on the Evaluation of the Carcinogenic Risk of Chemicals to Man," one of the publications used to determine the cancer risk of a chemical.

The lowest temperature at which a substance will ignite and continue to burn. The lower the ignition temperature, the more likely the substance is to be a fire hazard. 
ingestion

inhalation

inhibitor

irritant

laboratory-type hood

lacrimation

$\mathrm{LC}_{50}$

$\mathrm{LD}_{50}$

LEL or LFL

local exhaust

$\mathrm{m}^{3}$
Taking a material into the body through the mouth and swallowing it.

Taking a material in the form of a vapor, gas, dust, fume, or mist into the body by breathing it.

A chemical added to a substance to prevent the occurrence of an undesirable chemical reaction.

A substance that may not be corrosive but that can, with direct contact, cause a reversible effect on the skin, eyes, or respiratory system.

A device located in a laboratory, enclosure on five sides with a moveable sash or fixed partial enclosed on the remaining side; constructed and maintained to draw air from the laboratory and to prevent or minimize the escape of air contaminants into the laboratory; and allows chemical manipulations to be conducted in the enclosure without insertion of any portion of the employee's body other than hands and arms.

Abnormal or excessive production of tears as a result of exposure of the eyes to an irritant.

The concentration of a substance in air that will kill half $(50 \%)$ of the exposed test animals. A measure of acute toxicity.

The dose of a substance that will kill half $(50 \%)$ of the treated test animals when given as a single dose. A measure of acute toxicity.

Lower Explosive Limit or Lower Flammable Limit.

A ventilation method for removing contaminated air at the point where the contaminants are generated (e.g., a fume hood).

Cubic meter. A volume measurement in the metric system. One $\mathrm{m}^{3}$ is about 35.3 cubic feet or 1.3 cubic yards. 
mechanical exhaust

$\mathrm{mg} / \mathrm{kg}$

$\mathrm{mg} / \mathrm{m}^{3}$

mist

mucous membranes

mutagen

mutation

narcosis

nephrotoxin

neurotoxin

NFPA
A powered device, e.g., a motor-driven fan, that removes contaminants from a work area or enclosure.

Milligrams per kilogram. A term used in experimental testing to indicate the dose of a test substance, in milligrams, that was given for each kilogram of body weight of the test animal.

Milligrams per cubic meter. A way of expressing the concentration of dusts, gases, aerosols, or mists in the air.

A suspension in air of finely divided particles of liquid.

A protective lining of cells found, for example, in the mouth, throat, nose, and other parts of the respiratory system.

A substance capable of causing damage to genes and chromosomes, particularly those of sperm or egg cells, resulting in mutations.

A genetic alteration that can be inherited, thus affecting future generations.

A state of deep unconsciousness caused by the influence of a drug or other chemical.

A chemical that causes kidney damage (e.g., uranium).

A chemical whose primary toxic effect is on the nervous system (e.g., carbon disulfide).

National Fire Protection Association. This organization provides information on fire protection and prevention. The NFPA 704 "Standard of the Identification of the Fire Hazards of Materials" describes a hazard-warning placarding and labeling system. 
NIOSH

NTP

odor threshold

olfactory

oral

organic peroxide

OSHA

OSP

oxidation
National Institute for Occupational Safety and Health. This agency of the Public Health Service, U.S. Department of Health and Human Services (DHHS), tests and certifies respiratory devices, recommends occupational exposure limits, and assist OSHA by conducting research and investigations.

National Toxicology Program. Publishes "Annual Report on Carcinogens," listing substances either known or anticipated to be carcinogens.

The lowest concentration of a substance's vapor, in air, that a person can detect by smell. Odor thresholds are highly variable, depending on the individual and the nature of the substance.

Refers to the sense of smell.

Refers to the mouth.

A type of oxidizer that is very useful because of its reactive properties, considered by law (OSHA) to be a physical hazard.

Occupational Safety and Health Administration. This government agency develops and enforces occupational safety and health standards for most industry and business in the U.S.

Operational Safety Procedure. A written procedure describing a particularly hazardous operation, explaining the potential hazards, and detailing the methods of mitigating those hazards. The OSP requires EH\&S review.

A reaction in which a substance combines with oxygen to cause chemical change (e.g., fire). In a broader sense, oxidation is a reaction in which electrons are lost and is accompanied by reduction - a reaction in which electrons are gained. 
oxidizer

PEL

$\mathrm{pH}$

physical hazard

polymerization

$\mathrm{ppb}$

ppm

psi

pulmonary
A material that causes the ignition of combustible materials without an external source of ignition. When mixed with combustible materials, an oxidizer increases the rate of burning of these materials when the mixtures are ignited. Oxidizers usually contain their own oxygen, can, therefore, burn in an oxygen-free atmosphere, are usually very unstable or reactive, and pose a serious fire hazard.

Permissible Exposure Limit. The legal maximum amount of a substance allowed by OSHA in workplace air. This limit must not be exceeded.

A measure of how acidic or basic (caustic) a substance is on a scale of 1 (very acidic) to 14 (very basic); pH 7 indicates that the substance is neutral.

A substance that is a combustible liquid, a compressed gas, an organic peroxide, or an oxidizer and is explosive, flammable, pyrophoric, unstable (reactive), or water-reactive.

A chemical reaction in which individual molecules combine to form a single large molecule (a polymer). Hazardous polymerization is an uncontrolled reaction releasing large amounts of energy (heat).

Parts per billion. A measurement used to express very small concentrations of a given substance present in a mixture. Often used as a unit to measure the parts (by volume) of a gas or vapor in a billion parts of air.

Parts per million. A measurement used to express very small concentrations of a given substance present in a mixture. Often used as a unit to measure the parts (by volume) of a gas or vapor in a million parts of air.

Pounds per square inch, a unit of pressure measurement used with compressed gases, etc.

Refers to the lungs. 
pyrophoric

reactivity

reproductive toxins

respiratory protective equipment

route of entry

secondary container

select carcinogen
A chemical that can catch on fire spontaneously in air at or below $130^{\circ} \mathrm{F}$.

A term used to describe the ease with which a chemical can undergo change, usually by reacting with another substance or by breaking down. Highly reactive substance may explode.

Are chemicals that affect the reproductive capabilities including chromosomal damage (mutations) and effects on fetuses (teratogenesis).

Air-cleaning or air-supply respirators that protect against toxic materials in the air.

The means by which a toxic substance enters the body. For example, absorption through the skin, inhalation, ingestion. May also be called mode of entry.

A container into which laboratory personnel transfer material from the vendor supplied container.

means any substance that meets one of the following criteria:

(i) It is regulated by OSHA as a carcinogen; or (ii) It is listed under the category, "known to be carcinogens," in the Annual Report on Carcinogens published by the National Toxicology Program (NTP) (latest edition); or (iii) It is listed under Group 1 ("carcinogenic to humans") by the International Agency for Research on Cancer Monographs (IARC) (latest editions); or (iv) It is listed in either Group 2A or 2B by IARC or under the category, "reasonably anticipated to be carcinogens" by NTP, and causes statistically significant tumor incidence in experimental animals.

A substance that can cause an allergic reaction, which usually appears after repeated exposure. 
solubility in water

solvent

SSP

STEL

suspect carcinogen

synonym

systemic poison

target organ effects

teratogen
Indicates the amount, in \%, of a substance that will dissolve in water. Solubility information is important for determining spill-cleanup and firefighting procedures.

A liquid that dissolves other substances. Some common solvents are water, alcohol, and mineral spirits.

Specific Safety Procedure. A brief, two-page, written safety prodedure that lists the hazardous substances used in an operation (or several similar operations), and outlines the control measures that are to be used. SSPs are used as the basic method for declaring the safety procedures for a specific lab or shop. They do not necessarily require EH\&S review.

Short Term Exposure Limit: The maximum concentration allowed in a continuous, 15-minute exposure. There may be no more than 4 such exposures each day with at least 1 hour between exposures. The daily TWA cannot be exceeded, however.

A substance that might cause cancer in humans but has not yet been proven to do so.

Another name by which a chemical is known. For example, synonyms for methyl alcohol are methanol and wood alcohol.

A substance that has a toxic effect upon several organ systems of the body.

Effects on specific organs of the body caused by exposure to a hazardous chemical.

A chemical or physical agent capable of producing malformation(s) in developing embryo or fetus. 
TLV

TLV-C

TWA

toxic substance

toxicity

UEL

UFL

unstable

vapor

ventilation

vertigo

viscosity
Threshold Limit Value. The airborne concentration of a substance below which no adverse health effects should occur. TLV's, established by the American Conference of Governmental Industrial Hygienists (ACGIH), are voluntary limits expressed in three ways (STEL, TLV-C, TWA).

Threshold Limit Value-Ceiling: The maximum concentration of a toxic substance for which exposure is allowed. This limit is not to be exceeded, even momentarily. The TWA must still be observed.

Time Weighted Average: The exposure limit averaged over a normal 8-hour workday or 40hour workweek.

A substance that causes harmful biological effects after either short-term or long-term exposure.

A relative measure of the adverse biological effects that can result from exposure to a harmful substance.

Upper Explosive Limit.

Upper Flammable Limit.

A chemical is unstable if it tends to decompose or undergo other undesirable chemical changes during normal handling or storage.

The gas given off by a liquid or solid at room temperature.

Circulation and exchange of air and the method by which this is accomplished.

A state of dizziness, and possibly disorientation.

A term used to describe the rate at which a liquid flows or pours. A very viscous liquid, like molasses, flows slowly. 
volatile

water-reactive
A term used for a liquid that evaporates at room temperature. Very volatile liquids, such as gasoline, form vapors (evaporate) quickly and are a breathing hazard.

A chemical that reacts with water to release a flammable or toxic gas. 
Appendix 3

\section{Glossary of Terms}


Appendix 4

The Facility Notebook

and

Specific Safety Procedures (SSPs) 
Appendix 4

The Facility Notebook

and

Specific Safety Procedures (SSPs)

\section{Facility Notebooks}

Lawrence Berkeley Laboratory is responsible for complying with all relevant laws and regulations to maintain a safe and healthy environment for its administrators, scientists, support staff, and visitors. One set of regulations, DOE Order 5480.19, Conduct of Operations, requires the Laboratory to establish a minimum level of uniformity and consistency in its daily operations. The intent of the Order is to assure that operations at DOE facilities are managed, organized, and conducted at an acceptable level of safety. To meet this objective, the Laboratory is implementing the Facility Notebook. The Facility Notebook provides a mechanism for facility administrators to convey facility-specific information on operational policies and procedures, and for facility users to document their adherence to these requirements.

Facility Notebooks will be used in all work environments that have environmental, health and/or safety considerations. Most laboratory and shop work environments have chemical, biological, and/or physical hazards that warrant the need for Facility Notebooks. On the other hand, an administrative office or computer support service area probably does not have significant physical or chemical hazards, and does not require a Facility Notebook. Note that certain occupational illnesses and injuries (e.g., back strains, repetitive stress injuries at computer workstations, etc.) are prevalent throughout any work force but do not, in and of themselves, require a Facility Notebook for the area. However, if significant physical labor is part of the job description for a work force, management might opt to include safety protocols in their Facility Notebook.

The purpose of the Facility Notebook is to provide an organized body of facilityspecific information to assist (1) LBL staff in the safe conduct of their operations, and (2) compliance auditors in their review of facility documentation. It is the logical tool for satisfying the OSHA requirement for hazard determinations and facilityspecific chemical hygiene procedures. 


\section{Implementation of the LBL Chemical Hygiene and Safety Plan (CHSP)}

While the LBL CHSP provides the framework for chemical hygiene procedures, the Laboratory Standard is not fully implemented until facility-specific chemical hygiene information is provided. Facility-specific hazard information and written safety procedures are to be provided by each LBL facility and placed within the Facility Notebook. The Notebook has standardized sections for descriptions of facility organization, communication methods, emergency protocols, training, etc. The Notebook serves to provide the following site-specific information required by the OSHA Laboratory Standard:

- Facility description and chemical hazard identification;

- Operator responsibilities;

- Lab/Shop-Specific Safety Procedures (SSPs) for routine operations using hazardous chemicals;

- Operational Safety Procedures (OSPs) for all extremely hazardous operations (those utilizing toxic gases, high radiation, etc.);

- Chemical hazard information;

- Recordkeeping; and

- Emergency protocols.

One section deals with the Handling of Hazardous Materials, and it is this section that will be discussed in greater detail here because it entails the use of the two-page Specific Safety Procedure, or "SSP."

\section{Specific Safety Procedures (SSPs)}

For most operations and procedures, the SSP will suffice to document safety procedures. For very hazardous operations and procedures, a more detailed Operational Safety Procedure (OSP) is necessary. Both procedures are to be kept with or within the Facility Notebook. The two basic types of written safety procedures are described in greater detail in Section C of this Plan.

Whereas an OSP must be submitted to, reviewed by, and approved by the applicable Safety Review Subcommittee and the Division Director, an SSP is intended as an internal document. It is to be reviewed by the Principal Investigator/Supervisor at least annually and followed by all personnel performing the specific tasks/operations for which it was written. 


\section{The SSP has eight sections for descriptions/discussions:}

I. The Procedure/Operation (e.g., protein extraction, pairiting, electroplating)

II. Hazardous Materials (Identification of the hazardous materials utilized in the operation/procedure)

III. Particularly Hazardous Materials/Agents (Identification of the most hazardous agents in terms of innate toxicity and the potential for exposure)

IV. Hazard Analysis (An analysis describing all potential hazards inherent in the use, storage, and manipulation of these substances)

v. Controls (The intended control measures, i.e., administrative, engineering, and/or personal protective equipment)

VI. Medical Surveillance (Reference to pertinent medical surveillance protocols)

VII. Spill Response/ Decontamination (Provisions for waste handling, spill clean-up, decontamination, etc.)

VIII. Waste Handling (Where waste will be placed, the types of waste, etc.)

\section{How To Fill Out the SSP}

\section{General Information}

At a minimum, a SSP is required for every operation/procedure using chemicals. An OSP is required for extremely hazardous operations. Cne or more SSPs may be necessary for each work area; and, similar procedures may be lumped together on the same SSP. Furthermore, standardized SSPs for common laboratory operations/procedures may be shared among supervisors as long as the details accurately reflect what is practiced.

Project Review Forms, used by the EH\&S Radiation Protection Group, are similar in function and may substitute for the SSP if already in use, or for new start-up projects requiring EH\&S review. EH\&S review is required for all uses of radioactive substances; EH\&S review is not required for chemical use (in the absence of radioactives) unless the written procedure is an OSP. 
How To Fill Out the SSP, cont.

List all personnel authorized to work on a project or in a specified area. Remember, all personnel assigned to any area/project must have received the appropriate training. Also, all personnel named to work with radioactivity or radioactive materials must have submitted a completed LBL Radiation Workers Form RL-6583 to EH\&S.

\section{SSPs, Section-By-Section:}

\section{Procedure/Operation}

Examples of operations and procedures requiring SSPs include:

- Anv hazardous chemical operation not requiring an OSP

- Processes that utilize hazardous chemicals at certain phases of the operation (e.g., some steps in tissue culturing and protein extractions, and the use of high pressure liquid chromatography)

- Use of Class 2 laser systems

- Use of sealed radioactive sources and radionuclides

\section{Hazardous Materials}

From the onset, recognize that the definition of "what" is hazardous varies significantly with the particular EH\&S regulation or policy. For emergency response purposes (such as the Hazardous Materials Management Plan, or "Business Plan") and environmental protection (e.g., waste management, air quality, etc.), almost everything is considered to be potentially hazardous. For occupational safety, the list of hazardous materials is somewhat less lengthy, but complicated by the types of hazards present and the severity of those hazards. The Chemical Hygiene and Safety Plan identifies specific types of hazardous chemicals, such as carcinogens.

Procurement of certain types of hazardous materials is controlled by the Purchasing Department, with assistance from the Industrial Hygiene Group and Radiation 


\section{How To Fill Out the SSP (Section II cont.)}

Assessment Group. Orders for drugs and the drug precursors require special approval prior to purchasing. Similarly, radioactive substances and acutely hazardous materials (e.g., toxic gases, radioactive chemicals) also require preapproval prior to delivery.

An exhaustive chemical inventory is conducted each year for all of LBL. At this time, the inventory is housed on the EMS database, which is available for DOS users through the computer network. Do not attempt to establish a threshold quantity for reporting because agency thresholds are based on the entire LBL site and not a single facility. This task can be conducted on an on-going basis or periodically, but must be done at least annually. The EH\&S Division coordinates the annual LBL inventory of chemicals, radionuclides, and wastes.

For each SSP, identify all chemicals, biohazardous agents, radioactive substances, and human and animal tissues/products that may be used. You may want to attach a printout of all or part of your chemical inventory when preparing the SSP.

For the purposes of this Plan and the SSP, a hazardous chemical is a chemical that can potentially pose a health hazard and/or a physical hazard. The term "health hazard" includes chemicals that are carcinogens, toxic or highly toxic agents, reproductive toxins, irritants, corrosives, sensitizers, liver toxins, kidney toxins, nerve toxins, agents that act on the hematopoietic (blood-forming) system, and agents that damage the lungs, skin, eyes, or mucus membranes.

[Appendices A and B of the Hazard Communication Standard (included in this CHSP as Appendix 1) provide further guidance in defining the scope of health hazards and determining whether or not a chemical is to be considered hazardous. A list of definitions explaining basic hazardous chemical terms is provided in Appendix 3 of this Plan.] 
How To Fill Out the SSP, cont.

\section{Particularly Hazardous Materials/Agents}

\section{Toxins}

The OSHA Laboratory Standard dictates that provisions be in place providing additional employee protection for work with "particularly hazardous substances," described as "select carcinogens, reproductive toxins, and substances with a high degree of acute toxicity." Carcinogens and reproductive toxins are discussed in Sections G.2 and G.3 of this Plan. Consult Appendix 11 for a composite list of OSHA's Select Carcinogens. Consult Appendix 13 for a list of reproductive toxins.

Don't overlook these select carcinogens commonly used in operations and procedures conducted at LBL:

benzene

acrylamide

formaldehyde

methylene chloride chloroform

aniline and benzidine dyes

perchloroethylene

trichloroethylene

Don't overlook these common reproductive toxins:

carbon disulfide

dinitrobenzene

ethylene glycol monoethyl ether

ethylene glycol monomethyl ether ethylene oxide lead and lead compounds mercury and mercury compounds toluene

High acute toxicity substances are defined as those substances such as hydrogen cyanide, hydrogen sulfide, and nitrogen dioxide which "may be fatal or cause damage to target organs as a result of a single exposure or exposures of short duration." High acute toxicity substances include substances that are capable of causing intense irritation that can result in pulmonary edema (fluid and swelling in the lungs), citemical asphyxia, and systemic (body-wide) poisoning. As it happens, mariy substances in this high acute toxicity category are toxic gases, which are discussed in Section G.4. Use of these substances requires the preparation of an OSP. 


\section{How To Fill Out the SSP (Section III cont.)}

\section{Isotopes}

For each isotope, list: (1) the maximum quantity per use, (2) the maximum quantity per shipment container, (3) the possession limit, and (4) the chemical and physical forms. NOTE: If you list any radioactive substances, you must send a copy of the form to the Radiation Assessment Group for their review.

\section{Potential Biohazards}

List all human blood, blood products (e.g. plasma, buffy coat, packed cells), body fluids, tissues, and infectious agents. Use of all except the latter requires that workers receive Blood Biosafety Training (EHS-35) pursuant to the OSHA Bloodborne Pathogen Rule.

\section{Hazard Analysis}

Hazard analysis, or hazard determination, is an evaluation made of the chemicals present in a workplace to determine if they are hazardous. Note that the hazard analysis must be made regardless of the potential for exposure. If there is potential for exposure, other than in minute, trace, or exempt cases (e.g., use of commercially prepared kits within which all the reagents are self-contained), then a hazard determination must be made. The hazard determination may declare that the potential for exposure is low and therefore the risk is low, and that additional control measures are not needed, but the exercise is still conducted and written down in the SSP.

A hazard analysis is not required for hazardous waste (e.g. soil and water samples), or for consumer products that contain hazardous substances if it can be demonstrated that the products are used in the same manner in the workplace as they are in normal consumer use (such that no greater exposure results). However, employees are reminded to use good judgment when using all chemicals, even common household bleach. 


\section{How To Fill Out the SSP (Section IV cont.)}

Describe any special hazards of the project such as the use of volatile or dispersible radioactive materials, the mechanical manipulation of hazardous materials that might increase the likelihood of aerosol generation, the use of pressurized vessels, and intentional chemical reactions that result in heat and/or gas releases, etc.

\section{Controls}

\section{Exposures}

Exposures by inhalation of airborne contaminants (gases, vapors, fumes, dusts, and mists) must not exceed (1) the levels listed in the latest edition of Threshold Limit Values (TLVs) of Airborne Contaminants published by the American Conference of Governmental Industrial Hygienists, (2) OSHA Permissible Exposure Limits (PELs), which are comparable to the TLVs, and (3) the ACGIH and/or OSHA Short Term

Exposure Limits (STELs). These occupational exposure limits are normally published on the manufacturer's Material Safety Data Sheets or available through the Industrial Hygiene Group.

In addition, chemicals can gain entry into the body via the skin and by ingestion. The routes of potential exposure for each type of chemical varies (e.g., some chemicals are more prone to skin absorption than others). Good hygiene protocols summarized in the SSP must address all potential routes of exposure for the chemicals being used.

\section{Selection of Control Measures}

Employee exposures to potential hazards can be controlled through the following basic approaches:

- Administrative controls (e.g., specific work practices, area controls, etc.)

- Engineering controls (e.g., fume hoods)

- Personal protective equipment (e.g., gloves, lab coats, safety glasses) 


\section{How To Fill Out the SSP (Section V cont.)}

All three of these approaches may be used for a single procedure depending upon the circumstances. The control measures appropriate for the particular operation or procedure being conducted depend on a number of factors, including the volatility of the substance, if the substance can be absorbed through the skin (e.g., glycol ethers), and how much of the substance is being used over a time period.

In all cases of potentially harmful exposure, feasible engineering or administrative controls must first be established. In cases where respiratory protective equipment, alone or with other control measures, is required to protect the employee, the protective equipment must be approved by the Industri 1 Hygiene Group for each specific use. [Refer to Sections $G$ and I of his Plan for more information on assessing hazards to determine which controls are best.]

The following factors impact the selection of controls:

- Length of employee exposures (full shift vs. short-term)

- Types of substances being handled or used including the hazardous properties and physical and chemical properties

- The nature of the operation or procedure (i.e., how easily are aerosols or vapors generated).

- Presence of existing controls to reduce exposures

- Number of employees involved

- Quantities of chemical being handled

- Routes of exposure (i.e., how the chemical enters the body)

- Occupational exposure limits

The Laboratory Standard allows flexibility in assessing the hazards presented in using particularly hazardous substances and choosing the control measures that best mitigate those hazards. The only baseline requirement for working with particularly hazardous substances is that consideration be given to the following provisions and that they be utilized when appropriate: 
How To Fill Out the SSP, cont.

(1) establishment of a designated area (e.g., a room, section of a room, or hood);

(2) use of containment devices (e.g., a fume hood);

(3) establishment of contaminated waste removal procedures; and

(4) establishment of decontamination procedures.

\section{(1) Administrative Controls}

"Control areas" are required for the use of radioactive materials; similarly, "designated areas" (or "regulated areas") are required for the use of certain toxins. These area designations are merely a type of administrative control. Area posting, demarcations with floor tape or chains, access control, authorized use limitations, log-in procedures, etc. are all administrative control measures. Specific work practices that are required for safety assurance are also a type of administrative control, and a separate section of the SSP form $(V-4)$ is available to describe these measures.

Requisite to any restricted area is the requirement that personnel working in the area be trained. Appendix 14 provides a check list to help staff ensure that all training topics have been covered. [A separate section of the Facility Notebook is assigned to outline training requirements and to provide documentation of the training.]

Establish designated areas to restrict carcinogen use/storage areas to authorized personnel. Post the areas and be sure that all containers of carcinogens bear a warning label. Do the same thing for reproductive toxins and substances having high levels of acute toxicity (e.g.e toxic gases). Remember thai maintenance and emergency personnel must be advised of the potential problems and hazards before entering these work or storage areas. [The use of designated areas is discussed in Section F.2 of this Plan, "Administrative Controls."] 


\section{How To Fill Out the SSP (Section V, cont.)}

\section{(2) Engineering Controls}

Fume hoods or equivalent containment devices must be considered for handling "select carcinogens," reproductive toxins, and substances which have a high degree of acute toxicity. Circumstances that may require the use of containment devices when using particularly hazardous chemicals include procedures where (1) the chemicals are volatile, (2) aerosol generation is possible, and (3) manipulations or chemical reactions could result in uncontrolled releases. In addition, if employees experience health effects or if the results of exposure monitoring establish that significant employee exposures are possible, a containment device is required.

Generally, the following procedures require a hood:

- Diluting concentrated acids and bases

- Using volatile toxic substances

- Us'ng select carcinogens, reproductive toxins, or highly toxic compounds

- Conducting procedures that generate particulates (e.g., dust) or liquid aerosols (e.g., when vcrtexing) of even moderately toxic chemicals or potentially infectious materials (e.g., human blood)

- Synthesizing or reacting chemicals

- Using odiferous compounds (even if they are relatively safe)

- Using both infectious agents and hazardous chemicals together (requires a properly equipped biological safety cabinet).

Glove boxes are the norm for work with radioactive substances, and biosafety cabinets are often utilized for some radioactive isotopes and potentially infectious agents. List these types of controls. Also, describe any shielding equipment, warnir. 6 devices, detectors, etc. that provide safety controls. 


\section{How To Fill Out the SSP (Section V, cont.)}

[See "Hazard Control Measures" (Section F of this Plan) for a more information on the use of hoods; see "Exposure Monitoring," (Section I) for a discussion on hazard assessment and criteria for monitoring.]

\section{(3) Personal Protective Equipment}

Lab coats are standard for laboratory workers; and, hand protection is usually needed. Other types of personal protective equipment include safety glasses, safety goggles, face shields, boots, booties, Tyveks, lead aprons, hearing protection devices, and rubber aprons.

Consider the substances being used when specifying gloves. Know which chemicals being used are absorbed through the skin and assign gloves appropriate to the task. Often, more than one type of glove is necessary to protect workers for all procedures and/or operations conducted in an area. Consult Appendix 6 for help in selecting the appropriate gloves.

Specify the use of splash guards and rubber aprons for dispensing large amounts of toxins and corrosives.

Respirators are assigned through the Industrial Hygiene Group only, so if you feel that their use is appropriate, please contact the Respirator Program Coordinator at Ext. 4028.

\section{(4) Specific Work Practices}

This type of administrative control is listed separately on the SSP form because good work practices are critical in controlling exposures. Examples of specific work practices that are appropriate for many operations/procedures are listed below:

- Discard used gloves after each use and immediately after any obvious contact with a carcinogen. (Remember that the use of gloves often promotes the spread of contamination because workers fail to change them often enough.) 


\section{How To Fill Out the SSP (Section V, cont.)}

- Transfer toxins in tightly closed containers placed within a durable outer container.

- Dissolve finely divided powdered carcinogens, if possible, into a liquid. This reduces the possibility of generating an aerosol.

- Use mixtures that are as dilute as possible.

- Keep all containers of highly volatile substances and/or toxic substances sealed when not in use.

- Don a lead apron and radiation badge.

- Wash hands between each patient.

\section{Medical Surveillance}

A complete, confidential medical examination is required of all new career employees and those temporary workers hired for periods exceeding three months. Periodic examinations are offered every year at no cost to the employee. All employees are strongly encouraged to participate because it (1) establishes for them a baseline health status (valuable in detecting changes); (2) helps identify "at-risk" workers who may need better protection; (3) identifies those potentially exposed to certain toxic substances who may benefit from medical surveillance; and, (4) provides a good level of free, preventative medicine. Students, pregnant workers, and others who are concerned about their potential exposures are also eligible to contact Health Services for a medical consultation.

"Medical surveillance," which is medical monitoring to protect workers from specific hazards, is required under certain circumstances and for certain substances. Generally, employees identify the hazards in their assigned work areas during the medical evaluation. It is crucial that Health Services be notified of all changes in work assignments, work hazards, and incidents that may have resulted in significant exposures. Consult Section J of this Plan for more information. 


\section{How To Fill Out the SSP (Section VI, cont.)}

All laboratories and shops must participate in a Carcinogen Exposure Assessment Survey. A Carcinogen Exposure Assessment Survey Form (Appendix 12) is to be completed by the Principal Investigator or Laboratory/Shop Supervisor to aid the Industrial Hygiene Group in assessing the potential for on-going and projected exposures to carcinogens. Principal investigators and Lab/Shop Supervisors are to track and submit the names of all personnel using carcinogens.

Refer to Health Services anyone who reports experiencing possible symptoms of exposure, anyone who asks for a medical consultation, and anyone suffering an illness or injury as a result of an accident. Pregnant workers should also make an appointment with Health Services.

\section{Spill Response/Decontamination}

Generally, employees are instructed to contact EH\&S (the Fire Department or the Division Office) when spills occur. However, other remedial actions might also be appropriate (e.g., extinguishing ignition sources, vacating the area, closing the sash, etc.). Specify on the SSP these pocedure-specific actions.

Small chemical spills are often handled at the scene by the regular staff, especially tiny spills/drips of a few mililiters or so. Small spills may be overlooked because workers don't recognize the importance in cleaning them up, or are unfamiliar with the best methods for doing so. Specify on the SSP what workers should do when tiny spills/drips occur (e.g. "Wipe with a papu towel and dispose in the hazwaste can, then rinse with water," "Call the Fire Dept. regardless of quantity," whatever). Another section of the Facility Notebook ("Emergency Procedures") deals with emergencies. General instructions for major spills can be listed in this section, as well as on the SSP.

Specific decontamination procedures may or may not be required. Decontamination is more than spill cleanup; decontamination may be required even when no known spill or release has occurred. For example, decontamination procedures are 


\section{How To Fill Out the SSP (Section VII, cont.)}

warranted for the use of any carcinogen that could leave residual powders, dusts, or films. Decontamination may not be necessary for highly volatile substances (such as methylene chloride) when used in a hood because residual materials are unlikely.

When writing clean-up procedures for carcinogens or other highly toxic substances that are dusts, specify the use of a wet mop or vacuum cleaner equipped with a high efficiency particulate air (HEPA) filter. Do not dry sweep or dry mop toxic dusts.

To facilitate decontamination, specify that work surfaces (stainless steel or plastic trays) be covered with absorbent paper having a moisture-proof lining, or other impervious material. This can be a specified work practice under "Controls."

Protective covering materials are to be decontaminated or disposed of as hazardous waste.

Include protocols for decontaminating contaminated equipment. This is especially applicable for dusts and powders, and potentially biohazardous substances.

\section{Waste Handling}

List the anticipated waste types that will result from the operation/procedure:

- hazardous waste

- radioactive waste

- mixed (hazardous and radioactive) waste

- medical/biohazardous

Describe the waste handling procedures for the particularly hazardous substances (e.g., where they will be transferred, how they will be segregated and stored, and how they will be labeled). List the location of the Satellite Accumulation Area (SAA) that will be used.

Note: Separate sections of the Facility Notebook are provided to address waste handling and waste minimization. 


\section{SAMPLE \\ Specific Safety Procedure (SSP) \\ (p. 1 of 2, SSP \# 4 )}

FACILITY: NONEXISTENT BUILDING DIVISION: LIFE SCIENCE DIVISION

BLDG. NO: 18 ROOM NO.(S): 11,14 PI/ SUPER. INITIALS: TR DATE: $3 / 2 / 92$

PRINCIPAL INVESTIGATOR/SHOP SUPERVISOR: MAX DONALD EXT: 6333

ALTERNATE RESPONSIBLE INDIVIDUAL: SUE SMITH EXT: 6334

I. Procedure/Operation (describe):

Fixation of biologic tissue samples for microscopy.

II. Hazardous Materials (Identify all radioactive substances biohazardous agents, chemicals, human blood products):

- Formaldehyde, $37 \%$ solution (formalin)

- Methanol

- Acetone

III. Particularly Hazardous Materials/Agents Involved:

\begin{tabular}{|l|l}
\hline Materials & Quantity/Duration of Use \\
\hline$\bullet$ Formaldehyde (a "select carcinogen") & $\bullet 500 \mathrm{ml} /$ at one time every 2 wks. \\
\hline & \\
\hline & \\
\hline & \\
\hline
\end{tabular}

IV. Hazard Analysis (describe how the particularly hazardous materials/agents are used and how potential skin or inhalation exposure might occur):

Exposures to formaldehyde will be kept as low as possible because all chemical dispensing, tissue fixing, and handling of newly fixed tissues will be conducted in the fume hood. Skin absorption will be prevented by wearing suitable gloves. 
V. Controls:

\section{SAMPLE SSP continued}

(p. 2 of 2 SSP \# 4 )

(1) Administrative (e.g. controlled areas for radiation, designated or regulated areas for carcinogens, storage requirements, worker rotation, specific training, etc.): All users must have received training in the generation of hazardous wastes, the generation of medical waste, Chemical Hygiene \& Safety Training, and bench training. Carcinogen use is limited to the hood and the hood is posted to this effect. Store acetone \& methanol. in flam. cabinet

(2) Engineering (e.g. hoods, biosafety cabinets, etc.): Formalin solutions are to be used and dispensed in the laboratory fume hood to minimize worker exposure. Formalin may not be used in the biosafety cabinet.

(3) Personal Protective Equipment (e.g. gloves, aprons, lab coats, respirators, etc.): - protective eyewear (e.g., safety glasses with side shields, goggles, or faceshield)

- lab coat, or lab coat and apron when dispensing large quantities ( $>1$ liter)

- neoprene or nitrile gloves ("Best" gloves for delicate work with tissue slides)

(4) Specific Work Practices (e.g. type of disinfectant, use of centrifuge cups, leak checks, logging, etc.)

- Decant waste in the hood prior to transfer to the WAA.

- Do not remove newly fixed tissues from the hood for at least two hours after fixing them. - Store flammables in flammable storage cabinets.

- Dispose of soiled gloves in the sealed dry waste container for hazardous waste to prevent "off-gassing." - Keep containers tightly closed.

VI. Medical Surveillance (If appropriate):

Fill out a Carcinogen Exposure Assessment Survey form and return it to the Industrial Hygiene Group. This will identify all staff members who work with carcinogens. Also, refer anyone to Health Services who reports experiencing possible symptoms of exposure, including dermal sensitization.

VII. Spill response/decontamination: In the event of a spill, shut off nearby ignition sources. If the spill is small, wear suitable personal protective equipment, contain the spill with absorbent clean-up material, and dispose of waste in the hazardous waste can. For large spills, contact EH\&S.

VIII. Waste Handling (define types generated, where deposited, etc.): Follow protocols in PUB-3092 for generating hazardous waste. Label the formalin can with "Waste formaldehyde - Flammable, Carcinogen." 


\section{LAB/SHOP \\ SPECIFIC SAFETY PROCEDURE (SSP) \\ (p. 1 of 2, SSP\#_}

FACILITY:

BLDG. NO: ROOM NO.(S):

DATE:

PRINCIPAL INVESTIGATOR/SHOP SUPERVISOR: EXT:

ALTERNATE RESPONSIBLE INDIVIDUAL: EXT:

I. Procedure/Operation (describe): I. Hazardous Materials (Identify all radioactive substances, biohazardous agents, chemicals, human
blood products):

III. Particularly Hazardous Materials/Agents Involved:

\begin{tabular}{|c|c|}
\hline Materials & Quantity/Duration of Use \\
\hline & \\
\hline & \\
\hline & \\
\hline & \\
\hline
\end{tabular}

IV. Hazard Analysis (describe how the particularly hazardous materials/agents are used and how potential skin or inhalation exposure might occur): 


\section{LAB/SHOP \\ SPECIFIC SAFETY PROCEDURE (SSP) \\ (p. 2 of 2 SSP \#__ )}

V. Controls:

(1) Administrative (e.g. controlled areas for radiation, designated or regulated areas for carcinogens, storage requirements, worker rotation, specific training, etc):

(2) Engineering (e.g. hoods, biosafety cabinets, etc):

(3) Personal Protective Equipment (e.g. gloves, aprons, lab coats, respirators, etc.):

(4) Specific Work Practices (e.g. type of disinfectant, use of centrifuge cups, leak checks, logging, etc.)

VI. Medical Surveillance (If appropriate):

VII. Spill response/decontamination:

VIII. Waste Handling (define types generated, where deposited, etc.): 
Appendix 5

University of California Purchase Requisition 


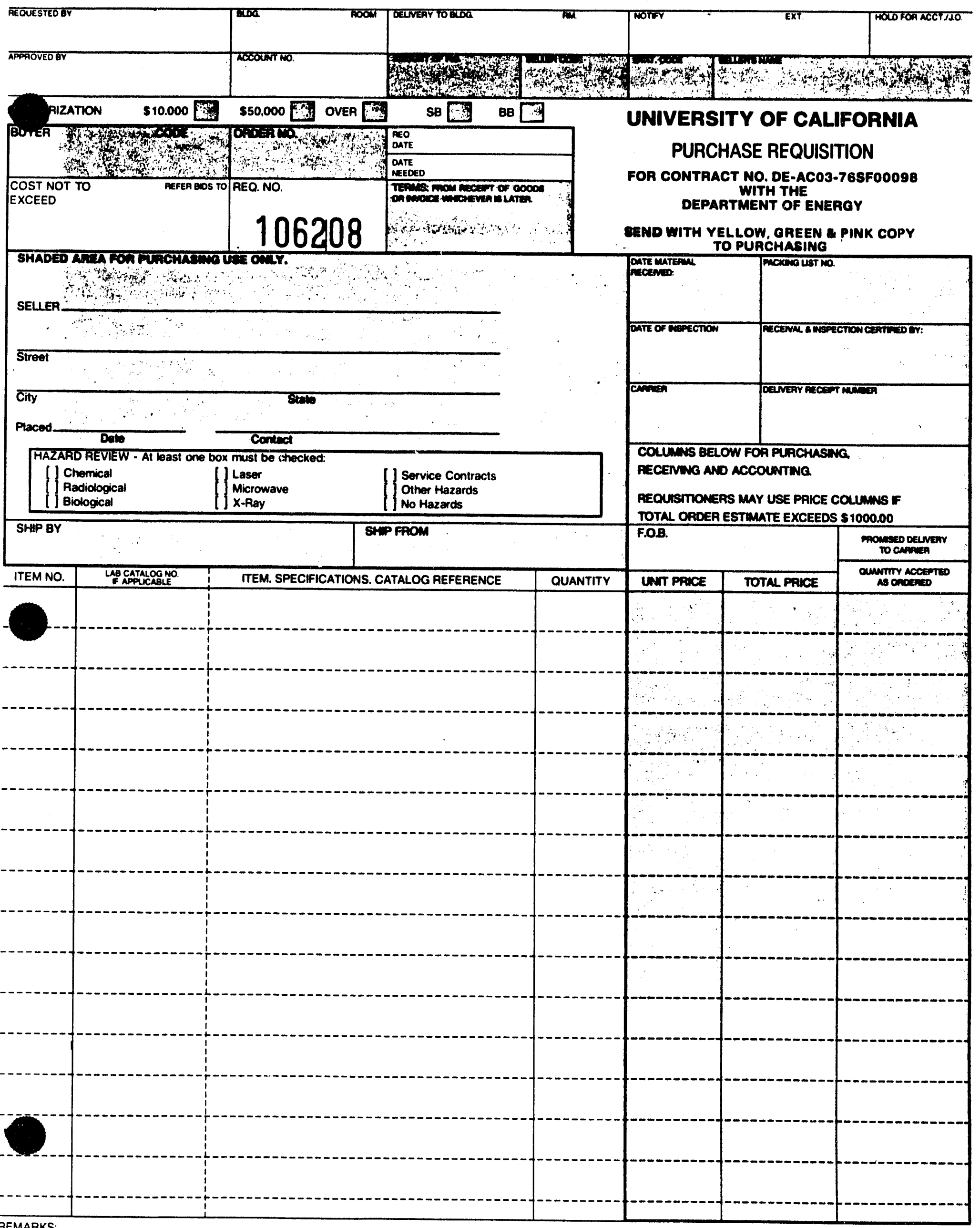




\section{Appendix 6}

\section{Glove Selection Guides}




\section{Appendix 6}

\section{Glove Selection Guides}

\section{The Right Glove for the Job}

The first step in choosing the right glove is determining your primary concern. Do you need protection from hazardous chemicals? Is dexterity crucial to your work? Is product protection of utmost importance?

Different glove materials offer different kinds of protection. Neoprene provides chemical/oil resistance while nitrile adds abrasion resistance to that protection. Vinyl gives you economical flexibility as a natural rubber alternative. Natural rubber latex offers inherent elasticity and resiliency, plus the dexterity needed in food processing or pharmaceutical manufacturing.

Because a materials suitability may be affected by either degradation or permeation, both factors must also be considered when selecting appropriate gloves. Degradation is the reduction in one or more of physical properties of a material due to chemical contact. Exposed gloves may swell, get harder or softer, stiffen or weaken or become brittle. Permeation is the passage of a chemical material even if the material is not susceptible to chemical attack. Permeation can occur even if there is no visible damage to the gloves being worn. Since there is usually no indication that a glove has been permeated, the person wearing gloves to handle hazardous chemicals can get a false sense of security.

The selection of appropriate gloves and other pieces of Chemical Protective Clothing (CPC) is generally done after consulting one or more chemical degradation guides. This app ndix contains three such guides:

Guide \#1 the physical properties of several materials.

Guide \#2 the degradation properties of several materials.

Guide \#3 the permeation properties of several materials. 
The following listing will help you understand the various glove related terms used in the guides:

Natural Rubber: A material (also called latex) that is inherently elastic and resilient, plus resists acids, alkalies, salts and ketones. Natural rubber gloves are suited for food processing, electronics assembly and laboratory chemical handling.

Neoprene: A synthetic rubber developed as an oil-resistant substitute for natural rubber. It also resists a broad range of chemicals. Neoprene gloves are used in petrochemical, degreasing and refining applications, and when handling acids, caustics, alcohols and solvents.

Nitrile: A synthetic rubber with superior puncture and abrasion resistance in addition to chemical protection. Nitrile gloves are suited for stripping and degreasing, as well as acid etching and chemical washing.

PVC: Also known as polyvinyl chloride or vinyl, PVC is a plastic material that resists acids and alcohols, but not petroleum products. Vinyl gloves are used for intricate assembly work, food processing, laboratory, research, and pharmaceutical menu.

Viton: A specialy fluoroelastomer which has excellent resistance to oils, fuels, lubricants, most mineral acids, hydraulic fluids and aliphatic and aeromatic hydrocarbons.

CPE: This chlorinated polyethylene has increased resistance to oil, ozone, heat and chemicals. It also provides low permeability to gases.

Supported: A supported glove has a fabric liner that is coated with a polymer. The liner is generally knit, and can be palm-coated or fully coated. Supported gloves deliver more durable hand protection.

Unsupported: Refers to gloves produced by dipping a glove form directly into a compound, yielding a glove that is $100 \%$ compound. Unsupported gloves offer better tactile sensitivity and dexterity. 
This Chemical Resistance Chart is offered as a general guide to chemical attack. The ratings are based upon data from laboratory tests and published literature from the various manufacturers of rubber and polymers.

To select the proper glove for your chemical handling application, you must test to determine which liquid-proof type is most suitable. First determine the chemical and physical conditions present, then choose the glove with the highest performance ratings for those conditions.

You are now ready to test your selection under actual use conditions. You must determine suitability based on your own performance requirements.

Special Note: All chemicals are potentially hazardous, and should therefore be handled with care. Many chemicals are highly toxic, or are considered known or suspected human carcinogens including some of those listed in the chart. The chart indicates only comparative resistance to the degrading effect of these chemicals. Permeation resistance is not considered in these ratings. Permeation can occur even if there is no visible damage to the glove.

As a general rule, gloves made by the cement dip process exhibit a greater resistance to permeation than gloves made by the latex dip process. Therefore, it is recommended that you select a cement dip glove for evaluation in applications involving the handling of toxic or carcinogenic (known or suspected) chemicals. All Viton, butyl, and natural rubber gloves made by North Hand Protection are manufactured by the cement dip process.

The information contained in this chart is advisory only. North assumes no responsibility for the suitability of particular user glove selections.

Key to Chemical Chart:

E. Excellent - Fluid has no effect

G. Good - Fluid has minor effect

F. Fair - Fluid has moderate effect

P. Poor - Fluid has severe effect, ranging from moderate to complete destruction

Blank - No data or insufficient evidence

Physical Performance Chart

PHYSICAL NATURAL

CHARACTERISTICS NITRILE BUTYL RUBBER PVC VITON

$\begin{array}{llllll}\text { Abrasion Resistance } & \text { E } & \text { G } & \text { G } & \text { G } & \text { G } \\ \text { Cut Resistance } & \text { E } & \text { G } & \text { E } & \text { P } & \text { G } \\ \begin{array}{l}\text { Puncture (Snag) } \\ \quad \text { Resistance }\end{array} & \text { E } & \text { G } & \text { E } & \text { G } & \text { G } \\ \text { Flexibility } & \text { E } & \text { G } & \text { E } & \text { G } & \text { G } \\ \text { Heat Resistance } & \text { G } & \text { G } & \text { F } & \text { P } & \text { G } \\ \text { ()rone Resistance } & \text { F } & \text { G } & \text { P } & \text { E } & \text { E } \\ \text { Tensile Strength } & \text { E } & \text { G } & \text { E } & \text { F } & \text { G } \\ \text { Low Gas Permeability } & \text { P } & \text { E } & \text { F } & \text { P } & \text { E }\end{array}$

Note Products in these categories vary in capabilities. Laboraton' tests are necessary for specific recommendations.

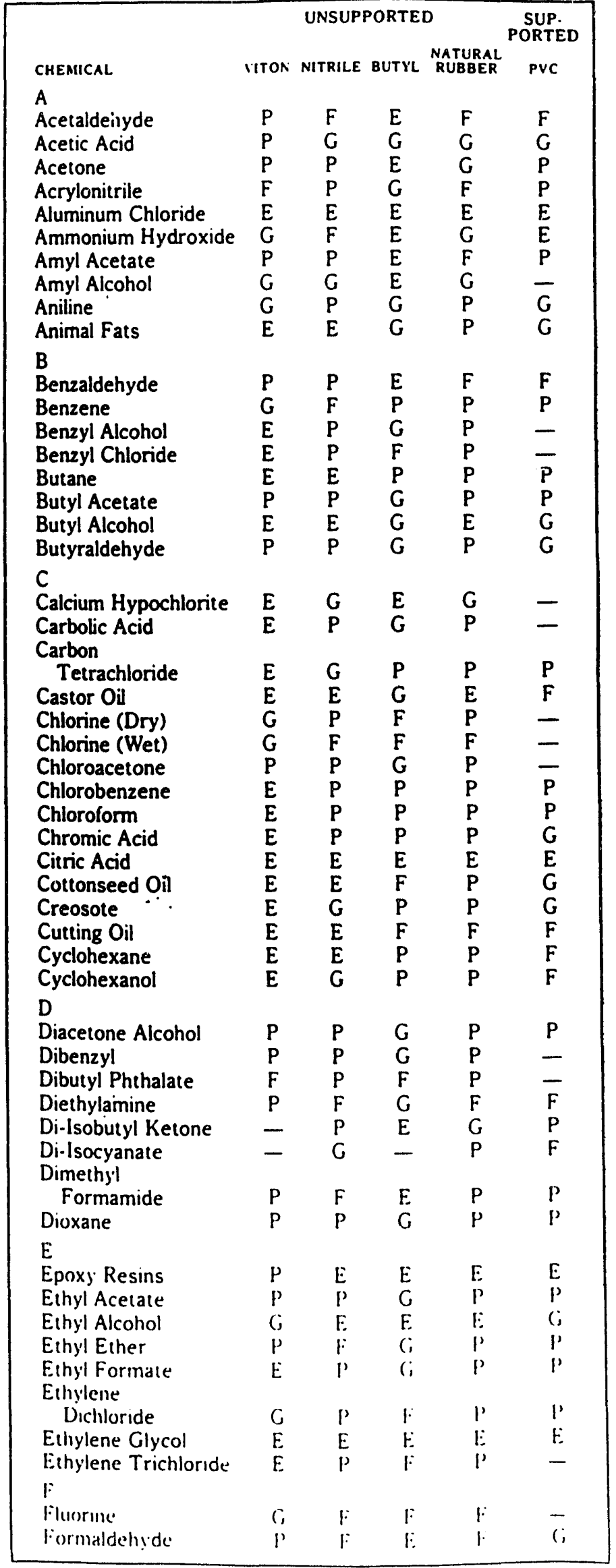




\begin{tabular}{|c|c|c|c|c|c|}
\hline \multirow[b]{2}{*}{ CHFMICH. } & \multirow[b]{2}{*}{ VITON } & \multicolumn{3}{|c|}{ LNSLPPORTED } & \multirow{2}{*}{$\begin{array}{l}\text { SLPP. } \\
\text { PORTED } \\
\text { PNC }\end{array}$} \\
\hline & & NITRII.F. & BITIL & $\begin{array}{l}\text { PATIRRA. } \\
\text { RI'BBE:R }\end{array}$ & \\
\hline$F$ & & & & & \\
\hline $\begin{array}{l}\text { Formic Acid } \\
\text { Freon } 11-12-21.22\end{array}$ & $\mathrm{~F}$ & $\mathrm{~F}$ & $E$ & E & $E$ \\
\hline Freon 11 & G & G & $P$ & $P$ & - \\
\hline Freon 12 & E & G & G & G & - \\
\hline Freon 21 & $P$ & $\mathrm{P}$ & $\mathrm{P}$ & $P$ & - \\
\hline Freon 22 & $\mathbf{P}$ & $P$ & $E$ & $E$ & - \\
\hline Furfural & $P$ & $P$ & G & $\mathbf{P}$ & $\mathrm{P}$ \\
\hline $\begin{array}{l}\text { G } \\
\text { Gasoline } \\
\text { Glicerin }\end{array}$ & $\begin{array}{l}E \\
E\end{array}$ & $\begin{array}{l}E \\
E\end{array}$ & $\begin{array}{l}P \\
E\end{array}$ & $\begin{array}{l}P \\
E\end{array}$ & $\begin{array}{l}P \\
F\end{array}$ \\
\hline $\mathrm{H}$ & & & & & \\
\hline Hexane & $E$ & $E$ & $P$ & $\mathrm{P}$ & $\mathrm{F}$ \\
\hline $\begin{array}{l}\text { Petroleum Base } \\
\text { Hydraulic Fluid- }\end{array}$ & E & $E$ & $\mathrm{P}$ & $\mathrm{P}$ & G \\
\hline Ester Base & $\mathrm{F}$ & $\mathbf{P}$ & G & $\mathrm{P}$ & $\mathbf{P}$ \\
\hline $\begin{array}{l}\text { Hydrobromic Acid } \\
\text { Hydrochloric Acid }\end{array}$ & $E$ & $P$ & $\mathrm{E}$ & $E$ & - \\
\hline $37 \%$ & E & G & E & G & $G$ \\
\hline Hydrofluoric Acid & G & $\mathrm{F}$ & G & $\mathrm{F}$ & $\mathrm{F}$ \\
\hline Hydrogen Peroxide & $E$ & $\mathrm{~F}$ & G & $F$ & $\mathrm{~F}$ \\
\hline Hydroquinone & G & $F$ & $\mathrm{P}$ & G & $F$ \\
\hline 1 & & & & & \\
\hline Isobutyl Alcohol & $E$ & G & $E$ & $E$ & G \\
\hline Iso-Octane & $E$ & $E$ & P & $\mathrm{P}$ & $P$ \\
\hline Isopropyl Alcohol & $E$ & G & $E$ & E & G \\
\hline K & & & & & \\
\hline nerosene & $\mathbf{E}$ & E & $P$ & $P$ & $r$ \\
\hline L a reic said & & & $r$ & $r$ & $c$ \\
\hline Lard & E & E & $\stackrel{L}{F}$ & P & - \\
\hline Linseed Oil & $E$ & $E$ & G & $\mathrm{P}$ & $\mathbf{F}$ \\
\hline $\begin{array}{l}\text { Lubricating Oils } \\
\text { (Petroleum) }\end{array}$ & $E$ & $\mathrm{E}$ & $\mathbf{P}$ & $P$ & - \\
\hline M & & & & & \\
\hline Maleic Acid & $E$ & $P$ & $\mathbf{P}$ & $P$ & $G$ \\
\hline Methyl Acetate & $P$ & $P$ & G & $P$ & - \\
\hline Methyl Alcohol & P & $E$ & $E$ & $E$ & $F$ \\
\hline Methyl Bromide & $E$ & G & $\mathrm{F}$ & $\mathrm{F}$ & $P$ \\
\hline Methịl Cellosolve & $P$ & $F$ & $\mathrm{G}$ & $P$ & - \\
\hline Methilene Chloride & $G$ & $\mathrm{P}$ & $F$ & $P$ & $P$ \\
\hline Methil Ethyl & & & & & \\
\hline Ketone (M.E.K.) & $P$ & $\mathrm{P}$ & $E$ & G & $P$ \\
\hline Methỵl Formate & - & $P$ & G & $P$ & - \\
\hline Methil Isobutyl & & & & & \\
\hline Ketone & $\mathrm{P}$ & $\mathrm{P}$ & G & $F$ & $F$ \\
\hline Methỵlamine & - & $\mathrm{F}$ & G & $F$ & $\mathrm{~F}$ \\
\hline Methỵl & & & & & \\
\hline Meihacrylate & $\mathrm{P}$ & $P$ & $\mathrm{~F}$ & $\mathrm{P}$ & $\mathrm{F}$ \\
\hline Mineral Oil & $E$ & $E$ & $\mathrm{P}$ & $P$ & $F$ \\
\hline Monoethanolamine & $P$ & $P$ & G & G & C; \\
\hline Morpholme & - & $\mathrm{P}$ & G & F & (; \\
\hline Muriatic Acid & - & G & $E$ & (j) & (; \\
\hline$\therefore$ & & & & & \\
\hline Naphtha & E. & $E$ & $P$ & $P$ & $P$ \\
\hline Nitric teid - & & & & & \\
\hline Concentrated & (; & !' & F & P' & $F$ \\
\hline Nitre tind - & & & & & \\
\hline Dilute? & G & $F$ & F & 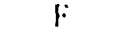 & (j) \\
\hline
\end{tabular}

\begin{tabular}{|c|c|c|c|c|c|}
\hline \multirow[b]{2}{*}{ CHEMICAL. } & \multirow[b]{2}{*}{ vitos } & \multicolumn{3}{|c|}{ UNSUPPORTED } & \multirow{2}{*}{$\begin{array}{c}\text { SI'p. } \\
\text { portil) } \\
m\end{array}$} \\
\hline & & NITRILE. & BITTL. & $\begin{array}{l}\text { RITIRAN. } \\
\text { RTBEFR }\end{array}$ & \\
\hline \multirow{2}{*}{\multicolumn{6}{|c|}{$\begin{array}{l}N \\
\text { Nitric Acid }\end{array}$}} \\
\hline Nitric Acid - & & & & & \\
\hline $\begin{array}{l}\text { Red Funing } \\
\text { Nitrobenzene }\end{array}$ & $\mathrm{F}$ & $\begin{array}{l}P \\
F\end{array}$ & $\mathrm{P}$ & $p$ & $\begin{array}{l}1 \\
1\end{array}$ \\
\hline Nitromethane & $\mathrm{P}$ & $P$ & G & G & 1 \\
\hline \multicolumn{6}{|l|}{0} \\
\hline Octyl Alcohol & $E$ & G & G & G & r: \\
\hline Oleic Acid & $G$ & $F$ & $\mathrm{P}$ & $P$ & f: \\
\hline Olive Oil & $E$ & $E$ & G & $\mathrm{P}$ & $F$ \\
\hline Oxalic Acid & $E$ & G & $E$ & G & ； \\
\hline \multicolumn{6}{|l|}{$P$} \\
\hline PCBs & $\mathrm{E}$ & $F$ & $\mathrm{P}$ & $P$ & $P$ \\
\hline Paint Remover & G & G & $\mathrm{P}$ & $\mathrm{F}$ & 1 \\
\hline Pentane & $E$ & $E$ & $\mathrm{P}$ & $\mathrm{P}$ & $p$ \\
\hline Perchloric Acid & $E$ & $\mathbf{P}$ & G & $\mathrm{P}$ & $P$ \\
\hline Perchloroethylene & $E$ & $F$ & $\mathrm{P}$ & $P$ & $P$ \\
\hline Phenol & $E$ & $\mathrm{P}$ & $G$ & $P$ & G \\
\hline Phosphoric Acid & $\mathrm{E}$ & G & $\mathrm{E}$ & G & $P$ \\
\hline Pickling Solution & $\bar{F}$ & $\mathrm{P}$ & $\bar{F}$ & $\mathrm{P}$ & G \\
\hline Picric Ácid & $E$ & G & $G$ & G & $G$ \\
\hline Pine Oil & $E$ & G & $\mathrm{P}$ & $\mathbf{P}$ & $F$ \\
\hline \multicolumn{6}{|l|}{ Plating Solutions - } \\
\hline \multicolumn{6}{|l|}{ Potassium Hydroxide } \\
\hline$(50 \%)$ & $F$ & G & $E$ & G & $E$ \\
\hline Printing Ink & - & $E$ & - & G & $\mathrm{F}$ \\
\hline Propane & $E$ & $\mathbf{E}$ & $P$ & $\mathrm{P}$ & $\mathrm{F}$ \\
\hline Propyl Acetate & $\mathrm{P}$ & $\mathbf{P}$ & G & $\mathrm{P}$ & $\mathrm{F}$ \\
\hline Propyl Alcohol & $E$ & $\mathrm{E}$ & $E$ & $E$ & G \\
\hline Propylene & $E$ & $\mathbf{P}$ & $\mathrm{P}$ & $P$ & - \\
\hline \multicolumn{6}{|l|}{$\mathrm{S}$} \\
\hline Sky'drol 500 & $F$ & $\mathrm{P}$ & G & $\mathrm{P}$ & $\mathrm{P}$ \\
\hline \multicolumn{6}{|l|}{ Sodium Hydroxide } \\
\hline$(50 \%)$ & $G$ & G & $E$ & $E$ & $\mathrm{E}$ \\
\hline Sodium Hypochlorite & $E$ & G & $\bar{E}$ & $\mathrm{G}$ & - \\
\hline Soybean Oil & $E$ & $\mathrm{E}$ & $\mathrm{F}$ & $P$ & - \\
\hline Stearic Acid & - & G & G & G & G \\
\hline Stoddard Solvent . & $E$ & $\mathbf{E}$ & $P$ & $P$ & $F$ \\
\hline Styrene & G & $P$ & $P$ & $\mathrm{P}$ & $P$ \\
\hline Sulfuric Acid (Diluted) & $E$ & G & $G$ & G & $G$ \\
\hline \multicolumn{6}{|l|}{ Sulfuric Acid } \\
\hline (Concentrated) & $E$ & $P$ & G & $P$ & $F$ \\
\hline \multicolumn{6}{|l|}{$\mathrm{T}$} \\
\hline Tannic Acid & $E$ & $E$ & $E$ & $E$ & $E$ \\
\hline Tetrahịdrofuran & $\mathrm{P}$ & $P$ & G & $P$ & $p$ \\
\hline Toluene & $\mathrm{E}$ & $\mathrm{F}$ & $\mathrm{P}$ & $P$ & $r$ \\
\hline \multicolumn{6}{|l|}{ Toluene } \\
\hline Di-Isocyanate & $\varphi$ & $\mathrm{P}$ & $G$ & 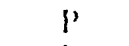 & $P$ \\
\hline Trichlorethylene & $E$ & $F$ & $\mu$ & $\mu$ & 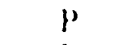 \\
\hline Tricres!l Phosphate & $G$ & $\mathrm{P}$ & $\mathrm{E}$ & $p$ & $p$ \\
\hline Triethanol Anine & $p$ & $\mathrm{~F}$ & $\ddot{G}$ & G & i \\
\hline Trinitrolsoluene & G & $p$ & $p$ & $l^{\prime}$ & $r$ \\
\hline Tung (Bil & $\mathrm{E}$ & $E$ & $F$ & $p$ & $F$ \\
\hline Turbine ()il & $\mathrm{E}$ & ； & r & $l^{\prime}$ & $p$ \\
\hline Turpentune & $\mathrm{F}$ & $E$ & $P$ & $P$ & F \\
\hline \multicolumn{6}{|l|}{ 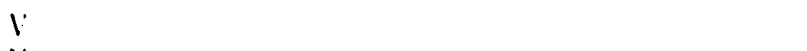 } \\
\hline Vegertable $O_{11}$ & $E$ & $E$ & $\mathrm{E}$ & $l^{\prime}$ & $F$ \\
\hline ling̣l Chlonde & ； & - & - & - & - \\
\hline \multicolumn{6}{|l|}{$x$} \\
\hline Xivene. & $E$ & F & $r^{\prime}$ & $r^{\prime}$ & $l^{\prime}$ \\
\hline
\end{tabular}




\begin{tabular}{|c|c|c|c|c|c|c|c|c|c|c|}
\hline \multirow[b]{2}{*}{ CHEMICAL } & \multicolumn{2}{|c|}{$20.42 \times C$} & \multicolumn{2}{|c|}{ XSPCNIIRULE } & \multicolumn{2}{|c|}{ Yर \&NITILE } & \multicolumn{2}{|c|}{ MATURAL RUBBER } & \multicolumn{2}{|c|}{ SESMEOPRENE M M } \\
\hline & \begin{tabular}{|c|} 
Breakthrough \\
Time
\end{tabular} & $\begin{array}{c}\text { Steacy State } \\
\text { Rate }\end{array}$ & \begin{tabular}{|c|} 
Breakthrough \\
Time
\end{tabular} & $\begin{array}{c}\text { Steady State } \\
\text { Rate }\end{array}$ & \begin{tabular}{|c|} 
Breakthrough \\
Time
\end{tabular} & \begin{tabular}{|c|}
$\begin{array}{c}\text { Steadof Strate } \\
\text { Rale }\end{array}$ \\
\end{tabular} & \begin{tabular}{|c|}
$\begin{array}{c}\text { Breakthrough } \\
\text { Time }\end{array}$ \\
\end{tabular} & $\begin{array}{c}\text { Sieady State } \\
\text { Rale }\end{array}$ & \begin{tabular}{|c|} 
Breakthrough \\
Time
\end{tabular} & \begin{tabular}{|c|} 
Steacos state \\
Rate
\end{tabular} \\
\hline $\begin{array}{l}\text { Acetic Acid (16N) } \\
\text { Acetone } \\
\text { Ammonium Hydroxide (22Be) } \\
\text { Amyl Acetate } \\
\text { Aniline } \\
\text { Benzene } \\
\text { Butyl Acetate } \\
\text { N-Butyl Alcohol (N-Butanol) } \\
\text { Chlorothene } \\
\text { Chromic Acid (10N) }\end{array}$ & $\begin{array}{c}4 \mathrm{hrs} . \\
18 \mathrm{~min} . \\
3 / 4 \mathrm{hr} . \\
1 / 2 \mathrm{hr} . \\
4 \mathrm{hrs} . \\
1 / 2 \mathrm{hr} . \\
20 \mathrm{~min} . \\
2 \mathrm{hrs} . \\
1 / 2 \mathrm{hr} . \\
6 \mathrm{hrs} .\end{array}$ & \begin{tabular}{|c|}
2 \\
90 \\
- \\
8 \\
1.4 \\
40 \\
12 \\
2.5 \\
20 \\
0
\end{tabular} & $\begin{array}{c}6 \mathrm{hrs} . \\
1 / 4 \mathrm{hr} . \\
3 \mathrm{hrs} . \\
50 \mathrm{~min} . \\
6 \mathrm{hrs} . \\
7 / 4 \mathrm{hr} . \\
2 / 3 \mathrm{hr} . \\
6 \mathrm{hrs} . \\
50 \mathrm{~min} . \\
6 \mathrm{hrs} . \\
\end{array}$ & $\begin{array}{c}0 \\
52 \\
- \\
7 \\
0 \\
30 \\
10 \\
0 \\
16 \\
0\end{array}$ & $\begin{array}{l}6 \mathrm{hrs} . \\
1 / 3 \mathrm{hr} . \\
6 \mathrm{hrs} \\
2 / 3 \mathrm{hr} . \\
21 / 2 \mathrm{hrs} . \\
1 / 3 \mathrm{hr} . \\
11 / 3 \mathrm{hrs} . \\
6 \mathrm{hrs} . \\
2 \mathrm{hrs} . \\
6 \mathrm{hrs} .\end{array}$ & $\begin{array}{c}0 \\
80 \\
0 \\
5 \\
5 \\
150 \\
15 \\
0 \\
100 \\
0\end{array}$ & $\begin{array}{l}2 \mathrm{hrs} . \\
6 \mathrm{~min} . \\
2 \mathrm{hrs} . \\
1 / 5 \mathrm{hr} . \\
1 \mathrm{hr} . \\
11 \mathrm{~min} . \\
8 \mathrm{~min} . \\
2 \mathrm{hrs} . \\
8 \mathrm{~min} . \\
11 / 6 \mathrm{hrs} .\end{array}$ & $\begin{array}{c}- \\
10 \\
- \\
10 \\
1 \\
66 \\
36 \\
2 \\
150 \\
-\end{array}$ & $\begin{array}{c}6 \mathrm{hrs} . \\
6 \mathrm{~min} . \\
6 \mathrm{hrs} . \\
1 / 4 \mathrm{hr} . \\
1 / 2 \mathrm{hr} . \\
1 / 4 \mathrm{hr} . \\
1 / 4 \mathrm{hr} . \\
6 \mathrm{hrs} . \\
1 / 5 \mathrm{hr} . \\
11 / 4 \mathrm{hrs} .\end{array}$ & $\begin{array}{c}- \\
12 \\
0 \\
11 \\
2 \\
93 \\
12 \\
0 \\
130 \\
-\end{array}$ \\
\hline $\begin{array}{l}\text { Cyclohexane } \\
\text { Oyclohexanol } \\
\text { Di-lsobutyl Ketone } \\
\text { Dimethyl Formamide } \\
\text { Dimethyl Sulfoxide } \\
\text { Ethyl Acetate } \\
\text { Ethyl Alcohol (Ethanol, Grain Alcohol) } \\
\text { Ethylene Glycol } \\
\text { Ethyl Ether } \\
\text { Furfural }\end{array}$ & $\begin{array}{c}2 / 3 \mathrm{hr} . \\
6 \mathrm{hrs} . \\
1 \mathrm{hr} . \\
1 \mathrm{hr} . \\
50 \mathrm{~min} . \\
20 \mathrm{~min} . \\
21 / 2 \mathrm{hrs} . \\
6 \mathrm{hrs} . \\
20 \mathrm{~min} . \\
1 \mathrm{hr} .10 \mathrm{~min} .\end{array}$ & $\begin{array}{c}36 \\
0 \\
1.4 \\
23 \\
1 \\
13 \\
1 \\
0 \\
350 \\
18\end{array}$ & $\begin{array}{c}3 \mathrm{hrs} . \\
6 \mathrm{hrs} . \\
11 / 4 \mathrm{hrs} . \\
11 / 2 \mathrm{hrs} . \\
11 / 3 \mathrm{hrs} . \\
1 / 2 \mathrm{hr} . \\
6 \mathrm{hrs} . \\
6 \mathrm{hrs} . \\
25 \mathrm{~min} . \\
2 / 3 \mathrm{hr} .\end{array}$ & $\begin{array}{c}2 \\
0 \\
0.5 \\
22 \\
0.8 \\
8 \\
0 \\
0 \\
310 \\
24\end{array}$ & $\begin{array}{l}6 \mathrm{hrs} . \\
6 \mathrm{hrs} . \\
43 / 4 \mathrm{hrs} . \\
1 \mathrm{hr} . \\
41 / 3 \mathrm{hrs} . \\
1 / 2 \mathrm{hr} . \\
6 \mathrm{hrs} . \\
6 \mathrm{hrs} . \\
21 / 2 \mathrm{hrs} . \\
55 \mathrm{~min} .\end{array}$ & $\begin{array}{l}0 \\
0 \\
5 \\
20 \\
0.9 \\
11 \\
0 \\
0 \\
14 \\
26\end{array}$ & $\begin{array}{l}6 \mathrm{~min} . \\
25 \mathrm{~min} . \\
1 / 4 \mathrm{hr} . \\
1 \mathrm{hr} . \\
11 / 3 \mathrm{hrs} . \\
11 \mathrm{~min} . \\
11 / 2 \mathrm{hrs} . \\
6 \mathrm{hrs} . \\
10 \mathrm{~min} . \\
1 / 4 \mathrm{hr} .\end{array}$ & $\begin{array}{c}340 \\
12 \\
97 \\
120 \\
120 \\
9 \\
1 \\
0 \\
260 \\
5\end{array}$ & $\begin{array}{l}6 \mathrm{~min} . \\
3 \mathrm{hrs} . \\
1 / 4 \mathrm{hr} . \\
8 \mathrm{~min} . \\
6 \mathrm{hrs} . \\
1 / 5 \mathrm{hr} . \\
2 \mathrm{hrs} . \\
6 \mathrm{hrs} . \\
1 / 5 \mathrm{hr} . \\
1 / 2 \mathrm{hr} .\end{array}$ & $\begin{array}{c}180 \\
10 \\
75 \\
16 \\
0 \\
8 \\
0.5 \\
0 \\
205 \\
3\end{array}$ \\
\hline $\begin{array}{l}\text { N-Hexane } \\
\text { Heptane } \\
\text { Hydrocthloric Acid ( } 1.16 \mathrm{sp.} \mathrm{Gr.)} \\
\text { Isobutyl Alcohol (Isobutanol) } \\
\text { Isopropyl Alcohol } \\
\text { Methyl Accohol (Methanol, Wood Alcohol) } \\
\text { Methylene Chloride } \\
\text { Methyl Ethyl Ketone (MEK) } \\
\text { Methyl Isobutyl Ketone } \\
\text { Monoethanolamine (Ethanolamine) }\end{array}$ & $\begin{array}{c}25 \mathrm{~min} . \\
1 / 2 \mathrm{hr} . \\
6 \mathrm{hrs} . \\
2 \mathrm{hrs} . \\
2 \mathrm{hrs} .10 \mathrm{~min} . \\
6 \mathrm{hrs} . \\
6 \mathrm{~min} . \\
16 \mathrm{~min} . \\
1 / 2 \mathrm{hr} . \\
2 \mathrm{hrs} . \\
\end{array}$ & $\begin{array}{c}45 \\
30 \\
0 \\
0.75 \\
2 \\
0 \\
580 \\
120 \\
165 \\
1.3\end{array}$ & $\begin{array}{l}11 / 2 \mathrm{hrs} . \\
3 \mathrm{hrs} . \\
6 \mathrm{hrs} . \\
4 \mathrm{hrs} . \\
6 \mathrm{hrs} . \\
6 \mathrm{hrs} . \\
12 \mathrm{~min} . \\
1 / 4 \mathrm{hr} . \\
1 / 2 \mathrm{hr} . \\
5 \mathrm{hrs} .\end{array}$ & $\begin{array}{c}7 \\
3 \\
0 \\
0.8 \\
0 \\
0 \\
440 \\
101 \\
130 \\
0.7 \\
\end{array}$ & $\begin{array}{l}6 \mathrm{hrs} . \\
6 \mathrm{hrs} . \\
6 \mathrm{hrs} . \\
6 \mathrm{hrs} . \\
6 \mathrm{hrs} . \\
6 \mathrm{hrs} . \\
3 \mathrm{hrs} . \\
1 / 3 \mathrm{hr} . \\
12 / 3 \mathrm{hrs} . \\
6 \mathrm{hrs} .\end{array}$ & $\begin{array}{c}0 \\
0 \\
0 \\
0 \\
0 \\
0 \\
900 \\
82 \\
140 \\
0 \\
\end{array}$ & $\begin{array}{l}5 \mathrm{~min} . \\
6 \mathrm{~min} . \\
6 \mathrm{hrs} . \\
2 \mathrm{hrs} . \\
11 / 2 \mathrm{hrs} . \\
6 \mathrm{hrs} . \\
6 \mathrm{~min} . \\
6 \mathrm{~min} . \\
1 / 4 \mathrm{hr} . \\
41 / 2 \mathrm{hrs} .\end{array}$ & $\begin{array}{c}125 \\
117 \\
0 \\
0.75 \\
2.1 \\
0 \\
300 \\
170 \\
70 \\
1.1\end{array}$ & $\begin{array}{l}2 / 3 \mathrm{hr} . \\
3 / 4 \mathrm{hr} . \\
6 \mathrm{hrs} . \\
6 \mathrm{hrs} . \\
2 \mathrm{hrs} . \\
6 \mathrm{hrs} . \\
5 \mathrm{~min} . \\
7 \mathrm{~min} . \\
1 / 4 \mathrm{hr} . \\
6 \mathrm{hrs} .\end{array}$ & $\begin{array}{c}96 \\
83 \\
0 \\
0 \\
0.8 \\
0 \\
300 \\
120 \\
90 \\
0\end{array}$ \\
\hline $\begin{array}{l}\text { Nitric Acid (1.40 sp. Gr.) } \\
\text { N-Octane } \\
\text { Octyl Alcohol } \\
\text { Oxalic Acid (water saturated solution) } \\
\text { N-Pentane } \\
\text { Perchloric Acid (IN) } \\
\text { Perchloroethylene } \\
\text { Phenol } \\
\text { Phosphoric Acid (1.7 sp. Gr.) } \\
\text { Potassium Hydroxide ( } 30 \% \text { solution) }\end{array}$ & $\begin{array}{c}33 / 4 \mathrm{hrs} . \\
55 \mathrm{~min} . \\
6 \mathrm{hrs} . \\
6 \mathrm{hrs} . \\
1 / 3 \mathrm{hr} . \\
6 \mathrm{hrs} . \\
3 / 4 \mathrm{hr} . \\
11 / 3 \mathrm{hrs} . \\
6 \mathrm{hrs} . \\
6 \mathrm{hrs} .\end{array}$ & $\begin{array}{c}- \\
18 \\
0 \\
0 \\
35 \\
0 \\
19 \\
- \\
0 \\
0\end{array}$ & $\begin{array}{l}41 / 2 \mathrm{hrs} . \\
13 / 4 \mathrm{hrs} . \\
6 \mathrm{hrs} . \\
6 \mathrm{hrs} . \\
11 / 4 \mathrm{hrs} . \\
6 \mathrm{hrs} . \\
61 / 5 \mathrm{hrs} . \\
2 \mathrm{hrs} . \\
6 \mathrm{hrs} . \\
6 \mathrm{hrs} .\end{array}$ & $\begin{array}{c}- \\
12 \\
0 \\
0 \\
15 \\
0 \\
15 \\
- \\
0 \\
0\end{array}$ & $\begin{array}{l}4 \mathrm{hrs} . \\
6 \mathrm{hrs} . \\
6 \mathrm{hrs} . \\
6 \mathrm{hrs} . \\
6 \mathrm{hrs} . \\
6 \mathrm{hrs} . \\
4 \mathrm{hrs} . \\
2 / 3 \mathrm{hr} . \\
6 \mathrm{hrs} \\
6 \mathrm{hrs} .\end{array}$ & $\begin{array}{l}- \\
0 \\
0 \\
0 \\
0 \\
0 \\
1 \\
- \\
0 \\
0\end{array}$ & $\begin{array}{c}2 \mathrm{hrs} . \\
1 / 3 \mathrm{hr} . \\
3 / 4 \mathrm{hr} . \\
6 \mathrm{hrs} . \\
5 \mathrm{~min} . \\
6 \mathrm{hrs} . \\
6 \mathrm{~min} . \\
35 \mathrm{~min} . \\
6 \mathrm{hrs} . \\
11 / 3 \mathrm{hrs} .\end{array}$ & $\begin{array}{c}- \\
20 \\
1.7 \\
0 \\
152 \\
0 \\
100 \\
- \\
0 \\
-\end{array}$ & $\begin{array}{l}2 \mathrm{hrs} . \\
7 \mathrm{hrs} . \\
6 \mathrm{hrs} . \\
6 \mathrm{hrs} . \\
1 / 2 \mathrm{hr} . \\
6 \mathrm{hrs} . \\
7 \mathrm{~min} . \\
2 / 3 \mathrm{hr} . \\
6 \mathrm{hrs} . \\
3 \mathrm{hrs} .\end{array}$ & $\begin{array}{c}- \\
36 \\
0 \\
0 \\
111 \\
0 \\
95 \\
- \\
0 \\
-\end{array}$ \\
\hline $\begin{array}{l}\text { N-Propyl Alcohol (N-propanol) } \\
\text { Sodium Hydroxide ( } 50 \% \text { solution) } \\
\text { Sodium Hypochlorite ( } 30 \% \text { solution) } \\
\text { Sodiumhyposulfite (saturated solution) } \\
\text { Styrene } \\
\text { Sulfuric Acid ( } 1.83 \mathrm{sp} \text {. Gr.) } \\
\text { Toluene } \\
\text { Trichloroethylene } \\
\text { Xylene }\end{array}$ & $\begin{array}{c}2 \mathrm{hrs} . \\
6 \mathrm{hrs} . \\
6 \mathrm{hrs} . \\
6 \mathrm{hrs} . \\
1 / 3 \mathrm{hr} . \\
13 / 4 \mathrm{hrs} \\
1 / 2 \mathrm{hr} . \\
1 / 3 \mathrm{hr} . \\
2 / 3 \mathrm{hr} . \\
\end{array}$ & $\begin{array}{c}1.5 \\
0 \\
0 \\
0 \\
36 \\
- \\
71 \\
209 \\
62 \\
\end{array}$ & $\begin{array}{l}6 \mathrm{hrs} . \\
6 \mathrm{hrs} . \\
6 \mathrm{hrs} \\
6 \mathrm{hrs} \\
2 / 3 \mathrm{hr} . \\
4 \mathrm{hrs} \\
2 / 3 \mathrm{hr} \\
1 / 2 \mathrm{hr} . \\
3 / 4 \mathrm{hr} .\end{array}$ & $\begin{array}{c}0 \\
0 \\
0 \\
0 \\
31 \\
- \\
61 \\
207 \\
55 \\
\end{array}$ & $\begin{array}{l}6 \mathrm{hrs} . \\
6 \mathrm{hrs} . \\
6 \mathrm{hrs} . \\
6 \mathrm{hrs} . \\
1 / 2 \mathrm{hr} . \\
6 \mathrm{hrs} . \\
1 \mathrm{hr} . \\
1 / 3 \mathrm{hr} . \\
12 / 3 \mathrm{hr} .\end{array}$ & $\begin{array}{c}0 \\
0 \\
0 \\
0 \\
122 \\
0 \\
55 \\
184 \\
50 \\
\end{array}$ & $\begin{array}{l}11 / 6 \mathrm{hrs} . \\
6 \mathrm{hrs} . \\
6 \mathrm{hrs} \\
6 \mathrm{hrs} . \\
10 \mathrm{~min} . \\
11 / 3 \mathrm{hrs} . \\
9 \mathrm{~min} . \\
6 \mathrm{~min} . \\
7 \mathrm{~min} .\end{array}$ & $\begin{array}{c}1.5 \\
0 \\
0 \\
0 \\
58 \\
- \\
106 \\
210 \\
74 \\
\end{array}$ & $\begin{array}{l}11 / 2 \mathrm{hrs} . \\
6 \mathrm{hrs} . \\
6 \mathrm{hrs} . \\
6 \mathrm{hrs} . \\
1 / 5 \mathrm{hr} . \\
21 / 2 \mathrm{hrs} . \\
9 \mathrm{~min} . \\
8 \mathrm{~min} . \\
8 \mathrm{~min} .\end{array}$ & $\begin{array}{c}1 \\
0 \\
0 \\
0 \\
86 \\
- \\
83 \\
193 \\
68 \\
\end{array}$ \\
\hline
\end{tabular}


Chemical Resistance Selector Chart

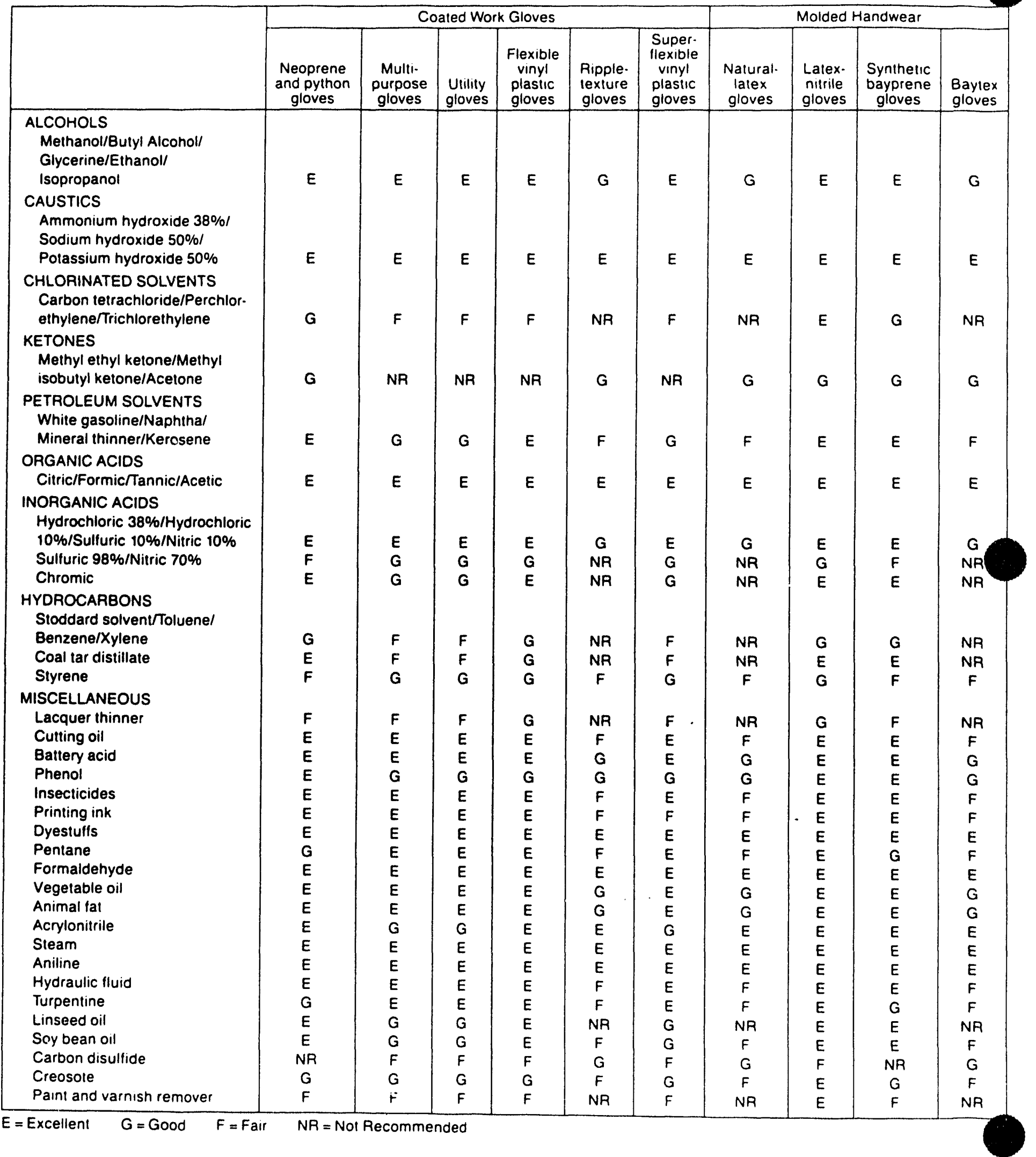




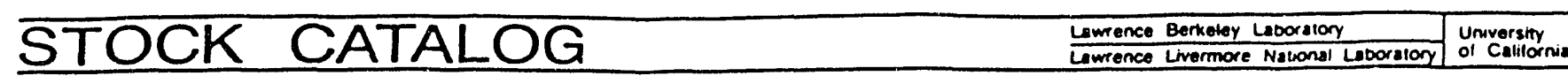

\begin{tabular}{|c|c|c|c|}
\hline Catalog No. & & Description & Uni! \\
\hline $\begin{array}{l}\text { COVERALL, } \\
2 \text { WAY } 2 I P E \\
\text { PANTS HAVE }\end{array}$ & $\begin{array}{l}\text { STEEL BLUE, } \\
\text { PER, } 2 \text { CHEST } \\
\text { 2 SIDE AND } \\
\text { SIZE }\end{array}$ & $\begin{array}{l}\text { DACRON, POLYESTER AND COTTON, } \\
\text { POCKETS, ELASTICIZED WAIST INSERTS, } \\
2 \text { BACK POCKETS, PLEATED BACK }\end{array}$ & \\
\hline $\begin{array}{l}8415-70616 \\
8415-70617 \\
8415-70618 \\
8415-70619 \\
8415-70620 \\
8415-70621 \\
8415-70622 \\
8415-70623\end{array}$ & $\begin{array}{l}40 L \\
42 S \\
42 R \\
42 L \\
44 R \\
44 L \\
46 R \\
46 I\end{array}$ & & $\begin{array}{l}\text { EA } \\
\text { EA } \\
\text { EA } \\
\text { EA } \\
\text { EA } \\
\text { EA } \\
\text { EA } \\
\text { EA }\end{array}$ \\
\hline
\end{tabular}

GLOVES, BUTYL RUBBER, SHOOTH, 14 IN. LONG, 32 MIL THICK, NORTH HAND PROTECTION NO. B-324

SIEE

$8415-70065$
$8415-70066$
$8415-70067$
$8415-70068$

GLoves, canVAs, cotron, heAVY, BOSS

SIRE OR. MPR. NO.

$\begin{array}{llllll}8415-28272 & \text { MENS LARGE } & 8 & 13021 & \text { PR }\end{array}$

$\begin{array}{lllll}8415-47819 & \text { MENS LARGE } 10 & 15021 & \text { PR }\end{array}$

8415-28273 GLOVES, CANVAS, LEATHER PALM, LARGE, BOSS 9565 PR

8415-66175 GLOVE, COTTON, 9 OL. NATURAL REVERSIBLE JERSEY, PR

CNN BE TURWED INSIDE OUT NWD WORN ON OPPOSITE

HAND, MENS INRGE, CENTURY GLOVE NO. FF8506H-I 8415-28274 GLOVES, COTTON, MITE, MORTICIANS, REVERSIBLE, PR
WARGE BIZE, CARDIHAL GLOVE GOS-L

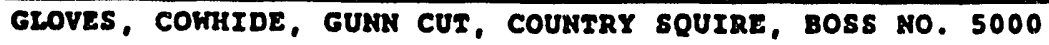

\begin{tabular}{|c|c|}
\hline $8415-58102$ & $\begin{array}{l}\text { MEDIUM } \\
\text { IARGE }\end{array}$ \\
\hline
\end{tabular}

GLOVES, DISPOSABLE LATEX, SURGEONS TYPE, CLASS 100 CLEANROOH

GRNDE, 50 PAIR PER PACKAGE, AMERICNN SCIENTIFIC PROD. MFR. SI8E MFR. Ho.

$\begin{array}{lllll}8415-68195 & 6 & G 7224-41 & \text { PG } \\ 8415-68196 & 7 & G 7224-43 & \text { PG } \\ 8415-68197 & 8 & G 7224-45 & \text { PG } \\ 8415-68198 & 9 & G 7224-47 & \text { PG }\end{array}$

GLOVES, DRY BOX, NEOPRENE GAUNTLET TYPE, 32 IN, LONG,

SIRE 9-3/4, NORTH CUFF IN.

INCHES THICK

KFR . NO.

$\begin{array}{lllll}8415-28307 & 5 & .015 & 5 N-1532-975 & P R \\ 8415-28306 & 5 & .030 & 5 N-3032-975 & \text { PR } \\ 8415-62373 & 5 & .015 & 5 N-1532-58-975 & \text { PR } \\ 8415-28308 & 8 & .015 & 8 N-1532-975 & \text { PR } \\ 8415-64593 & 8 & .015 & 8 N 1532 & \text { PR } \\ 8495-28303 & 8 & .030 & 8 N-3032-975 & \text { PR } \\ 8415-45714 & 7-8 & .015 & 7 N-1532-975 & \text { PR } \\ 8415-57874 & 7 & .030 & 7 N Y 3032 & \text { PR } \\ 8415-45713 & 8 & .030 & 8 N-3032-975 & \text { PR }\end{array}$

GLOVES, LATEX RUBBER, BUfF EMBOSSED, GLOVER SL-866 SIRE

8415-41576 8, MEDIUM

$8415-4157799^{\circ}$, LARGE

GLOVES, LATEX RUBBER, FLOCK LINED,

12 TO I4 IN. LENGTH, PLAYTEX

SIZE' MFR. NO

8415-28311 7. SHALL 834

8415-28312 8. MEDIUM 834

8415-28313 9. LARGE 834L 


\begin{tabular}{|l|l|l|l|}
\hline Catalog No. & Description & Unit \\
\hline
\end{tabular}

GLOVES, LATEX RUBBER, FLOCK LINED,
12 TO I IN. LENGTH, PLAYTEX
SIZE

8415-49806 10, EXTRA LARGE $834 \times$

GLOVES, LEATHER, CREAM GRAIN COWHIDE, NAPA GLOVE CO. SIRE

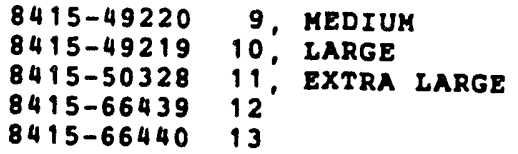

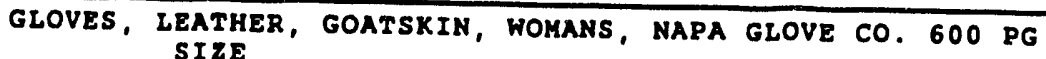

8415-64074 8

$8415-64075 \quad 9$

PR GLOVES, MILLED NEOPRENE, HEAVY DUTY, NON-SLIP GRIP
SIZE
MFR./NO. LONG

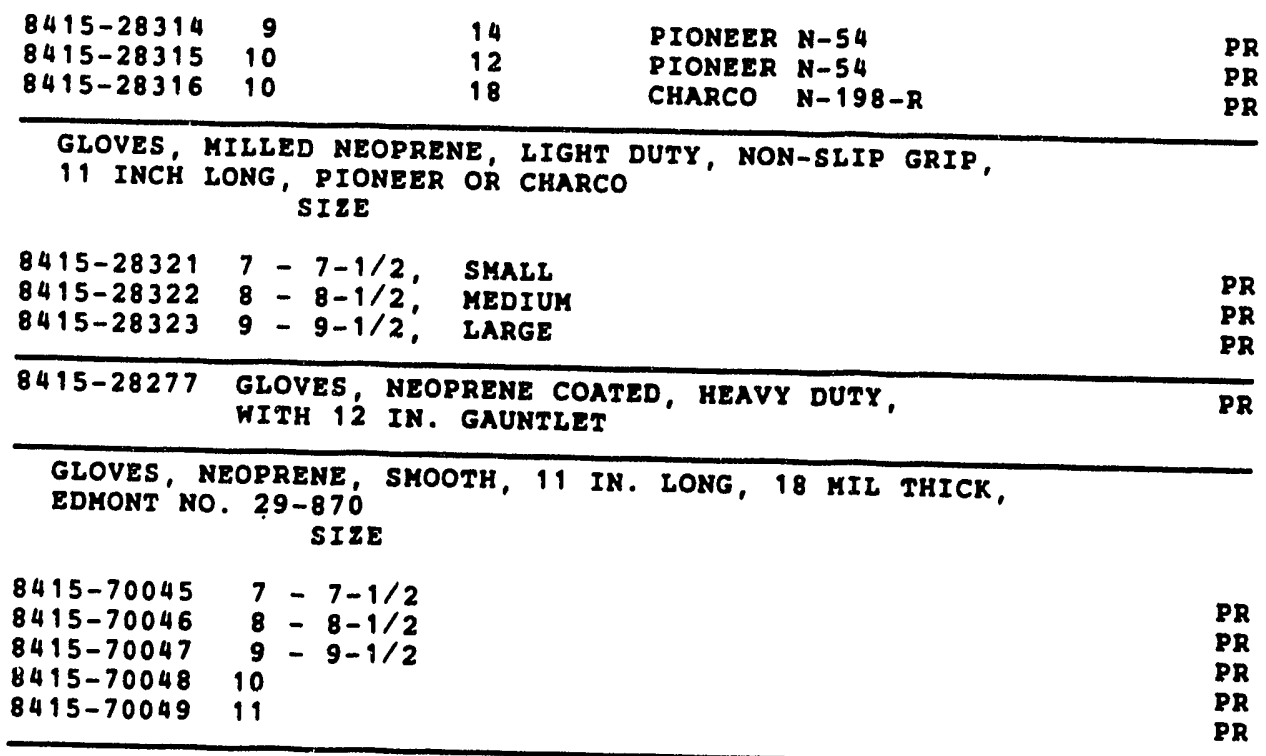

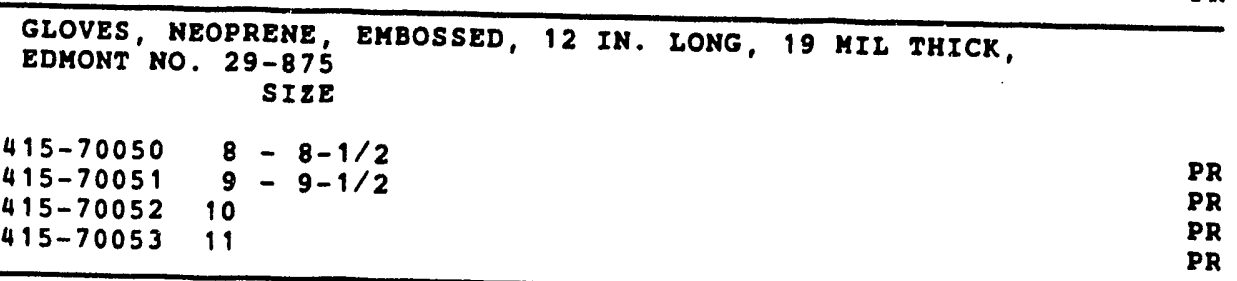
GLOVES, NITRILE, EMBOSSED, 15 IN. LONG, 22 MIL THICK,
EDMONT NO. $37-165$

$$
\text { SIZE }
$$

$8415-70054$

$8415-70055$

$8415-70056 \quad 9-9-1 / 2$

$8415-70057 \quad 10$

$8415-70058 \quad 11$

8415-56208 GLOVES, PLASTIC, TEXTURED NONSTERTLE SR LARGE SIZE, 50/BOX, TOMAC'NO. 22798-060

GLOVES, POLYETHYLENE, DISPOSABLE, W/LONG WRIST PROTECTION

$100 / B O X$, WILLSON "WILGARD" NO. 35-112 (FOR EITHER HAND)

$8415-28282$ SHALL

$8415-28283$ MEDIUM

$8415-28284$ LARGE 


\section{STOCK CATALOG}

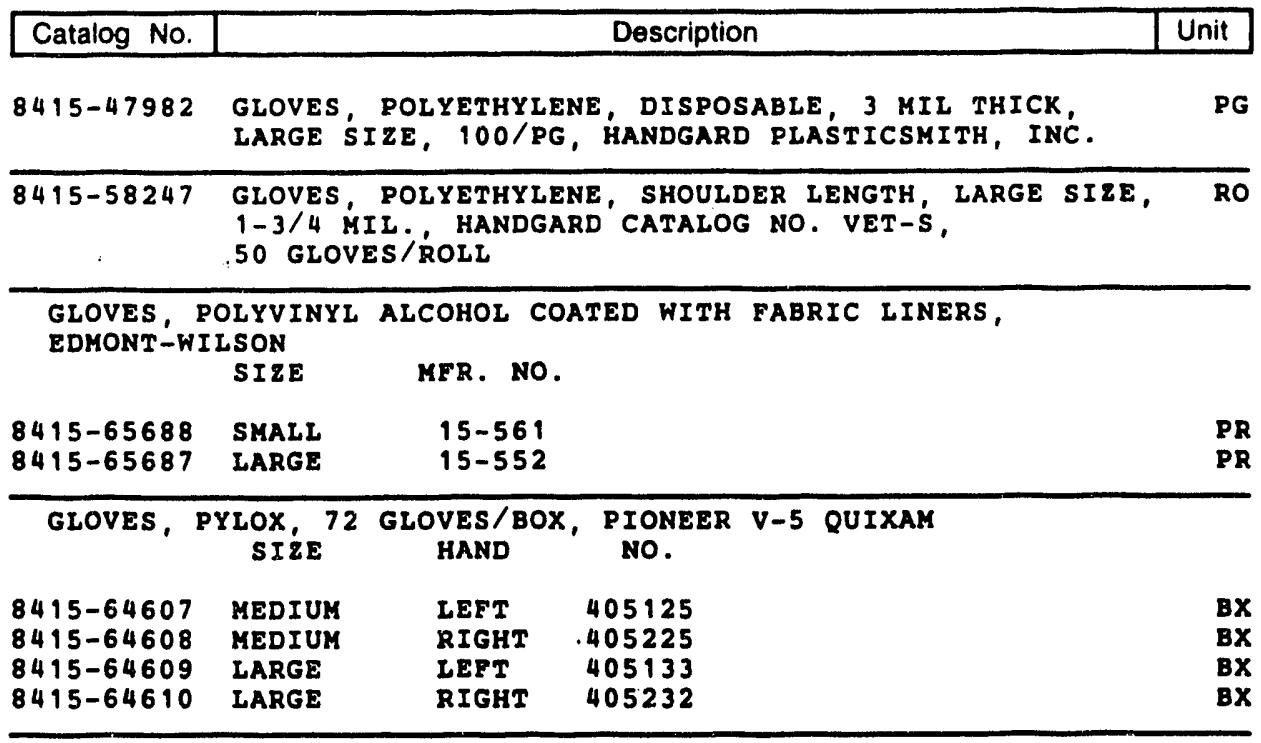

GLOVES, PYLOX, SHEER WEIGHT, TISSUE THIN,

$10-1 / 2$ IN. LONG, PIONEER MODEL V-10 SIZE

8415-28285 MEDIUY

PR 8415-28286

LARGE

PR

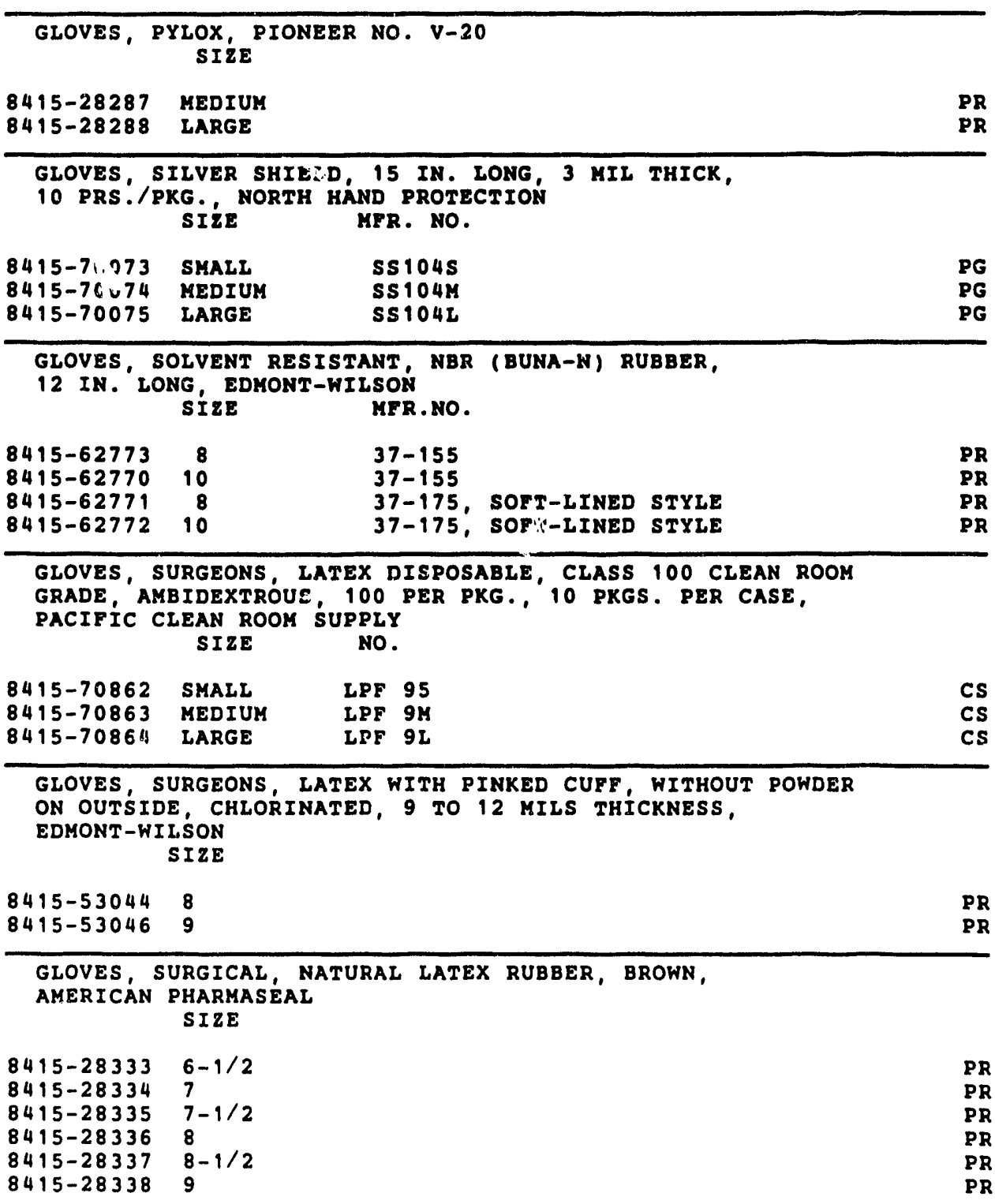




\begin{tabular}{|c|c|c|}
\hline Catalog No. & Description & Unit \\
\hline $8495-68206$ & $\begin{array}{l}\text { GLOVES, SURGICAL, RUBBER, SIZE 6, TRAVENOL } 207151 \text {, } \\
\text { AMERICAN PHARMASEAL NO. P8060 }\end{array}$ & PF \\
\hline GLOVES , & $\begin{array}{l}\text { YNTHETIC RUBBER, SURETY } \\
\text { WEIGHT SIZE }\end{array}$ & \\
\hline $\begin{array}{l}8415-28318 \\
8415-28319 \\
8415-28320\end{array}$ & $\begin{array}{l}\text { LIGHT } \\
\text { MEDIUM } \\
\text { MEDIUH }\end{array}$ & $\begin{array}{l}\text { PF } \\
\text { PF }\end{array}$ \\
\hline $\begin{array}{l}\text { GLOVES, } \\
18 \mathrm{KIL}, \mathrm{TI}\end{array}$ & $\begin{array}{l}\text { NSUPPORTED RUBBER, EMBOSSED, } 13 \text { IN. LONG, } \\
\text { ICX, EDMONT NO. } 36-124 \\
\text { SIRE }\end{array}$ & \\
\hline $\begin{array}{l}8415-70060 \\
8415-70061 \\
8415-70062 \\
8415-70063 \\
8415-70064\end{array}$ & $\begin{array}{r}7-7-1 / 2 \\
8-8-1 / 2 \\
9-9-1 / 2 \\
10 \\
11\end{array}$ & $\begin{array}{l}\text { PF } \\
P F \\
P F \\
P F \\
P F\end{array}$ \\
\hline $8415-70059$ & $\begin{array}{l}\text { GLOVES, VINYL COATED, HEAVY DUTY PVC, } 14 \text { IN. LONG, } \\
\text { MENS SIZE, EDHONT NO: } 24-364\end{array}$ & PR \\
\hline GLOVES, & $\begin{array}{l}\text { IAYL DISPOSABLE, } 50 \text { PR/BAG, OAK RUBBER } 96 \\
\text { SIRE }\end{array}$ & \\
\hline $\begin{array}{l}8415-64993 \\
8415-64994 \\
8415-64995\end{array}$ & $\begin{array}{l}\text { SMALL } \\
\text { MEDIUM } \\
\text { LARGE }\end{array}$ & $\begin{array}{l}\text { Py } \\
\text { PI }\end{array}$ \\
\hline GLOVES, V & $\begin{array}{l}\text { INYL IMPREGNATED COTTON, EDHONT-WILSON } \\
\text { SIRE } \\
\text { MPR. NO. }\end{array}$ & \\
\hline $\begin{array}{l}8415-43820 \\
8415-28275 \\
8415-28276\end{array}$ & $\begin{array}{lll}\text { MENS } & \text { MEDIUH } & 51-327 \\
\text { MENS } & \text { LARGE } & 51-327 \\
\text { WONENS MEDIUY } & 51-321\end{array}$ & $\begin{array}{l}\text { PE } \\
\text { PE } \\
\text { PE }\end{array}$ \\
\hline $\begin{array}{l}\text { GLOVES, V } \\
\text { WITH ELAS }\end{array}$ & $\begin{array}{l}\text { CNYL IMPREGNATED COTTON, FOAM INSULATED, } \\
\text { CIC WRIST, EDHONT-WILSON } 31-791 \\
\text { SIZE }\end{array}$ & \\
\hline $\begin{array}{l}8415-57262 \\
8415-57263\end{array}$ & $\begin{array}{l}\text { MEDIUK } \\
\text { IARGE }\end{array}$ & $\begin{array}{l}\text { PR } \\
\text { PR }\end{array}$ \\
\hline
\end{tabular}

GLOVES, VITON MATERIAL, FOR KANDLING PCB AND OTHER TOXIC MATERIALS, 10 MIL THICK, 11 IN, LONG, NORTON NO. F-101

$\begin{array}{llll}8415-69377 & 8 & & \\ 8415-69378 & 9 & P R \\ 8415-69379 & 10 & & P R \\ P R\end{array}$

GLOVES, WELDING

\begin{tabular}{|c|c|}
\hline $\begin{array}{l}8415-68055 \\
8415-28279 \\
8415-28280 \\
8415-51008\end{array}$ & $\begin{array}{l}\text { ELK HIDE LEATHER, LARGE SIZE, NAPA GLOVE NO. } 67 \text { WT } \\
\text { SPLIT LEATHER, SIZE } 10, \text { STEEL GRIP SC } 13475 \\
\text { LEATHER, CLUTE PATTERN, GAUNTLET CUFE, } \\
\text { SIZE 10-1/2, NO. } 1101 \\
\text { TERRI-CORD, HEAT AND FLAME RESISTANT }\end{array}$ \\
\hline $8415-70680$ & $\begin{array}{l}\text { GLOVE, ZETEX, HEAT RESISTANT, NON-ASBESTOS, } \\
\text { I4 IN. LINED WITH } 10 \text { OZ. WOOL, SARGENT WELCH } C O \text {. } \\
\text { NO. S-40257-10 }\end{array}$ \\
\hline $8415-71339$ & $\begin{array}{l}\text { GLOVE, ZETEX PLUS, HEAT RESISTANT, NON-ASBESTOS, } \\
14 \text { IN. NEWTEX INDUSTRIES, INC. NO. } 20112-1400-2 \mathrm{P} \\
\text { (THESE' ARE TO REPLACE } 8415-70680 \text { ) }\end{array}$ \\
\hline
\end{tabular}




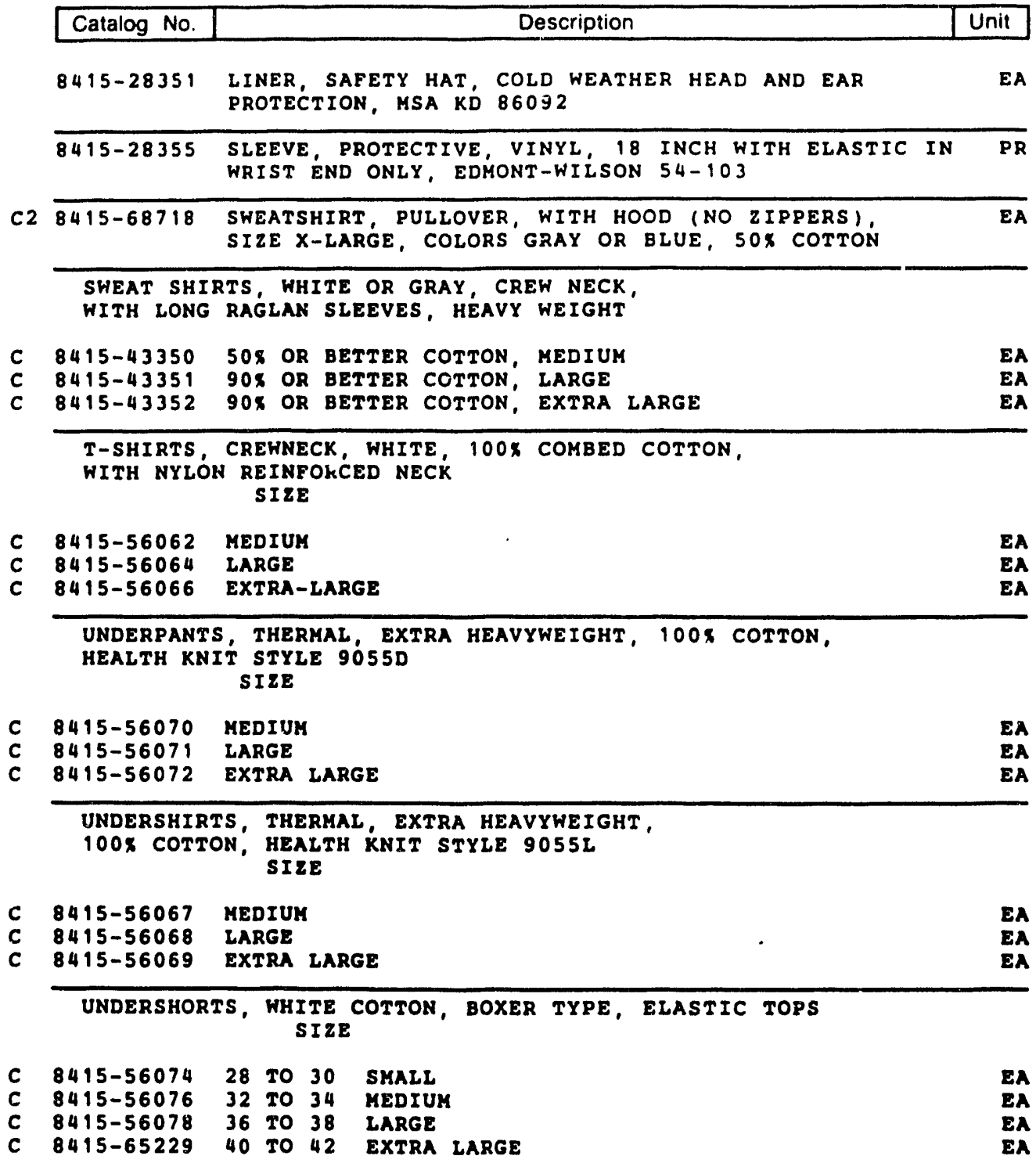

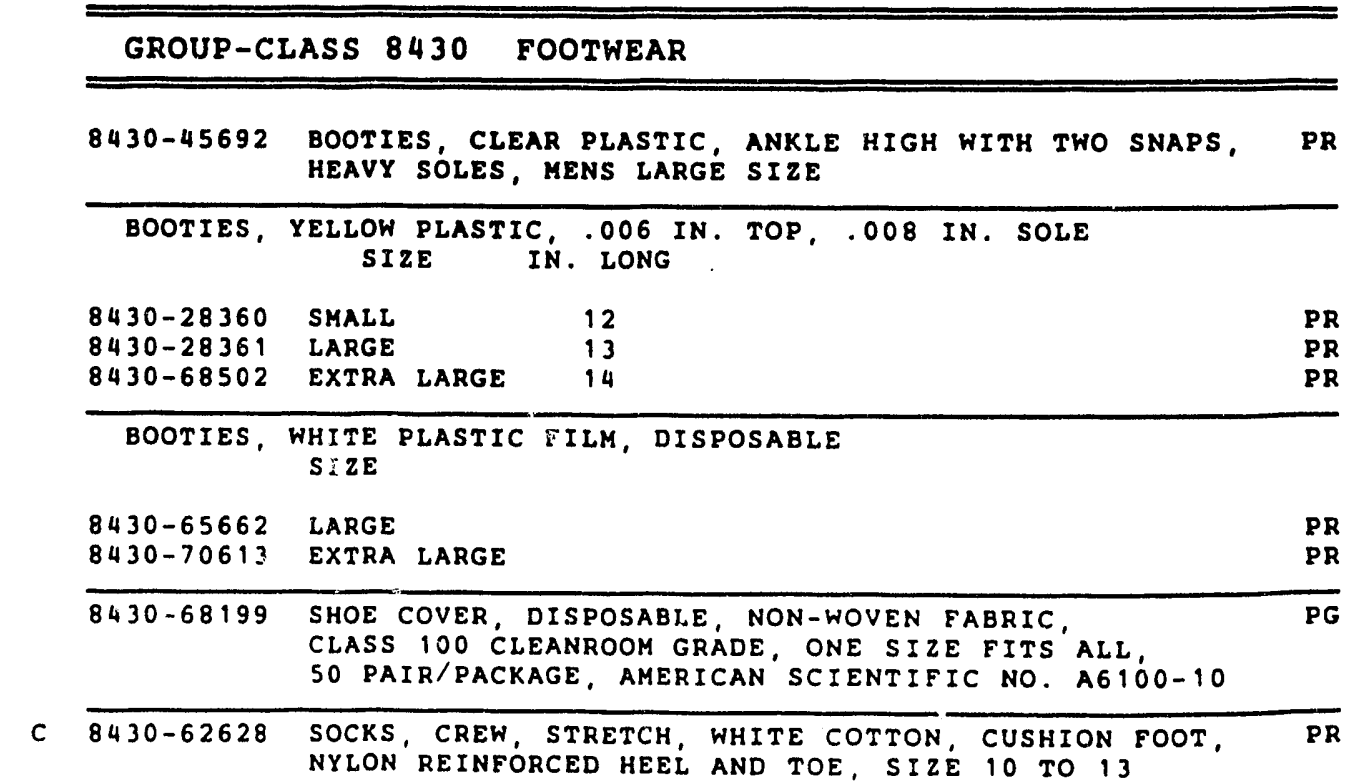




\section{Appendix 7}

\section{Labeling Information}




\section{NATIONAL FIRE PROTECTION ASSOCIATION (NFPA) LABELS}

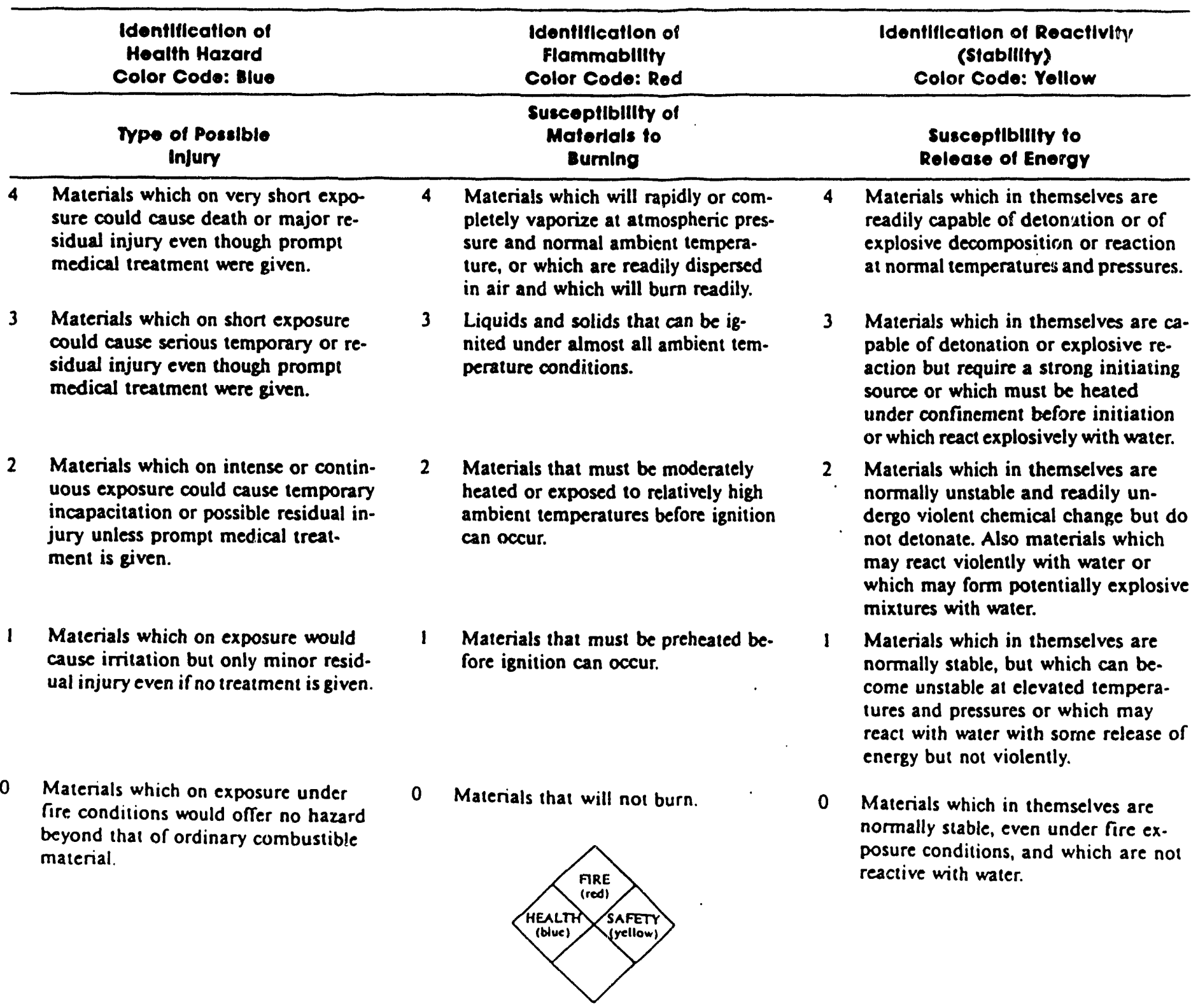




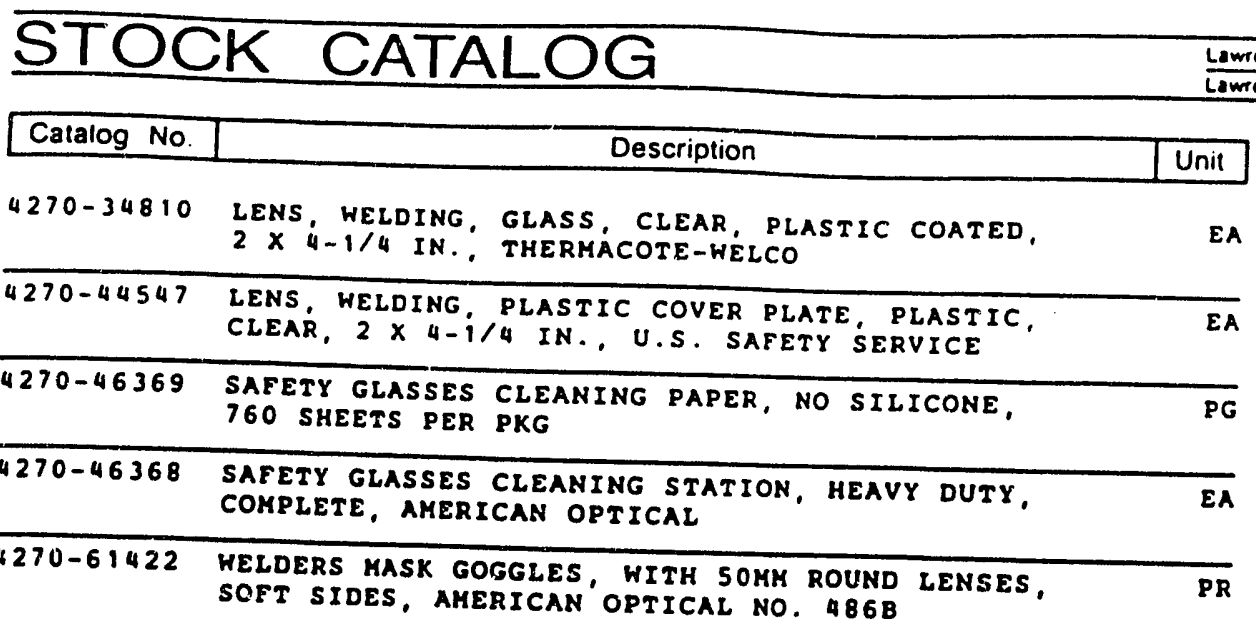

GROUP-CLASS 4280 SAFETY LABELS, SIGNS AND TAGS

LAB SAFETY SUPPLY

\section{LEGEND}

$4280-71381$

$4280-71382$

$4280-71383$

$4280-71384$

$4280-71385$

$4280-71386$

$4280-71387$

4280-71388

$4280-71389$

4280-71390

4280-7139

$4280-71392$

$4280-71393$

$4280-71394$

$4280-71395$

4280-71396

4280-71397

$4280-71398$

$4280-71399$

4280-71400

4280-71401

$4280-71402$

$4280-71403$

$4280-71404$

$4280-71405$

4280-71406

$4280-71407$

$4280-71408$

$4280-71409$

$4280-71410$

$4280-67348$
AUTHORI ZED PERSONNEL ONLY

DO NOT ENTER

NO SMOKIHG

HEAR SAFETY GOGGLES

HO EATING OR DRINKING

KEEP OUT

hEAR FACE PROTECTION

RESPIRATOR REQUIRED

WEAR SAFETY GLASSES

CORROSIVE MATERIALS

TOXIC CHEKICALS

FLAMMABLE SOLVENTS

hazARE RATING

HIGH VOLTAGE

ELECTRICAL HALARD

ULTRAVIOLET LIGHT

EXPLOSIVE

MICROHAVE RADIATION

LASER LIGHT

HEAR PROTECTIVE CLOTHIAG

INFECTIOUS AGENT

BIOHARARD

CAMCER hazARD

RADIOACTIVE MATERIAL

PROTECTIVE EQUIPMENT, HAZARD RATING

RADIATION HAZARD

LABORATORY ANIMALS

EAR PROTECTION REOUIREED

CHEMICAL STORAGE AREA

NUMBER

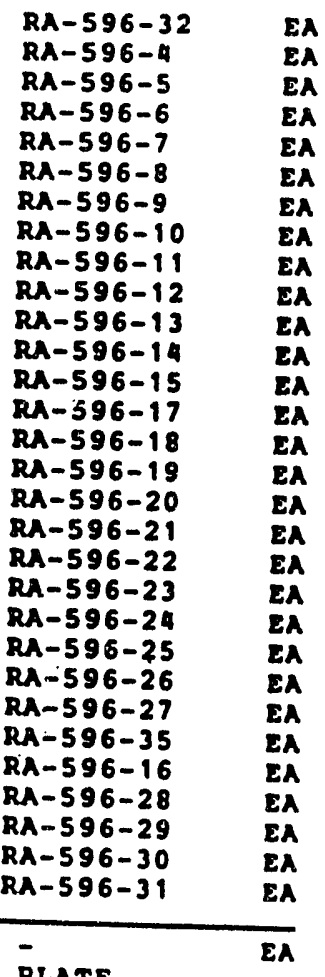

LABEL, HAZARD CONTROL, (H-C HOOD SURVEY -

SATISFACTORY - UP TO - INCHES FROM FACE PLATE -

WATER/WEATHER SELF-STICKING, WRITE-ON SURFACE

YELLOW, APPROX. $3-1 \% 2$ BLACK ON WHITE OR BLACK ON

LABEL, HAZARDOUS AND RADIOACTIVE MIXED HASTE.

INCHES

4280-70977

$4280-70978$

$6 \times 6$

$6 \times 6$, SITE 300 ONLY, 100 PER ROLL EA

$4280-71357$

LABEL, HAZARD IDENTITY WRITE-ON, IDENTIFY EA

CHEMICALS WITH NAME OWNER DATE IDENTIFY

RATING, $1-1 / 2 \times 3-7 / 8$ OWNER, DATE AND HAZARD

LAB SAFETY RA-706 


\begin{tabular}{|l|l|l|}
\hline Catalog No. & Description & Unit \\
\hline
\end{tabular}

LABEL, HAZARD RATING CHEMICAL, COLOR-CODED, FILL IN

INFORKATION, SELF-ADHESIVE, 100 PER PAD, LAB SAFETY IN. No.

4280-71352 $2 \times 2 \quad$ RA-55

$4280-713534 \times 4 \quad R A-553$

PD

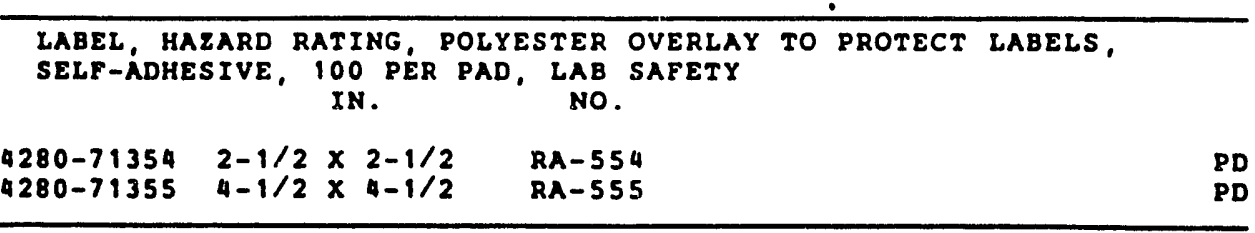

LABEL, HAZARDOUS RETENTION TANK WASTE, BLACK ON YELLOW, TYVEK MATERIAL, 200 PER ROLL

INCHES

$4280-709896 \times 6$

4280-711136 6 6, SITE 300 ONLY, 100 PER ROLL EA

4280-709827× $7 i \quad$ EA

LABEL, HAEARDOUS HASTE, RED AND WHITE,

TYVEK MATERIAL, 200 PER ROLL INCHES

$4280-709836 \times 6$

$4280-711106 \times 6$, SITE 300 ONLY, 100 PER ROLL

4280-709847× 7 i EA

LABEL, RADIOACTIVE WASTE, BLACK ON YELLOW,

TYVEX MATERIAL, 200 PER ROLI

INCHES

$4280-709796 \times 6$

4280-711126 6 6, SITE 300 ONLY, 100 PEX ROLL EA

$\begin{array}{llll}4230-70980 & 7 \times 11 & \end{array}$

IABEL, SAFETY, CARCINOGENIC HARARDS, PRESSURE SENSITIVE.

BLACX IEGEND ON RED BACKGROUND, $100^{\circ}$ PER ROLL

$\begin{array}{llll}\text { 4280-64467 DANGER CARCINOGENIC WASTE, } 5 \times 6 \text { IN. } & \text { RO } \\ \text { 4280-64462 DANGER CHEMICKI CARCINOGEN, } 1 \times 3 \text { IN. } & \text { RO }\end{array}$

IABEL, SAFETY, CAUTION, PRESSURE SENSITIVE, HATER-PROOF,

BLACX LEGEND ON YELLOH BACKGROUND, $7 \times 4$ INCH

4280-69388 A-9 ALUKINUK CUTTING TOOL FLUID-D-

4280-69389 COOL-TOOL CUTTING OII--

4280-69958 LABEL, SAFETY, CAUTION, PRESSURE SENSITIVE EA

ADHESIVE, IEGEND (CAUTION - HOT SUPFACE)

BLACK LETTERS ON YELLOW BACKGROUND

.002 IN. THICK BRIGHT SIIVER MAYLAR.

$2-1 / 2 \times 3-1 / 2$ INCHES

LABEL, SAFETY CAUTION, PRESSURE SENSITIVE ADHESTVE,
CAUTION - IN YELLON LETTERS ON BLACK BACKGROUND
LEGEND - IN BLACK LETTERS ON YELLON BACKGROUND

SIZE-IN. LEGEND - CAUTION

4280-69974 $1.5 \times 1$ - MULTIPIE POHER SOURCES TEST PG

4280-67194 $2 \times 3$ FOR ALI VOLTAGES, $25 /$ PKG.

- WEAR EYE PROTECTION HHTLE OPERATING EA

$4280-6709$

$3 x$

- DO NOT STORE FOOD OR BEVERAGE IN THIS REFRIGERATOR

$4280-6709$

$3 \times 5$ - EQUIPMENT CONTAINS PCB

$\begin{array}{lll}4280-64646 & 3 \times 5 & - \\ 4280-67088 & 4 \times 5-1 / 2 & \text { (LEGEND SPACE BI.ANK) }\end{array}$

$4280-67088$

$4280-6708$

$4 \times 5-1 / 2$

$4 \times 7$

$4280-67080$

$4 \times 7$

- ACID WASTE ONLY

$4280-67079$

$4 \times 7$

$4280-69975$

$4.5 \times 3$

- ORGANIC HASTE SOLVENTS ONLY

- MULTIPLE POHER SOURCES TEST FOR ALL VOLTAGES, $25 /$ PKG.

4280-64647 $7 \times 10$ - (LEGEND SPACE BLÁNK)

PG

EA

EA

EA

EA

EA

EA

EA

PG

EA 
LABEL, SAFETY, CAUTION, LEGEND - "DO NOT TURN OFF/ON-OFF",

SELF-ADHERING PAPERBOARD, COLOR ORANGE INCHES

\begin{tabular}{lll}
$4280-34900$ & $1-1 / 8 \times 4-5 / 8$ & \\
$4280-50172$ & $2-1 / 2 \times 5-1 / 4$ & EA \\
\hline $4280-67090$ & $\begin{array}{l}\text { LABEL, SAFETY, CHEMICAL HAZARDS, SELF-ADHERING, } \\
\text { LEGEND NON-PCB, } \\
\text { BACKGROUND }\end{array}$ & EA IN, WHITE LEGEND ON BLUE \\
\hline
\end{tabular}

LABEL, SAFETY, CHEMICAL HAZARDS, SELF-ADHERING,

CAUTIÓN - CONTAINS POLYCHLORINATED BIPHENYLS (ṔCBS), HCL CO. INCH

\begin{tabular}{lll}
$4280-64776$ & $1 \times 2$ & EA \\
$4280-64775$ & $6 \times 6$ & EA \\
\hline
\end{tabular}

4280-64775 $6 \times 6$ EA

LABEL, SAFETY, CHEKICAL HAZARDS, HATERPROOF PLASTIC COATED

FABRIC MATERIÁ, SELF-ADHERING, BLACK LEGEND ON

YELLOW BACKGROUND SIRE/IN.

LEGEND

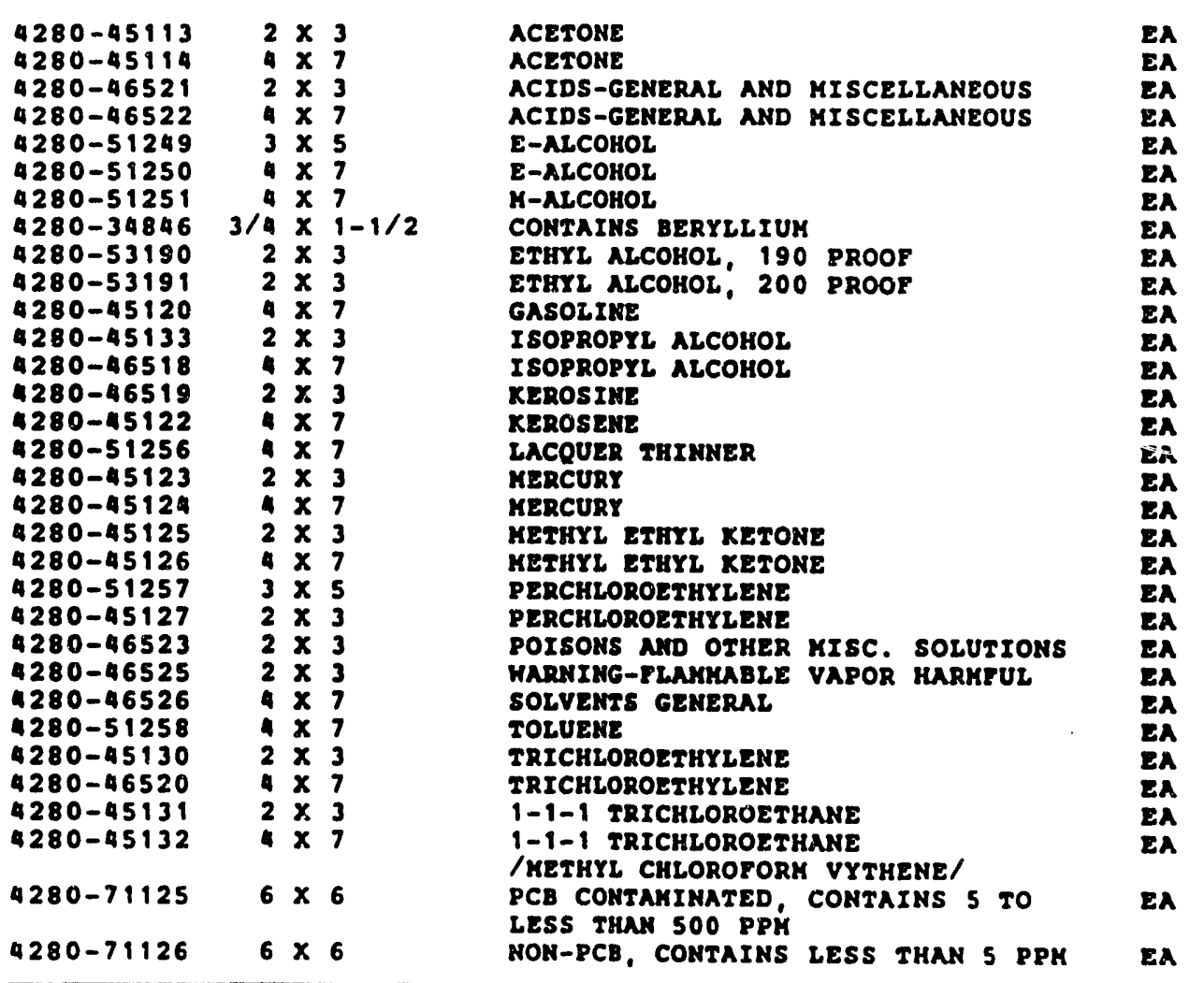

LABEL, SAFETY CHEMICAL HAZARDS, HATER PROOF PLASTIC COATED

SELF-ÁDHERING, BLACK LEGEND ON YELLOH BACK GROUND LEGEND

$\begin{array}{lllll}4280-67676 & \text { FLUOROCARBON TF SOLVENT } & 2 \times 3 & \text { EA } \\ 4280-67677 & \text { FLUOROCARBON TF SOLVENT } & 4 \times 7 & \text { EA }\end{array}$

LABEL, SAEETY, CHEMICAL HAZARDS H/SYMBOL, PLASTIC COATED,

SELF-ADHERING, BLACK LEGEND ON YELLOW BACKGROUND

$$
\text { LEGEND IN. }
$$

\begin{tabular}{llll}
$4280-45117$ & BENZENE & $2 \times 3$ & EA \\
$4280-45118$ & BERYLLIUM & $2 \times 3$ & EA \\
$4280-45119$ & CARBON TETRACHLORIDE & $2 \times 3$ & EA \\
\hline $4280-67347$ & LABEL, SAFETY, DANGER, PRESSURE SENSITIVE, & EA \\
& $\begin{array}{l}\text { WATER-PROOF, HRITEABLE (DANGER - CANCER-SUSPECT } \\
\text { AGENT, DATE - COMPOUND - CONCENTRATION), DANGER - }\end{array}$ \\
& IN HHITE LETTERS IN RED OVAL FIELD ON BLACK \\
& BACKGROUND, LEGEND IN BLACK LETTERS ON HHITE \\
& BACKGROUND, I X 3.IN.
\end{tabular}




\begin{tabular}{|l|l|l|}
\hline Catalog No. & Description & Unit \\
\hline
\end{tabular}

LABEL, SAFETY, DANGER, FOR SWITCHES, SELF-ADHERING PAPERBOARD, UPPER LEGEND-DANGER, IN WHITE LETTERS ON RED FIELD ON BLACK BACKGROUND, LOWER LEGEND-DO NOT TURN ON, IN BLACK LETTERS ON WHITE BACKGROUND. INCH

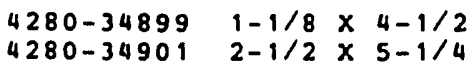

LABEL SAFETY DANGER PRESSURE SENSTTIVE ADHESTVE DANGER - IN WHITE LETTERS IN RED OVAL FIELD ON BLACK

LABEL, SAFETY, GENERAL PURPOSE, SELF-ADHERING VINYL PLASTIC,

PER USASI SPEC 853.1 NND 835.1 , THE MABI CO.

SI\&E-INCHES

QUANTITY

PER SHT

$\begin{array}{lll}4280-57575 & 1-1 / 8 \times 4-1 / 2 \\ 4280-57576 & 1-1 / 8 \times 4-1 / 2 \\ 4280-57577 & 1-1 / 8 \times 4-1 / 2 \\ 4280-57578 & 1-1 / 8 \times 4-1 / 2 \\ 4280-57574 & 2-1 / 4 \times 4-1 / 2 \\ 4280-57579 & 2-1 / 4 \times 9\end{array}$

\section{IEGEND}

$\begin{array}{lll}4 & 120 \text { VOLTS } & \text { SH } \\ 4 & 208 \text { VOLTS } & \text { SH } \\ 4 & 240 \text { VOLTS } & \text { SH } \\ 4 & 480 \text { VOLTS } & \text { SH } \\ 2 & \text { DANGER HIGH VOLTAGE } & \text { SH } \\ 1 & \text { DOOR INTERLOCKED } & \text { SH }\end{array}$

EA

EA

EA

5A

BA

EA

EA

EA

2A

IABEL, SAFETY, GENERAL PURPOSE, SELF-ADHERING VINYI PIASTIC,

PER USASI SPEC 253.1 AND 235.1; THE MABI CO., 50 PER ROLI

SIZE-IN. LEGEND

$\begin{array}{rrlll}4280-57569 & 1-1 / 2 \times 3 & \text { DANGER HIGH VOLTAGE } & \text { RO } \\ 4280-57570 & 5 / 8 \times 3 & 120 \text { VOLTS } & \text { RO } \\ 4280-57571 & 5 / 8 \times 3 & 208 \text { VOLTS } & \text { RO } \\ 4280-57572 & 5 / 8 \times 3 & 240 \text { VOLTS } & \text { RO } \\ 4280-57573 & 5 / 8 \times 3 & 480 \text { VOLTS } & \text { RO }\end{array}$

LABEL, SAFETY, NOTICE, PRESSURE SENSITIVE ADHESIVE,

NOTICE - IN WHITE LETTERS ON BLUE BACKGROUND

LEGEND - IN BLACK LETTERS ON WHITE BACKGROUND

SIZE-IN. LEGEND - NOTICE

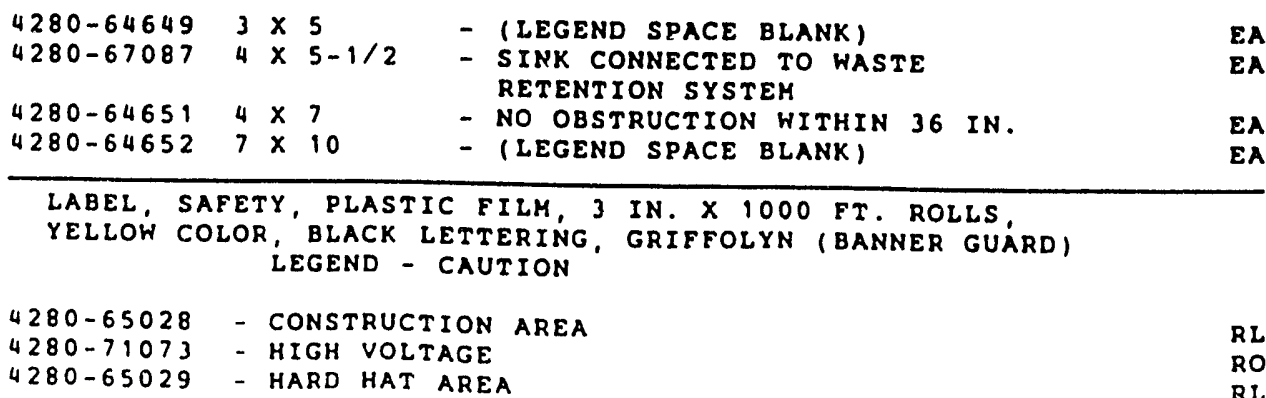

- HARD HAT AREA RI 


\section{STOCK CATALOG}

\begin{tabular}{|c|c|c|}
\hline Catalog No & Description & Unit \\
\hline $4280-70084$ & $\begin{array}{l}\text { LABEL, SAFETY, HAZARDOUS WASTE, SELF-ADHESIVE, } \\
6 \times 6 \text { INCHES, VINYL WITH ULTRA VIOLET INK TO } \\
\text { GUARD AGAINST FADING, } 2 \text {-COLOR, DARK RED AND BLACK } \\
\text { LETTERING ON WHITE BACKGROUND }\end{array}$ & EA \\
\hline $4280-56938$ & $\begin{array}{l}\text { LABEL, SAFETY, HIGH VOLTAGE, PRESSURE SENSITIVE, } \\
\text { STANDARD COLOR - LETTERS IN RED ON WHITE AND BLACK } \\
\text { BACKGROUND. LETTERS I IN. HIGH SIZE } 5-1 / 4 \times 2-1 / 2 \\
\text { IN. 100 PER PAD, THE MABI CO. }\end{array}$ & PD \\
\hline $4280-70085$ & $\begin{array}{l}\text { LABEL, SAFETY, WASTE CONTINUATION, SELF-ADHESIVE, } \\
6 \text { X } 6 \text { INCHES, VINYL WITH ULTRA VIOLET INK TO GUARD } \\
\text { AGAINST FADING, } 1 \text {-COLOR, BLACK LETTERS ON WHITE } \\
\text { BACKGROUND }\end{array}$ & EA \\
\hline $4280-67086$ & $\begin{array}{l}\text { LABEL, SAFETY, PRESSURE SENSITIVE, ARROWS } \\
\text { (DIRECTIONAL); } 1 / 4 \times 1 \text { IN. BLACK ARROWS ON YELLOW } \\
\text { BACKGROUND, } 81 \text { ARROWS PER SHEET }\end{array}$ & $\mathrm{SH}$ \\
\hline $4280-70758$ & $\begin{array}{l}\text { LABEL, PRESSURE SENSITIVE, LEGEND - DO NOT RETURN } \\
\text { THIS CHEMICAL TO STORES - YELLOW BACKGROUND WITH } \\
\text { BLACK LETTERING, } 1 \text { IN. HIGH } X 2 \text { IN. LONG, 100/RO }\end{array}$ & RO \\
\hline $4280-67084$ & $\begin{array}{l}\text { LABEL, SAFETY, PRESSURE SENSITIVE, } 1-1 / 2 \times 3-5 / 8 \\
\text { IN. LEGEND H.C. HOOD SURVEY UNSATISFACTORY, BLACK } \\
\text { ON WHITE AND YELLOW BACKGROUND }\end{array}$ & EA \\
\hline $4280-67083$ & $\begin{array}{l}\text { LABEL, SAFETY, PRESSURE SENSITIVE, } 3 \text { X } 3-1 / 2 \text { IN., } \\
\text { LEGEND H.C. HOOD SURVEY - SATISFACTORY AVERAGE } \\
\text { FACE VELOCITY. BLACK ON WHITE BACKGROUND, } 3 \text { LABELS } \\
\text { PER SHEET }\end{array}$ & SH \\
\hline $4280-67082$ & $\begin{array}{l}\text { LABEL, SAFETY, PRESSURE SENSITIVE, } 4-1 / 2 \times 3-1 / 2 \\
\text { IN. LEGEND H.L. HOOD SURVEY - SATISFACTORY UNDER } \\
\text { FOLLOWING CONDITIONS, BLACK ON WHITE BACKGROUND, } 2 \\
\text { LABELS PER SHEET }\end{array}$ & SH \\
\hline $4280-67085$ & $\begin{array}{l}\text { LABEL, SAFETY, PRESSURE SENSITIVE, } 4-1 / 2 \times 3-1 / 2 \\
\text { IN. LEGEND H.C. HOOD SURVEY - SATISFACTORY UNDER } \\
\text { FOLLOWING CONDITIONS, BLACK ON YELLOW BACKGROUND, } \\
2 \text { LABELS PER SHEET }\end{array}$ & SH \\
\hline $4280-56959$ & $\begin{array}{l}\text { LABEL, SAFETY, RADIOACTIVE MATERIAL ISOTOPE, WITH } \\
\text { RADIATION SYMBOL, ATOMIC PRODUCTS CORP., N.Y., } \\
\text { CAT. NO. WT- } 12, i \times 2 \text { IN. }\end{array}$ & RO \\
\hline $4280-56957$ & $\begin{array}{l}\text { LABEL, SAFETY RADIOACTIVE MATERIAL, LEGEND - } \\
\text { CAUTION CONTAMINATION HAZARD - DO NOT TOUCH - } \\
\text { RADIOACTIVE MATERIAL - WITH RADIATION SYMBOL, } \\
2-1 / 4 \times 9 \text { IN. }\end{array}$ & EA \\
\hline $\begin{array}{l}\text { LABEL, SAF } \\
\text { SUN AND RA }\end{array}$ & $\begin{array}{l}\text { FETY, SELF-ADHERING, VINYL PLASTIC, } \\
\text { AIN RESISTANT, PERMANENT COLORS } \\
\text { SI } 2 E-I N . \\
\text { LEGEND }\end{array}$ & \\
\hline $\begin{array}{l}4280-58027 \\
4280-58028 \\
4280-58029 \\
4280-58031 \\
4280-58035 \\
\end{array}$ & $\begin{array}{lll}10 & \times 14 & \text { EXIT } \\
14 & \times 6-1 / 2 & \text { EXIT (WITH ARROW LEFT) } \\
14 & \times 6-1 / 2 & \text { EXIT (WITH ARROW RIGHT) } \\
14 & \times 3-1 / 2 & \text { FIRE EXTINGUISHER (WITH ARROW DOWN) } \\
10 & \times 7 & \text { NOTICE - EMERGENCY USE ONLY }\end{array}$ & $\begin{array}{l}\text { EA } \\
\text { EA } \\
\text { EA } \\
\text { EA } \\
\text { EA }\end{array}$ \\
\hline $4280-34849$ & $\begin{array}{l}\text { LABEL, TAPE, PRESSURE SENSITIVE, 1-3/4 X } 5 \text { IN. } \\
\text { PEEL OFF, LEGEND - RADIATION SYMBOL, } 500 \text { LABELS } \\
\text { PER PACKAGE }\end{array}$ & PG \\
\hline $4280-34844$ & $\begin{array}{l}\text { LABEL, TAPE, SELF-ADHERING, YELLOW, } 3 / 4 \text { IN. HIGH, } \\
1 \text { IN. WIDE X } 180 \text { FT LONG, MAGENTA IMPRINT } \\
\text { RADIOACTIVE WITH SYMBOL, LENGTH OF IMPRINT } \\
3-1 / 2 \text { IN. }\end{array}$ & RO \\
\hline $4280-69620$ & $\begin{array}{l}\text { LABEL, TELEPHONE, EMERGENCY PHONE NUMBER AND } \\
\text { LOCATION, } 2 \text { IN. WIDE } X 1-1 / 8 \text { IN. HIGH, } \\
250 \text { LABELS PER ROLL }\end{array}$ & RO \\
\hline $280-63326$ & $\begin{array}{l}\text { SIGN, ADMINISTRATIVE ESCORT, BARRICADE, /VISITOR } \\
\text { UNCLASSIFIED DISCUSSIONS ONLY/ } 18 \times 24 \text { IN. } \\
\text { W. PLASTIC LEGS, PORT-A-CADE SALES MODEL P- } 24\end{array}$ & EA \\
\hline
\end{tabular}

STANDARD COLOR - LETTERS IN RED ON WHITE AND BLACK BACKGROUND. LETTERS 1 IN. HIGH SIZE 5-1/4 $52-1 / 2$ LABEL, SAFETY, WASTE CONTINUATION, SELF-ADHESIVE AGAINST FADING, 1-COLOR, BLACK LETTERS ON WHITE

SITIVE, ARROWS SH CKGROUND, 81 ARROWS PER SHEET

\section{RO} THIS CHEMICAT TO "1 sin LEGEND H.C. HOOD SURVEY - SATISFACTORY AVERAGE FACE VELOCITY, BLACK ON WHITE BACKGROUND, 3 LABELS IN. LEGEND H.L. HOOD SURVEY - SATISFACTORY UNDER FOLLOWING CONDITIONS, BLACK ON WHITE BACKGROUND, 2 IN. LEGEND H.C. HOOD SURVEY - SATISFACTORY UNDER FOLLOWING CONDITIONS, BLACK ON YELLOW BACKGROUND, LABEL, SAFETY, RADIOACTIVE MATERIAL ISOTOPE, WITH Ro RADIATION SYMBOL ATOMIC PRODUCTS CORP. N.Y. LABEL, SAFETY, RADIOACTIVE MATERIAL, LEGEND CAUTION CONTAMINATION HAZARD - DO NOT TOUCH RADIOACTIVE MATERIAL - WITH RADIATION SYMBOL. A 


\begin{tabular}{|l|l|l|}
\hline Catalog No. & Description & Unit \\
\hline
\end{tabular}

SIGN, FOR HAZARD AREA I.D. SYSTEM, RIGID VINXL, LAB SAFETY SUPPLY

LEGEND

NUMBER

\begin{tabular}{|c|c|}
\hline $\begin{array}{l}4280-71378 \\
4280-71379 \\
4280-71380\end{array}$ & $\begin{array}{l}R A-596-33 \\
R A-596-43 \\
R A-596-53\end{array}$ \\
\hline $4280-34831$ & $\begin{array}{l}\text { SIGN, HAZARD CONTROL, } 10 \times 14 \text { IN. WHITE } \\
\text { PAPERBOARD, HAZARDS CONTROL CIRCULAR SYMBOL AND } \\
\text { LEGEND, HAZARDS CONTROL PRINTED IN BLACK ACROSS } \\
\text { TOP EDGE }\end{array}$ \\
\hline $4280-71351$ & $\begin{array}{l}\text { SIGN, HAZARDOUS MATERIALS CLASSIFICATION, } \\
\text { NFPA RATING, SELF-ADHESIVE VINYL, } 8 \times 11 \text { IN. } \\
\text { LAB SAFETY RA-7171 }\end{array}$ \\
\hline
\end{tabular}

SIGN, NOTICE TO SMOKERS

4280-62874 NO SMOKING PLEASE, $9 \times 5-1 / 16$ IN. PLASTIC IEGEND IN BLACK AND WHITE, 2 TAPE STRIPS ON BACK TO ADHERE TO WALI, UNERAMED ROUND CORNERS

4280-62218 THANK YOU FOR NOT SMOKING HERE

$4280-62219$

SMOKERS PLEASE SIT NEAR ROOK EXHAUST OR REFRAIN EROM SMOKING, ETC.

4280-65435 SIGN, REPOSITORY, FOR PLACEMEN'T IN REPOSITORY EA

HANDLES TO INDICATE OPEN OR CLOSED CONDITION

FIRST SIDE - RED BACKGROUND COLOR W/LEGEND (ÓPEN)

IN WHITE COLOR, REVERSE SIDE - WHIT B BACKGROUND

COLOR W/LEGEND' (CLOSED) IN RED LETTERING

SIGN, SAFETX, CAUTION, $7 \times 10 \times 0.080$ IN. PLASTIC

MATERIAL STANDARD CAUTION FORMAT AND COLÓR - CAUTION - IN YELLOW LETTERS ON BLACK BACKGROUND, LEGEND IN BLACK LETTERS
ON YEILOW BACKGROUND

IEGEND

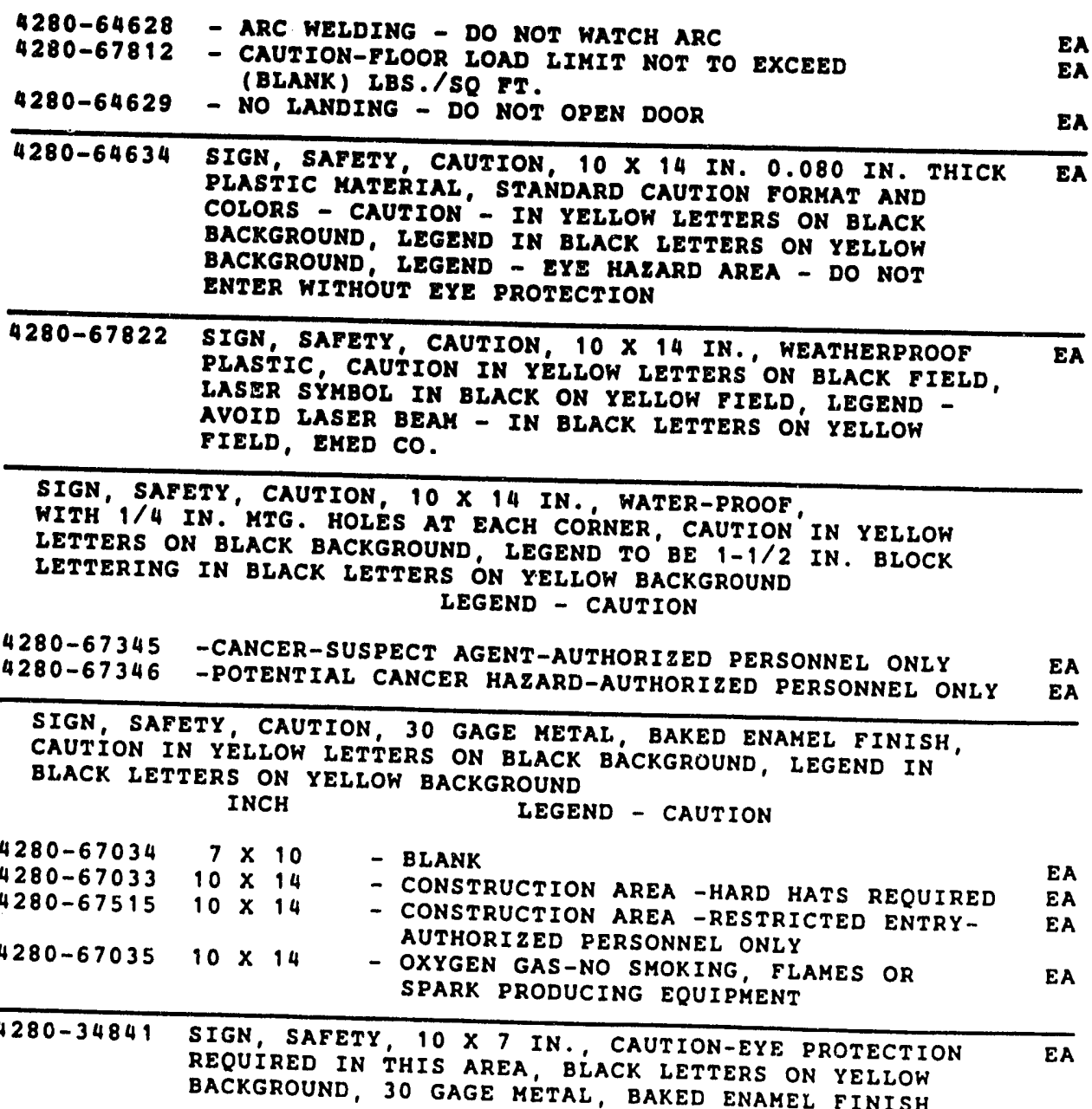
COLORS - CAUTION - IN YELIOW LETTERS ON BLACK BACKGROUND, LEGEND IN BLACK LETTERS ON YELLOW BACKGROUND, LEGEND - EYE HAEARD AREA - DO NOT

IASER SYMBOT IN BIACK ON YEILW FIELD ILEK FIELD AVOID LASER BEAM - IN BLACK LETTERS ON YEILOW FIELD, EMED CO. in in 
4280-70363 SIGN, SAFETY, DANGER, 7 IN. $X 5$ IN., SELF

ADHESIVE BACK, LEGEND: DANGER - CONF́INED SPACE -

ATMOSPHERE MAY BE HARMFUL TO LIFE - DO NOT ENTER

WITHOUT CONTACTING THE HAZARDS CONTROL SAFETY

TEAH AT. DANGER IN WHITE ON RED OVAL

BACKGROUND FRAMED IN BLACK, LEGEND LETTERS IN

BLACK ON WHITE BACKGROUND

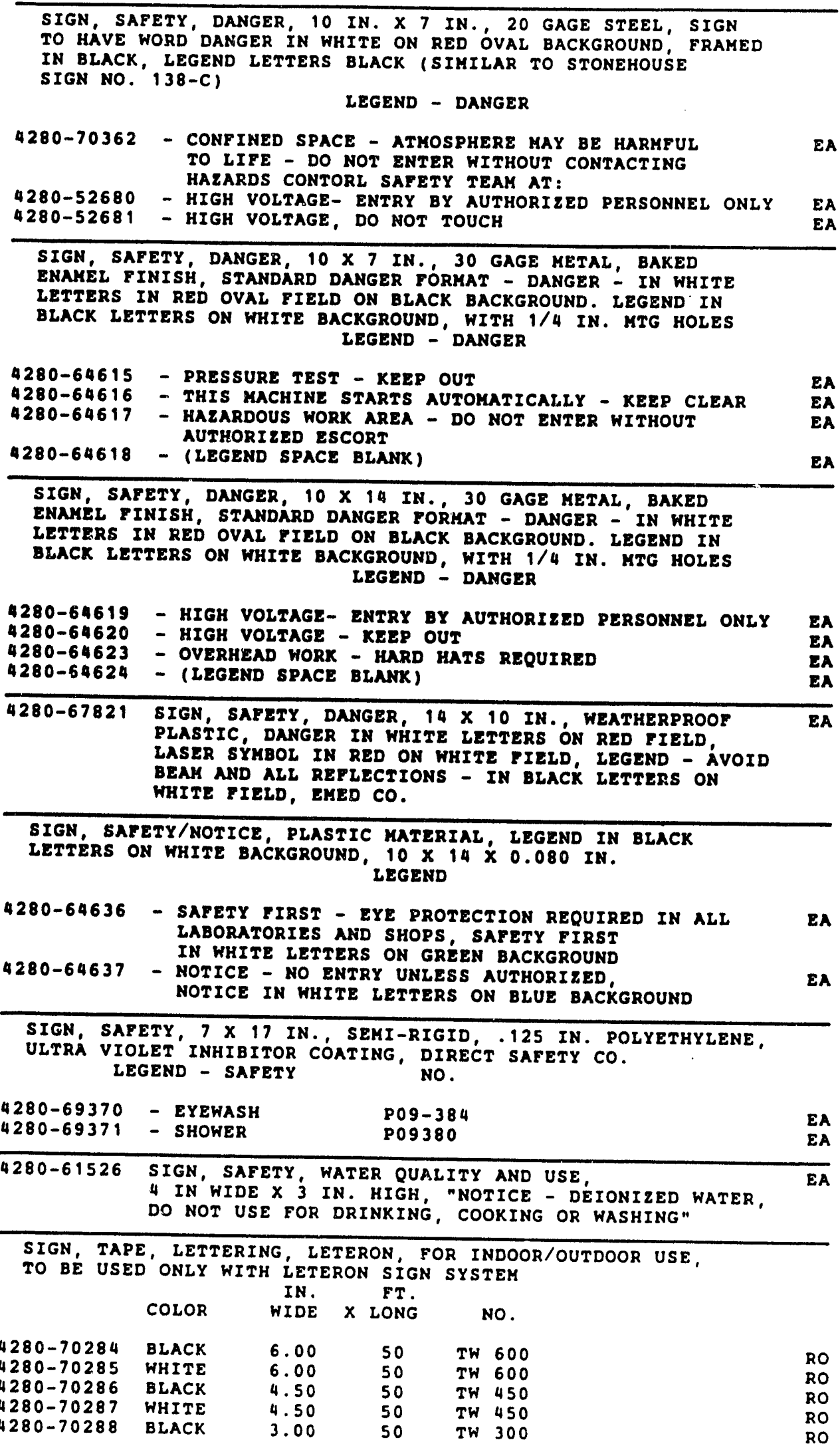


SIGN, TARE, LETTERING, LETERON, FOR INDOOR/OUTDOOR USE, TO BE USED ONLY WITH LETERON SIGN SYSTEM

$$
\text { COLOR WIDE } \times \text { LONG NO. }
$$

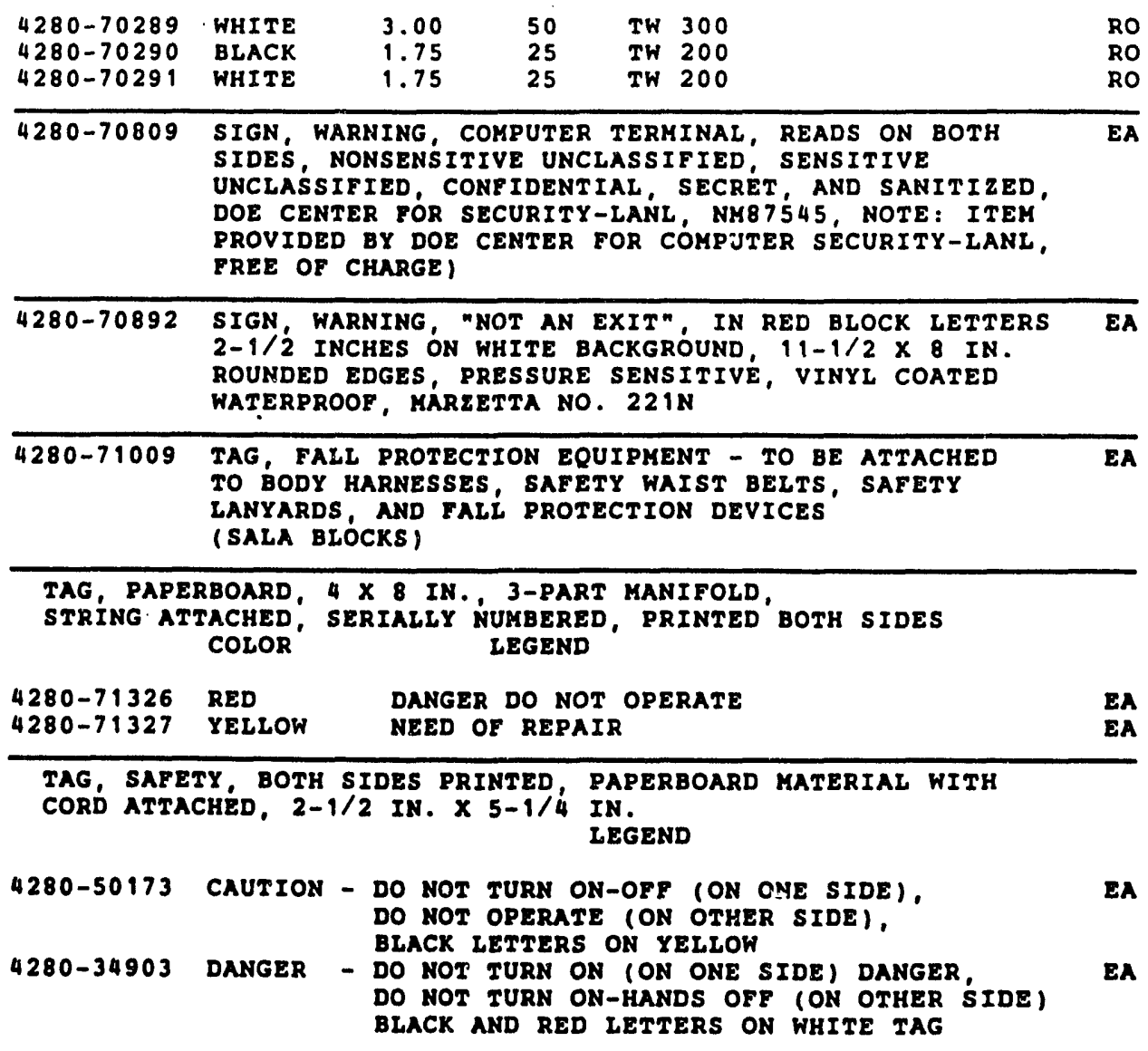

TAG, SAFETY, DANGER, FOR CIRCUIT BREAKERS, PAPERBOARD

MATERIAL WITH ROUND CENTER CUTOUT TO FIT ÓVER SWITCH

ACTUATOR, IMPRINTED BOTH SIDES WITH WHITE LETTERS ON RED

BACKGROUND, UPPER IEGEND-DANGER, BOTTOM IEGEND-DO NOT

OPERATE MEN WORKING

INCH

\begin{tabular}{llll}
$4280-41784$ & $1-1 / 2 \times 1-3 / 4$ & \\
$4280-41785$ & $2-3 / 8 \times 3-3 / 8$ & EA \\
\hline E280-34845 & $\begin{array}{l}\text { TAPE, HAZARD STRIPE, 1-1/2 IN. WIDE WITH } \\
\text { ALTERNATING 2 IN, WIDE BLACK AND YELIOW DIAGONAL }\end{array}$ & RO \\
STRIPES, REFLECTIVE BACKING, WESTLINE STOCK \\
HZ-102-C, 5 YDS PER ROLL
\end{tabular}




\section{Appendix 8}

\section{Chemical Storage Guidelines}




\section{Appendix 8 \\ Chemical Storage Guidelines}

\section{Storage Classes}

Storage of reactive chemicals by class (rather than alphabetically) ensures that individual chemicals receive the proper storage measures warranted by their reactivity. Incompatibilities between classes can be anticipated and protected against. Alphabetizing within a group, then, is acceptable. An added benefit to this type of storage is that knowledge of a chemical's reactivity is respected inside the storeroom and out. Once one recognizes a chemical's reactive class in the storeroom, the carry-over of this information to everyday laboratory exposure increases one's safety awareness.

\section{Acids}

Segregate acids from active metals such as sodium, potassium, magnesium, etc.

Segregate oxidizing acids from organic acids, flammable and combustible materials. Segregate acids from chemicals which could generate toxic or flammable gases upon contact, such as sodium cyanide, iron sulfide, calcium carbide, etc.

Segregate acids from bases.

\section{Bases}

Segregate bases from acids, metals, explosives, organic peroxides and easily ignitable materials.

\section{Solvents (Flammable and Halogenated Solvents)}

Store in approved safety cans or cabinets.

Segregate from oxidizing acids and oxidizers.

Keep away from any source of ignition: heat, sparks, or open flames.

\section{Oxidizers}

Store in a cool, dry place.

Keep away from combustible and flammable materials.

Keep away from reducing agents such as zinc, alkali metals, and formic acid. 


\section{Cyanides}

Segregate from acids and oxidizers.

\section{Water Reactive Chemicals}

Store in a cool, dry place away from any water source.

Have a Class $D$ fire extinguisher available in case of fire.

\section{Pyrophoric Substance}

(Materials which will react with the air to ignite when exposed, e.g., white phosphorus.)

Store in a cool, dry place making provisions for an airtight seal.

\section{Light Sensitive Chemicals}

Store in amber bottles in a cool, dry, dark place.

\section{Peroxidizable Chemicals}

Store in airtight containers in a dark, cool, and dry place.

Label containers with receiving, opening, and disposal dates.

Periodically test for the presence of peroxides.

\section{Toxic Chemicals}

Store according to the nature of the chemical, using appropriate security where necessary.

(From "Safe Chemical Storage: A Pound of Prevention is Worth a Ton of Trouble" by David Pipitone and Donald Hedberg, Journal of Chemical Education, Volume 59, Number 5, May 1982 and "Fire Protection Guide on Hazardous Materials," NFPA, 1978.) 


\section{Chemical Incompatibilities}

\section{Know the properties of the chemicals you use.}

The chemical incompatibilities discussed on the following pages are by no means exhaustive. As result, it is crucial for laboratory personnel to thoroughly research the properties of the chemicals they are using. Material Safety Data Sheets (MSDS's) all have sections on chemical incompatibility. While the quality of (MSDS's) varies from one manufacturer to another, they should serve as a primary resource for information or avoiding contact with incompatible compounds. A more detailed reference is the Handbook of Reactive Chemical Hazards, which can be ordered through Bretherick's Publishers.

\section{Avoid mixing incompatible waste materials.}

A number of serious laboratory accidents, have occurred when people have poured incompatible waste materials into hazardous waste containers. Use separate waste containers for each type of waste. Consult Appendix Six for more information on waste disposal.

\section{Store incompatible chemicals separately.}

Common storage problems in laboratories which could lead to mixing of incompatible chemicals. The most serious of these is the storage of acids (especially oxidizing acids) with flammable solvents. Contact of a concentrated oxidizing acid with a flammable solvent would likely result in a fire or an explosion. This is not an unlikely scenario in the event of an earthquake. Storage of chemicals in alphabetical order on shelves often results in incompatible chemicals being stored together. For example, alphabetical arrangement could result in hydrogen peroxide (a strong oxidizer) being stored next to hydrazine (a very strong reducer). 


\section{Examples of Incompatible Chemicals}

Chemical

Acetic Acid

Acetylene

Acetone

Alkali and alkaline earth metals (such as powdered aluminum or magnesium, calcium, lithium, sodium, potassium)

Ammonia (anhydrous)

Ammonium nitrate

Aniline

Arsenical materials

Azides
Is Incompatible With

Chromic acid, nitric acid, hydroxyl compounds, ethylene glycol, perchloric acid, peroxides, permanganates

Chlorine, bromine, copper, fluorine, silver, mercury

Concentrated nitric and sulfuric acid

Water, carbon tetrachloride or other chlorinated hydrocarbons, carbon dioxide, halogens

Mercury (in manometers, for example), chlorine, calcium hypochlorite, iodine, bromine, hydrofluoric acid (anhydrous)

Acids, powdered metals, flammable liquids, chlorates, nitrates, sulfur, finely divided organic combustible materials

Nitric acid, hydrogen peroxide

Any reducing agent

Acids

App. 8 - 4 


\section{Chemical}

Bromine

Calcium oxide

Carbon (activated)

Carbon tetrachloride

Chlorates

Chromic acid \& chromium trioxide

Chlorine

Chlorine dioxide

Copper

Cumene hydroperoxide

Cyanides

Flammable liquids

\section{Is Incompatible With}

See chlorine

Water

Calcium hypochlorite, all oxidizing agents

Sodium

Ammonium salts, acids, powdered metails, sulfur, finely divided organic or combustible materials

Acetic acid, naphthalene, camphor, glycerol, alcohol, flammable liquids in general

Ammonia, acetylene, butadiene, butane, methane, propane (or other petroleum gases), hydrogen, sodium carbide, benzene, finely divided metals, turpentine

Ammonia, methane, phosphine, hydrogen sulfide Acetylene, hydrogen peroxide

Acids (organic or inorganic)

Acids

Ammonium nitrate, chromic acid, hydrogen peroxide, nitric acid, sodium peroxide, halogens 
Hydrocarbons (such as butane, Fluorine, chlorine, bromine, chromic acid, sodium propane, benzene) peroxide

Hydrocyanic acid Nitric acid, alkali

Hydrofluoric acid (anhydrous) Ammonia (aqueous or anhydrous)

Hydrogen peroxide

Copper, chromium, iron, most metals or their salts, alcohols, acetone, organic materials, aniline, nitromethane, combustible materials

Hydrogen sulfide

Fuming nitric acid, oxidizing gases

Hypochlorites

Acids, activated carbon

Iodine

Acetylene, ammonia (aqueous or anhydrous), hydrogen

Mercury

Acetylene, fulminic acid, ammonia

Nitrates

Sulfuric acid

Nitric acid (concentrated)

Acetic acid, aniline, chromic acid, hydrocyanic acid, hydrogen sulfide, flammable liquids, flammable gases, copper, brass, any heavy metals

Nitrates

Acids

Nitroparaffins

Inorganic gases, amines

Oxalic acid

Silver, mercury 
Chemical

Oxygen

Perchloric acid

Peroxides, organic

Phosphorus (white)

Potassium

Potassium chlorate

Potassium perchlorate (see also chlorates)

Potassium permanganate

Selenides

Silver

Sodium

Sodium nitrite

\section{Is Incompatible With}

Oils, grease, hydrogen: flammable liquids, solids or gases

Acetic anhydride, bismuth and its alloys, alcohol, paper, wood, grease, oils

Acids (organic or mineral), avoid friction, store cold

Air, oxygen, alkalies, reducing agents

Carbon tetrachloride, carbon dioxide, water

Sulfuric and other acids

Sulfuric and other acids

Glycerol, ethylene glycol, benzaldehyde, sulfuric acid

Reducing agents

Acetylene, oxalic acid, tartartic acid, ammonium compounds, fulminic acid

Carbon tetrachloride, carbon dioxide, water Ammonium nitrate and other ammonium salts 
Chemical

Sodium peroxide
Ethyl or methyl alcohol, glacial acetic acid, acetic anhydride, benzaldehyde, carbon disulfide, glycerin, ethylene glycol, ethyl acetate, methyl acetate, furfural

Acids

Sulfides

Sulfuric acid

Potassium chlorate, potassium perchlorate, potassium permanganate (similar compounds of light metals, such as sodium, lithium)
Reducing agents

From: "Safety in Academic Chemistry Laboratories," American Chemical Society 


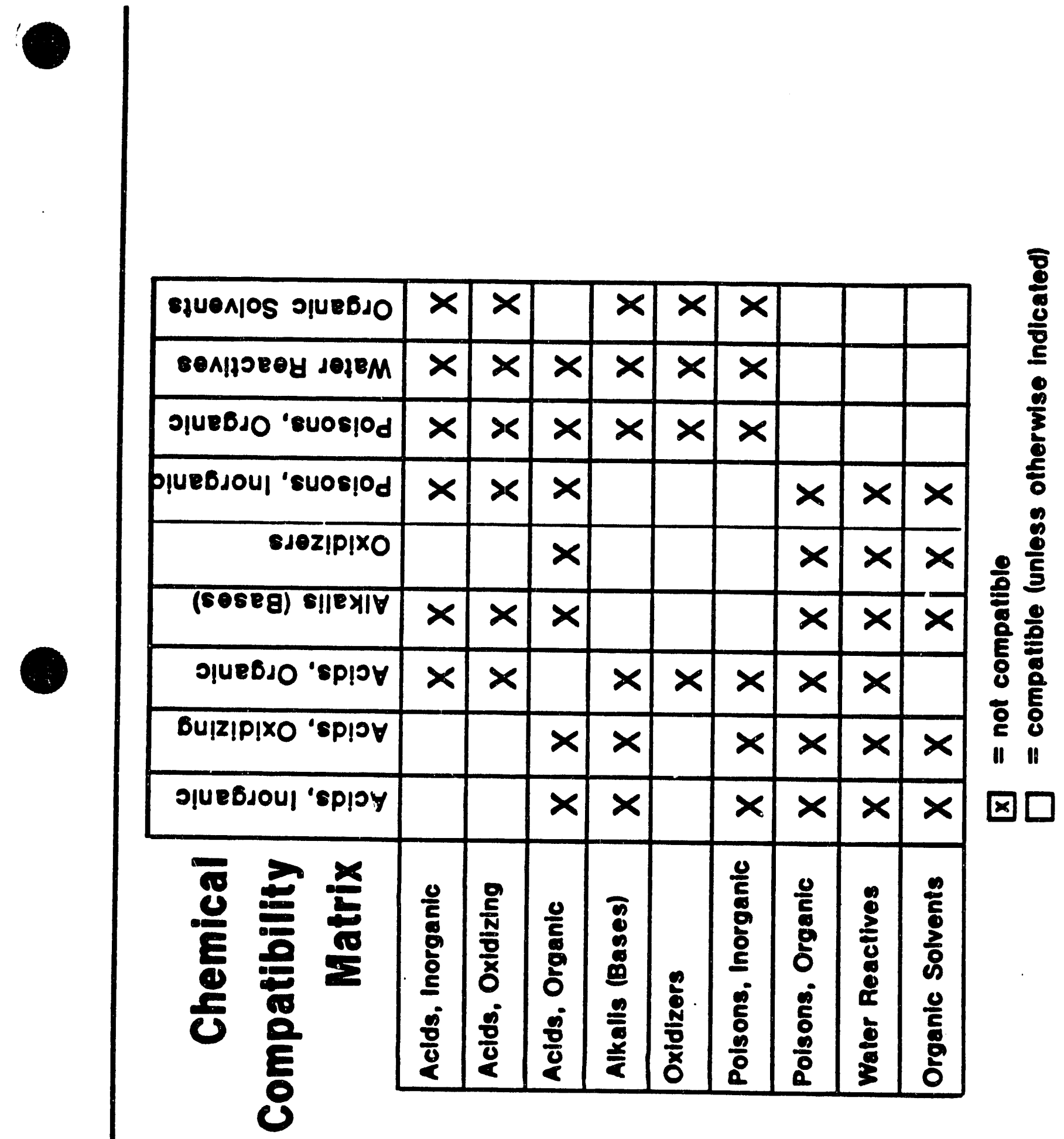


Appendix 9

Select Carcinogens 


\section{Appendix 9 \\ Select Carcinogens}

(Pursuant to the OSHA Laboratory Standard, 29 CFR 1910.1450)

A. OSHA Regulated Carcinogens (29 CFR 1910.1000, Subpart Z)

2-acetylaminofluorene

acrylonitrile

4-aminodiphenyl

inorganic arsenic

asbestos

benzene

benzidine and its salts

1,2-dibromo-3-chloropropane

3,3 '-dichlorobenzidine and its salts

4-dimethylaminoazobenzene

ethylene oxide

formaldehyde

alpha-naphthylamine

beta-naphthylamine

4-nitrobiphenyl

$\mathrm{N}$-nitrosodimethylamine

beta-propiolactone

bis-chloromethyl ether

methyl chloromethyl ether

ethyleneimine

vinyl chloride (and polyvinyl chloride)

B. I.A.R.C. Identified Carcinogens ${ }^{a}$

Group 1 "Agents that are Carcinogenic to Humans"

aflatoxins production

4-aminobiphenyl

analgesic mixtures containing phenacetin

arsenic and arsenic compounds

asbestos

auramine, manufacture of

azathioprine

benzene

benzidine

betel quid with tobacco

N,N-bis(2-chloroethyl)-2-naphthylamine (Chlornaphazine) 
bis(chloromethyl) ehter and chloromethyl ether (technical-grade)

boot and shoe manufacture and repair

1,4-butanediol

dimethanesulphonate (Myleran)

chlorambucil

1-(2-chloroethyl)-3(4-methylcyclohexyl)-1-nitrosourea (methyl-CCNU)

chromium compounds, hexavalent

coal gasification

coal-tar pitches

coal-tars

coke production

cyclophosphamide

ciethylstilboestrol

drionite

furniture and cabinet making

haematite mining, underground, with exposure to radon

iron and steel founding

isopropyl alcohol manufacture, strong-acid process

magenta, manufacture of

melphalan

8-methoxypsoralen (methoxsalen) plus ultraviolet radiation

mineral oils, untreated and mildly-treated

MOPP (combined therapy with nitrogen mustard, vincristine, procarbazine and prednisone) and other combined chemotherapy including alkylating agents.

mustard gas (sulphur mustard)

2-naphthylamine

nickel and nickel compounds

oestrogen replacement therapy

oestrogens, nonsteroidal

oestrogens, steroidal

oral contraceptives, combined

oral contraceptives, sequential

the rubber industry

shale-oils

soots

talc containing asbestiform fibers

tobacco products, smokeless

tobacco smoke

treosulphan

vinyl chloride

Group 2A:"Agents that are Probably Carcinogenic to Humans"

acrylonitrile

adriamycin

androgenic (anabolic) steroids

App. 9 - 2 


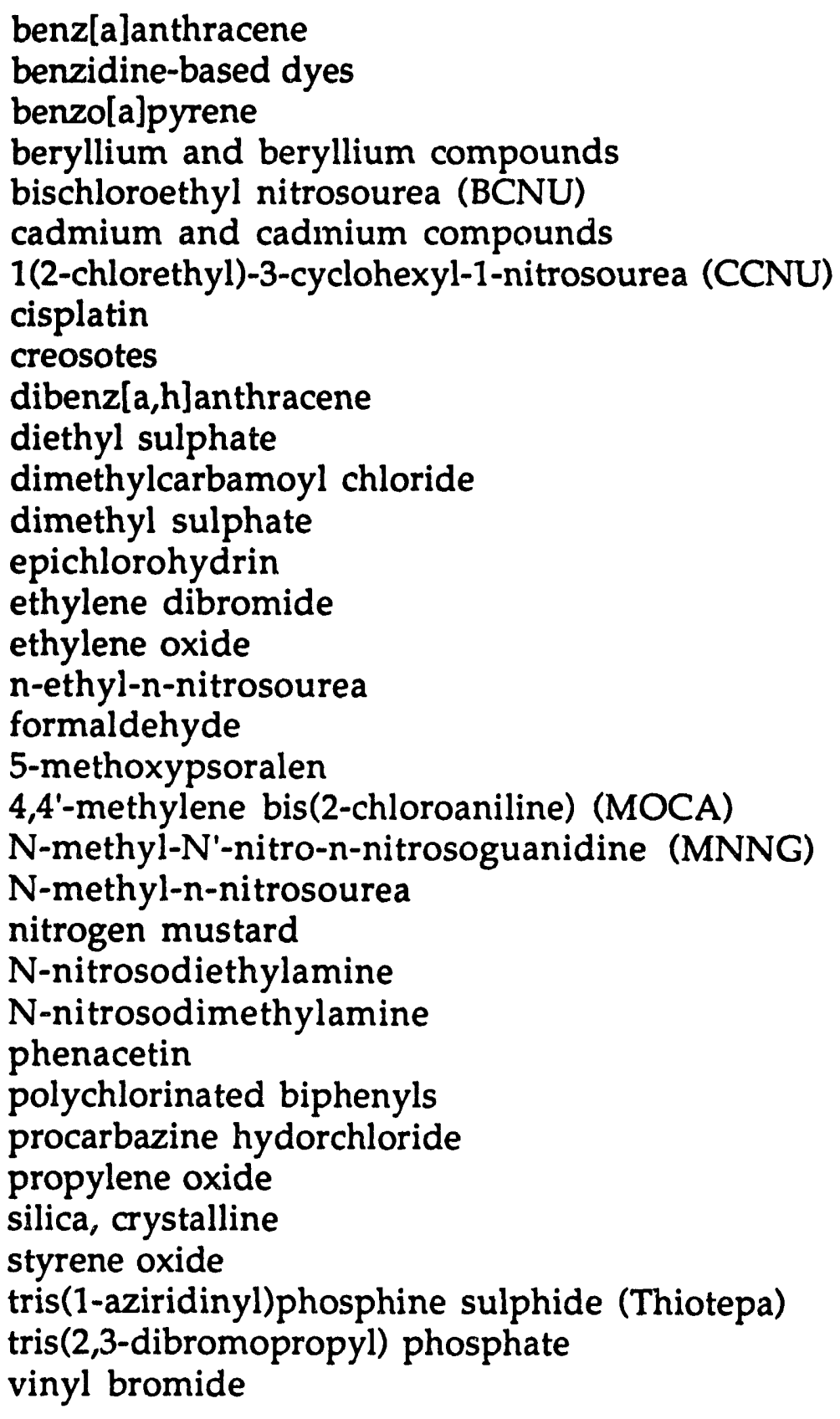

Group 2B."Agents that are Possibly Carcinogenic to Humans"

a-alpha, C (2-amino-9H-pyrido[2,3-b]indole)

acetaldehyde

acetamide

acrylamide AF-2 [2 22-furyl\}-3-(5-nitro-2-furyl) acrylamide]

para-aminoazobenzene

ortho-aminoazotoluene

2-âtino-5-(5-nitro-2-furyl)-1,3,4-thiadiazole

amitrole

ortho-anisidine 
aramite

auramine, technical-grade

azaserine

benzo[b]fluoranthene

benzo(j)fluoranthene

benzo[k]fluoranthene

benzyl violet $4 B$

bitumens, extracts of steam-refined and air-refined

bleomycins

bracken fern

1,3-butadiene

butylated hydroxyanisole (BHA)

beta-butyrolactone

carbon-black extracts

carbon tetrachloride

carpentry and joinery

carrageenan, degraded

chloramphenicol

chlordecone (Kepone)

alpha-chlorinated toluenes

chloroform

chlorophenols

chlorophenoxy herbicides

4-chloro-ortho-phenylenediamine

para-chloro-ortho-toluidine

citrus red no. 2

para-cresidine

cycasin

dacarbazine

daunomycin

DDT

$\mathrm{n}, \mathrm{n}$-diacetylbenzidine

2,4-diaminoanisole

4,4'-diaminodiphenyl ether

2,4-diaminotoluene

dibenz[a,h]acridine

dibenz[a,j]acridine

7H-dibenzo[c,g]carbazole

dibenzo[a,e]pyrene

dibenzo[a,h]pyrene

dibenzo[a,l]pyrene

1,2-dibromo-3-chloropropane

para-dichlorobenzene

3,3'-dichlorobenzidine

3,3'-dichloro-4,4'-diaminodiphenyl ether

1,2-dichloroethane 
dichloromethane

1,3-dichloropropene (technical-grade)

diepoxybutane

di(2-ethylhexyl)phthalate

1,2-diethyldrazine

diglycidyl resorcinol ether

dihydrosafrole

3,3'-dimethoxybenzidine (ortho-dianisidine)

para-dimethylaminoazobenzene trans-2[(dimethylamino)methylimino]-5[2(5-nitro-

2-furyl)vinyl]-1,3,4-oxadiazole

3,3'-dimethylbenzidine (ortho-tolidine)

1,1-dimethylhydrazine

1,2-dimethylhydrazine

1,4-dioxane

ethyl acrylate

ethylene thiourea

ethyl methanesulphonate 2'-2-Formylhydrazino(-4'-5nitro-2-furyl) thiazole

glu-P-1 (2-amino-6-methyldipyrido[1,2-a:3', 2'-d]imidazole)

glu-P-2 (2-aminodipyrido[1,2-a:3', 2'-d]imidazole)

glycidaldehyde

griseofulvin

hexachlorobenzene

hexachlorocyclohexanes

hexamethylphosphoramide

hydrazine

indeno[1,2,3-cd]pyrene

IQ (2-amino-3-methylimidazo[4,5-f]quinoline)

iron-dextran complex

lasiocarpine

lead and lead compounds, inorganic

mea-alpha-c (2-amino-3-methyl-9H-pyrido[2,3-b]indole)

medroxyprogesterone acetate

merphalan

2-methylaziridine

methylazoxymethanol and its acetate

5-methylchrysene

4,4'-methylene bis(2-methylaniline)

4,4'-methylenedianiline

methyl methanesulphonate

2-methyl-1-nitroanthraquinone (uncertain purity)

n-methyl-n-nitrosourethane

methylthiouracil

metronidazole

mirex

mitomycin $\mathrm{C}$

monocrotaline 
5-(morpholinomethyl)-3-[(5-nitrofurfurylidene)amino]-2-oxazolidinone Nafenopin

Niridazole

5-nitroacenaphthene

nitrofen (technical-grade)

1- [(5-nitrofurfurylidene)amino]-2-imidazolidinone

$\mathrm{N}-\mathrm{N}$ - [4(5-nitro-2-furyl)-2-thiazolyl]acetamide

nitrogen mustard $\mathrm{N}$-oxide

2-nitropropane

$\mathrm{N}$-nitrosodi-n-butylamine

$\mathrm{N}$-nitrosodiethanolamine

$\mathrm{N}$-nitrosodi-n-propylamine

3-(N-nitrosomethylamino)propionitrile

4-(N-nitrosomethylamino)-1(3-pyridyl)-1-butanone (NNK)

$\mathrm{N}$-nitrosomethylethylamine

$\mathrm{N}$-nitrosomethylvinylamine

$\mathrm{N}$-nitrosomorpholine

$\mathrm{N}$-nitrosonornicotire

$\mathrm{N}$-nitrosopiperidine

$\mathrm{N}$-nitrosopyrrolidine

$\mathrm{N}$-nitrososarcosine

oil orange SS

panfuran S (containing dihydroxymethylfuratrizine)

phenazopyridine hydrochloride

phenobarbital

phenoxybenzamine hydrochloride

phenytoin

polybrominated biphenyls

ponceau $\mathrm{MX}$

ponceau $3 R$

potassium bromate

progestins

1,3-propane sultone

b-propiolactone

propylthiouracil

saccharin

safrole

sodium ortho-phenylphenate

sterigmatocystin

streptozotocin

styrene

sulfallate

2,3,7,8-tetrachlorodibenzo-para-dioxin (TCDD)

tetrachloroethylene

thioacetamide

4,4'thiodianiline

App. 9 - 6 
thiourea

toluene diisocyanates

ortho-toluidine

toxaphene (polychlorinated camphenes)

trp-P-1 (3-Amino-1,4-dimethyl-5H-pyrido[4,3-b]indole)

trp-P-2 (3-Amino-1-methyl-5H-pyrido[4,3-b]indole)

trypan blue

uracil mustard

urethane

C. NTP Identified Carcinogens b

Group 1. "Substances Known to be Carcinogens"

4-aminobiphenyl

analgesic mixtures containing phenacetin

arsenic and certain arsenic compounds

asbestos

azathioprine

benzene

benzidine

bis(chloromethyl)ether and technical grade chloromethyl methyl ether

1,4-butanediol dimethylsulfonate (Myleran)

chlorambucil

chromium and certain chromium compounds

conjugated estrogens

cyclophosphamide

diethylstilbestrol

melphalan

methoxsalen with ultra-violet A therapy (PUVA)

mustard gas

2-naphthylamine

thorium dioxide

vinyl chloride

Group 2. "Substances Reasonably Anticipated to be Carcinogens"

2-acetylaminofluorene

acrylonitrile

adriamycin

aflatoxins

2-aminoanthraquinone

o-a minoazotoluene

1-amino-2-methylanthraquinone

amitrole

o-anisidine hydrochloride 
benzotrichloride

beryllium and certain beryllium compounds

bischloroethyl nitrosourea

1,3-butadiene

cadmium and certain cadmium compounds

carbon tetrachloride

chlorendic Acid

chlorinated paraffins (C12 60\% chlorine)

1-(2chloroethyl)-3-cyclohexyl-1-nitrosourea (CCNU)

chloroform

3-chloro-2-methylpropene

4-chloro-o-phenylenediamine

c.1. basic red 9 monohydrochloride

p-cresidine

cupferron

dacarbazine

DDT

2,4-diaminoanisole sulfate

2,4-diaminotoluene

1,2-dibromo-3-chloropropane

1,2-dibromoethane (EDB)

1,4-dichlorobenzene

3,3'-dichlorobenzidine and 3,3'-dichlorobenzidinedihydrochloride

1,2-dichloroethane

dichloromethane (methylene chloride)

1,3-dichloropropene (technical grade)

diepoxybutane

di(2-ethylhexyl)phthalate

diethy sulfate

dig1 ycidyl resorcinol ether

3,3'-dimethoxybenzidine

4-dimethylaminozobenzene

3, 3'-dimethyl benzidine

dimethylcarbimoyl chloride

1,1-dimethylhydrazine

dimethyl sulfate

dimethylvinyl chloride

1, 4-dioxane

direct black 38

direct blue 6

epichlorochydrin

estrogens (not nonjugated): estradiol-17(beta)

estrogens (not nonjugated): estrone

estrogens (not nonjugated): ethinylestradiol

estrogens (not nonjugated): mestranol

ethyl acrylate 
ethylene oxide

ethylene thiourea

formaldehyde (gas)

hexachlorobenzene

hexamethylphosphoramide

hydrazine and hydrazine sulfate

hydrazobenzene

iron dextran complex

kepone (chlordecone)

lead acetate and lead phosphate

lindane and other hexachlorocyclohexane isomers

2-methylaziridine (Propyleneimine)

4,4'-methylenebis (2-chloroaniline) (MBOCA)

4,4'-methylenebis( $n, n$-dimethyl) benzenamine

4,4'-methylenedianiline and its dihydrochloride

metronidazole

Michler's ketone

Mirex

nickel and certain nickel compounds

nitrilotriacetic acid

5-nitro-o-anisidine

nitrofen

nitrogen mustard hydrochloride

2-nitropropane

$\mathrm{N}$-nitrosodi-N-butylamine

$\mathrm{N}$-nitrosodiethanolamine

$\mathrm{N}$-nitrosodiethylamine

$\mathrm{N}$-nitrosodimethylamine

p-nitrosodiphenylamine

n-nitrosodi-n-propylamine

$\mathrm{N}$-nitroso-N-ethylurea

$\mathrm{N}$-nitroso-N-methylurea

$\mathrm{N}$-nitrosomethylvinylamine

$\mathrm{N}$-nitrosomorpholine

$\mathrm{N}$-nitrosonornicotine

$\mathrm{N}$-nitrosopiperidine

$\mathrm{N}$-nitrosopyrrolidine

$\mathrm{N}$-nitrososarcosine

norethisterone

4,4'-oxydianiline

oxymetholone

phenacetin

phenazopyridine hydrochloride

phenoxybenzamine hydrochloride

phenytoin

polybrominated biphenyls

App. 9 - 9 
polychlorinated biphenyls

polycyclic aromatic hydrocarbons, 15 listings:

benz(a)anthracene

benzo(b)fluoranthene

benzo(j)fluoranthene

benzo(k)fluoranthene

benzo(a)pyrene

dibenz $(a, h)$ acridine

dibenz $(a, j)$ acridine

dibenz $(a, h)$ anthracene

Th-dibenzo(c,g)carbazole

dibenzo(a,e)pyrene

dibenzo $(a, h)$ pyrene

dibenzo $(a, i)$ pyrene

dibenzo $(a, 1)$ pyrene

indeno(1,2,3-cd)pyrene

5-miethylchrysene

procarbazine hydrochloride

progesterone

1,3-propane sultone

(beta)-propiolactone

propylene oxide

propylthiouracil

reserpine

saccharin

safrole

selenium sulfide

streptozotocin

sulfallate

2,3,7,8-tetrachlorodibenzo-p-dioxin (TCDD)

tetrachloroethylene (perchloroethylene)

thioacetamide

thiourea

toluene diisocyanate

o-toluidine and o-toluidine hydrochloride

toxaphene

2,4,6-trichlorophenol

tris (1-aziridinyl)phosphine sulfide

tris (2,3-dibromopropyl)phosphate

urethane

a IARC (International Agency for Research on Cancer) lists from 1987 monographs.

b NTP (National Toxicology Program) lists from the "Fourth Annual Report on Carcinogens," 1985. 
Appendix 10

ACGIH Carcinogens 


\title{
Appendix 10 \\ ACGIH Carcinogens*
}

\author{
A1 Carcinogens \\ 4-aminodiphenyl \\ asbestos \\ - amosite \\ - chrysotile \\ - crocidolite \\ - other forms \\ benzidine \\ bis(chloromethyl)ether \\ chromite ore processing \\ chromium (VI) compounds, certain water insoluble \\ coal tar pitch volatiles, benzene solubles \\ nickel sulfide roasting \\ 4-nitrodiphenyl \\ vinyl chloride \\ zinc chromates
}

\section{A2 Carcinogens}

acrylamide

acrylonitrile

antimony trioxide production

arsenic trioxide production

benzene

benzo(a)pyrene

beryllium

1,3-butadiene

carbon tetrachloride

chloroform

chloromethyl methyl ether

chrysene

3,3'-bichlorobenzidine

bimethyl carbamoyl chloride

1,1-dimethylhydrazine

dimethyl sulfate

ethyl acrylate

ethylene dibromide

ethylene oxide 
ACGIH A2 Carcinogens, continued

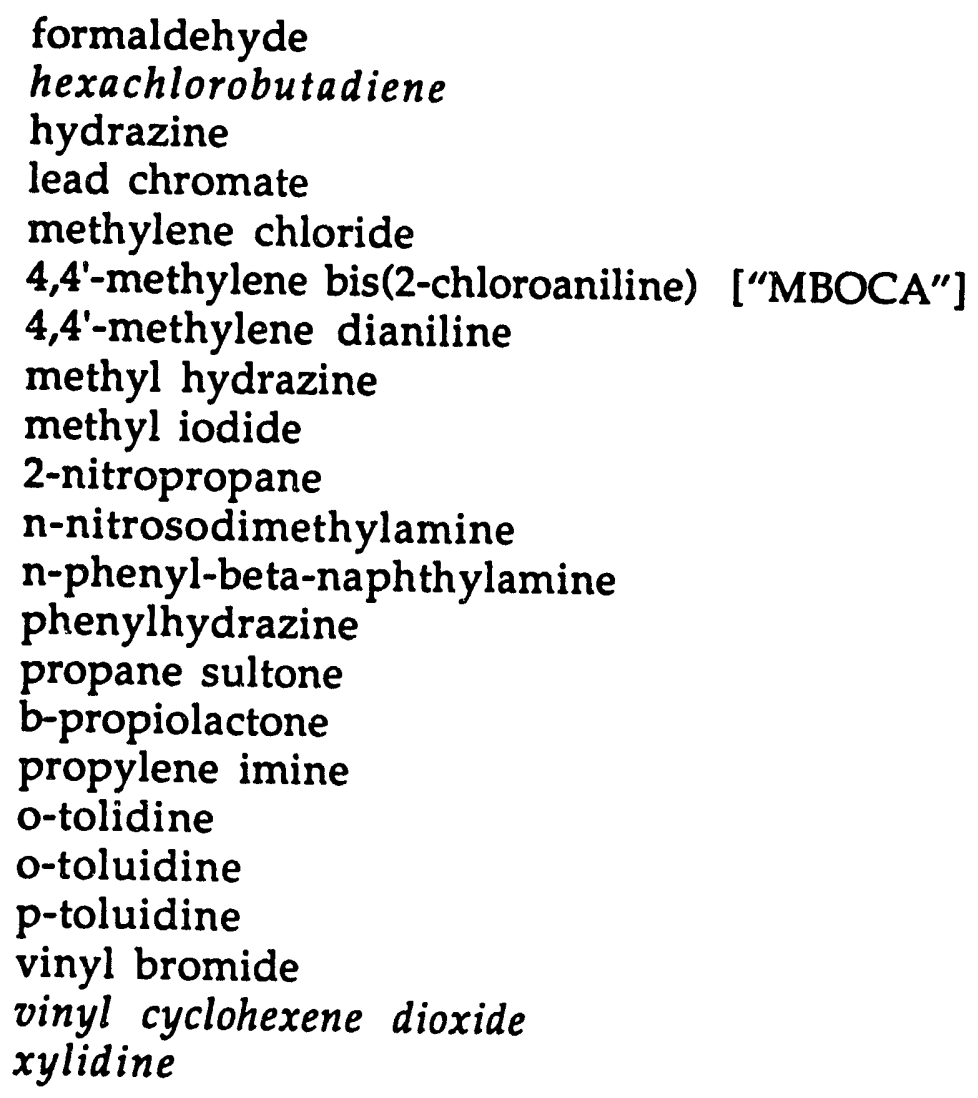

${ }^{*} \mathrm{ACGIH}$ is the American Conference of Governmenal Industrial Hygienists.

"A1" designates a confirmed human carcinogen: a substance, or substances associated with industrial processes, recognized to have carcinogenic
potential.

"A2" designates a suspected human carcinogen: a substance or substances associated with industrial processes, which are suspect of inducing cancer, based on either limited epidemiological evidence or demonstration of carcinogenesis in one or more animal species by appropriate methods.

Note: Italicized entries (four of the listings) are not included in the current OSHA Select Carcinogen List (Appendix 9). 
Appendix 11

Composite Working Select Carcinogen List 
ACETAMIDE

2-ACETYLAMIMOFLUORENE

ACRYLAHIDE

ACRYLONITRILE

ADRIAMYCIN

AF-2 (2(2-FURYL)-3-(5-NITRO-2-FURYL) ACRYLAMIDE

AFLATOXINS

A-ALPHA, C (2-AMINO-9H-PYRIDO[2,3-8] INDOLE)

1-AMINO-2-METHYLANTHRAOUINOHE

2-AMINO-5-(5-NITRO-FURYL)-1,3,4-THIADIAZOLE

2-AMINOAHTHRAQUINONE

AMINOAZOBENZENE

O-AMINOAZOTOLUENE

AMINOAZOTOLUENE

4-AMINOBIPHENYL

AMITROLE

AMDROGENIC STEROIDS

ANISIDINE

ANISIDINE HYOROCHLORIOE

ARAMITE

ARSENIC

ARSENIC ACIO

ARSENIC ACID

ARSENIC ACID, CALCIUM SALT

ARSENIC ACID, CALCIUM SALT

ARSENIC ACID, DIAMMONIUM SALT

ARSENIC ACID, DISODIUM SALT

ARSENIC ACID, DISCOIUM SALT, HEPTAHYORATE

ARSENIC ACID, HEMIHYORATE

ARSENIC ACID, LEAD SALT

ARSENIC ACID, LEAD(2+) SALT(1:1)

ARSENIC ACID, LEAD(2+) SALT(2:3)

ARSENIC ACID, MAGNESIUM SALT

ARSENIC ACID, MONOPOTASSIUM SALT

ARSENIC ACID, MONOSOOIUM SALT

ARSENIC ACID, SODIUM SALT

ARSENIC ACID, TRICESIUM SALT

ARSENIC CHLORIDE

ARSENIC IOOIDE

ARSENIC PENTOXIDE

ARSENIC SULFIDE

ARSENIC SULFIDE

ARSENIC TRIOXIDE

ARSENIC (II) BROMIDE

ARSENIC(V) ACID, TRISOOIUM SALT, HEPTAHYDRATE

ARSENIOUS ACID, CALCIUM SALT

ARSENIOUS ACID, COPPER(II) SALT(1:1)

ARSENIOUS ACID, MONOSODIUM SALT

ARSENIOUS ACID, POTASSIUN SALT

ARSENIOUS ACID, SOOIUM SALT
W-FLUOREN-2YL ACETAMIDE

$602-87-9$

$60-35-5$

53-96-3

79-06-1

107-13-1

23214-92-8

3688-53-7

$1402-68-2$

AMINO-ALPHA-CARBOLINE

26148-68-5

$82-28-0$

$712-68-5$

$117-79-3$

para-aminoazobenzene; Phenylazoaniline

60-09-3

2-AMINO-5-AZOTOLUENE

$97-56-3$

ORTHO-AMINOAZOTOLUENE

97-56-3

4-AMINOBIPHENYL

92-67-1

AMINOTRIAZOLE

$61-82-5$

ANABOLIC STEROIDS

ORTHO-ANISIOINE

ORTHO-ANISIDINE HYDROCHLORIDE

90-04-0

2(P-TERT-BUTYLPHENOXY)-1-METHYLETHYL-2-CHLOROETHYL 140-57-8

$7440-38-2$

$7778-38-4$

10102-53-1

10103-62-5

$7778-44-1$

$7784-44-3$

7778-43-0

10048-95-0

$7774-41-6$

$7645-25-2$

$7784-40-9$

$3687-31-8$

10105-50-1

$7784-41-0$

101003-60-3

7631-89-2

6436-62-1

$7784-34-1$

$7784-45-4$

1303-28-2

$56320-22-0$

1303-33-9

1327-53-3

$7784-33-0$

64070-83-3

27152-57-4

10290-12-7

$7784-46-5$

10124-50-2

14080-36-9 
CHEMICAL

ARSENIOUS ACID, TRISILVER(1+) SALT

ARSEMIOUS ACID, ZINC SALT

ARSENIOUS TRIFLUORIDE

ARSONIC ACID, CALCIUN SALT (1:1)

ASBESTOS

AURAMIME

AZASERIME

AZATHIOPRINE

BENZ(A)ANTHRACENE

SYNOWYM

BENZENE

BENZIDINE

$7784-08-0$

$10326-24-6$

$7784-36-2$

52740-16-5

1332-21-4

492-80-8

$115-02-6$

446-86-6

56-55-3

$71-43-2$

$92-87-5$

BENZO(A)PYREME

50-32-8

BENZO(B)FLUORANTHENE

205-99-2

BENZO(d)FLUORANTHENE

205-82-3

205-82-3

BENZO(J)FLUORANTHENE

207-08-9

BENZOTRICHLORIDE

$98 \cdot 07 \cdot 7$

BENZYL VIOLET $4 B$

1694-09-3

BERYLLIU:

BERYLLIUM ALUMINIUM ALLOY

$7440-41-7$

BERYLLILM BIS(CARBANATO(2-))DIHYDROXYTRI-

12770-50-2

$66104-24-3$

BERYLLIUN CHLORIDE

BERYLLIUN FLUORIDE

$7787-47-5$

BERYLLIUM HYOROGEN PHOSPHATE

$7787-49-7$

BERYLLIUN HYDROXIDE

$13598-15-7$

BERYLLIUH OXIDE

$13327-32-7$

BERYLLIUM SULFATE $(1: 1)$

$1304-56-9$

BERYLLIUM SULFATE, TETRAHYDRATE

BIS(2-CHLOROETHYL)-2-MAPHTHAYLAMIME

CHLORNAPHAZINE

$13510-49 \cdot 1$

$7787-56-6$

BIS(CHLOROMETHYL) ETHER

DICHLOR.COIMETHYL ETHER

$494-03 \cdot 1$

BISCHLOROETHYL NITROSOUREA

BITUMENS, EXTRACTS OF STEAM-REFINED AND AIR-REFINE

BCNU

$542 \cdot 88 \cdot 1$

$154-93-8$

BLEOMYCIN

$11056-06-7$

BRACKEN FERM

1,3-BUTADIEME

PYRROL YLLENE

106-94-0

1,4-BUTANEDIOL DIMETHANESULFONATE

MYLERAIH

$55-98-1$

BUTYLATEO HYDROXYANISOLE

BETA-BUTYROLACTONE

BHA; SUSTANE; ANTHRACINE 12

25013-16-5

4-METHYL-2-OXYTANONE

3068-88-0

$569-61.9$

$7740-43-9$

CAOMIUN

CADMIUA CHLORIDE

CADMIUA OXIDE

CADMIUM SULFATE

CADMIUN SULFIDE

7H-CARBAZOLE

DIBENZO(C, G)CARBAZOLE

$10108-64-2$

1306-19-0

$10124-36-4$

$1306 \cdot 23-6$

$194-59-2$

56-23-5

CARBON-BLACK EXTRACTS

$305-03-3$

$56-75-7$

$115-28-6$

CHLORENDIC ACIO

$108171-26-2$ 
-CHLORO-2-METHYLPROPENE

4-CHLORO-0-PHENYLENEDIAMINE CHLORO-ORTHO-TOLUIDINE

1-(2-CHLOROETHYL)-3(4-METHYLCYCLO-HEXYL) - 1-NITROSO

1-(2-CHLOROETHYL)-3-CYCLOHEXYL-1-NITROSOUREA

CHLOROFORM

CHLOROMETHYL METHYL ETHER

CHLOROPHENOLS

CHLOROPHENOXY HERBICIDES

CHROMTE(1-) HYDROXYOCTAOXOOIZINCATED POTASSIUM

CHROAIC ACID, CALCILM SALT

CHROAIC ACIO, DISOOIUM SALT

CHROMIC ACID, LEAD(2+) SALT

CHROMIC ACID, POTASSIUN SALT

CHROAIC ACID, STROWTIUN SALT

CHROMIC ACID, ZINC HYDROXIDE HYDRATE

CHROMIC ACID, ZINC SALT

CHROMIUM CHLORIDE,

CHROMIUM OICHLOROOIOXO

CHROMIUM OXIDE (1:2)

CISPLATIN

CITRUS RED NO.2

COAL-TAR PITCHES

CRESIDINE

CRESIDINE

TRESOTES

UPFERRON

CYCASIN

CYCLOPHOSPHAMIDE

DAUNOMYCIN

DDT

DECARBAZINE

DI(2-ETHYLHEXYL)PHTHLATE

DIACETYLBENZIDINE

2,4-DIAMINOANISOLE

2,4-DIAMINOANISOLE SULFATE

4,4'-DIAMINOOIPHENYL ETHER

2,4-DIAMINOTOLUENE

DIBENZ (A, H)ACRIDINE

DIBENZ (A, H)ANTHRACENE

DIBENZ (A, J)ACRIDINE

DIBENZO(A,E) PYRENE

DIBENZO(A,H) PYRENE

DIBENZO( $(A, d)$ PYRENE

DIBENZO $(A, L)$ PYRENE

1,2-DIBROMO-3-CHLOROPROPANE

1,2-DIBROMOETHANE

3,3'-DICHLORO-4,4'-DIAMINO-DIPHENYL ETHER

1,4-DICHLOROBENZENE

DICHLOROBENZENE

3,3'-DICHLOROBENZIDINE

3'-DICHLOROBENZIDINE DIHYOROCHLORIDE
PARA-CHORO-ORTHO-TOLUIDINE

METHYL-CCNU

CCNU

TRICHLOROMETHANE

METHYL CHLOROMETHYL ETHER; CHLOROOIMETHYL ETHER
$563-47-3$

$95-83-0$

$95-69-2$

13909-09-6

$13010-47-4$

$67-66-3$

$107-30-2$

$11103-86-9$

$13765-19-0$

$7789-00-6$

$7775-11-3$

$7758-97-6$

$7789-06-2$

$15430-94-6$

$13530-65-9$

$14986-48-2$

$14977-61-8$

1333-8-20

15663-27-1

6358-53-8

PARA-CRESIDINE

PARA-CRESIDINE

65996-93-6

120-71-8

120-71-8

8001-58-9

135-20-6

14901-08-7

50-18-0

20830-81-3

50-29-3

4342-03-4

$117-81-7$

$613-35-4$

615-05-4

$39156-41-7$

101-80-4

95-80-7

226-36-8

53-70-3

$224-42-0$

189-64-0

192-65-4

189-55-9

191-30-0

96-12-8

106-93-4

$28434-86-8$

106-46-7

106-46-7

$91-94-1$

$612-83-9$ 


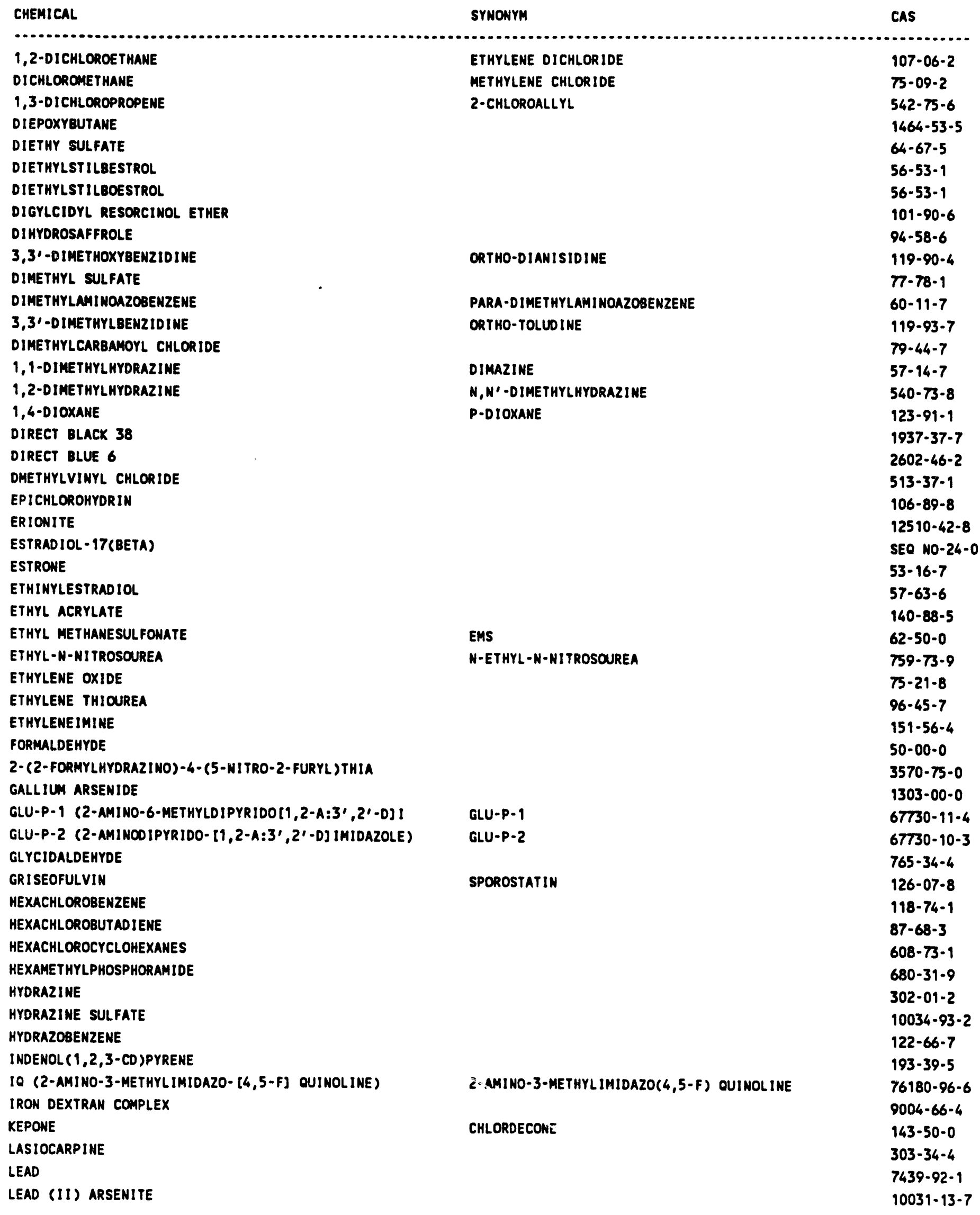


CHEMICAL

SYNONYM

CAS

LEAD (II) PHOSPHATE

LEAD ACETATE (11), TRIHYDRATE

LEAD CHROMATE (VI) OXIDE

LINDAME

MEA-ALPHA-C (2-AMINO-3-METHYL-9H-PYRIDOL2,3-B] INDO

MEDROXYPROGESTERONE ACETATE

MELPHALAN

2-AMINO-3-METHYL-A-CARBOLINE

PERLUTEX

$7446-27-7$

$6080-56-4$

$18454-12-1$

$58-89-9$

$68006-83-7$

$71-58-9$

MERCURY (11) 0-ARSEMATE

$148-82-3$

MESTRANOL

8-METHOXYPSORALEN

8-METHOXYPSORALEN PLUS ULTRAVIOLET RADIATION

METHYL CHLOROMETHYL ETHER

$7784-37-4$

$72-33-3$

8-METHOXYSALEN, METHOXSALEN

298-81-7

METHYL METHANESULPHONATE

METHOXSALEN PLUS ULTRAVIOLET RADIATION;PUVA

2-METHYL-1-NITROANTHRAOUINONE

METHYL - N' - NITRO-N-NITROSO-GUANIDINE

METHYL-N-NITROSOUREA

METHYL-N-NITROSOURETHAME

2-METHYLAZIRIDINE

METHYLAZOXYMETHANOL

METHYLAZOXYMETHANOL ACETATE

5-METHYLCHRYSENE

4,4'-METHYLENE BIS(2-CHLOROANILINE)

4,4'-METHYLENE BIS(2-METHYLANILINE)

4,40 -METHYLENEBIS (N.N-DIMETHYL ) BENZENEMAMINE

4,4'-METHYLENEDIANILINE

4,4'-METHYLENEDIANILINE DIHYDROCHLORIDE

AETHYLTHIOURACIL

METRONIDAZOLE

MICHLER'S KETONE

MIREX

MITOMYCIN C

MONOCRORTALINE

MUSTARD GAS

NAFENOPIN

1- MAPHTHALENE

2- MAPHTHYLAMINE

NICKEL

NICKEL (II) OXIDE (1:1)

MNNG

$107-30-2$

$66-27-3$

129-15-7

$70-25-7$

N-METHYL - N-NITROSOUREA

$684-93-5$

N-METHYL-N-NITROSOURETHANE

615-53-2

PROPYLENEIMINE

1-HYDROXYMETHYL-2-METHYLDITHIMIDE-2-OXIDE

$75-55-8$

590-96-5

$592-62-1$

$3697-24-3$

MOCA;MBOCA

$101-44-4$

838-88-0

$101-69-1$

$101-77-9$

$13552-44-8$

56-04-2

$443-48-1$

TETRAMETHYLOIAMINOBENZOPHENONE

$990-94-8$

FERRIAMICIDE; DECHLORANE 4070

$2385-85-5$

$50-07 \cdot 7$

$315-22-0$

SULFUR MUSTARD

505-60-2

ALPHA-NAPHTHYLAMINE

$3779-19-5$

$134-33-7$

BETA-NAPHTHYLAMINE

$91-59-8$

$7440-02-0$

$1313-99-1$

$12125-56-3$

$12255-80-0$

NICKEL ARSENIDE

NICKEL ARSENIDE

NICKEL ARSENIOE SULFIDE

NICKEL CARBONYL

NICKEL REFINERY DUST

NICKEL SULFIDE

NICKEL (A) ACETATE (1:2)

12256-33-6

$12255-10-8$

$13443-30-3$

NICKEL(II) CARBOWATE (1:1)

$12035-72-2$

$373-02-4$

NICKEL(II) HYDROXIDE

NIRIDAZOLE

NITRILOTRIACETIC ACID

[4(5, - NITRO-2-FURYL)-2-THIAZOLYL] ACETAMIDE

-NITRO-O-ANISIDINE

NIFURADENE

$3333-67-3$

$12064 \cdot 45-7$

$61-57-4$

$139-13-9$

$42011-48-3$

$99-59-2$ 
NITROGEN MUSTARD HYOROCHLORIDE

$51-75-2$

$55-86-7$

NITROGEN MUSTARD N-OXIDE

2-NITROPROPAME

MITROSO-N-ETHYLUREA

NITROSO-N-METHYLUREA

NITROSCOI-N-BUTYLAMIME

NITROSCOI-N-PROPYLAMIME

MITROSCOIETHANOLAMIME

MITROSODIETHYLAMINE

NITROSCO IMETHYLAMINE

NITROSCOIPHENYLAMINE

3-N-NITROSOMETHYLAMINO) PROPIONITRILE

4-N-NITROSOMETHYLAMINO)-1-(3-PYRIOYL)-1-BUTANONE

NITROSOMETHYLETHYLAMINE

NITROSOMORPHOL IME

NITROSONORNICOTINE

NITROSOPIPERIDINE

NITROSOPYRROL IDINE

NITROSOSARCOSINE

MORETHISTERONE

OIL ORANGE SS

4,4 '-OXYDIANILINE

OXYMETHOLONE

PANFURAN S (CONTAINING DIHYDROXYMETHYLFURATRIZINE)

PHENACETIN

PHENAZOPYRIDINE HYDROCHLORIDE

PHENOBARBITAL

PHENOXYBENZAMINE HYDROCHLORIDE

PHENYTOIN

POLYBROMINATED BIPHENYL

POLYCHLORIMATED BIPHENYL

POLYCHLORINATED BIPHENYL

PONCEAU 3R

PONCEAU MX

POTASSIUN BROMATE

PROCARBAZINE HYDROCHLORIDE

PROGESTERONE

$N$-NITROSO-N-ETHYLUREA

$126-85-2$

$79-46-9$

$759-73-9$

$N$-MITROSO-N-METHYLUREA

$684-93-5$

$N$-NITROSCOI-N-BUTYLAMINE

N-NITROSO-N-PROPYLAMIME

N-MITROSOOIETHANOLAMINE

N-NITROSODIETHYLAMINE

N-NITROSOD IMETHYLAMINE

PARA-NITROSOOIPHENYLAMINE

NAK

N-NITROSOMETHYLETHYLAMINE

N-WITROSOMORPHOLINE

N-NITROSONORNICOTINE

N-NITROSOPIPERIDINE

N-NITROSOPYRROLIDINE

W-NI TROSOSARCOSINE

1-(0-TOLYLAZO)-2-NAPHTHOL

$4,4 \cdot$-OXYDI-ANILINE

FIREMASTER FF

PCB; AROCLOR 1260

PCB; AROCLOR 1254

1,3-PROP IOLACTONE
$924-16-3$

$621-64-7$

$1116-54-7$

$55-18-5$

$62-75-9$

156-10-5

$60153-49-3$

$64091-91-4$

10595-95-6

59-89-2

16543-55-8

$100-75-4$

930-55-2

13256-22-9

68-22-4

$2646-17-5$

$101-80-4$

434-07-1

$794-93-4$

$62-44-2$

$136-40-3$

50-06-6

$63-92-3$

$57-41-0$

$67774-32-7$

$11097-69-1$

11097-69-1

3564-09-8

$3769-53-3$

7758-01-2

$366-70 \cdot 1$

57-83-0

$1920-71-4$

$57-57-8$

$75-56-9$

51-52-5

50-55-5

$81.07-2$

$94-59-7$

$7446-34-6$

SELENIUN SULFIDE

SHALE-OILS

SILICA, CRYSTALLINE 
CHEMICAL

SYNOWYM

CAS

SOOIUN ORTHO-PHEHYLPHENATE

2-BIPHENYLOL, SOOIUM SALT

$132-27-4$

STEPTOZOTOCIN

STERICOMTOCYSTIN

$18883-66-4$

STYRENE

STYRENE OXIDE

$10048-13-2$

SULFALLATE

2,3,7,8-TETRACHLOROOIBENZO-P-DIOXIN

TCCO

TETRACHLOROETHYLENE

THIOACETAMIOE

PERCHLOROETHYLENE

$100-42 \cdot 5$

$96-09-3$

$95-06-7$

$1746-01-6$

$127-18-4$

$4,41-$ THICOIANILINE

$62 \cdot 55 \cdot 5$

THIOUREA

THORIUN DIOXIDE

TOLUEME DIISOCYAMATE

TOLUIDINE

TOLUIDINE HYOROCHLORIDE

TOXAPHENE

ORTHO-TOLUIDINE

ORTHO-TOLUIDINE HYOROCHLORIDE

139-65-1

$62-56-6$

$1314-20-1$

$548-84-9$

$95-53-4$

POLYCHLORINATED CAMPHENES

$636-21-5$

$1,3,4$-OXADIAZOLE

$8001-35-2$

TRANS-2 [CDIMETHYLAMINO)METHYL - IMINO] - 5 [2(5-NITRO-2

TREOSULPHAN

2,4,6-TRICHLOROPHENOL

TRICHLOROTOLUENE

TRIS(1-AZIRIDINYL)PHOSPHINE SULFIDE

TRIS(2,3-DIBROMOPROPYL) PHOSPHATE

THIOTEPA

55738-54-0

299-75-2

88-06-2

98-07-7

$52-24-4$

$126-72-7$

TRP-P-1 (3-AMINO-1, 4-DIMETHYL-5H-PYRIDO[4,3-B] INDO

TRIS

$62450-06-0$

TRP-P-2 (3-ANINO-1-METHYL-5H-PYRIDO [4, 3-B] INDOLE

TRYPAN BLUE

DIRECT BLUE 14

$62450-07-1$

$72 \cdot 57-1$

$66-75-1$

$59 \cdot 79-6$

593-60-2

$75-01-4$

$106-87-6$

$2,4-X Y L I D E N E$

$95-68-1$ 


\section{Appendix 12}

Carcinogen Exposure Assessment Survey Form \&

Industrial Hygiene Hazard Evaluation Form 


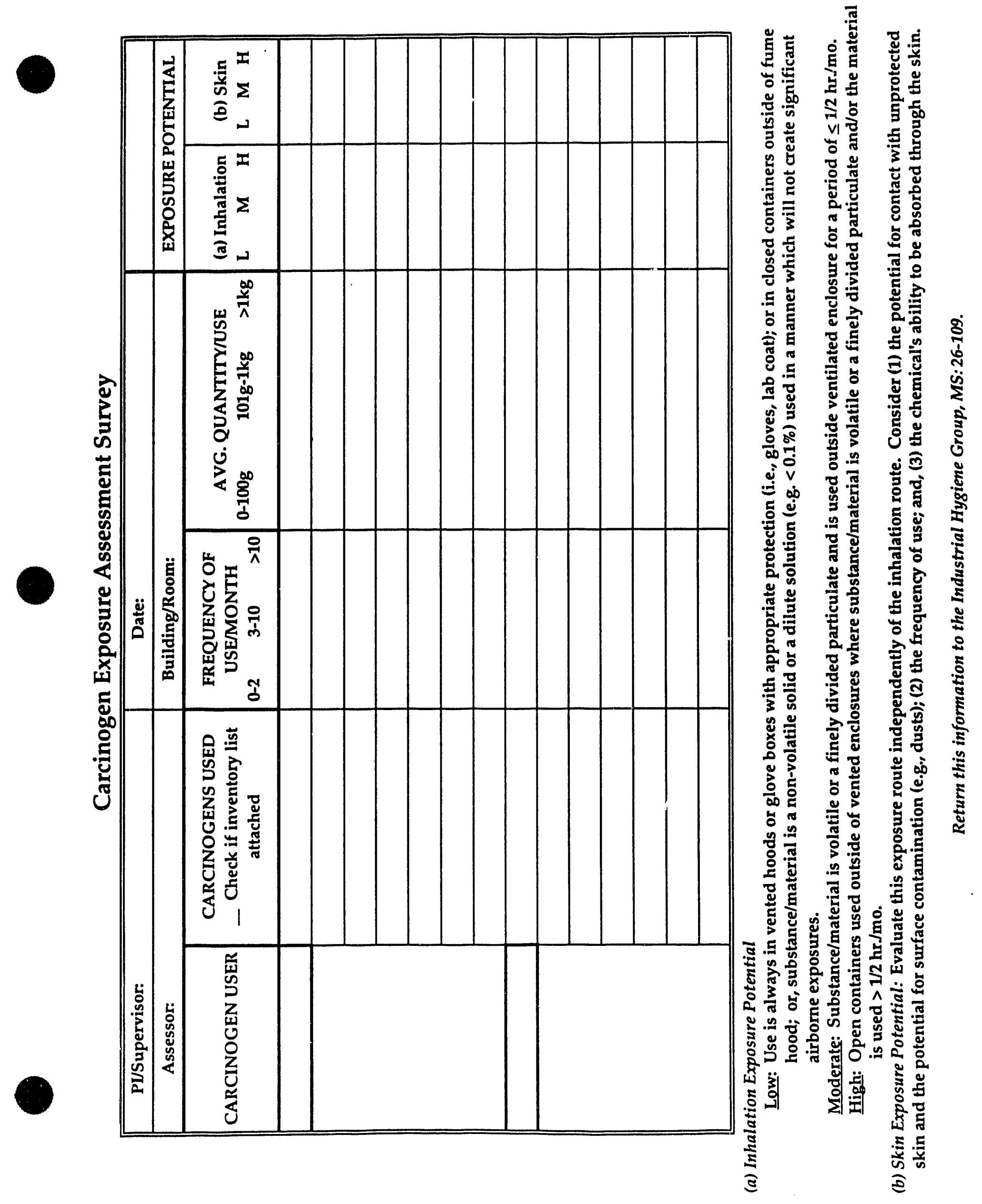




\section{LBL INDUSTRIAL HYGIENE GROUP}

HAZARD EVALUATION FORM

Name:

Emp. No:

Date:

Group:

Bldg. No:

$\mathrm{R} \mathrm{m}:$

Ext:

Hazardous Materials / Agents:

Issue / Operations / Condition:

Actions Needed / Taken:

By: 
Appendix 13

Reproductive Toxins 
Appendix 13

\section{Reproductive Toxins*}

\section{Developmental toxins:}

\section{Chemical}

acetohydroxamic acid

CAS Number

all-trans retinoic acid.

546883

alprazolam

302794

amikacin sulfate

28981977

aminoglutethimide

39831555

aminopterin

125848

.54626

benomyl

17804352

benzphetamine hydrocholoride

5411223

bischloloroethyl nitrosourea (BNCU) (Carmustine)

154938

bromoxynil

1689845

1,4-butanediol dimethylsulfonate (Busulfan)

55981

carbon disulfide. 75150

carboplatin 41575944

chenodiol

474259

chlorcyclizine hydrochloride.

1602219

chlorambucil

305033

chlordecone (Kepone)

143500

1-(2-chloroethyl)-3-cyclohexyl-1-nitrosourea (CCNU).....13010474

clomiphene citrate.

50419

conjugated estrogens

cyanazine

21725462

cycloheximine.

.66819

cyclophosphamide (anhydrous)

50180

cyclophosphamide (hydrated).

6055192

cyhexatin

13121705

cytarabine

147944

danazol

17230885

daunorubiciin hydrocholoride

23541506

diethylstilbestrol (DES)

56531

dinocap

39300453

dinoseb

88857

diphenylhydantoin (phenytoin)

54410

doxycycline 
Chemical

CAS Number

ergotamine tartrate

379793

ethylene glycol monoethyl ether

ethylene glycol monomethyl ether

etoposide

etretinate

54350480

fluorouracil

fluoxymesterone

flutamide

halazepam 23092173

hexachlorobenzene.

ifosfamide .3778732 iodine-131

lead

lithium carbonate

lithium citrate

medroxyprogesterone acetate

71589

megestrol acetate 595335

melphalan 148823

menotropins 9002680

mercaptopurine .6112761

mercury and mercury compounds

methacycline hydrochloride.

3963959

methimazole 60560

methotrexate 59052

methotrexate sodium

methyl mercury

midazolam hydrochloride

misoprostol 62015398

mitroxantrone hydrochloride 70476823

nafarelin acetate 86220420

netilmicin sulfate 56391572

nitrogen mustard (mercloroethamine) 51752 
Chemical CAS Number

nitrogen mustard hydrochloride

(mechlorethamine hydrochloride) ..................................55867

norethisterone (norethindrone).............................................68224

norethisterone (norethindrone)/ethinyl estradiol ....68224/57636

norethisterone (norethindrone)/mestranol.................68224/72333

norgestrel

6533002

oxytetracycline

.79572

paramethadione......................................................................115673

penicillamine..................................................................................52675

pentobarbital sodium .....................................................................57330

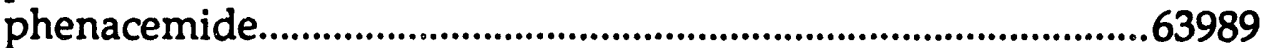

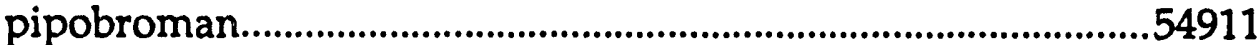

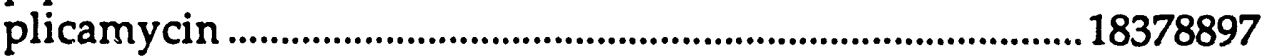

polychlorinated biphenyls

procarbazine hydrocholride..........................................................366701

propylthiouracil ..................................................................................51525

streptomycin sulfate.......................................................................3810740

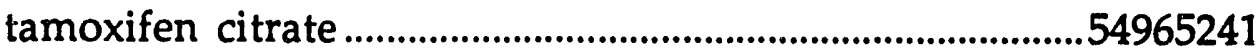

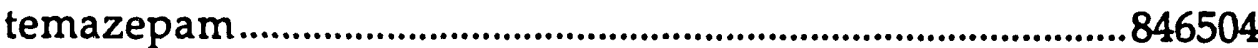

testosterone enanthate.............................................................515377

2,3,7,8-tetrachlorodibenzo-para-dioxin (TCDD)....................1746016

tetracycline hydrochloride .....................................................64755

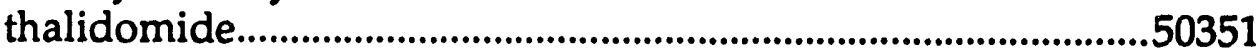

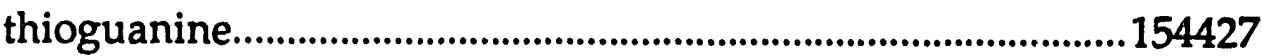

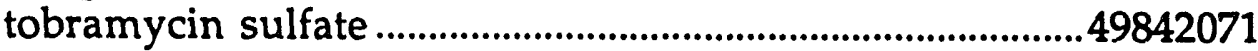

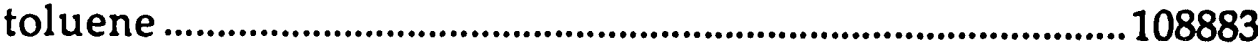

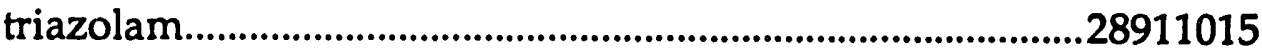

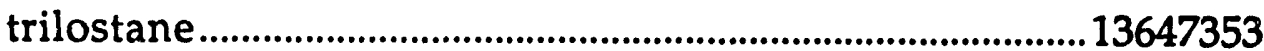

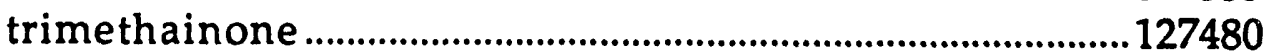

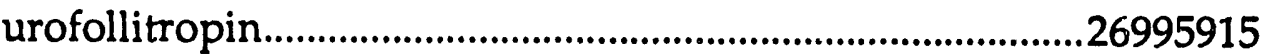

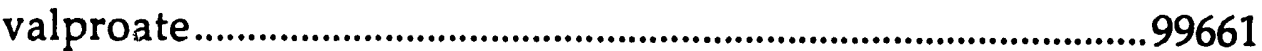

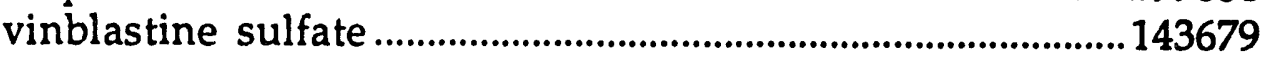

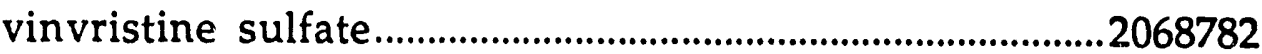

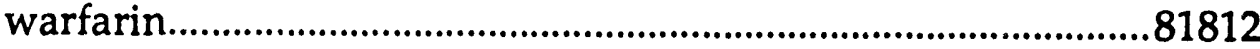

App. 13 - 3 


\section{Chemical}

\section{CAS Number}

\section{Female reproductive toxins:}

aminopterin 54626 anabolic steroids

carbon disulfide 75150

cyclophosphamide (anhydrous)

cyclophosphamide (hydrated) 6055192

ethylene oxide .75218

lead

3. Male reproductive toxins:

anabolic steroids

benomyl 178043252

carbon disulfide 75150 cyclophosphamide (anhydrous) 50180 cyclophosphamide (hydrated) 6055192

1,2-dibromo-3-chloropropane 96128 $\mathrm{m}$-dinitrobenzene .99650 o-dinitrobenzene 528290

p-dinitrobenzene 100254 dinoseb 88857

ethylene glycol monoethyl ether. 110805 ethylene glycol monomethyl ether. 109864

lead

"Extracted from the State of California Safe Drinking Water and Toxic Enforcement Act (Proposition 65) list of chemicals known to the State to cause reproductive toxicity.

Note: Many of the listed chemicals are medicines and other chemotherapeutic agents that cause adverse reproductive effects when consumed orally or when injected into the body. The potential for reproductive toxicity resulting from other routes of exposure common to the laboratory use of chemicals (i.e., inhalation and skin absorption) may be negligible. On the other hand, laboratories and shops 
should consider, at a minimum, the following chemicals known to cause reproductive effects when inhaled:

carbon disulfide

lead and lead compounds

dinitrobenzene

ethylene glycol monoethyl ether mercury and mercury compounds ethylene glycol monomethyl ether toluene ethylene oxide 
Appendix 14

Safety Training Check List 


\section{Appendix 14 \\ Safety Training Check List}

\section{Introduction}

It is the responsibility of Division management and line supervisors to train employees in the safety rules of the laboratory and to foster in them an attitude that day-to-day safety is an integral part of all experimental work. The purpose of this checklist is to provide guidance to supervisors for on-the-job safety orientation and training for new and current employees and participating guests. Employee and supervisor signatures at the end provide a record of hazard review.

\section{It is important to:}

- Review general laboratory safety rules and specific hazards with the new employee using the attached summary.

- Make arrangements for new employees to attend necessary training, listed in the Course Information bulletin. Call x5514 or x5251 for information.

- Make sure that the employee knows about the Material Safety Data Sheets and other safety references, their purpose and their locations.

- Make sure the employee knows that in the event of an emergency, help should be sought at LBL by dialing $x 7911$.

\section{Instructions:}

All new, or transferred employees are to review this checklist with their immediate supervisor in the first week of work. The employee and the supervisor will sign the checklist and a copy shall be retained in the employee's file for as long as he/she works in that lab. Upon termination or transfer, a copy shall be sent to personnel for permanent retention in the employee's file.

\section{Table of Contents:}
A. General Safety Information
B. Hazard Training
C. Work Practices and Procedures
D. Equipment/Machinery
E. Electrical Safety
F. Housekeeping 


\section{A. General Safety Information}

Yes No

I understand safety responsibilities and authorities at LBL:

- personal

- my supervisor

- EH\&S

I know what PUB-3000 is.

I know where PUB-3000 is.

I know what the Chemical Hygiene \& Safety Plan (CHSP) is.

I have been designated as a Radiation Worker.

I participate in the following radiation exposure monitoring programs:

- Radiation Dosimeter:

- - $\quad$ gamma only

- - Bioassay

- _ I know that there is no eating and drinking in labs/shops.

- I know that no smoking is allowed in labs.

— _ I know my responsibilities regarding Hazards Communication.

- _ I am responsible for safety training for others.

_ _ I know where to find MSDS for chemicals/materials.

- - I know what an Operational Safety Procedure (OSP) is, what a Specific Safety Procedure (SSP) is, and when each is required.

_ _ I know who the Division Safety Coordinator is.

- _ I know my assembly area in an emergency.

- _ I know where to find the Laboratory Emergency Plan.

— _ I know about the LBL Bioassay Program. 
Yes No

I know where emergency numbers are posted.

I know where hazard signs are posted - proper label used.

I know Workers' Compensation Procedures, and will report any illness or injury to my supervisor and Health Services.

\section{B. Hazard Training}

I have been instructed in the handling/use of the following hazardous materials and physical hazards.

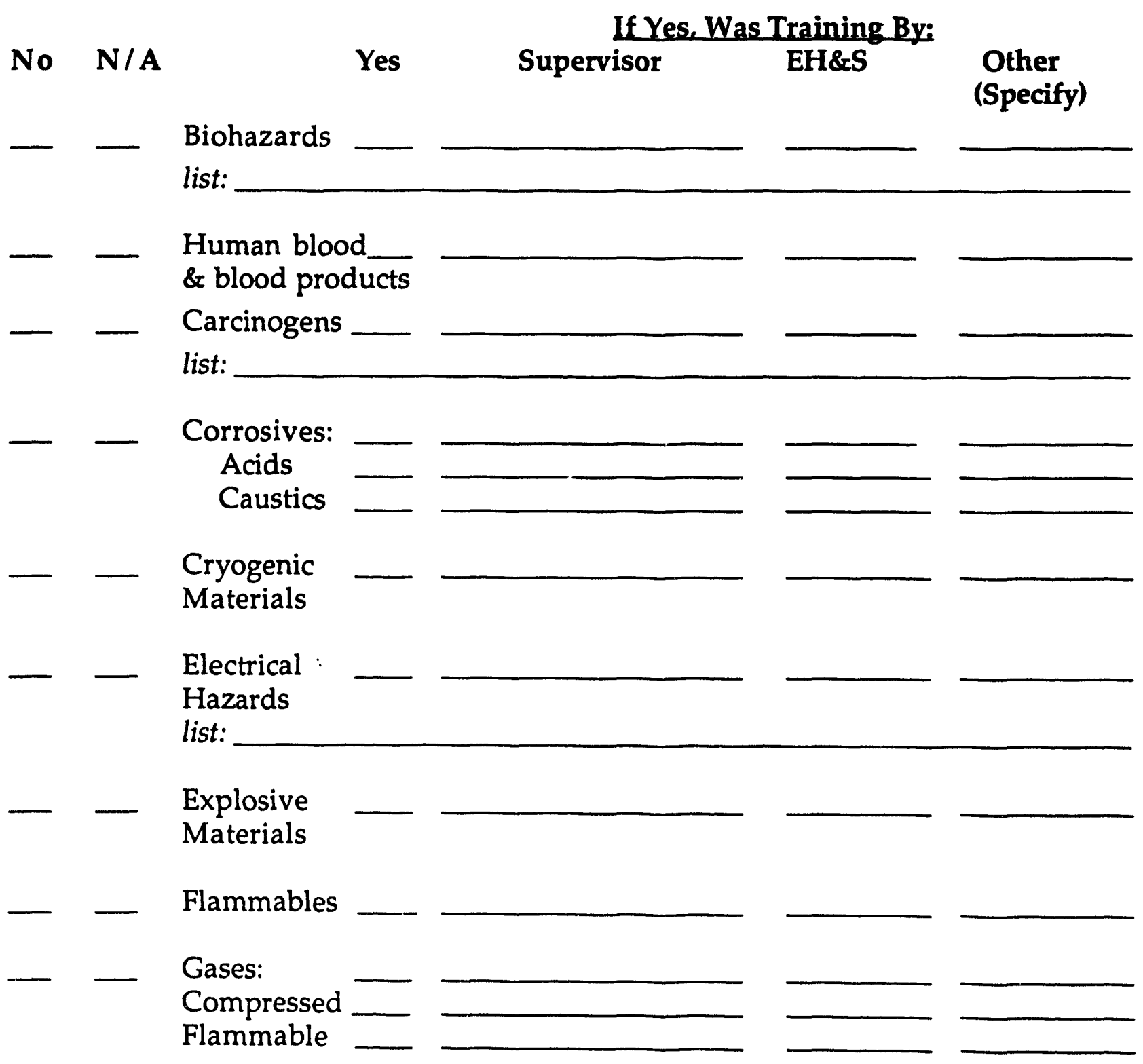

App. 14 - 3 


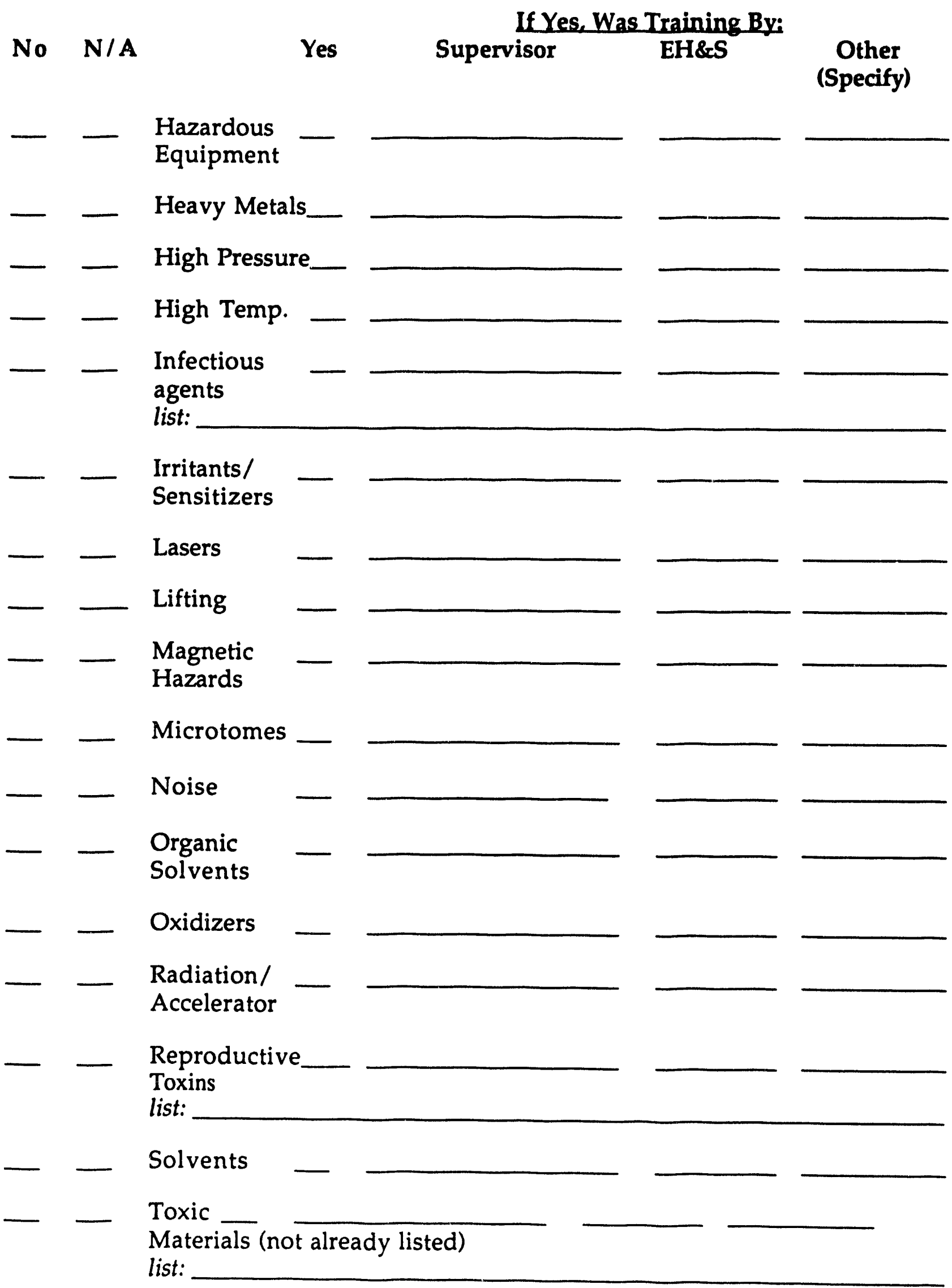


No N/A

Yes

If Yes, Was Training By: Supervisor EH\&S

Other

(Specify)

WASTE:

Medical/

Biohazardous

- - $\begin{aligned} & \text { Chemical } \\ & \text { Radioactive - } \\ & \text { Sharps }\end{aligned}$

Others:

\section{Work Practices and Procedures}

I have been instructed in the following work practices and procedures.

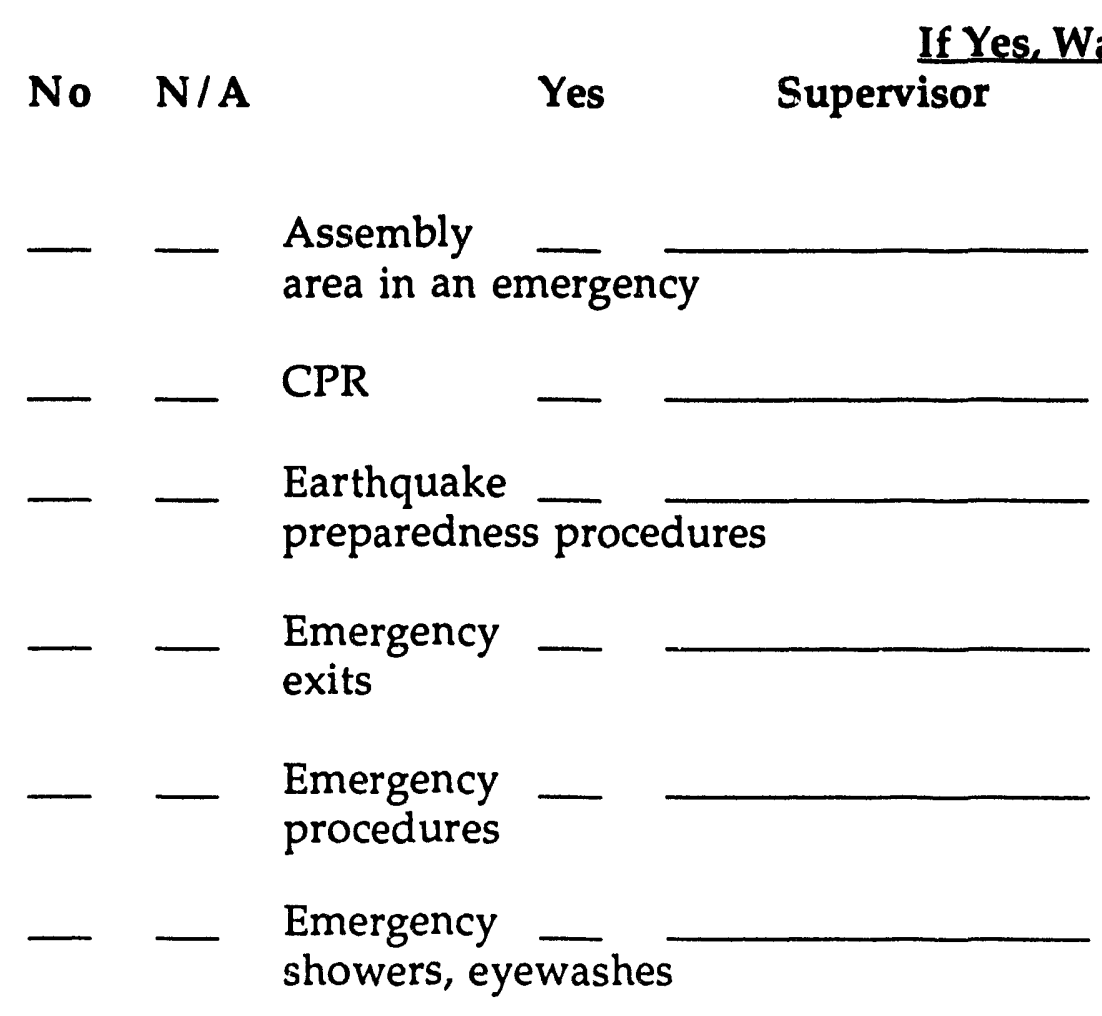

App. 14 - 5 
No N/A

Yes

If Yes, Was Training By:

Supervisor

EH\&S

Other

(Specify)

Fire

extinguishers (location and use)

Laboratory

Emergency Plan

Lockout-Tagout

Spill kits -

location and use

Standard

First Aid

Ignition

sources around flammable materials

$-\quad-\quad \begin{aligned} & \text { Appropriate } \\ & \text { chemical storage }\end{aligned}$

Transporting

chemicals safely

Confined

Space Entry

Not working

alone (Always let someone know where you are and how $\overline{\text { long you'll }}$ be working)

Fume hoods

- correct use and performance checks

Labelling of

chemical containers

Pipetting -

mechanical (never by mouth)

Waste

Minimization

App. 14 - 6 


\section{Equipment/Machinery}

I have been trained in the use of the following equipment.

No N/A

Yes

If Yes, Was Training By:

Supervisor

EH\&S

Dther

(Specify)

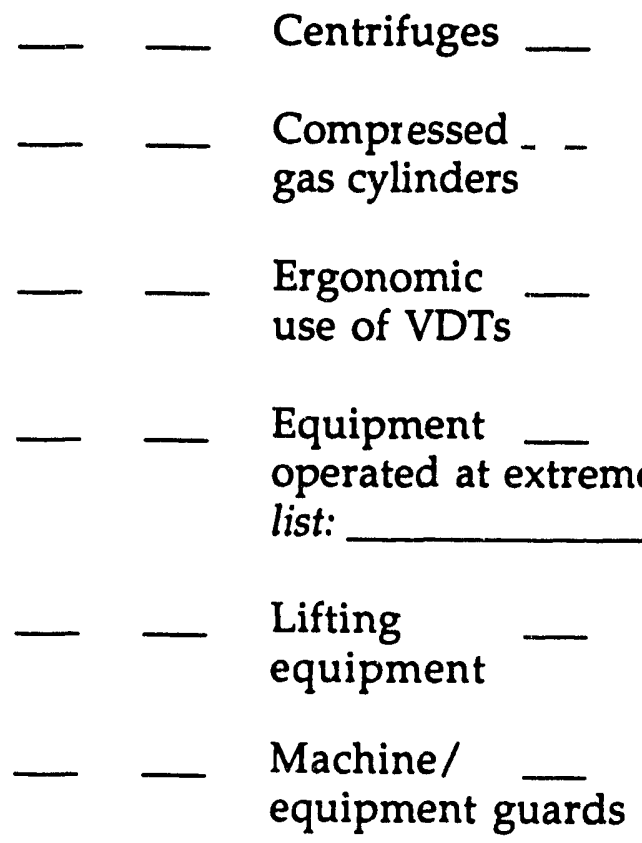

- - Sterilization

- $\quad$ Radiation monitoring equipment

UV lights

Vacuum systems

$\mathrm{X}$-ray sources

Machine

Tools

Soldering, welding, cutting

Syringes \& needles

App. 14 - 7 
No N/A

Yes

If Yes, Was Training By:

Supervisor

EH\&S

Other

(Specify)

Use of power

tools and/or shop/industrial machinery

list:

Shop/

industrial machinery (other, not already listed)

list:

Others:

\section{E. Electrical Safety}

I am aware of the following regulations:

Yes No N/A

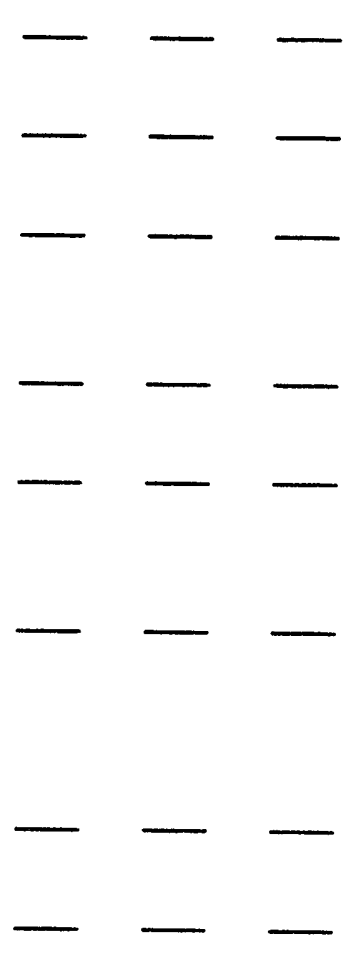

All boxes, raceways, fittings, etc., must have protective covers.

Cable trays are to be properly grounded; not overfilled.

Disconnect devices (switches, breaks, etc.) are to be identified as to use.

Electrical cords/wires are to be protected from damage.

Electrical equipment is to be grounded or provided with barriers/shields.

Electrical equipment that is provided should be suitable for workplace environment (i.e., proper electrical classification for wet or explosive areas, etc.).

Exposed terminals are to be guarded adequately.

Ground fault interrupters (GFI) are installed in wet areas and in receptacles within 6 feet of sinks, including fume hood sinks. 
Yes No N/A

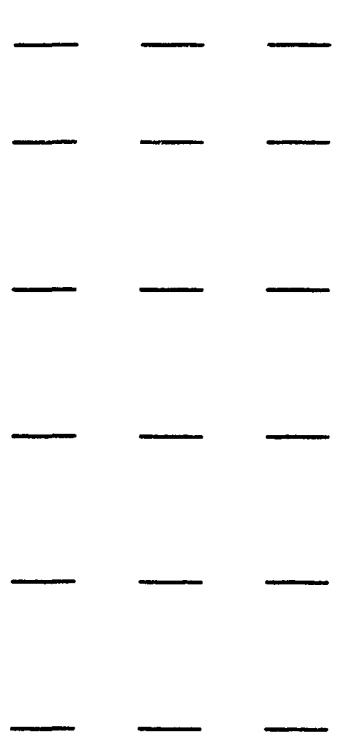

Grounding points are to be clearly identified inside enclosures.

Hand-held power tools are to be provided with double insulation or electrical grounds.

High voltage control panels and doors are to be closed and interlocked. Power cabinets are to be properly labeled.

High voltage equipment grounding hooks are available and fully visible.

High voltage warning signs are to be posted at enclosure doors and removable panels as required (voltage greater that 600 volts).

Lock out tags should be on circuit breaks, unused circuit breakers should be locked out and tagged.

No broken or removed grounding pins are to be present on 3wire cord plugs.

No metal ladders are to be near electrical equipment, or to be used for electrical work.

Office wiring should be sufficient to meet electrical needs.

Oil-filled transformers are to be provided with a secondary containment.

Permanently located machinery is to be hard wired.

Plugs, cords (no frayed cords) and receptacles are to be properly installed and in good condition. They must have cover plates on receptacles.

There is unobstructed access to control switches, circuit breakers, electrical panels (3 foot clear zone).

Proper use of power extension cords:

1. Not using them in place of permanent wiring

2. Not running through walls, ceilings, doors, etc.

3. Use of proper cord plugs

4. Use of three conductor cables

5. No use of damaged or taped cords

6. Not daisy-chaining them together 
F. Housekeeping

I am aware of the following general needs/requirements.

Yes No N/A

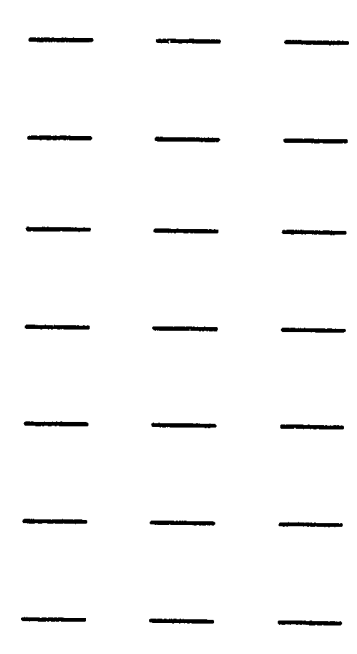

Aisles, passageways have $3 \mathrm{ft}$. of clearance.

Floors are clean and dry.

Furniture is in good repair.

Storage areas are orderly.

Storage shelves have lips to prevent objects from falling.

Houseplants are properly secured.

Locker, file cabinets, furniture, and equipment over 5 feet high are secured against tipping and falling during an earthquake. Seismic tiedown has been completed.

No heavy objects are stored above 5 feet unless secured.

No cardboard boxes, piles of papers, or computer output are stored under tables.

No food or beverages are in chemical use/storage areas.

No sharp, protruding objects are left unprotected.

No trip hazards exist.

There is an orderly and clean appearance to the work area.

Raised areas (platforms) which are used for storage are identified as to load limit. Loads are within limits.

Refrigerators are properly labeled when used for food or beverage storage.

Separate storage and disposal containers are available for rags, glass, paper, and hazardous materials.

There is sufficient illumination of work areas. 
Yes No N/A

_ _ _ Unused equipment is maintained in a safe, orderly manner or is sent to storage/salvage.

When materials are stored, they are securely stacked to prevent tipping.

I understand that my supervisor will keep me trained as new hazards are introduced into the workplace, and that I can ask my supervisor or EH\&S for further health and safety information as the need arises.

Any comments:

Employee's signature:

Date:

Supervisor's signature:

Date: 


\section{Appendix 15}

\section{Chemical Information}

(MSDSs, Ethernet, and TOMES) 
Appendix 15

Using the Material Safety Data Sheet (MSDS)

\section{INTRODUCTION}

The purpose of the MSDS is to provide vital information on health and physical hazards. This part of the program will illustrate and describe each section of the MSDS to help you understand the data. You can use this information to plan training programs and to explain the MSDS to your employees. Some of the terms on the MSDS are quite technical; refer to the Glossary of Terms (Appendix Thirteen) for help.

The MSDS must include, at a minimum, all eight of the described sections. The style and layout may vary. However, every section must be filled in, even if the item is not applicable (indicated by N/A). There should be no blank spaces! Note that some of the information, such as the chemical family, may be included, but is not required. Remember that the information on the MSDS is prepared by the manufacturer of the product. Therefore, some data sheets contain excellent information, some are adequate, and others are poor. Other sources of data on toxic and health effects should be consulted for more complete information.

\section{PRODUCT IDENTITIY}

Identity: The name of the product as it appears on the label. A product may be a mixture of two or more chemicals.

Manufacturer's Name, Address, and Phone Number: Self explanatory. If the data comes from a source other than the manufacturer, the actual source must be indicated. The date of preparation or revision must be indicated.

Emergency Telephone Number(s): 24-hour number(s) that the manufacturer provides, so that emergency information can be obtained (e.g., medical emergencies).

Chemical Family: The general class of compounds to which the hazardous substance or mixture belongs (e.g., ethers, acids, ketones, solvents). This term does not give you the exact content of the product.

Formula: The chemical formula may be given for single elements and compounds [e.g., sulfur dioxide (SO2), formaldehyde (HCHO)]. This is not the formulation for mixtures. 


\section{Using the Material Safety Data Sheet (MSDS), cont.}

\section{HAZARDOUS INGREDIENTS}

If the product is a mixture, all hazardous ingredients must be listed. However, ingredients that are not hazardous, or make up less than $1 \%$ of the product (less than $0.1 \%$ for carcinogens), do not have to be reported.

Exposure standards, i.e., Threshold Limit Value (TLV), and Permissible Exposure Limit (PEL) are included in this section, or under Health Hazards. Note the higher the number for a TLV or PEL, the less hazardous the substance. See the Glossary for definitions of TLV and PEL.

The \% column is intended to show the approximate percentage by weight or volume of each hazardous ingredient compared to the total weight or volume of the product. Normally, percentages will be listed to the nearest $5 \%$. When the substance constitutes less than $5 \%$ of the product, this is indicated.

CAS Number: Chemical Abstract Service registry number identifies specific chemicals only, not mixtures; it is optional.

\section{PHYSICAL DATA}

This section contains very important data to help predict the behavior of the material in experimental situations. The information provided is for the material as a whole, rather than for each hazardous ingredient. Vapor pressure, vapor density, $\%$ volatiles, and evaporation rate all basically tell you the same thing: whether breathing the vapors will be a problem, thus indicating the need for proper ventilation.

Boiling Point: The temperature at which a liquid changes to a vapor at a given pressure; usually in degrees Fahrenheit $\left({ }^{\circ} \mathrm{F}\right)$ at the sea-level pressure of $\mathbf{7 6 0}$ millimeters of mercury ( $\mathrm{mm}$ of $\mathrm{Hg}$ ). For mixtures, the initial boiling point or the boiling range may be given. A low boiling point may be a special fire hazard.

Vapor Pressure: Refers to the pressure exerted by a saturated vapor above its own liquid, usually stated in $\mathrm{mm}$ of $\mathrm{Hg}$ at $250 \mathrm{C}(77 \mathrm{oF})$. The lower the boiling point, the higher the vapor pressure. A high vapor pressure indicates easy evaporation.

Vapor Density: Tells whether the material is heavier or lighter than air. This is useful information to indicate a confined-space hazard. If heavier than air, the material will concentrate in low places, such as floors, elevator shafts, sewers, or the bottom of tanks. 


\section{Using the Material Safety Data Sheet (MSDS), cont.}

\% Volatiles by Volume: How much of the material evaporates at room temperature. A substance that is $100 \%$ volatile, will evaporate completely, leaving no residues.

Evaporation Rate: The rate at which the material will evaporate when compared to the rate of evaporation of a known material, usually butyl acetate. If another material is used for comparison, it should be indicated. If the number is greater than 1 , the product evaporates more easily than the comparison material.

Solubility in Water: The percentage of a material (by weight) that will dissolve in distilled water, at room temperature.

Specific Gravity: The ratio of the weight of a volume of material to the weight of an equal volume of water. For insoluble materials, a specific gravity of less than one means the material is lighter than water and will float. Greater than one means it sinks in water.

Melting Point: The temperature at which a solid becomes a liquid under normal room conditions.

Appearance and Odor: A brief description of the material at normal room temperature and atmospheric conditions. Do not rely on odor to alert you to a dangerous exposure. Some substances can reach hazardous levels and have no noticeable odor.

\section{FIRE \& EXPLOSION HAZARD DATA}

This section should clearly indicate whether the material is flammable. If it is flammable, make sure there are no ignition source nearby and that you have the correct fire extinguisher on hand. If you work with solvents, peroxides, explosives, metal dusts, or other unstable substances, this section is very important.

Flash Point: The lowest temperature at which the material gives off enough vapor to ignite; this will help determine storage and handling procedures. The method used to obtain this information should be stated (e.g., closed cup).

Flammable or Explosive Limits: The range over which a flammable vapor, when mixed with the proper proportions of air, will flash or explode if ignited. The range is designated by lower explosive limit (LEL) and upper explosive limit (UEL), and is expressed in \% of volume of vapor in the air.

Extinguishing Media: Indicates what type of fire extinguisher to use, such as water, fog, foam, alcohol foam, carbon dioxide, or dry chemical. 


\section{Using the Material Safety Data Sheet (MSDS), cont.}

Special Firefighting Procedures: Special handling procedures, personal protective equipment, and unsuitable firefighting substances should be listed. For example, water should not be used on fires involving reactive metals. General firefighting methods are not described.

Unusual Fire and Explosive Hazards: Hazards that might occur as a result of overheating or burning of the material, including any chemical reactions or change in chemical form or composition.

\section{REACTIVITY DATA}

This section indicates how unstable the substance is and lists conditions to avoid to prevent dangerous reactions. This information will help you handle and store the material properly.

Stability: The checked box will indicate whether the material is stable or unstable and under what conditions instability occurs.

Incompatibility: Lists materials and conditions to avoid. Such conditions may include extreme temperatures, jarring, or inappropriate storage. This is important to determining what other chemicals the material cab be stored/used with.

Hazardous Decomposition Products: A list of the hazardous materials that may be produced if the material is exposed to burning, oxidation, heating, or certain chemical reactions. The product shelf life should be included, when applicable.

Hazardous Polymerization: Polymerization is a chemical reaction in which two or more molecules of a substance combine to form repeating structural units of the original molecule. A hazardous polymerization causes an uncontrolled release of energy (heat). If this reaction can occur, it must be indicated.

\section{HEALTH HAZARD DATA}

This section lists routes of entry (inhalation, skin absorption, ingestion), and gives signs and symptoms of overexposure, such as skin rash, tremors or dizziness. Shortterm (acute) and long-term (chronic) health hazards, such as the ability to cause cancer (carcinogenicity), birth defects (teratogenicity), or "target organ" damage, should be listed. Some products cause both types of effects. Unfortunately, this important section often lacks adequate information, especially on the health effects of long-term exposure. 
Using the Material Safety Data Sheet (MSDS), cont.

Instructions for first aid and emergency procedures for victims of acute inhalation, ingestion, or skin or eye contact must be included. Medical conditions that can be aggravated by exposure must also be stated.

Information on exposure standards, such as TLV, PEL, or STEL, and toxicity data (indicated by an LD50 number), may be included here. Toxicity data is only an estimate of the degree of toxicity, based on experiments with test animals.

\section{PRECAUTIONS FOR HANDLING}

This information will help you prepare for emergencies by having the proper materials and equipment on hand. This section lists methods, special equipment, and precautions necessary to control and clean up spills, leaks, and other releases. For example, if respirators are required to clean up a spill, that fact should be shown.

Acceptable waste-disposal methods, as well as prohibited methods, are described. The user will also be alerted to any potential environmental danger to the general population, crops, water supplies, etc.

Instructions for safe handling and storage, such as the warning not to store acids and bases together, may be given. Any additional special precautions not addressed elsewhere in the MSDS should also be listed here. These may include instructions for storage life or transportation, such as special packaging or temperature control.

\section{CONTROL MEASURES}

This section is essential for protecting employees from overexposure. It lists personal protective equipment, such as proper gloves, safety glasses, or respirators, ventilation necessary to work safely with the material, and work/hygienic practices. Types and descriptions of necessary equipment should be specified (e.g., organic vapor cartridge, neoprene gloves). If the material has a low TLV, indicating a dangerous health hazard, local ventilation is recommended, not general or dilution ventilation. Remember, engineering controls, such as the right kind of ventilation, are always preferable to relying on respirators. 


\author{
Canadian Centre for \\ Occupational Health and Safety
}

Ground drums and bond transter containers.

Keep containers closed when not in use. Avoid roleasing mist or vapour.

CLEAN-UP AND DISPOSAL

Only trainod porsonnel should clean up. Ensure appropriate ventilation is provided. Use appropriate protective clothing and respirators.

Remove or extinguish all bontion sources. Follow manuizeturer's recommendations for cloan-up and docontamination. Comply with erivironmental regulations.

\section{FRST ND}

Inhalation:

Ensure own safoty first.

Use "buddy" system.

Comtrol source of butylamine or move victim to irosh air.

Trainod porson may administer oxygen II physician advises.

\section{Eyo Contact:}

Flush affected eye(s) with lukewarm, pently llowing water for 20 minutes. holding the eyolid(s) open.

Skln Contact:

Remove contaminated clothing under nunning water.

Immediately wash aftected area with Ukowarm, genily nuning water for at bast 20 minutes.

Ingestion:

Never give anything by mouth if victim is:

- bsing consciousness

- unconscious

- convulsing

Pinse mouth thoroughly whth water.

Have victim drink about $250 \mathrm{~mL}(8$ 02.) of water.

DO NOT INDUCE VOMITING.

Note: Obtain medical aflention

IMMEDLATELY for all serious exposures. Consult a physician or the nearest Poison Control Centre.

NEED MORE INFORMATION?

So日 CHEMINFO record no. 454E, available from CCOHS. 
•TOMES Plus'"

Toxicology, Occupational Medicine \& Environmental Series

MICROMEDEX, INC. DA 


\section{TOXICOLOGY, OCCUPATIONAL MEDICINE \& ENVIRONMENTAL SERIES}

The TOMES Plus ${ }^{\text {'m }}$ Information System is a user-friendly, industrial chemical database which is continually updated and republished every 90 days in its entirety. It provides rapid, easy access to medical and hazard information needed for safe management of chemicals in the workplace, for evaluating exposures, for right-to-know issues for SARA Title III regulatory compliance and for quick response to emergency situations and environmental incidents. On-going enhancements make the TOMES Plus Information System the ultimate tool for occupational and environmental toxicity information. The components of the TOMES Plus database are as follows:

\section{MEDITEXT ${ }^{\text {m }}$ - Medical Managements}

Information for health care professionals involved in the evaluation and treatment of individuals acutely exposed to industrial chemicals. It als contains information necessary for reporting potential adverse health effects and treatment of chemical release exposures as required under Title III (Emergency Planning and Community Right to Know) of the Superfund Amendment and Reauthorization Act (SARA). MEDITEXT includes:

Overview

Chemical synonyms and potential use data

Clinical effects of exposure (indexed by organ system)

Laboratory evaluation of exposed individuals

Treatment of exposed individuals (organized by route of exposure)

Range of toxicity

Available forms and sources

Kinetics

Toxicology mechanisms

Standards

Physicochemical parameters

References

\section{HAZARDTEXT ${ }^{\text {m }}$ - Hazard Managements}

Information needed for the initial response to incidents involving hazardous materials such as spills, leaks and fires. It may be useful for medical and Hazmat first responders. HAZARDTEXT includes:

Chemical names and synonyms

Clinical effects

Treatment

Range of toxicity/standards

Hazard data and management

Physicochemical parameters

References 


\section{1st Medical Response Protocols}

Information for developing training programs or establishing protocols for first aid or initial response to accidents or ilinesses occurring in the workplace. It may be useful for occupational medicine and si ety personnel. Examples are amputations, burns, heat illness and seizures. 1st Medical Response Protocols include:

Presentation

Stabilization

Base contact

Special concerns

References

\section{RTECS from NIOSH}

Registry of Toxic Effects of Chemical Substances Database from the National Institute for Occupational Safety and Health

An annual compendium of toxicity data extracted from the scientific literature. Over 95,000 documents containing data on mutagenicity, carcinogenicity, reproductive hazards and acute and chronic toxicity of hazardous substances. RTECS documents include:

Substance identification

Synonym/trade name

Health hazard data

Acute toxicity

Irritation

Reproductive effects

Genetic effects

Standards and regulations

Status in the United States

Reviews

\section{HSDB from NLM}

Hazardous Stbstances Data Bank from the National Library of Medicine

Extensive review's of the toxicity and hazards of over 4,000 chemicals. HSDB documents include:

Administrative information

Substance identification

Manufacture/use information

Chemical \& physical properties

Safet; \& handling

Toxicity/biomedical effects

Pharmacology

Environmental fate/exposure potential

Expcsure standards \& regulations

Monitoring \& analysis methods

Additional references

Express data 
OHM/TADS from EPA

Oil and Hazandous Materials/Technical Assistance Data System from the Environmental Protection Agency Environmental effects of over 1,200 petroleum products and hazardous materials. OHM/TADS documents include:

Substances

Transport/storage/handling

Laboratory

Physicochemical parameters

Fire/explosion/corrosion hazards

Environmental hazards

Range of toxicity

Human health hazards

Cleanup procedures

Data adequacy evaluation

\section{CHRIS from Coast Guard}

Chemical Hazard Response Information System developed by the U.S. Coast Guard

Release, fire and health hazards of over 1,000 chemicals. CHRIS documents include:

Overview

Response to discharge

Label

Chemical designations

Observable characteristics

Health hazards

Fire hazards

Chemical reactivity

Water pollution

Shipping information

Hazard classifications

Physical \& chemical properties

\section{DOT Emergency Response Guides}

\section{Department of Transportation Emergency Response Guides}

Initial response to fires, explosions and releases involving hazardous chemicals.

Documents include:

Potential hazards

Emergency action

Reference 
IRIS from EPA

Integrated Risk Information System from the Environmental Protection Agency

Information on how 375 chemicals affect human health. It is a primary source of risk

assessment data on chemicals of environmental concern. IRIS documents include:

Introduction

Chronic health hazard assessment for non-carcinogenic effects

Carcinogenicity assessment for lifetime

Health hazard assessments for varied exposure durations

U.S. EPA regulatory actions

Supplementary data

Bibliography

Revision history

\section{SEARCHING THE SYSTEM:}

Chemicals can be retrieved by chemical name or synonym and CAS, NIOSH/RTECS, STCC, UN/NA (DOT), ENT or USAF numbers. After the first entry, searching is accomplished by single keystroke choices from a series of menus. "Backtracking" through the search is also done by single keystrokes. As every screen displays all needed navigation instructions, formal training is not needed and a cumbersome user's manual does not need to be mastered. Several features make searching quick and simple:

HELP SCREENS - Explain instructions for using the system

USING THE SYSTEM - Identifies where specific topics can be found

HOW THE DOCUMENT IS ORGANIZED - Displays subject categories and their subheadings

INTEGRATED INDEX - Offers search capability on specific chemicals in several databases

\section{THE EDITORIAL BOARD:}

The primary responsibility for maintaining quality rests with the TOMES Plus ${ }^{\text {'m }}$ Editorial Board and Editorial Staff, which is comprised of leading professionals in environmental, industrial and reproductive toxicology, occupational medicine and industrial hygiene and safety. The number of member editors continues to rise as the system expands to cover new topics and becomes more comprehensive. Publication of this system has been through the efforts and contributions of the Editorial Board and the following Editorial Staff:

Barry H. Rumack, M.D. Editor

Alan H. Hall, M.D.

Director/Editor

Betty J. Dabney, Ph.D.

Managing Editor
Marta R. Clark, B.S.

Regulatory Analyst/System Coordinator

Pearlanne T. Zelarney, B.S., M.S.

Toxicological Writer/Research Coordinator 


\section{DELIVERY METHODS}

The TOMES Plus Information System is offered via the following delivery methods:

Compact disc for use with personal computers

Computer tapes for IBM and DEC VAX

Other configurations under development.

\section{WHO IS MICROMEDEX?}

In 1974, Micromedex introduced its first product by publishing information on microfiche for Poison Centers in the United States. By 1989, more than 35 countries were relying on the Micromedex databases. In addition to toxicology information, Micromedex now provides drug information, emergency medicine information and other programs. In 1987, the TOMES database was originally derived from the Micromedex POISINDEX ${ }^{\circledR}$ System. In April 1989, the TOMES Plus database was introduced, an enhancement of the original product offering several additional chemical databases. The Micromedex state-of-the-art databases are available on various configurations including compact disc (CD-ROM) and mainframe tapes. A wholly owned subsidiary of Mead Data Central since 1985, Micromedex expects to continue its growth by providing current and comprehensive reference sources. 
Appendix 16

Subject Index 


\title{
Appendix 16 \\ Subject Index
}

\begin{abstract}
A
Accident Investigation, B-3, B-4, K-2

Acids/Bases, E-2, E-11, F-7, F-10

Action Level, I-1, I-3, I-4

Acute Toxins, F-5, G-1, also see Toxic Gases

Administrative Controls - see Controls

ACGIH (American Conference of Governmental Industrial Hygienists), A-2, C-3, F-1, G-7
\end{abstract}

B

Bases - see Acids/Bases

Beryllium, G-19 to G-20

Biohazardous Waste, E-14

Biological Safety Cabinet - see Ventilation

C

Cadmium, G-21 to G-22

Carcinogens, F-5, G-1 to G-14, Appendix 9, 10, \& 11

ACGIH Carcinogens, G-5, G-7, Appendix 10

classification, G-4

clean-up, G-11

controls, G-10 to G-13

disposal, G-12

exposure assessment, G-7, G-9, G-13, G-14, Appendix 12

labeling, G-11

medical surveillance, G-13, J-8

ordering/receiving, D-2, G-8, G-9

OSHA regulated carcinogens, G-6, J-5 to J-7, Appendix 9,

also see Select Carcinogens

personal protective equipment, G-11

Program, G-3, G-7

required considerations, G-2

responsibilties, G-8

safety procedures, G-9, G-11, G-13, Appendix 4

App. 16 - 1 
Select Carcinogens, C-2, G-5 to G-8, G-32 to G-34, Appendix 9 \&11 training, G-9, H-1

Chemicals - see Hazardous Chemicals, see Incompatible

Chemical Infogram, $\mathrm{H}-4$, Appendix 15

Chemical Hygiene Officer, B-4

Chlorinated Hydrocarbons - see Solvents

Chronic Toxins - G-1

Clean-up - see Spills

Clothing - see personal protective equipment (PPE)

Combustibles - see Flammables

Compatible Chemicals - see Incompatible Chemicals

Complaints, B-2

Compressed gases, G-37, also see Toxic Gases, Pyrophoric Gases

Confined Spaces, G-28, G-35, Appendix 14

Construction \& Maintenance Department (C\&M), B-7, F-6, F-15, F-18

Containment - see Controls and Ventilation

Contractor Safety, B-7, B-8

Controlled Substances, D-1

Controls, A-2, C-1, E-1, Section F, G-2 administrative, E-2, F-1, F-11, F-12, also see Designated/Regulated Areas,

criteria for, F-1 to F-4, G-10

Safety Procedures, and Work Practices

engineering, F-4, also see Ventilation

personal - see Personal Protective Equipment (PPE)

Cryogenic Hazards, G-38

\section{D}

Decommissioning, F-18

Decontamination - G-2, G-3, G-8, G-36, also see Spills

Design - see Equipment

Designated Areas - E-2, F-11, G-2, also see Controls, administrative

Disposal - see Hazardous Materials, disposal \& Waste

Drinking - see Eating

\section{E}

Eating, E-3, E-12

EH\&S, B-5, B-6, E-14 to E-16

Emergency Equipment - see Equipment

Emergency Phone Numbers, E-13

Engineering Controls - see Controls 
Equipment

decommissioning, F-18

design, F-5 to F-10, G-13

emergency, F-13 to F-15, E-12, E-13, D-3

preventive maintenance, $\mathrm{B}-7, \mathrm{~F}-16$ to $\mathrm{F}-18, \mathrm{~K}-2, \mathrm{~K}-3$

safety, F-13

eye wash/safety showers, F-14

fire extinguishers, F-15, G-28

flammable storage cabinets, F-15, G-30

gas detector, D-3

guarding, F-7, F-13

personal, see Personal Protective Equipment (PPE)

pressure, F-13

seismic, E-2, E-11, F-7

shielding, F-13

ventilation/exhaust - see Ventilation

Employee, B-1, B-2

EMS (Environmental Management System) Database, Appendix 4

Ethernet MSDS Access, H-4, Appendix 15

Ethers, D-2, G-24, G-25

Explosives - E-11, F-7, also see Reactive Chemicals

Exposure to Chemicals, A-2, F-1, F-2

Exposure Monitoring, Section I

assessment of need, F-2, G-9

criteria for, I-2, I-3

interpretation, I-4

purpose, I-1

records, $\mathrm{K}-1$

reporting/referrals, I-4, I-5

substances requiring, I-3

Eyewash - see Equipment, safety

Facility Notebook, A-4, B-3, G-9, Appendix 4

Fire Department, B-6, C-2, E-6, E-12, F-15, G-16 to G-18, G-23, G-29, G-30, G-36, G-37, K-3

Fire Extinguishers - see Equipment, safety

Fluorocarbons - see Solvents

Flammable Storage Cabinet - see Equipment, safety

Flammables, E-2, F-7, F-8, F-10, F-15, G-26 to G-36

Fume Hoods - see Ventilation, hoods 
G

Gas Detectors-see Equipment, safety

Gas Safety Cabinet - see Ventilation

Gases, G-37, also see Toxic Gases, Pyrophoric Gases

Glassware, E-7

Glossary, Appendix 3

Glove box - see Ventilation

Guarding - see Equipment, safety

Guests - see Visitor Safety

\section{$\mathbf{H}$}

Hazard Assessment, I-2 to I-3, C-2, Appendix 4 \& 12

Hazard Communication Standard (OSHA), A-2, A-3, A-4, Appendix 1

Hazard Control - see Controls

Hazardous Chemicals, C-1 to C-3, Section G, Appendix 9, 10, 11, 12

Hazardous Materials Handling

disposal, E-4 to E-16

inventory, B-3, K-1, Appendix 4

labeling - see Labeling

procurement, B-1, B-5, D-2 Appendix 5

spills - see Spills

storage, E-2, E-3, E-10 to E-12, G-29 to G-31, G-34, Appendix 8

transportation, B-8, E-8, E-15

High Acute Toxicity Chemicals - see Toxins

Hoods - see Ventilation

Housekeeping, E-6, E-16

HVAC (Heating, Ventilation, and Air Conditioning) - see Ventilation, general

Hygiene, E-1, E-2

I

IARC (International Agency for Research on Cancer), G-4, G-5, G-14, Appendix 9 Incompatible Chemicals, E-2, E-14, Appendix 8

Industrial Hygiene Group, B-4, B-5

assistance, D-3, F-7, G-10, G-18, G-34

exposure monitoring, F-1, G-8, G-13, I-1, I-5, J-9

hazard evaluation, B-4, B-5, F-2, G-2, G-9, G-13, G-32, Appendix 12

pre-approval/review, D-2, G-7 
Information

chemicals, H-3, H-4, Appendix 15

references, L-1

\section{$\mathrm{J}-\mathrm{L}$}

Lab Coats, - see Personal Protective Equipment (PPE)

Lab Hoods - see Ventilation

Labeling, E-8, Appendix 7

area, E-8, F-11, G-7, Appendix 7

container, E-1, E-10, E-15, G-34, Appendix 7

equipment, E-12, F-17

refrigerator, $\mathrm{F}-12$

Laboratory Standard - see OSHA

Laboratory Use, A-3

Laminar Flow Hoods, see Ventilation

\section{$\mathbf{M}-\mathbf{N}$}

Maintenance-see Equipment

Materiel Management and Transportation Department, B-8

Medical Services, Section J

exams, J-1

consultations, J-1, J-7

pregnancy and reproduction, J-9

records, $\mathrm{K}-2$

reporting hazards, G-14, I-5, J-4, J-9, K-1

surveillance, J-1 to J-6, G-14

Medical Waste - E-14

Mercury, G-22 to G-24

Metals, G-19

Monitoring - see Exposure Monitoring or Medical Surveillance

MSDS (Material Safety Data Sheet), A-2, B-3, B-6 to B-8, E-1, F-1, G-8, H-2 to H-4, Appendix 15

NTP (National Toxicology Program), G-4, G-5, G-14, Appendix 9

O

OSHA (Occupational Health and Safety Administration), A-2, G-4, Appendix 9 OSHA Laboratory Standard, A-2, A-3, G-1, G-5, G-14, Appendix 2

OSHA Subsiance-Specific Regulations, J-6 
OSP (Operational Safety Procedure), A-5, B-4, C-1, C-4, C-5, D-2, D-3, F-17, F-18 Over Exposure - see Exposure to Chemicals

Oxidizers, E-11, Appendix 8

Particularly Hazardous Substances, G-1 to G-3

PCBs (polychlorinated biphenyls), G-1, G-36

PEL (Permissible Exposure Level), A-2, C-3, F-1, G-1, G-2, G-4, G-33, G-34, I-1, I-3, I-4

Perchloric Acid, F-7

Peroxides, D-2, E-11, G-24

Personal Protective Equipment (PPE), E-4, E-12, F-12, G-11

body and footwear, E-4

clothing, E-4

eye, E-5

gloves, E-4, E-7, G-34 to G-36 Appendix 6

respiratory, E-2,E-5, F-2, G-36

Physical Hazards, C-2, G-37

Pipetting, E-3

Plant Engineering Department, B-7, F-6, G-18

Poisons - see Toxins

Pressure Safety - F-13

Preventive Maintenance - see Equipment

Procurement - see Hazardous Materials Handling, procurement

Proposition 65 (State of California Safe Drinking Water and Toxic Enforcement Act), G-15, Appendix 13

Purchasing Requisition, Appendix 5

Purchasing Department, B-7, G-18

Pyrophoric Gases, D-2 to D-4, F-9, G-16 to G-19

\section{Q-R}

Reactive Chemicals, D-2, E-11, G-24 to G-26

Reactive Peroxides, D-2, E-11, G-24

Recordkeeping, Section $\mathrm{K}$

Regulated Areas - E-2, F-11, G-2, also see Controls, administrative

Reproductive Toxins, G-1, G-14 to G-16, J-9. Appendix 13

Restricted Areas, F-11, also see Controls, administrative

Roles and Responsibilities, B-1 
Safety Equipment - see Equipment

Safety Procedures, B-2, C-1, F-10, G-7, G-9, Appendix 4

Safety Review Committee, B-6

Safety Training - see Training

Safety Training Check List, Appendix 14

Seismic Safety - see Equipment, safety

Select Carcinogen, C-2, G-5 to G-8, G-32 to G-34, Appendix 9 \&11

Sharps - see Glassware, see Wastes

Shields - see Equipment, safety

Showers - see Equipment, safety

Signs - see Labeling

Smoking - see Eating

Solders with cadmium, G-21

Solvents, E-11, G-1, G-7, G-26 to G-36

chlorinated hydrocarbons, G-31 to G-34

flammable/combustible, G-26 to G-31

fluorocarbons, G-35 to G-36

Space, vacating/new occupancy, F-18

Specific Safety Procedure (SSP), A-5, B-2, B-3, C-1, C-4, C-5, D-2, G-7, G-9, Appendix 4 Spills, E-6, E-12

clean-up/decontamination, G-2, G-3, G-8, G-23, G-36

clean-up materials, E-12, E-13, G-36

emergency numbers, E-13

mercury, G-23

Sprinklers - F-7

STEL (Short Term Exposure Limit), F-1, I-4

Storage - see Hazardous Materials Handling

Stores, E-13, Appendix 6 \& 7

TLV (Threshold Limit Value), A-2, C-3, F-1, G-34, I-4

TOMES (Micromedex' Toxicology, Occupational Medicine, \& Environmental Series), H-4, Appendix 15

Toxic Chemicals Subcommittee, B-5, B-6

Toxic Gases, D-2 to D-4, F-9, G-16 to G-19

Toxic Metals, G-19

Toxins, C-2, D-3, G-1 to G-36

high acute, G-1 
Training, A-1, B-2, F-12, Section H, Appendix 4, 14 \& 15 carcinogen, G-9

CHSP Training, $\mathrm{H}-1$

hazard communication, $\mathrm{H}-1, \mathrm{H}-3$

hazardous gas,D-3, G-17

operation-specific training, $\mathrm{H}-1$

records, $\mathrm{K}-2$

required topics, $\mathrm{H}-1$ to $\mathrm{H}-2$

resources, $\mathrm{H}-4$

respirators, E-5

Safety Training Check List, Appendix 14

Transportation - see Hazardous Materials Handling

\section{U-V}

Unattended Operations, E-6

Vacating Lab/Shop Space, F-18

Vacuum With Oxygen, G-38

Variances, C-6

Ventilation, F-4 to F-10, G-28

biological safety cabinets (laminar flow hoods), F-8, F-9

cold room, F-10

environmental rooms, F-9, F-10

exhaust air, F-7, F-8, G-13

gas safety cabinet, F-9

general HVAC, F-4, F-6

glove box, F-8, F-9

hoods, F-3, F-5 to F-8, F-16 to F-17, G-3, G-7

LEV (local exhaust ventilation), F-5, F-6, F-7, F-10

recirculating, F-4, F-5

Violations, B-2

\section{$\mathbf{W}, \mathbf{X}, \mathbf{Y}, \mathbf{Z}$}

Wastes, E-6, E-14, G-34

dry materials, E-15

flammable, E-14, E-15

medical/biohazardous, E-14

oils, E-15

PCBs, G-36

radioactive/mixed, E-14

sharps, E-14, E-16

Work Practices, F-10, H-2, also see Safety Procedures

Working Alone, E-6 

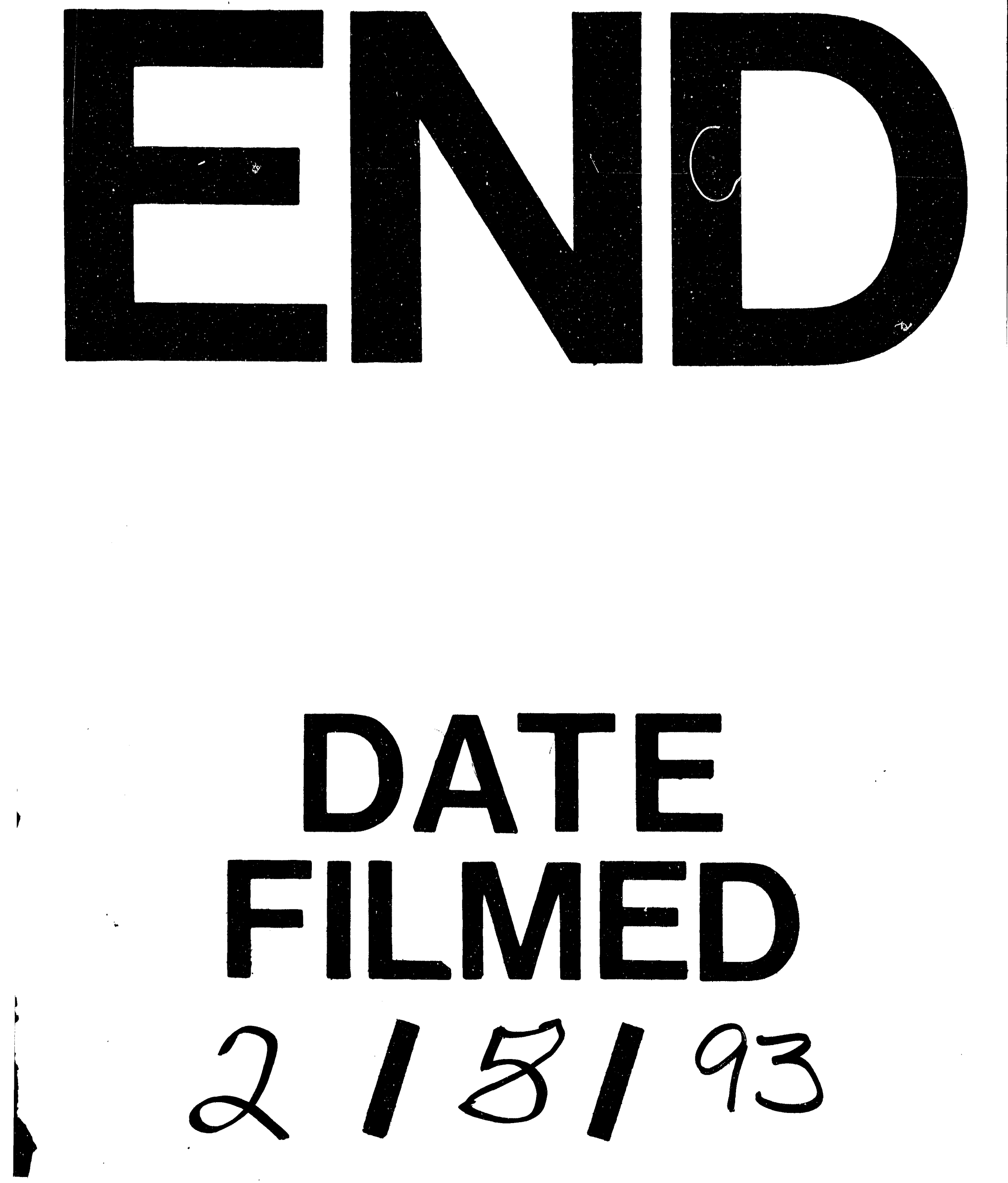